\title{
M Dwarfs in the Local Milky Way: the Field Low-Mass Stellar Luminosity and Mass Functions
}

John J. Bochanski, Jr.

A dissertation submitted in partial fulfillment

of the requirements for the degree of

Doctor of Philosophy

University of Washington

2008

Program Authorized to Offer Degree: Department of Astronomy 



\title{
University of Washington \\ Graduate School
}

This is to certify that I have examined this copy of a doctoral dissertation by

\author{
John J. Bochanski, Jr.
}

and have found that it is complete and satisfactory in all respects, and that any and all revisions required by the final examining committee have been made.

Chair of the Supervisory Committee:

Suzanne L. Hawley

Reading Committee:

Suzanne L. Hawley

Iain Neill Reid

Željko Ivezić

Date: 

In presenting this dissertation in partial fulfillment of the requirements for the doctoral degree at the University of Washington, I agree that the Library shall make its copies freely available for inspection. I further agree that extensive copying of this dissertation is allowable only for scholarly purposes, consistent with "fair use" as prescribed in the U.S. Copyright Law. Requests for copying or reproduction of this dissertation may be referred to Proquest Information and Learning, 300 North Zeeb Road, Ann Arbor, MI 48106-1346, 1-800-521-0600, to whom the author has granted "the right to reproduce and sell (a) copies of the manuscript in microform and/or (b) printed copies of the manuscript made from microform."

Signature

Date 

University of Washington

\title{
Abstract \\ M Dwarfs in the Local Milky Way: the Field Low-Mass Stellar Luminosity and Mass Functions
}

\author{
John J. Bochanski, Jr. \\ Chair of the Supervisory Committee: \\ Professor Suzanne L. Hawley \\ Department of Astronomy
}

Modern sky surveys, such as the Sloan Digital Sky Survey (SDSS) and the TwoMicron All Sky Survey, have revolutionized how Astronomy is done. With millions of photometric and spectroscopic observations, global observational properties can be studied with unprecedented statistical significance. Low-mass stars dominate the local Milky Way, with tens of millions observed by SDSS within a few kpc. Thus, they make ideal tracers of the Galactic potential, and the thin and thick disks. In this thesis dissertation, I present my efforts to characterize the local low-mass stellar population, using a collection of observations from the Sloan Digital Sky Survey (SDSS).

First, low-mass stellar template spectra were constructed from the co-addition of thousands of SDSS spectroscopic observations. These template spectra were used to quantify the observable changes introduced by chromospheric activity and metallicity. Furthermore, the average ugriz colors were measured as a function of spectral type. Next, the local kinematic structure of the Milky Way was quantified, using a special set of SDSS spectroscopic observations. Combining proper motions and radial velocities (measured using the spectral templates), along with distances, the full $U V W$ space motions of over 7000 low-mass stars along one line of sight were computed. These stars were also separated kinematically to investigate other observational differences 

between the thin and thick disks.

Finally, this dissertation details a project designed to measure the luminosity and mass functions of low-mass stars. Using a new technique optimized for large surveys, the field luminosity function (LF) and local stellar density profile are measured simultaneously. The sample size used to estimate the LF is nearly three orders of magnitude larger than any previous study, offering a definitive measurement of this quantity. The observed LF is transformed into a mass function (MF) and compared to previous studies. 



\section{TABLE OF CONTENTS}

Page

List of Figures . . . . . . . . . . . . . . . . . . . .

List of Tables . . . . . . . . . . . . . . . . . . $\quad$ xv

Chapter 1: Introduction . . . . . . . . . . . . . . . 1

1.1 Background: Plates to CCDs . . . . . . . . . . . . 1

1.2 Observational Properties of Low-Mass Dwarfs . . . . . . . . . . . . 3

1.2.1 Spectroscopic Features . . . . . . . . . . . . . . 3

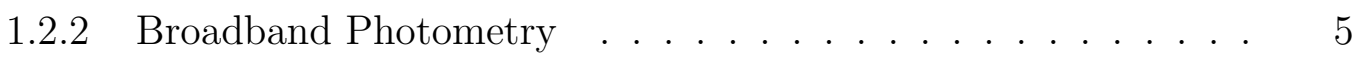

1.3 M Dwarfs \& the Local Milky Way: Low-Mass Stars as Tracers of Galactic Populations . . . . . . . . . . . . . . 6

1.4 Field Luminosity and Mass Functions of Low-Mass Stars . . . . . . . 8

1.5 Thesis Outline. . . . . . . . . . . . . . . . 10

Chapter 2: Low-Mass Dwarf Template Spectra from the Sloan Digital Sky Survey . . . . . . . . . . . . . . . 11

2.1 Chapter Summary . . . . . . . . . . . . . . . 11

2.2 Introduction . . . . . . . . . . . . . . . . . 11

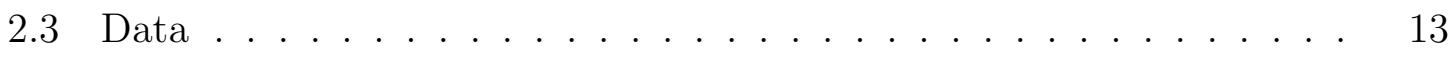

2.3.1 SDSS Photometry . . . . . . . . . . . . 13

2.3.2 SDSS Spectroscopy . . . . . . . . . . . . . . 14

2.4 Analysis . . . . . . . . . . . . . . . . . 15

2.4.1 Spectral Types and Activity . . . . . . . . . . . . 15

2.4.2 Coaddition ...................... 17

2.5 Results and Discussion . . . . . . . . . . . . . . . 22

2.5.1 Radial Velocity Accuracy . . . . . . . . . . . . . . 22

2.5.2 Spectral Differences: Activity \& Metallicity . . . . . . . . 25 
2.5.3 Photometric Differences: Colors . . . . . . . . . . . . . . 33

2.6 Conclusions . . . . . . . . . . . . . . . . . . . . . . . . . . 35

Chapter 3: Exploring the Local Milky Way: M Dwarfs as Tracers of Galactic Populations . . . . . . . . . . . . . . . . 42

3.1 Chapter Summary . . . . . . . . . . . . . . . . . . . . . 42

3.2 Introduction . . . . . . . . . . . . . . . . . . . . . . . . . 42

3.3 Observations . . . . . . . . . . . . . . . . . . . . . 46

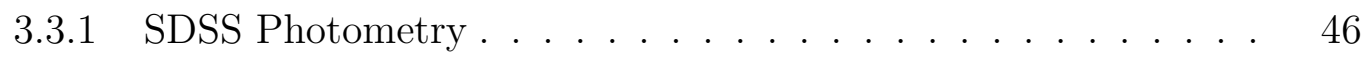

3.3 .2 SDSS Spectroscopy . . . . . . . . . . . . . . . . . 46

3.3.3 SDSS Low-Mass Spectroscopic Sample: SLoMaSS … . . . 47

3.4 Analysis . . . . . . . . . . . . . . . . . . . . . . . . . 48

3.4 .1 Spectral Types . . . . . . . . . . . . . . . 48

3.4.2 Distances - Photometric Parallax . . . . . . . . . . . . . 48

3.4.3 Distances - Spectroscopic Parallax . . . . . . . . . . . . . . . 49

3.4.4 Radial Velocities, Proper Motions \& UVW Velocities . . . . . 49

3.5 Results . . . . . . . . . . . . . . . . . . . 50

3.5 .1 Kinematics . . . . . . . . . . . . . . . . 52

3.5.2 Galactic Dynamical Models . . . . . . . . . . . . . . . 53

3.5.3 Differences between the Thin and Thick Disks . . . . . . . . 60

3.6 Conclusions . . . . . . . . . . . . . . . . . . . . . . . . 68

Chapter 4: Absolute Magnitudes \& Colors of Nearby Low-Mass Dwarfs . . 72

4.1 Introduction . . . . . . . . . . . . . . . . . . . . . 72

4.2 Sample Selection \& Observations . . . . . . . . . . . . . . . 73

4.2.1 Photometric Telescope Observations _. . . . . . . . . 73

4.2 .2 Other Parallax Stars . . . . . . . . . . . . . . . 75

4.2 .3 Contamination . . . . . . . . . . . . . . 76

4.3 Analysis . . . . . . . . . . . . . . . . . . . . . . . . . 82

4.4 Results . . . . . . . . . . . . . . . . . . . . . . . . . . . 82

4.4.1 Color-Absolute Magnitude Relations . . . . . . . . . . . 82

4.4 .2 Comparison to Previous Relations . . . . . . . . . . . . . 84

4.4.3 Absolute Magnitude Transformations . . . . . . . . . . . . 87

4.5 Conclusions . . . . . . . . . . . . . . . . . . . . . . . . 94 
Chapter 5: $\quad$ Luminosity Function of Field Low-Mass Dwarfs . . . . . . . . . 96

5.1 Introduction . . . . . . . . . . . . . . . . . 96

5.2 Observations . . . . . . . . . . . . . . . . . . . . . . . 99

5.2.1 SDSS Photometry . . . . . . . . . . . . . . 99

5.2 .2 Sample Selection . . . . . . . . . . . . . . . . 101

5.3 Analysis: Stellar Density Maps . . . . . . . . . . . . . . . . . . . . 112

5.3.1 Background . . . . . . . . . . . . . . . . . 112

5.3.2 Density Calculation . . . . . . . . . . . . . . . 113

5.3.3 Disk Density Profiles . . . . . . . . . . . . . . . . . 116

5.4 Analysis: Galactic Model Fits and Raw Luminosity Function . . . . . 122

5.5 Analysis: Correcting Systematic Errors . . . . . . . . . . . . . . . . . 129

5.5.1 Systematics: Fitting Algorithm (The "Clean" Catalog) . . . . 131

5.5.2 Systematics: Malmquist Bias . . . . . . . . . . . . . . 131

5.5.3 Systematics: Unresolved Binarity . . . . . . . . . . . . . . 134

5.5.4 Other Systematics . . . . . . . . . . . . . . . . . 138

5.5.5 Other Systematics: Metallicity . . . . . . . . . . . . . . . 142

5.5.6 Other Systematics: Extinction . . . . . . . . . . . . . . . . . 144

5.5.7 Other Systematics: Contamination . . . . . . . . . . . . . 146

5.5.8 Systematic Uncertainties . . . . . . . . . . . . . . . . . . 148

5.6 Results: Galactic Structure . . . . . . . . . . . . . . . . . 149

5.7 Results: Luminosity Function . . . . . . . . . . . . . . . . . . . 152

5.7.1 Comparison to previous results: $M_{r} \ldots \ldots . . . . . . .152$

$5.7 .2 \quad M_{J} \mathrm{LF} \ldots \ldots \ldots \ldots \ldots 154$

5.8 Conclusions . . . . . . . . . . . . . . . . . . . . . . 160

Chapter 6: Mass Function of Field Low-Mass Dwarfs . . . . . . . . . . . . 164

6.1 Introduction . . . . . . . . . . . . . . . . . . . . 164

6.2 Analysis . . . . . . . . . . . . . . . . . . . . . 167

6.2.1 Mass-Luminosity Relations . . . . . . . . . . . . . . 167

6.3 Results . . . . . . . . . . . . . . . . . . . . 170

6.4 Conclusions . . . . . . . . . . . . . . . . . . . . . . . 181

Chapter 7: Conclusions . . . . . . . . . . . . . . . . . 183 


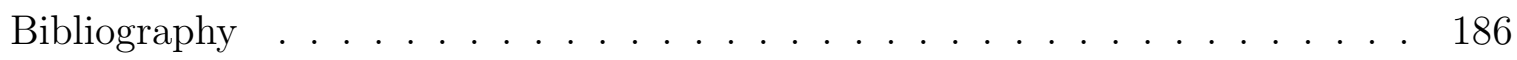




\section{LIST OF FIGURES}

Figure Number $\quad$ Page

2.1 Mean template spectra of active low-mass dwarfs of types M0-L0. The spectral type and number of stars (in parentheses) are labeled for each template. . . . . . . . . . . . . . . . . .

2.2 Mean template spectra of inactive low-mass dwarfs of types M0-M7. No M8-L0 dwarfs met the activity and consistent line velocity criteria. Spectral type and number of stars in each template are labeled. . . .

2.3 Mean composite template spectra for low-mass dwarfs of types M0-L0, formed by combining the data shown in Figures 1 and 2. Spectral type and number of stars in each template are labeled. . . . . . . . . .

2.4 Illustrative template spectra of an inactive M1 star and an active M6 star with strong molecular and atomic features labeled. . . . . . . . .

2.5 Comparison of a high signal-to-noise SDSS M5 spectrum (bottom) to the active M5 template (top) near $\mathrm{H} \alpha$. Note the higher resolution of the template spectrum. . . . . . . . . . . . . . .

2.6 Histogram comparison of high-resolution echelle RV measurements (solid line), medium resolution cross-correlation RV determinations (dashed line), and SDSS pipeline RVs (dotted line) for 19 Hyades M dwarfs. Note the SDSS pipeline reported wildly discrepant velocities for two stars (not shown). . . . . . . . . . . . . . .

2.7 Decrements for the averaged active templates (open diamonds, error bars) are shown. Also shown are results for the dM3e star AD Leo: observed flare (filled circles; from Hawley \& Pettersen, 1991), model flare (open squares), and model quiescent (open triangles) decrements (Allred et al., 2006). Flaring atmospheres have increased density at chromospheric temperatures, resulting in higher opacity and increased emission in the higher-order Balmer lines compared to $\mathrm{H} \alpha$. The result is a relatively flatter decrement. . . . . . . . . . . . . . . 
$2.8 \mathrm{TiO} 2$ vs. TiO4 for active (open triangles) and inactive (filled circles) templates. At a constant $\mathrm{TiO} 4$ value, $\mathrm{TiO} 2$ is deeper (smaller index values) in the active stars, confirming the result originally discussed in Hawley et al. (1996). . . . . . . . . . . . . . . . . . . .

2.9 Shown is an illustrative example of our flux-ratio analysis. The active M0 spectral template (top) is divided by the inactive (middle) template. The resulting flux ratio is plotted on the bottom. The windows display the approximate wavelength bounds of the SDSS $g, r$, and $i$ filters. 32

2.10 The ratio of the active to inactive flux for the M4-M7 templates is shown in the vicinity of the $\mathrm{CaOH}(6230 \AA)$ bandhead. Note the increase in the ratio at later types, indicating a shallower $\mathrm{CaOH}$ band in the active template compared to its inactive counterpart. Apparently, the formation mechanism of $\mathrm{CaOH}$ depends on both the star's spectral type (effective temperature) and the presence of a chromosphere. . . .

2.11 Shown are illustrative examples of our flux ratio analysis of an M0 subdwarf (left panel) and an M1 Hyades star (right panel). The subdwarf and Hyades star are the top spectra in their respective panels; the composite template of the same spectral type (from Figure 2.3) appears as the middle spectrum, and the ratio of the two spectra is shown on the bottom. . . . . . . . . . . . . . .

3.1 Upper panel: Distribution of spectral types within SLoMaSS. The mean $i-z$ color at each spectral type is shown above each bin. Lower panels: $i$ vs. $i-z$ color-magnitude distribution and $r-i$ vs. $i-z$ color-color diagram of stars in SLoMaSS. The lowest contour (black) in the color-color diagram indicates a density of 100 stars in a square 0.1 magnitudes wide. Each additional contour represents 100 additional stars. . . . . . . . . . . . . . . . . . . .

3.2 The absolute vertical distance distribution of the sample binned every 100 pc. The solid thick histogram is the total distribution, while the five remaining histograms represent the height distribution for five $i-z$ color bins as described in the legend. Note that there are hundreds of stars per bin out to an absolute vertical distance of $\sim 1000$ pc. . . . . 
3.3 Schematics of velocity distributions (left panels) and their corresponding probability plots (right panels). The upper row displays two Gaussian distributions, one with a standard deviation of $\sim 50 \mathrm{~km} \mathrm{~s}^{-1}$ (solid line) and another with a standard deviation of $\sim 100 \mathrm{~km} \mathrm{~s}^{-1}$ (dashed line). A straight line in the probability plot signifies an underlying Gaussian distribution and the slope of the line is a measure of the standard deviation of the distribution. Note that the slope of the dashed line is twice that of the solid line. In the bottom row, a low dispersion component (dashed line) and high dispersion component (dotted line) are summed to produce the solid histogram. While the dual nature of the distribution is not readily apparent in the solid histogram, it is easily detected in the probability plot. . . . . . . . . . . .

3.4 Illustrative example of our velocity dispersion analysis. Shown are probability plots for $U, V$ and $W$ for the $1200<z<1300$ pc bin (solid lines). The "core" component (dashed line) is fit between $\pm 1 \sigma$, while the "wing" component (dot-dashed line) fits the outer edges of the distribution. Note the strong asymmetric drift component manifested as a change in slope at negative $V$ velocities. . . . . . . . . . . .

3.5 Velocity Dispersion as a function of vertical distance from the plane in 100 pc bins. The open squares represent the kinematically colder, core component and the crosses represent the high-dispersion, wing component. Errors are derived from the linear fits to the probability plots. Note the smooth trend in the low-dispersion component as a function of height from the Plane. . . . . . . . . . . . . . . .

3.6 Shown are the $R-I$ and distance distributions of the stars in SLoMaSS (solid line) and a sampled model (dashed line). Note the agreement between the two datasets, indicating that we are sampling each instance of the Besançon model in a manner consistent with the spectroscopic observations in SLoMaSS. . . . . . . . . . . . . . . .

3.7 Probability Plots of the $U, V$ and $W$ velocites for pure thin disk (shaded line) and thin + thick models (solid line) in the $1200<z<1300$ pc bin. This height bin corresponds to the SLoMaSS data shown in Figure 3.4. Note that when the thick disk is added to the distribution, the overall slope increases, and the wing components are enhanced, as seen in the $W$ probability plot. The combined thin and thick disk velocity distributions are also necessary to match the two-component structure seen in the SLoMaSS probability plots. . . . . . . . . . . . . 
3.8 Thin Disk (upper panels) and Thick Disk (lower panels) velocity dispersions for SLoMaSS data (open squares) and Besançon model predictions (crosses). The model reproduces general trends, such as increased dispersion with distance from the Galactic Plane. The thin disk $W$ velocity dispersions are systematically low, as are the thick disk $V$ dispersions at large heights. This is probably due to the assumed age-velocity

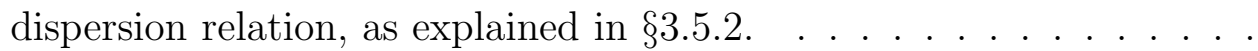

3.9 Thin Disk (upper panels) and Thick Disk (lower panels) mean velocities for SLoMaSS data (open squares) and Besançon model predictions (crosses). The thin disk mean $V$ velocities predicted by the Besançon models are systematically low, while the observed thick disk mean $V$ velocities diverge at large Galactic heights. . . . . . . . . . . . . .

3.10 The metallicity-sensitive ratio of $(\mathrm{CaH} 2+\mathrm{CaH} 3) / \mathrm{TiO} 5$ vs. Spectral Type for the thin (open squares) and thick (crosses) disk populations. Higher ratio values indicate a higher metallicity (Burgasser \& Kirkpatrick, 2006). Within the error bars, the thin and thick disk populations exhibit very similar behavior, indicating that the sample may not be probing a large spread $(\gtrsim 1$ dex) in metallicity. . . . . . . . .

3.11 The ugriz colors as a function of spectral type for the thin (open squares) and thick (crosses) disk populations. The colors of both populations agree within the errors, suggesting that the M dwarfs in SLoMaSS do not cover a large metallicity range. . . . . . . . . . . . .

3.12 Shown is active fraction of stars as a function of spectral type for the thin (open squares) and thick (crosses) disk populations. An older population would show a lower active fraction, as suggested by the thick disk stars in the M5 bin. . . . . . . . . . . .

4.1 $M_{r}$ vs. $r-i$ CMD. The parallax stars listed in Table 4.1 are shown as filled circles and the best fit line from Table 4.2 is the solid red line. Other existing parallax relations are plotted for comparison: Hawley et al. (2002) (dark dash-dot line), West et al. (2005) (purple dashdot line), Jurić et al. (2008) (green dash-dot line), Sesar et al. (2008) (yellow dash-dot line), Golimowski et al. (2009) (solid blue line). The original Hawley et al. (2002) and West et al. (2005) relations have been transformed using data from their color/absolute-magnitude tables. In addition, the 5 Gyr isochrone from the Baraffe et al. (1998) models appears as the dashed line. The differences between the CMR from this study and the others on this plot are shown in Figure 4.5. . . . . 
4.2 Color-color diagrams of the SDSS photometric sample (contours) with the trig parallax stars overplotted as filled red circles. The contours represent $0.2 \%$ of the entire sample, with contours increasing every 10 stars per 0.05 color-color bin. The typical color error bars of the parallax sample stars are smaller than the plot symbols. These diagrams demonstrate that the nearby star sample is drawn from the densest regions of the low-mass stellar locus and extend beyond the SDSS color-color space. . . . . . . . . . . . . . . .

4.3 $M_{r}$ vs. $r-z$ CMD. The parallax stars listed in Table 4.1 are shown as filled circles and the best fit line from Table 4.2 is the red solid line. The best fit from Golimowski et al. (2009) is shown as the blue line and the CMD from West et al. (2005) is plotted as the purple dash-dot line. The CMR of Hawley et al. (2002) is shown as the dark dash-dot line. The 5 Gyr isochrone from the Baraffe et al. (1998) models is shown as the dashed line.

4.4 $M_{r}$ vs. $i-z$ CMD. Same symbol definitions as Figure 4.3. Note the very poor agreement between the observations and model predictions.

4.5 Differences between the $M_{r}$ vs. $r-i$ relations in Figure 4.1. The line styles are the same as Figure 4.1. Note that the majority of other photometric parallax relations agree at $\sim 0.2$ mags, while the $5 \mathrm{Gyr}$ isochrone of Baraffe et al. (1998) fails to agree with the empirical results.

4.6 Differences between the $M_{r}$ vs. $r-z$ relations in Figure 4.3. Line styles are the same as Figure 4.3. As in Figure 4.5, there is excellent agreement between the Golimowski et al. (2009) CMR and our own. .

4.7 Differences between the $M_{r}$ vs. $i-z$ relations in Figure 4.4. Line styles are the same as Figure $4.4 . \ldots \ldots \ldots$. . . . . . . . .

4.8 $M_{J}$ magnitude vs. $M_{r}$ magnitude. Shown in red is a $5^{\text {th }}$ order bestfit polynomial, allowing the transformation from $r$ band to $J$ band absolute magnitudes. The dashed blue line is the relation constructed from a $\left(M_{r}, g-i\right) \mathrm{CMR}$ and the analytic fits from Covey et al. (2007b). The rms scatter about the fit is $\sim 0.16 . \ldots \ldots$. . . . . .

4.9 Fractional distance errors $\left(\sigma_{d} / d\right)$ as a function of $r-z$ color and $r$ magnitude. Notice for most of the sample, our distance errors are better than 20\%. This is a direct result of the accurate photometry from SDSS and the moderate slope of our adopted $\left(M_{r}, r-z\right)$ relation. Overplotted are lines of constant distance. . . . . . . . . . . . . 
5.1 The Aitoff projections of the SDSS DR6 Legacy footprint in equatorial (top panel) and Galactic (bottom panel) coordinates. To aid figure clarity, only $0.2 \%$ of the final sample is shown. . . . . . . . . . .

5.2 Histograms in $r, i$ and $z$ showing the effects of flag cuts on the sample. Each flag is labeled with a different color as noted in the legend. The "clean" sample (dashed line) is complete from $16<r<22$. . . . . .

5.3 Shown in the panels are the $r-i, i-z$ color-color diagrams for the various flag cuts discussed above. The contours increase at $10 \%$ intervals. Of note are the relatively wide loci of the objects with the PSF_FLUX_INTERP and INTERP_CENTER flags set. BAD_COUNTS_ERROR objects also demonstrate considerable scatter. SATURATED objects are mostly bluer, indicating that they are probably higher luminosity. 107

5.4 Histograms of brightness for the final stellar sample in $r, i$, and $z . \quad$. . 109

5.5 Histograms of color for the final stellar sample in $r-i, i-z$, and $r-z .110$

5.6 Color-color diagrams of the final photometric sample with the $5 \mathrm{Gyr}$ isochrones of (Baraffe et al., 1998) (red dashed line) and (Girardi et al., 2004) (yellow dashed line) overplotted. The contours represent $0.2 \%$ of our entire sample, with contours increasing every 10 stars per 0.05 color-color bin. Note that the model predictions fail by more than a magnitude in some locations of the stellar locus.

5.7 Star counts as a function of Galactic $R$ and $Z$ for a 0.5 magnitude slice in absolute magnitude centered on $M_{\mathrm{r}}=9.75$. The color bar on the lower part of the plot displays the scale of the image. The number of stars in this absolute magnitude slice is at the top of the plot. The majority of the stars in the sample are found in the northern Galactic hemisphere, since SDSS was centered on the Northern Galactic Cap.

5.8 Volume probed by SDSS imaging as a function of Galactic $R$ and $Z$ for one $0.5 \mathrm{mag}$ slice at $M_{\mathrm{r}}=9.75$. The corresponding scale (in $\mathrm{pc}^{3}$ ) is at the bottom of the plot.

5.9 Density (in stars $\mathrm{pc}^{-3}$ ) as a function of Galactic $R$ and $Z$. The logarithmic scale is shown beneath the density map. The disk structure of the Milky Way is clearly evident, with a smooth decline towards larger $R$, and an increase in density approaching the Plane $(Z=0) . \quad$. . .

5.10 The fractional error in density as a function of $R$ and $Z$. As in the previous figures, the scale is below the map. The errors, calculated in Equation 5.6 are $\lesssim 7 \%$ for the majority of the sample. . . . . . . . 
5.11 Stellar Density Maps from $M_{r}=6.75$ to $M_{r}=8.25$. Note the smooth exponential disk structure in each (0.5 mag) absolute magnitude slice.

5.12 Shown is the "raw" $r$-band LF for the stellar sample, using the $\left(M_{r}, r-\right.$ z) CMR. Note the smooth behavior, with a peak near $M_{r} \sim 11$, corresponding to a spectral type of $\sim$ M4. The error bars are the formal uncertainties from fitting the local densities in each $0.5 \mathrm{mag}$ absolute magnitude slice in stellar density. . . . . . . . . . . . . . .

5.13 Shown are two $(r, i-z)$ Hess diagrams from the SDSS photometry, before (top panel) and after (middle panel) the flag cuts described above. Each contour represents $10 \%$ of the stars. The ratio of the two diagrams (bottom panel) is used to statistically enforce the behavior of SDSS flag and brightness cuts on the Monte Carlo catalogs. Each contour in the bottom panel is labelled, and for most locations in the Hess diagram, the flag cuts preserve $<80 \%$ of the stars. . . . . . . .

5.14 Shown are the derived luminosity functions for the Malmquist bias Monte Carlo model with (red line) and without (blue line) binaries included. The largest effect is seen at large luminosities, where the number of stars observed is larger. At the faint end, the density of binaries is over-estimated, since many binary systems at these luminosities are composed of equal mass stars. This artificially shrinks their distances, increasing the local density. . . . . . . . . . .

5.15 Shown are the LFs measured from density maps computed with the $\left(M_{r}, r-z\right)$ (filled circles) and $\left(M_{r}, r-i\right)$ (open squares) CMRs. Their broad agreement in shape confirms that the CMRs used in this analysis are consistent. The differences from $M_{r} \sim 10-12$ are representative of the true uncertainty in the LF. . . . . . . . . . . . . . . . .

5.16 Shown are the systematic changes in the LF induced by shifting the $\left(M_{r}, r-z\right)$ CMR by +0.5 (red line) and -0.5 (blue line) mags in $M_{r}$, together with the raw LF. The dashed histograms correspond to a thin disk fraction held fixed at 0.96. Note that the shift in absolute magnitude moves the peak of the LF to higher or lower luminosities, corresponding to the sign of the shift. Furthermore, holding the normalization fixed does not induce large relative changes in the resulting LFs, as the solid and dashed histograms are virtually indistinguishable. 141 
5.17 Shown are the differences in the LF induced by metallicity gradients, along with the raw LF (black line). The red histogram corresponds to the extreme limit, where all stars are metal-poor $([\mathrm{Fe} / \mathrm{H}] \sim-0.65)$. The yellow histogram shows the effect of the metallicity gradient from Equation 5.14, without fixing the thin disk / thick disk normalization. The blue histogram shows the measured LF with a metallicity dependent CMR and a fixed thin disk fraction $(f=0.96) . . . . . . .$.

5.18 Shown is the systematic effect of extinction on the raw LF. When no extinction correction is applied (open squares), distant stars act to inflate the local densities of the brightest stars, compared to the fiducial case (filled circles). At fainter luminosities, this effect becomes less important. . . . . . . . . . . . . . . . . . .

5.19 Shown is the raw LF (black line) along with the corrected LFs. The correction for Malmquist bias (blue line) and Malmquist bias with binaries (red line) is quite similar at high luminosities (indicating binaries do not strongly influence the LF), but at low luminosities, the binary correction becomes more important. . . . . . . . . . . . . . .

5.20 Shown is the average, bias-corrected LF, along with individual LFs measured with five different CMRs. The colors corresponding to each CMR are given in the legend. Since the CMR is the dominant systematic in this analysis, error bars were chosen to reflect the entire observed spread in each LF bin. . . . . . . . . . . . . . . . . . . . .

5.21 Shown is the $M_{r}$ LF (filled circles) compared to the LF measured by Hawkins \& Bessell (1988) (open squares). Note the agreement in the location of the peak of each LF. . . . . . . . . . . . . . .

5.22 Shown is the $M_{r}$ LF (filled circles) compared to the LF measured by Jurić et al. (2008) (open squares). Their sample did not probe fainter absolute magnitudes, and employed a different CMR, which accounts for the offsets between the LFs. . . . . . . . . . . . . . . .

5.23 Shown is the $J$ band LF for this sample. Note the peak near $M_{J}=8$ and the monotonic decline at lower magnitudes. . . . . . . . . . .

5.24 Shown is the $M_{J}$ LF compared to the LF measured by Covey et al. (2008). Note the excellent agreement in shape and scaling, ensuring that the densities estimated by this study are correct. . . . . . . . . .

5.25 Shown is the $M_{J}$ LF (filled circles) compared to the LF measured by (Reid et al., 2002) (open squares). The two LFs agree, resolving previous discrepancies between the photometric and volume-complete samples. . . . . . . . . . . . . . . . . 
6.1 Shown are the empirical mass-luminosity relations (MLRs) of Henry \& McCarthy (1993); Kroupa \& Tout (1997); Delfosse et al. (2000) and Xia et al. (2008) along with M dwarfs of known mass from Reid \& Hawley (2005) and the theoretical predictions of Baraffe et al. (1998). The color of each line is given in the legend. Note that in general, the observational and theoretical predictions agree quite well. . . . . . .

6.2 Shown is the best fit $J$ band LF from Figure 5.23, along with the derivative of the mass- $M_{J}$ relations of Kroupa \& Tout (1997) (solid yellow line) and Baraffe et al. (1998) (dashed line). Note that the peak in the LF corresponds to a maximum in $d \mathrm{M} / d M_{J}$, indicating that a broad range in mass is being mapped into a small range in absolute

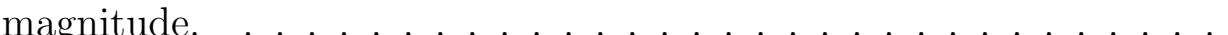

6.3 Shown is the mass function (filled circles) found by transforming the best fit $M_{J}$ LF from Figure 5.23 with the mass- $M_{J}$ relation of Delfosse et al. (2000). The best fit power law (dashed line) and log-normal (dash-dot line) are listed in Table 6.3. . . . . . . . . . . . . .

6.4 Shown is the mass function (filled circles) along with the best fit broken power law (dashed line). The power law break occurs at $\log \mathrm{M}=-0.5$, and provides a better fit than the single power law. The $\alpha$ for each power law segment is quoted in Table 6.3. . . . . . . . . . . . . .

6.5 Shown is the MF from this dissertation (filled circles) and the MF from Covey et al. (2008) (open squares). My best log-normal fit is overplotted (solid line) and compared to the fit from Covey et al. (2008) (dashed line). Both the data and fits are in agreement; the largest differences lie within the uncertainties. . . . . . . . . . . . . .

6.6 Shown is the MF from this dissertation (filled circles) and the MF from Reid \& Gizis (1997) (open squares). Instead of a log-normal fit, a power-law is fit and overplotted (solid line) and compared to the powerlaw fit from Reid \& Gizis (1997) (dashed line). Again, the data are in excellent agreement, with only the lowest mass bin falling outside the formal 1- $\sigma$ error bars.

6.7 Shown is the MF from this dissertation (filled circles) and the MF from Zheng et al. (2001) (open squares). Power law fits are shown for this study (solid line) and the Zheng et al. (2001) results (dashed line). Again, the actual data agree well, despite the discrepancy between the power law fits. 
6.8 Shown is the MF data and best log-normal fit from this study (solid circles and red line), along with the analytic MF fits of Chabrier (2003a) (green dashed line), Kroupa (2002) (dark blue dash-dots line) and Miller \& Scalo (1979) (light blue dash-dot line). The Chabrier (2003a) fit is the closest to the present study, but I stress that comparing actual MF data is more valid than comparing analytic fits. . . . . . . . . 177

6.9 Shown is the MF and best log normal fit from this study (red filled circles and solid line), the Reid \& Gizis (1997) MF (open squares) and the Pleiades MF (green triangles, Moraux et al., 2004). The best fit from this study is extended to higher masses and systematically underpredicts the density at high mass. I stress that analytic fits should only be applied over their quoted mass ranges. . . . . . . . . . . . . 179 


\section{LIST OF TABLES}

Table Number $\quad$ Page

2.1 DAS Query Color Ranges . . . . . . . . . . . . . . 37

2.2 Active Template Decrements . . . . . . . . . . . . . . . . 38

2.3 Active Template Equivalent Widths and $L_{\mathrm{H} \alpha} / L_{\mathrm{bol}, i-z} \ldots \ldots \ldots$

2.4 Template Bandheads . . . . . . . . . . . . . . . . . . . 39

2.5 Template Bandheads . . . . . . . . . . . . . . . . . . 40

2.6 Template Colors . . . . . . . . . . . . . . . . . . 41

3.1 Previous Low-Mass Stellar Kinematic Survey Results . . . . . . . . . 71

4.1 ugrizJHK Photometry of Trig. Parallax Stars . . . . . . . . . . . . . 80

4.1 ugrizJHK Photometry of Trig. Parallax Stars . . . . . . . . . . . . . 81

4.1 ugrizJHK Photometry of Trig. Parallax Stars . . . . . . . . . . . . . 83

4.2 Color- Absolute Magnitude Relations in the ugriz system . . . . . . 84

5.1 Modern Major Low-Mass Stellar Field LF Studies . . . . . . . . . . . 100

5.2 Flag Cuts in SDSS sample . . . . . . . . . . . . . . . . 108

5.3 Measured Galactic Structure . . . . . . . . . . . . . . . . . . 130

5.4 Monte Carlo Galactic Structure Results . . . . . . . . . . . . . . . . . 136

5.5 Best Fit Galactic Structure . . . . . . . . . . . . . . . . . . . . 136

5.6 Effects of Systematic CMR changes on Galactic Structure Results . . 151

5.7 Galactic Structure Parameters . . . . . . . . . . . . . . . . . . 152

5.8 Final $M_{r}$ Luminosity Function . . . . . . . . . . . . . . . . . . . 153

$5.9 M_{J}$ Luminosity Function . . . . . . . . . . . . . . . . . . . 158

6.1 Major Low-Mass Field IMF Studies . . . . . . . . . . . . . . . . . 168

6.2 Mass Function . . . . . . . . . . . . . . . . . . . 178

6.3 Mass Function Analytic Fits . . . . . . . . . . . . . . . . . . . . . 180

6.4 Local Mass Density Estimates . . . . . . . . . . . . . . . . . . . 181 


\section{ACKNOWLEDGMENTS}

Not to sound clichéd, but this dissertation would not have been possible without the support and guidance of many people that I have had the privilege to know and work with over the last few years. They have enriched my life in countless ways, and for that, I owe them all a deep debt of gratitude.

To the teachers and mentors that I have had throughout my academic training, I thank all of you for engaging my mind and imagination, and sharing your time and wisdom. My advisor, Suzanne Hawley, has been a friend and role model. She has set an amazing example for me to aspire to, and I know that the lessons she has taught me over the last six years have prepared me well for the future. Despite her best efforts, there still may be some "flowery" language sprinkled throughout this thesis ${ }^{1}$. I'll miss chatting with her nearly every day, even when she was juggling multiple phone-cons and meetings. And of course, I thank her for inviting me to Dabob Bay, and getting a chance for some R\&R. Ed Guinan, my undergraduate advisor at Villanova, has remained an important source of wisdom and knowledge in my life. I will always appreciate the chance that Dr. G gave me as a freshman, working on a brown dwarf orbiting V471 Tauri. This first taste of research has developed into a passion, and I thank him for opening that door (and hosting my first colloquium)! I'd like to thank Neill Reid for hosting my visit to Space Telescope and serving on my committee. His knowledge and expertise has enriched this work, and I hope a fraction of his academic prolificity rubs off on me. I'd like to thank Željko Ivezić for showing me the beauty of the SDSS way, answering my endless questions on the nuances of

\footnotetext{
${ }^{1}$ Hopefully relegated to the footnotes!
} 
flags and photometry. His dedication to academia is to be commended, taking an early draft of this document to bed, apparently it was that hard to put down.

Thanks to the other members of my committee: Paula Szkody and Scott Anderson. I appreciate your input and advice on this dissertation, which no doubt has improved this work. Special thanks to Oren Etzioni, my GSR, for helping me out at the last minute, and calling me out on all those moving screens. Terry Oswalt, my SARA REU advisor showed the fun of observing'. Brian Abbot, another 'Nova alum, helped with the visualization of this dataset, showing that theorists aren't the only astronomers with cool movies. Julie Lutz has offered me the opportunity for public outreach, and I thank her for the opportunity to share SDSS with the public. Eric Agol has been nothing but helpful, fielding questions on planets and binaries, and offering me new chances in new collaborations.

The other faculty members of the Washington Astronomy Department have a special place in my heart, particularly those that taught my classes: Henny Lamers, Julianne Dalcanton, Tom Quinn, Paul Boynton and Bruce Balick. I will always cherish my chats with George Wallerstein, covering everything from Korea to Hank Aaron and back again, but always talking baseball. I will miss those talks. Ivan King has greatly improved my presentations, and I thank him for his advice along the way.

Richard Coffey, an IT guy with saintly patience, deserves special recognition. He timely addressed all my computing concerns, and introduced me to the beauty of check-pointing. I thank him for putting up with my slow code and bulky datasets. The amazing front office staff: Pat Taylor, Sarah Garner and Stan Vlcek are always quick with a smile and a story, and I thank you for prompt responses to all my questions and forms. Lorenzo and Ronnie deserve special thanks, keeping the department in top shape and tolerating the foosball room.

\footnotetext{
2 and trips to Nogales!
} 
My friends among the Astronomy grad students have made my time in Seattle special. Andrew West has been a great friend and role model. I thank him for answering my endless questions and repeating or rephrasing his explanations. I am looking forward to working with him again soon. Kevin Covey, perhaps one of the nicest people I know, has been a wealth of knowledge and compassion. Our weekly chats are a pleasure, and without him, none of this work would be possible. Marcel Agüeros, despite being a Cowboys and Yankees $\operatorname{fan}^{3}$, has set an example that I strive to follow. Andrew Becker has been a close friend, always taking the time to listen to my rants and lend a compassionate ear when needed. I'll miss hanging out at the IMA and Monday Night Football. Chris Laws, along with the entire Laws clan, has always welcomed me with open arms. His leadership and passion, both in the classroom and on the softball field, is something I will never forget. Sean Raymond and Rory Barnes eased my cross-country transition, and showed me the ropes of the department. My past office-mates: Oliver Fraser and Joel Allred, made coming into work a pleasure, and I hope some of their ingenuity, either with making blinds or hacking Linux, rubs off. To my current office-mates: Nick Cowan and Eric Hilton, I hope you don't fight over the window seat. I'll miss our baseball games, office pranks and the Christmas cards. To my classmates: Lucianne Walkowicz, Alyson Brooks, Mark Claire and Mike Solontoi, you all have a special place in my heart. We plowed through that qual, and came out the other side. I wish you all the best in the future. Special thanks goes to Lucianne, for tolerating my music over the last six years, and letting me steal her copy of NLDS2 for months on end. Daryl Haggard and Rok Roskar are kind souls with refined musical tastes and I'll miss hanging out at their BBQs. The newer grad students: Sarah Schmidt, Adam Kowalski, Sarah Loebman, and Chelsea MacLeod, you are the people who make the department special. Good luck with the rest of

\footnotetext{
${ }^{3} \mathrm{I}$ am still not sure how that works.
} 
your time here, and thanks for making my final years special. Rich Plotkin is one of the funniest people I have ever met, and I will miss chatting about baseball for endless hours. You deserve a t-shirt with your face on it. I've found a great friend in Nate Kaib. I'll always remember the 26.2 miles up in Vancouver, and the countless training runs. Just let me know when you're ready for the next one, and I'll be there. Apologies to Nate and Nick, for being running's angel of death. Also, apologies and thanks to Peter Yoachim, for not destroying me after accidentally over-writing his thesis.

During my time at UW, I've been part of a smattering of quasi-athletic teams. First off, the CS Friday morning basketball crew: Jon Ko, Seth Bridges, Jon Carlson, Travis Kiplean, Ian Lasas and Jon Froelich made waking up and playing at nine in the morning something to look forward to. Of course, the Infrared Sox will always be a part of me. I've enjoyed playing on our championship winning team(s), and can't wait to give it one more crack in the summer. Chris Woods, horseplayer/shortshop extraordinaire gets special thanks for finally helping the team over the hump. Finally, the foosers, young and old, deserve special recognition for the countless hours of competition and providing a much-needed distraction from work.

My friends outside the Astronomy department have really made Seattle a special place to me. Matt Sullivan, armed with one of the quickest minds and sharpest wits I've ever come across, is a true friend. I'll miss all the football games and cookouts. Claire Connell is a kind Irish soul, and I'd like to thank her for listening to all my stories over the years. Karen Rutherford, Canadian, is one of the lucky ones. I wish her the best with her move to Philly, and will always pull for the Canadiens (if they aren't playing the Flyers).

To the friends back home that have supported me over the past six years, I thank you. Dr. Chris Appleman, who knew that after 10 years we'd still be such great 
friends. Good luck with the move to Portland, and I'll be sure to visit you for a change. I still can't believe you beat me to it. Greg Sayegh, you'll be in the club soon $^{4}$. Thanks for the countless hours over the phone, and really being a brother from another mother. Thanks to Courtney Abbott for putting me up in New York over the years, and I am excited to be headed back to the East Coast, since she knows that's where I belong. Jamie O'Neill, one of the kindest people I know. Good luck at Notre Dame and I'll never forget hanging out in Georgetown, or Pat Burrell.

Finally, I'd like to thank my family. My siblings: Dan, Michelle and Bill truly are my best friends and I thank them for their constant love and support. Dan, I wish you and Julie the absolute best, and can't wait to celebrate at the end of this hectic summer. Michelle, you're welcome up in Boston anytime, and I'll probably make a few visits down to the International Village. Slick, good luck with the rest of your time at Villanova, and keep out of (duuble) trouble. To my parents: John and Ginny, your love and support over these past six years have been incredible. It has made the distance seem small, and has put my heart at ease through the tough times. Without your strength and leadership, this dissertation never happens. Thank you for all of your support over the years, and for the understanding when I couldn't make it back to Jersey.

\footnotetext{
${ }^{4}$ Only a year to go!
} 


\section{DEDICATION}

To my parents: John and Ginny Bochanski. Your love and encouragement has carried me along, and the example that you've set has made me work harder when the going got tough. Thank you. 



\section{Chapter 1}

\section{INTRODUCTION}

\subsection{Background: Plates to CCDs}

Low-mass dwarfs $\left(\mathrm{M}<0.8 \mathrm{M}_{\odot}\right)$ are the smallest, coolest members of the Main Sequence. Of all the stars visible to the naked eye, only one is an M dwarf ${ }^{1}$ (AX Mic, $V \sim 6.7)$. Yet, these diminutive stars constitute nearly half of the stellar mass of the Milky Way, and about $80 \%$ of all stars by number. They are the main product of star formation and play an important role in the structure and evolution of the Galaxy. However, due to their low luminosities $\left(L \lesssim 0.01 L_{\odot}\right)$, observations of $\mathrm{M}$ dwarfs are challenging, especially at large distances.

Prior to the 1990s, most photometric surveys of low-mass dwarfs were limited by one of two factors: depth or areal coverage. Observations that probed large solid angles were restricted to relatively bright magnitude cutoffs, surveying the local solar neighborhood to about a hundred parsecs. Alternatively, deep, pencil-beam surveys extended observations to very faint magnitudes, but were often restricted to solid angles of less than a square degree. These factors diminished the number of lowmass stars observed, with the largest samples comprising a few thousand $\mathrm{M}$ dwarfs. Proper motion studies, particularly those of Luyten (1979) and Giclas et al. (1971), contributed the largest number of stars to the low-mass stellar census. Spectroscopic samples of M dwarfs were also small, with the largest studies composed of a few hundred stars (e.g. Vyssotsky, 1956). However, despite these "limitations" a plethora of scientific discoveries came from the study of these tiny, dim stars. Most notable are extensive luminosity function investigations, which began with Dutch astronomers

\footnotetext{
${ }^{1}$ Throughout this thesis, I will use low-mass dwarfs and M dwarfs interchangeably.
} 
(e.g., Kapteyn, 1902, 1914) around the start of the 20th century and continues to the present day (e.g., Reid et al., 1999, 2002; Cruz et al., 2007).

During the 1990s, observational astronomy underwent a paradigm shift. CCD detectors became affordable and deep photometric surveys were carried out over wide areas on the sky. These projects, such as the Sloan Digital Sky Survey (SDSS, York et al., 2000) and the Two-Micron All Sky Survey (2MASS, Skrutskie et al., 2006), can trace their roots back to photographic surveys, epitomized by the National Geographic Society - Palomar Observatory Sky Survey (POSS-I, Minkowski \& Abell, 1963) and its successor, POSS-II (Reid et al., 1991). While the POSS studies used photographic plates and required measuring machines to estimate brightnesses, modern surveys employ CCD photometry to determine the brightness of an object to high-precision (typically $\lesssim 5 \%$ ). This precision is a key advantage of modern CCD imaging over its photographic predecessor, since photographic brightness errors increase rapidly near the plate faint limit. Today, accurate, precise photometry is available over most of the sky, with typical faint limits near $m \sim 20$ mag. For example, SDSS has imaged $1 / 4$ of the sky to $r \sim 22$ and 2MASS imaged the entire sky to $J \sim 16.5$. The resulting databases contain accurate multi-band photometry of tens of millions of stars, enabling exciting new science. In particular, 2MASS photometry led to the discovery of two new spectral classes: L and T (Kirkpatrick et al., 1999) and was used to trace out the structure of the Sagittarius dwarf galaxy (Majewski et al., 2003). SDSS discovered the first field methane brown dwarf, which was the coolest substellar object known at the time of its discovery (Strauss et al., 1999). Other notable SDSS results have included the discovery of new stellar streams in the halo (e.g. Yanny et al., 2003; Belokurov et al., 2006) and new Milky Way companions (e.g., Willman et al., 2005; Belokurov et al., 2007), as well as unprecedented in situ mapping of the stellar density (Jurić et al., 2008) and metallicity (Ivezic et al., 2008) distributions of the Milky Way.

Furthermore, SDSS has produced the largest spectroscopic database to date, with 
over 1.2 million observations. It has yielded a wealth of information about the Universe on cosmological scales, as well as the local Milky Way. The SDSS spectroscopic sample has been used to map out the large scale structure of the Universe (e.g., Eisenstein et al., 2005; Richards et al., 2006) and to discover many of the most distant quasars (Fan et al., 2006, and references therein). At much smaller redshifts $(z=0)$, the local metallicity structure of the Milky Way (Ivezic et al., 2008) and magnetic activity lifetimes of M dwarfs (West et al., 2008) have been quantified with SDSS spectroscopy.

Motivated by the advantages of today's astronomical databases, my thesis will measure the average properties of $\mathrm{M}$ dwarfs, and use these stars to map the kinematic structure and stellar density of the local Milky Way. In the following section, I summarize the average spectroscopic and photometric properties of $\mathrm{M}$ dwarfs, followed by a discussion of their utility as tracers of Galactic populations in $\S 1.3$. In $\S 1.4$, I outline the current understanding of the field low-mass stellar luminosity function (LF) and mass function (MF). Finally, a brief outline of my dissertation is given in $\S 1.5$.

\subsection{Observational Properties of Low-Mass Dwarfs}

\subsubsection{Spectroscopic Features}

Spectroscopy provides a detailed observational description of a star's temperature, composition and gravity. Thus, the spectral type of a star is a proxy for its physical parameters: mass, effective temperature and metallicity. Spectral types are purely morphological and are usually based on features that vary smoothly across a large range of stellar parameters. For example, the hydrogen Balmer lines are weak in O stars, grow in strength through the B spectral subclass, peak through the A star subtype, and fall off thereafter.

For M dwarfs, the obvious choice for smoothly-varying spectral features are the 
prominent molecular bandheads found throughout the optical regime. The widely accepted Kirkpatrick et al. (1991) system incorporates spectral features from 6000$9000 \AA$ and is dominated by the strongest bandheads in this region, TiO, VO and CaH. Reid et al. (1995a) developed smooth spectral-type - TiO relations, which mimic the Kirkpatrick et al. (1991) sequence. Prior to the SDSS, the PMSU sample (Reid et al., 1995a; Hawley et al., 1996; Gizis et al., 2002; Reid et al., 2002) was the largest spectroscopic study of low-mass dwarfs, with $\sim 3,000$ stars drawn from a volumecomplete sample of low-mass dwarfs within 25 pc. The PMSU survey was based on observations in the CNS3 catalog (Gliese \& Jahreiss, 1991), a census of nearby stars, mostly selected by proper motion. PMSU characterized many properties of nearby low-mass stars, including chromospheric activity (Hawley et al., 1996; Gizis et al., 2002), kinematics (Reid et al., 1995a; Gizis et al., 2002) and the field LF and MF (Reid et al., 1995a, 2002).

With the advent of SDSS spectroscopy, large datasets of low-mass stellar spectra are readily available. Hawley et al. (2002) characterized the average spectral and photometric properties of M, L, and T dwarfs in the SDSS system from a sample of $\sim 700$ stars and brown dwarfs. West et al. (2004) and Bochanski et al. (2007b) extended these investigations, with samples of $\sim 8,000$ and $\sim 6,000 \mathrm{M}$ dwarfs, respectively. The West et al. (2004) study focused on magnetic activity and composition, and showed for the first time that chromospheric activity decreased with height above the Galactic Plane. They attributed this to an age-activity relation. West et al. (2004) also compared the average colors of low-metallicity subdwarfs to their solar-metallicity counterparts. Bochanski et al. (2007b) $)^{2}$ constructed a large set of high signal-to-noise spectra and coadded them to produce fiducial templates. The Bochanski et al. (2007b) templates were used to measured the average molecular bandhead indices at each subtype and study changes in spectral appearance due to

\footnotetext{
${ }^{2}$ This work is presented in Chapter 2 .
} 
activity and metallicity.

Currently, the largest spectroscopic sample of M dwarfs is the West et al. (2008) study, which analyzes SDSS observations of over 28,000 stars. Using simple dynamic heating arguments, along with the observed dependence of magnetic activity on height above the Plane (West et al., 2004), they estimated the magnetic activity lifetime of each spectral type. Their analysis shows a rapid increase in lifetime near spectral type M4, where low-mass stars are thought to become fully convective (Baraffe et al., 1998). Without the statistical power provided by the large datasets of SDSS spectroscopy, this type of study would be observationally prohibitive.

\subsubsection{Broadband Photometry}

While the spectrum of a star reveals a detailed picture of the star's temperature, structure and composition, it is observationally more expensive than broadband photometry. Spectroscopy samples a star's underlying spectral energy distribution (SED) at high resolution, while photometry uses wide $(\sim 1000 \AA)$ filters to roughly estimate the shape of the SED. Photometry in two filters is used to compute a color approximate the stellar SED and the star's temperature. The color is also used as a proxy of the star's absolute magnitude. The "observer's" Hertzsprung-Russell (HR) diagram $^{3}$ plots absolute magnitude as a function of color, for stars with known distances. Smooth functions are fit, and the resulting color-magnitude relations (CMRs) are applied to photometric observations to compute the stellar distances. CMRs and their application to SDSS photometry are covered in detail in Chapter 4.

The colors of low-mass stars in the SDSS ugriz system were first observationally quantified by Hawley et al. (2002). They determined the average color as a function of spectral type, and noted the rather large $(\sigma \sim 0.1)$ dispersion in color at a given

\footnotetext{
${ }^{3}$ The HR diagram is named after Ejnar Hertzsprung and Henry Norris Russell, stellar astronomers who independently developed the plot around 1910. By simply plotting the brightness of Pleiades and Hyades members against crude color estimates, they produced a significant development in the study of stellar evolution and structure.
} 
spectral type. These relations were subsequently updated by West et al. (2005), Bochanski et al. (2007b), Covey et al. (2007b) and West et al. (2008). Current research is ongoing (Kowalski et al., in preparation) to develop relations that use multiple colors to estimate the spectral type of a low-mass dwarf.

\subsection{Dwarfs \& the Local Milky Way: Low-Mass Stars as Tracers of Galactic Populations}

M dwarfs are long-lived (Laughlin et al., 1997) and ubiquitous, being found in each of the Galactic stellar populations (thin disk, thick disk and halo). Many of the low-mass dwarfs in the local solar neighborhood may have been formed elsewhere in Milky Way. However, the composition and kinematic properties of low-mass dwarfs should track their natal stellar population.

Traditionally, star counts have been used to measure the stellar density profile of the Milky Way. Stars are observed in multiple fields spread across the sky and a model is produced that matches the magnitude and color distributions that are observed. Although M dwarfs are the dominant stellar component of the Galaxy (by number), they have been used sparingly in star counts, due to the difficulty in observing them at large distances (e.g., Reid, 1993; Kroupa et al., 1993; Siegel et al., 2002).

While star-count modeling is a powerful technique, it is not always possible to find a unique solution, resulting in relatively weak constraints on the underlying stellar density profile. A more direct method simply measures densities of a complete sample along a line of sight. This approach has produced significant results. In particular, it was used to first demonstrate the presence of a "thick disk": Gilmore \& Reid (1983) measured the densities of G stars towards the South Galactic Pole and deduced the existence of a secondary disk component, with an exponential scale height larger than the well-known thin disk.

The stellar density technique has also been applied to SDSS photometry. Using a sample of F-M stars, Jurić et al. (2008) calculated three-dimensional stellar density 
maps of the Milky Way, extending to distances of a few kpc. Rather than simulate star counts, Jurić et al. (2008) were able to measure the shape of the Milky Way in situ and estimate the scale heights and lengths of the disk components, along with the halo. Since they are intrinsically dim, M dwarfs are only seen at small distances, ranging from roughly $2 \mathrm{kpc}$ for M0-M1 stars to less than $100 \mathrm{pc}$ for M8 and M9 dwarfs. Thus, they make ideal tracers of local Galactic structure. Using the largest photometric sample of M dwarfs ever assembled, I will directly measure the shape of the Milky Way, using a variation of the technique introduced by Jurić et al. (2008). This work is presented in detail in Chapter 5.

The local kinematic structure of the Galactic disk maintains the imprint of the original angular momentum of the proto-Galaxy. As the disk formed, the angular momentum of this rotating structure was conserved, producing the ordered rotation observed in the Milky Way. Motion is usually measured in the $U V W$ coordinate system (Johnson \& Soderblom, 1987), where $U$ is positive in the direction of the Galactic center, $V$ is positive in the direction of the Galactic rotation, and $W$ is positive north of the Galactic plane. Local kinematic structure is usually described by velocity dispersions with respect to these axes, although deviations of the best-fit ellipsoid are observed (Dehnen \& Binney, 1998). Parenago (1950) was the first to observe the large increase in the velocity dispersions of late-type dwarfs when compared to early spectral types. "Parenago's discontinuity" results from a convolution of shorter stellar lifetimes at earlier spectral types and dynamic heating due to interactions with giant molecular clouds and spiral density waves.

Modern kinematic surveys can trace their roots back to earlier proper motion surveys, such as Giclas et al. (1971) and Luyten (1979). The largest kinematic sample of M dwarfs prior to SDSS was PMSU (Reid et al., 1995a; Hawley et al., 1996; Gizis et al., 2002; Reid et al., 2002). Using proper motions and radial velocities, Reid et al. (1995a) and Hawley et al. (1996) showed that magnetically active stars were kinematically colder (e.g., Wielen, 1977a). Since activity is thought to trace stellar age 
(Wielen, 1977a; West et al., 2008), the younger, active M dwarfs have not undergone as many interactions in the disk, resulting in smaller velocity dispersions. This behavior was also observed by Bochanski et al. (2005), who studied a sample of stars within 100 pc of the Sun.

With the fainter magnitude limits and multi-object capabilities of the SDSS spectrograph, I am poised to extend this investigation to larger distances, sampling both the thin and thick disks. Measuring the changes in the velocity ellipsoid of $\mathrm{M}$ dwarfs with respect to the Plane directly tests the predictions of current Galactic kinematic models. Moreover, it constrains the kinematic properties of the thin and thick disk. The large sample sizes needed to accurately measure the velocity dispersion in small bins of vertical height were not available prior to the SDSS survey. I detail my investigation into the kinematic structure of the local Milky Way in Chapter 3.

\subsection{Field Luminosity and Mass Functions of Low-Mass Stars}

Characterizing the number density of low-mass stars, either by luminosity (Chapter 5) or mass (Chapter 6) has a rich astronomical history. The stellar luminosity function:

$$
\Phi(M)=d N / d M
$$

is defined as the number of stars per unit absolute magnitude per unit volume. It summarizes the observed distribution of absolute magnitude of a given sample. When combined with a mass-luminosity relation (MLR), the luminosity function can be directly transformed to the stellar mass function:

$$
\psi(\mathrm{M})=d N / d \mathrm{M}
$$


the number of stars per unit mass per unit volume ${ }^{4}$. The mass function succinctly describes the fundamental mass spectrum produced during star formation events, the initial mass function (IMF). For low-mass stars, with lifetimes much greater than the Hubble time, the observed present-day mass function (PDMF) in the field is the $\mathrm{IMF}^{5}$. Since the mass of a star dictates the majority of its physical properties, accurate observational measurements of the low-mass IMF impose strong constraints on stellar evolution and star formation theory.

The low-mass IMF plays a crucial role across a wide spectrum of astronomical topics. Since low-mass stars have long main sequence lifetimes and dominate the Galactic stellar populations (by number), they influence Galactic chemical evolution, acting as mass sinks. The measured MF is also used to constrain the masses of external galaxies, through stellar mass-to-light ratios (e.g. Bell \& de Jong, 2001). The IMF even impacts planetary science, as it characterizes the population of stars near the Sun, which have potentially triggered comet showers in the past (Kaib \& Quinn, 2007).

Previous investigations of the low-mass luminosity function (LF) and mass function (MF) were limited to deep, pencil beam surveys with limited areal coverage (e.g., Zheng et al., 2001), or volume-complete samples over wide solid angles, but only to distances of $\lesssim 20$ pc (e.g., Reid \& Gizis, 1997). Sample sizes were limited to a few thousand stars, prohibiting a detailed determination of the IMF. In general, the IMF has been characterized by a power-law $\psi(\mathrm{M})=d N / d \mathrm{M} \propto \mathrm{M}^{-\alpha}$, with the exponent $\alpha$ varying over a wide range from $0.5-2.5$ (see Table 6.1 for details).

Using a sample drawn from SDSS, 2MASS and Guide Star Catalog photometry and supplemented with SDSS spectroscopy, Covey et al. (2008) performed the largest field low-mass LF and MF investigation to date. Composed of $\sim 30,000$ low-mass

\footnotetext{
${ }^{4}$ Throughout this dissertation, I will refer to absolute magnitude with $M$, while I will use M when indicating mass.

${ }^{5}$ Although this is not strictly true in young clusters.
} 
stars over $30 \mathrm{sq}$. deg, their sample measured the LF using absolute magnitudes estimated from photometric colors, and quantified the contamination rate by obtaining spectra of every red point source in a 1 sq. degree calibration region. The Covey et al. (2008) sample serves as a calibration study for this dissertation, as it quantified the completeness, bias and contamination rate of the SDSS and 2MASS photometric samples. While their study focused on one sightline, my dissertation expands to the entire SDSS footprint, increasing the solid angle by a factor of $\sim 300$. This work is detailed in Chapters 5 and 6.

\subsection{Thesis Outline}

My dissertation proceeds with a discussion of the average spectroscopic properties and broadband colors of $\mathrm{M}$ dwarfs in Chapter 2. The radial velocity templates developed from this sample are applied to a targeted set of SDSS spectra in Chapter 3, and the resulting $U V W$ velocities are used to trace out the local Milky Way kinematic structure. Chapter 4 details my investigation into the color-absolute magnitude relations of low-mass stars in the SDSS ugriz photometric system. The resulting CMRs are used to derive distances to $\sim 15$ million low-mass stars observed photometrically by SDSS. Stellar density maps are computed to measure the local Galactic structure, in Chapter 5. These maps are constructed as a function of absolute magnitude and are used to determine the field luminosity function of low-mass stars with a sample size three orders of magnitude larger than any previous study. The resulting LF, discussed

in Chapter 5, is transformed into the field mass function in Chapter 6, and compared to previous works. Finally, my conclusions and possible future investigations are outlined in Chapter 7. 
Chapter 2

\section{LOW-MASS DWARF TEMPLATE SPECTRA FROM THE SLOAN DIGITAL SKY SURVEY}

\section{$2.1 \quad$ Chapter Summary}

We present template spectra of low-mass (M0-L0) dwarfs derived from over 4,000 Sloan Digital Sky Survey (SDSS) spectra. These composite spectra are suitable for use as medium-resolution $(\mathrm{R} \sim 1,800)$ radial velocity standards. We report mean spectral properties (molecular bandhead strengths, equivalent widths) and use the templates to investigate the effects of magnetic activity and metallicity on the spectroscopic and photometric properties of low-mass stars.

Sections 2.2 through 2.6 of this chapter was originally published in collaboration with Andrew A. West, Suzanne L. Hawley, and Kevin R. Covey in the February 2007 edition of the Astronomical Journal (Bochanski et al., 2007b, AJ Vol. 133, pp. 531; (c) 2007 by the American Astronomical Society) and is reproduced below with permission of the American Astronomical Society.

\subsection{Introduction}

Low-mass dwarfs are the dominant stellar component of the Galaxy. These ubiquitous stars, with main sequence lifetimes greater than the Hubble time (Laughlin et al., 1997), have been employed in a variety of Galactic studies: tracing Galactic Disk kinematics (Hawley et al., 1996; Gizis et al., 2002; Lépine et al., 2003a; Bochanski et al., 2005), describing age-velocity dispersion relations (West et al., 2006), and studying Galactic structure components (Reid et al., 1997; Kerber et al., 2001; Pirzkal et al., 2005). Modelling of their internal structure, atmospheric properties and mag- 
netic activity (Burrows et al., 1993; Baraffe et al., 1998; Allard et al., 1997; Hauschildt et al., 1999; Allred et al., 2006; West et al., 2006) presents interesting theoretical problems. Observationally, the spectra of these stars are marked by the presence of strong molecular absorption features, particularly titanium oxide ( $\mathrm{TiO})$, which dominates the optical opacity of their cool atmospheres. The $\mathrm{TiO}$ features, along with molecular bandheads introduced by vanadium oxide (VO) and calcium hydride (CaH) are used to define the widely accepted M spectral subtype classification scheme (Kirkpatrick et al., 1991; Reid et al., 1995a; Kirkpatrick et al., 1999).

In order to increase the utility of low-mass stars in large studies of galactic structure and dynamics, we have been engaged in analyzing spectroscopic data from the Sloan Digital Sky Survey (SDSS; York et al., 2000), resulting in a series of papers that describe our methods for photometrically selecting and spectral typing these objects (Hawley et al., 2002; West et al., 2004; Walkowicz et al., 2004; West et al., 2005) and discussing their magnetic activity properties (West et al., 2004, 2006). However, we have been unable to exploit the velocity information contained in the spectra due to the inability of the SDSS pipeline reductions to provide accurate velocites for $\mathrm{M}$ dwarfs (Abazajian et al., 2004). Our motivation for the present work is the desire to produce a set of radial velocity templates by combining native, high-quality, SDSS spectra at each spectral subtype in the $\mathrm{M}$ dwarf sequence. Additionally, we split the spectra at each subtype into active and inactive stars, and examine the spectroscopic and photometric properties of these templates separately, to determine whether the activity is imprinting a signature that may affect our velocity analysis, and to followup on previous suggestions that colors and detailed absorption features may change depending on activity level (Hawley et al., 1996; Amado \& Byrne, 1997; Hawley et al., 1999).

The radial velocity $(\mathrm{RV})$ of an object is the projection of its intrinsic motion onto the line of sight of an observer. In order to accurately determine the RV of a given star, one must carefully address the systematics imposed by the time and location of 
the observation. This is usually accomplished by shifting the frame of the observer to a heliocentric (sun-centered) or barycentric (center-of-mass centered) rest frame. The standard method of determining stellar and galactic RVs has been cross-correlation, as introduced by Tonry \& Davis (1979). This method compares the spectrum of a science target against a known template, using cross-correlation to determine the wavelength shift (and therefore velocity) necessary to align the target with the template. Thus, correlating with a template spectrum that is similar to the science target in all ways except velocity ensures the most accurate determination of the RV.

In the following sections, we report on our efforts to establish a set of low-mass star template spectra ${ }^{1}$ suitable for RV analysis using SDSS spectra at medium $(\mathrm{R} \sim$ $1,800)$ resolution. In $\S 2.3$, we describe the observational material from the SDSS and introduce the problems with the RVs reported for low-mass stars by the standard SDSS spectroscopic pipeline. The observations were spectral-typed, inspected for signs of chromospheric activity, and coadded to form templates at each spectral type, as discussed in $\S 2.4$. The resulting spectral templates, their accuracy as RV standards, and their spectral and photometric properties are detailed in $\S 2.5$. Our conclusions follow in $\S 2.6$.

\subsection{Data}

\subsubsection{SDSS Photometry}

The Sloan Digital Sky Survey (York et al., 2000; Gunn et al., 1998; Fukugita et al., 1996; Hogg et al., 2001; Smith et al., 2002; Stoughton et al., 2002; Abazajian et al., 2003; Pier et al., 2003; Abazajian et al., 2004; Ivezić et al., 2004; Abazajian et al., 2005; Adelman-McCarthy et al., 2006; Gunn et al., 2006) has revolutionized optical astronomy. Centered on the Northern Galactic Cap, SDSS has photometrically imaged $\sim 8,000$ sq. deg. in five filters $(u, g, r, i, z)$ to a faint limit of 22.2 mag in $r$.

\footnotetext{
${ }^{1}$ Available at http://www.astro.washington.edu/slh/templates/
} 
This has resulted in photometry of $\sim 180$ million objects with typical photometric uncertainties of $\sim 2 \%$ at $r \sim 20$ (Ivezić et al., 2003; Adelman-McCarthy et al., 2006). SDSS imaging has been invaluable in recent studies concerning low-mass dwarfs, particularly the colors of the M star sequence (Walkowicz et al., 2004; West et al., 2005) and the study of L and T spectral types (Strauss et al., 1999; Fan et al., 2000; Leggett et al., 2000; Tsvetanov et al., 2000; Hawley et al., 2002; Knapp et al., 2004; Chiu et al., 2006).

\subsubsection{SDSS Spectroscopy}

Photometry acquired in SDSS imaging mode is used to select spectroscopic followup targets. The photometry is analyzed by a host of targeting algorithms (originally described in Stoughton et al., 2002) with the primary spectroscopic targets being galaxies (Strauss et al., 2002), luminous red galaxies with $\mathrm{z} \sim 0.5-1.0$ (Eisenstein et al., 2001), and high redshift quasars (Richards et al., 2002). Designed to acquire redshifts for $\sim 1,000,000$ galaxies and 100,000 quasars, twin fiber-fed spectrographs deliver 640 flux-calibrated spectra per $3^{\circ}$ diameter plate over a wavelength range of $3800-9200 \AA$ with a resolution $R \approx 1,800$. Typical observations are the coadded result of multiple 15 minute exposures, with observations continuing until the signal-to-noise ratio per pixel is $>4$ at $g=20.2$ and $i=19.9$ (Stoughton et al., 2002). Typically,

this takes about 3 exposures. Wavelength calibrations, good to $5 \mathrm{~km} \mathrm{~s}^{-1}$ or better (Adelman-McCarthy et al., 2006), are carried out as described in Stoughton et al. (2002). The spectra are then flux-calibrated using F subdwarf standards, with broadband uncertainties of $4 \%$ (Abazajian et al., 2004). These observations, obtained and reduced in a uniform manner, form a homogeneous, statistically robust dataset of over 673,000 spectra (Adelman-McCarthy et al., 2006). SDSS has already proven to be an excellent source of low-mass stellar spectroscopy (Hawley et al., 2002; Raymond et al., 2003; West et al., 2004; Silvestri et al., 2006). Unfortunately, the RVs determined for 
low-mass stars in the SDSS pipeline are known to be inaccurate ${ }^{2}$ (Abazajian et al., 2004). These systematic errors, on the order of $10 \mathrm{~km} \mathrm{~s}^{-1}$ (Abazajian et al., 2004) result primarily from spectral mismatch, since there are only four low-mass stellar templates in the standard spectroscopic pipeline. Thus, we sought to remedy this situation by establishing a uniform set of low-mass RV templates derived from SDSS spectroscopy.

\subsection{Analysis}

To build a database of low-mass stellar spectra, we queried the Data Release 3 (DR3; Abazajian et al., 2005) Catalog Archive Server (CAS) for spectra with late-type dwarf colors (from West et al., 2005), using $0.5<r-i<3.05$ and $0.3<i-z<1.9$. The color ranges quoted in West et al. (2005) were slightly extended to increase the total number

of low-mass stellar spectra. These color cuts were the only restrictions applied to the DR3 data. We treated each spectral subtype independently, performing 11 (M0-L0) queries, some of which overlapped in color-color space (see Table 2.1). Thus, some spectra were selected twice, usually in neighboring spectral subtypes (i.e., M0 and M1). These queries yielded $\sim 133,000$ candidate spectra in the 11 (M0-L0) spectral type bins.

\subsubsection{Spectral Types and Activity}

The candidate spectra were examined with a suite of software specifically designed to analyze M dwarf spectra. This pipeline, as introduced in Hawley et al. (2002), measures a host of molecular band indices (TiO2, TiO3, TiO4, TiO5, TiO8, CaH1, $\mathrm{CaH} 2$, and $\mathrm{CaH} 3$ ), and employs relations first described by Reid et al. (1995a) to determine a spectral type from the strength of the TiO5 bandhead. All spectral types were confirmed by manual inspection, adjusting the final spectral type, if necessary.

\footnotetext{
${ }^{2}$ See http://www.sdss.org/dr5/products/spectra/radvelocity.html
} 
The accuracy of the final spectral type is \pm 1 subtype. Additionally, the software pipeline measures the equivalent width $(\mathrm{EW})$ of the $\mathrm{H} \alpha$ line, quantifying the level of magnetic activity in a given low-mass dwarf. See West et al. (2004) for details on the measurement of bandheads and line strengths in low-mass star SDSS spectra.

Inspection of each candidate spectrum allowed us to remove contaminants (mostly galaxies) from the sample, reducing its size to $\sim 20,000$ stellar spectra. We also removed the white dwarf-M dwarf pairs that were photometrically identified by Smolčić et al. (2004). The database was then culled of duplicates. As shown in West et al. (2005) (and Table 2.1), M dwarfs of different spectral types can possess similar SDSS photometric colors. Thus, some spectra with overlapping photometric colors were duplicated in our original database (see $\S 2.4$ and Table 2.1). Each duplicate spectrum was identified by filename and in cases where different spectral types were manually assigned by eye to the same star (typically one subtype apart), the earlier spectral type was kept. The typical difference in spectral type was one subclass, in agreement with our stated accuracy. These various cuts reduced the sample from $\sim 20,000$ to $\sim 12,000$ stars.

The spectra were then categorized based on their activity. In order to be considered active, a star had to meet the criteria originally described in West et al. (2004): (1) The measured $\mathrm{H} \alpha \mathrm{EW}$ is larger than $1.0 \AA$; (2) The measured EW is larger than the error; (3) The height of the $\mathrm{H} \alpha$ line must be three times the noise at line center; (4) The measured EW must be larger than the average EW in two $50 \AA$ comparison regions (6500-6550 $\AA$ and $6575-6625 \AA$ ). In order to be considered inactive, the measured EW had to be less than $1.0 \AA$ and have a signal-to-noise ratio greater than three in the comparison regions. By selecting only these active and inactive stars, our final sample is limited to spectra with well-measured features, removing spectra with low signal-to-noise ratios. The resulting database consisted of $\sim 6,000$ stellar spectra. 


\subsubsection{Coaddition}

SDSS spectra are corrected to a heliocentric rest frame during the standard pipeline reduction and are on a vacuum wavelength scale. To assemble the fiducial template spectra, stars of a given spectral type were first shifted to a zero-velocity rest frame, then normalized and coadded. Multiple strong spectral lines were used to measure the velocity of each star to obtain an accurate shift to the rest frame. For inactive stars, the red line $(7699 \AA)$ ) of the K I doublet and both lines of the Na I doublet (8183, $8195 \AA$ ) were measured, while in active stars, $\mathrm{H} \alpha$ was also used. These spectral line combinations were selected for their strength in all low-mass stellar spectra, ensuring that no single line would determine the final velocity of a star. Spectral lines were fit with single Gaussians and inspected visually to ensure proper fitting. Stars with spurious fits or discrepant line velocities (lines which deviated from the mean by $>30$ $\mathrm{km} \mathrm{s}^{-1}$ ) were removed from the final coaddition. Removing these spectra reduced the final sample size to $\sim 4,300$ stars. The classical redshift correction was then applied to each spectrum in the final sample, justifying them to a zero-velocity rest frame.

The velocity of each spectral line in the final sample was fit with an measurement uncertainty of $\sim 10 \mathrm{~km} \mathrm{~s}^{-1}$, as determined by the mean scatter among individual line RV measurements. In wavelength space, this translates to about $0.2 \AA$ resolution near $\mathrm{H} \alpha$ (note the resolution of $\operatorname{SDSS}(\mathrm{R} \sim 1,800)$ implies $3.6 \AA$ resolution at $\mathrm{H} \alpha$ ). Since the observed spectrum is a discretization of a continuous flux source (i.e., the star), wavelength shifts introduced by the radial velocity of a star will move flux within and between resolution elements. These wavelength shifts, which are resolved to subpixel accuracy, act to increase the resolution of the final co-added spectrum (see Pernechele et al., 1996). This is similar to the common "drizzle" technique of using multiple, spatially distinct low-resolution images to produce a single high-resolution image (Fruchter \& Hook, 2002).

The wavelength-justified spectra were then normalized at $8350 \AA$ and coadded 
(with equal weighting of each spectrum) using the following prescription. At each subtype, we attempted to construct three templates: one composed solely of active stars (Fig. 2.1), one composed of inactive stars (Fig. 2.2), and a third composed of both the inactive and active stars from the previous two sets (Fig 2.3). For each template, the mean and standard deviation were calculated at each pixel. In later (> M7) subtypes, the lack of spectra meeting our activity and velocity criteria resulted in fewer templates. 


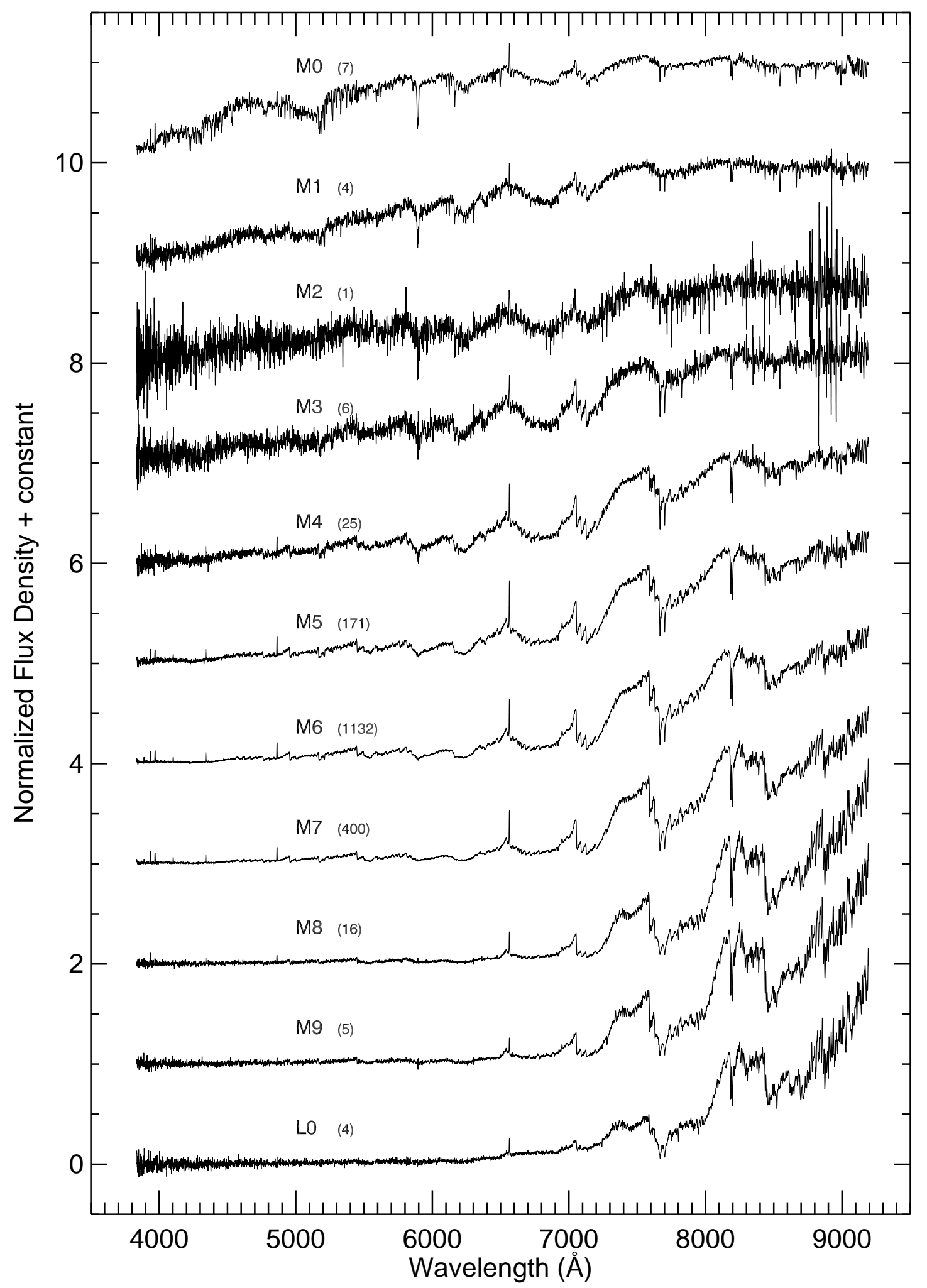

Figure 2.1 Mean template spectra of active low-mass dwarfs of types M0-L0. The spectral type and number of stars (in parentheses) are labeled for each template. 


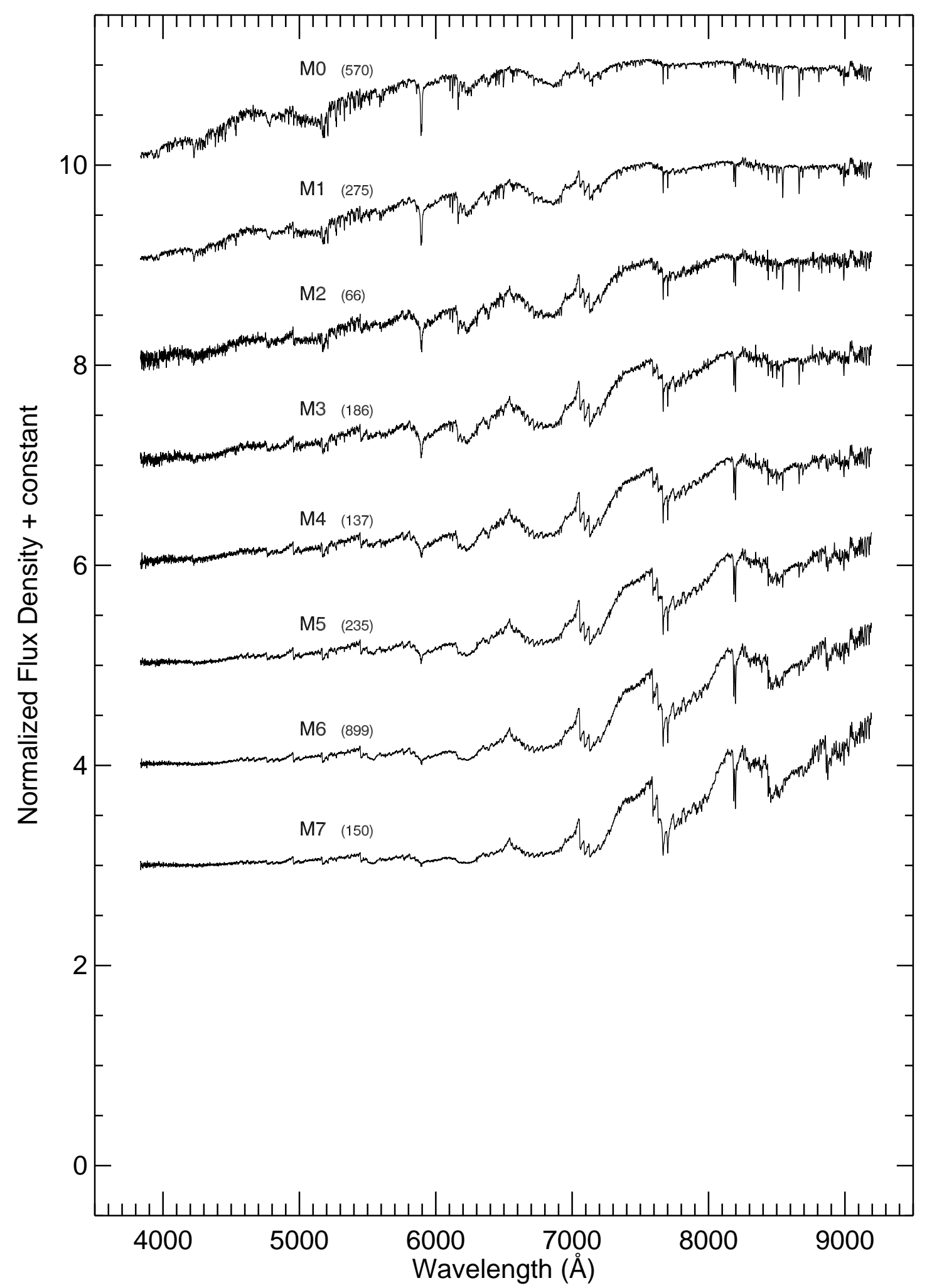

Figure 2.2 Mean template spectra of inactive low-mass dwarfs of types M0-M7. No M8-L0 dwarfs met the activity and consistent line velocity criteria. Spectral type and number of stars in each template are labeled. 


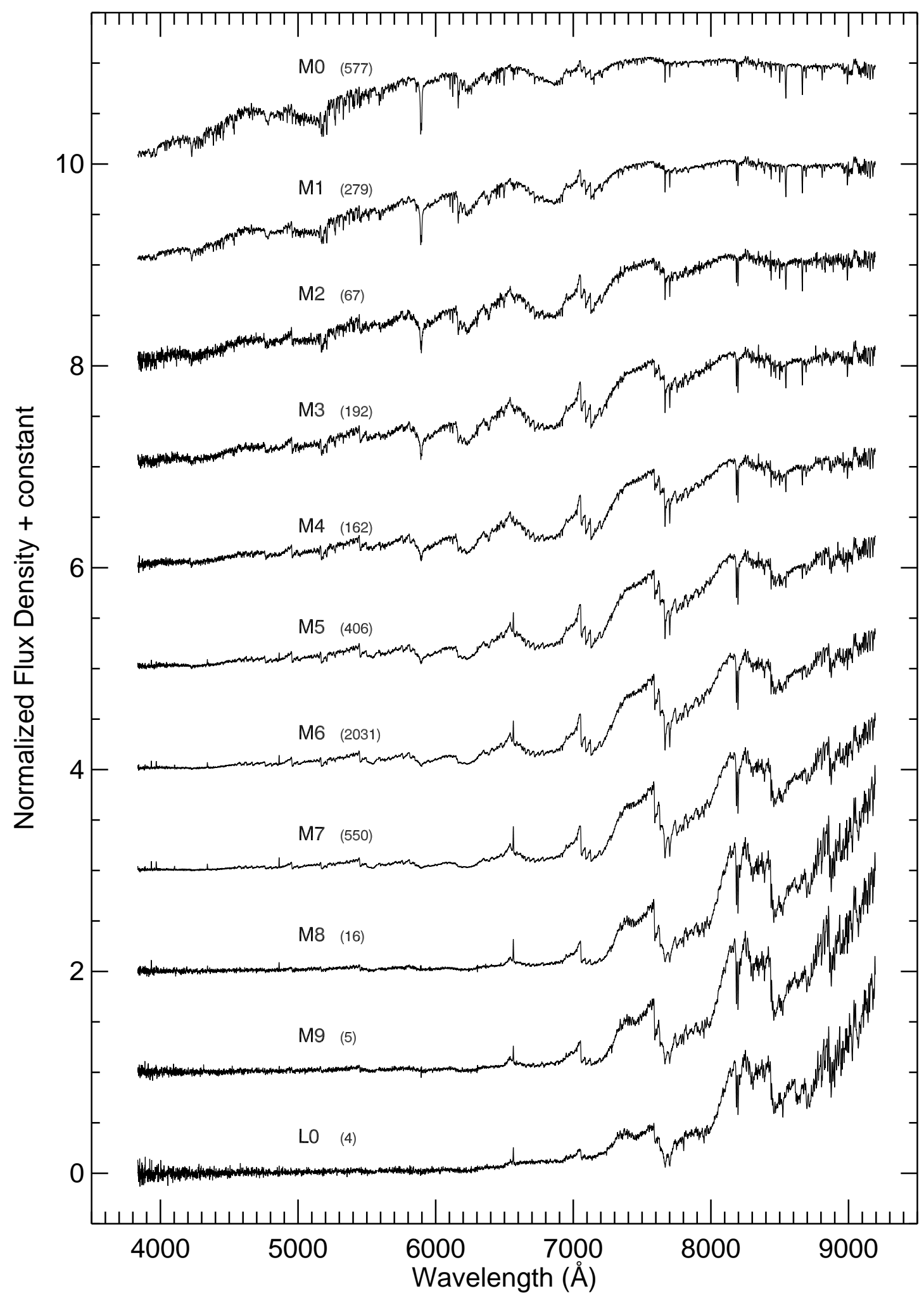

Figure 2.3 Mean composite template spectra for low-mass dwarfs of types M0-L0, formed by combining the data shown in Figures 1 and 2. Spectral type and number of stars in each template are labeled. 


\subsection{Results and Discussion}

The final template spectra (Figures 2.1-2.3) represent the mean spectral properties of low-mass dwarfs as observed by the SDSS spectrographs. In Figure 2.4, we show illustrative examples of our templates for an inactive M1 star and an active M6 star, with strong atomic lines and molecular bandheads labeled. Prominent molecules include $\mathrm{MgH}, \mathrm{CaH}, \mathrm{TiO}, \mathrm{VO}$ and $\mathrm{CaOH}$. The active stars show the Balmer series to H8 $(\lambda \sim 3889 \AA)$ along with Ca II H and $\mathrm{K}(\lambda \sim 3968,3933 \AA)$. In Figure 2.5 we compare a high signal-to-noise SDSS spectrum of an M5 star to its template counterpart in the region near $\mathrm{H} \alpha$. It is clear that the template has significantly higher spectral resolution; e.g. a weak feature near $6575 \AA$ is visible only in the template. In the following sections, we explore the feasibility of using these templates as RV standards and the effects of chromospheric activity and metallicity on the mean spectroscopic and photometric characteristics of low-mass stars.

\subsubsection{Radial Velocity Accuracy}

The primary uncertainties associated with determining RVs using the cross-correlation method are due to the resolution of the spectra, accuracy of the wavelength calibration and matching the spectral type of the template and science data. To ensure that the RVs measured with our templates are accurate, we have carried out tests that quantify the internal consistency and external zero-point precision of these templates. These tests are described below.

\section{Internal Consistency}

To quantify the internal consistency among templates, sequential spectral types were cross-correlated using the fxcor task in $\mathrm{IRAF}^{3}$. This minimizes the error introduced

\footnotetext{
${ }^{3}$ IRAF is distributed by the National Optical Astronomy Observatories, which are operated by the Association of Universities for Research in Astronomy, Inc., under cooperative agreement with the National Science Foundation.
} 

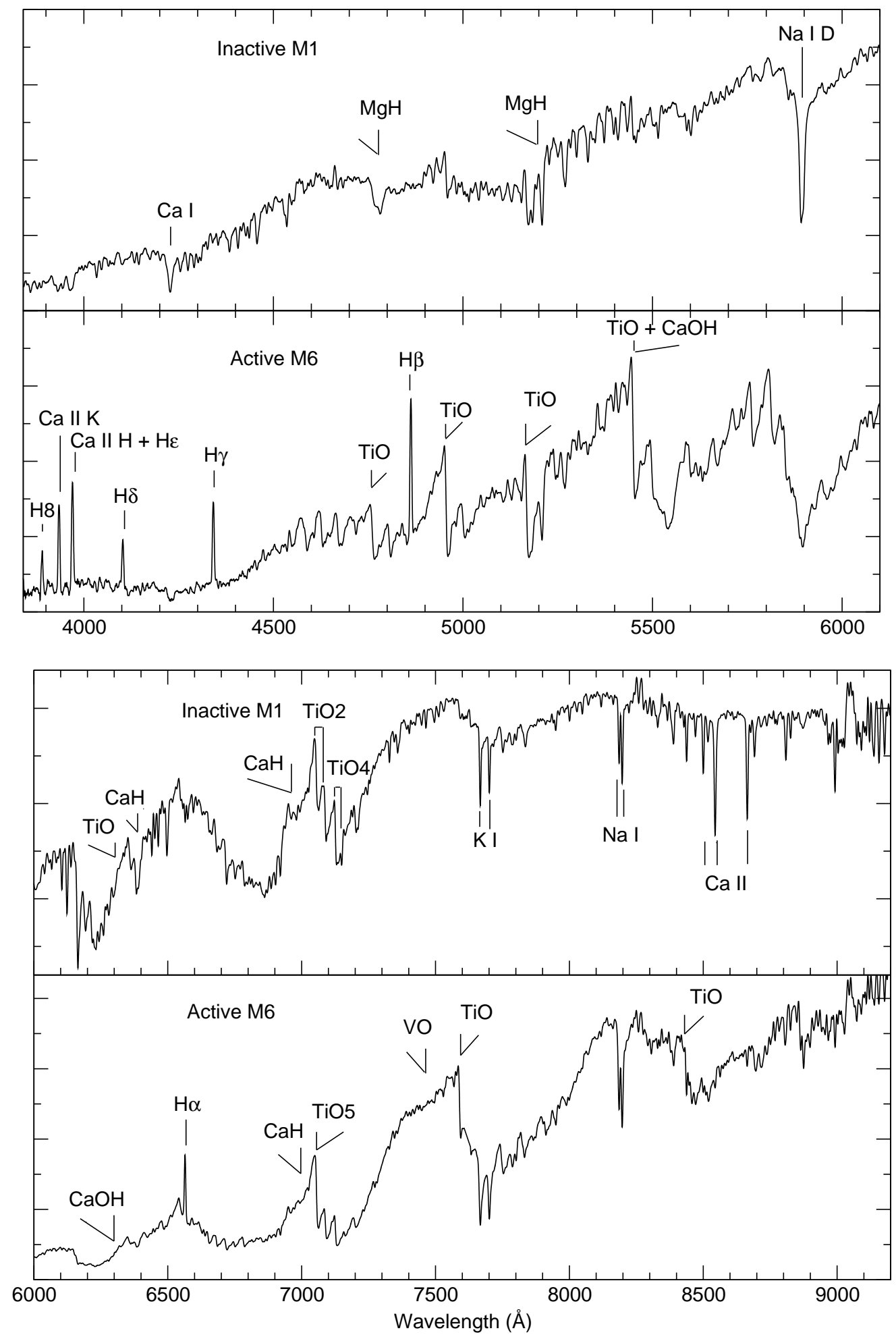

Figure 2.4 Illustrative template spectra of an inactive M1 star and an active M6 star with strong molecular and atomic features labeled. 


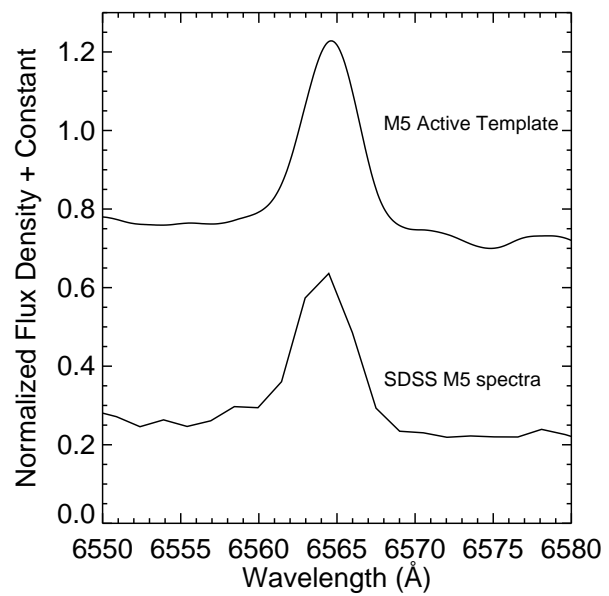

Figure 2.5 Comparison of a high signal-to-noise SDSS M5 spectrum (bottom) to the active M5 template (top) near $\mathrm{H} \alpha$. Note the higher resolution of the template spectrum.

by spectral type mismatch, which often dominates the errors associated with crosscorrelation redshift measurements (Tonry \& Davis, 1979). Thus, wavelength calibration and intrinsic resolution are the major sources of uncertainty in our analysis. In all cases (i.e., active, inactive and combined templates) the mean difference in velocity between adjacent spectral subtypes was $\lesssim 1 \mathrm{~km} \mathrm{~s}^{-1}$. This compares favorably with the $3.5 \mathrm{~km} \mathrm{~s}^{-1}$ spread in SDSS data as reported by York et al. (2000). Note this value is derived from observations of stars in M67 (Mathieu et al., 1986), and does not include any low-mass dwarfs.

\section{External Consistency: Hyades}

To test the external accuracy of the template spectra, they were cross-correlated against Hyades cluster members with well-measured RVs, observed as part of our SDSS collaboration effort to produce RV standards for low-mass dwarfs. Each Hyades star has a known RV (Reid \& Mahoney, 2000; Stauffer et al., 1994, 1997; Terndrup et al., 2000; Griffin et al., 1988) or is a confirmed member of the cluster, whose dispersion is $<1 \mathrm{~km} \mathrm{~s}^{-1}$ (Gunn et al., 1988; Makarov et al., 2000). The Hyades RVs 
in the literature were measured from high-resolution echelle spectra, with a typical accuracy of $\lesssim 1 \mathrm{~km} \mathrm{~s}^{-1}$. Thus, the SDSS spectra of these Hyads provide a way to check our cross-correlation RVs against an external standard system.

The medium-resolution spectra of the Hyades stars secured by SDSS were correlated against our templates, with results shown in Figure 2.6. The high-resolution echelle data have a mean of $38.8 \mathrm{~km} \mathrm{~s}^{-1}$ and a standard deviation of $0.27 \mathrm{~km} \mathrm{~s}^{-1}$. The RVs measured with our template spectra yield a mean RV of $42.6 \mathrm{~km} \mathrm{~s}^{-1}$ with a standard deviation of $3.2 \mathrm{~km} \mathrm{~s}^{-1}$. By comparison, the SDSS pipeline RVs produced a mean velocity of $31.9 \mathrm{~km} \mathrm{~s}^{-1}$ and a standard deviation of $6.8 \mathrm{~km} \mathrm{~s}^{-1}$ (after removing two highly discrepant measurements). Using the template spectra better reproduces the coherent velocity signature of the Hyades and provides much more reliable velocities than the standard SDSS pipeline measurements. The templates are therefore well-suited for use as medium-resolution RV standards for low-mass dwarfs.

\subsubsection{Spectral Differences: Activity 8 Metallicity}

The effect of magnetic activity on the spectral properties of a low-mass star is clearly manifested by the existence of emission lines. This effect is often quantified by measuring the luminosity in the $\mathrm{H} \alpha$ line divided by the bolometric luminosity $\left(L_{\mathrm{H} \alpha} / L_{\mathrm{Bol}}\right)$. Other changes due to activity, such as varying strength of molecular bandheads (Hawley et al., 1996, 1999) and changes in the shape of the continuum have been sparsely investigated. Additionally, metallicity affects the strength of molecular bandheads at a given temperature (see Woolf \& Wallerstein, 2006). Using the template spectra as fiducial examples of thin-disk, solar-metallicity low-mass stars, we next examine changes in the spectral properties of low-mass stars introduced by magnetic activity and metallicity. 


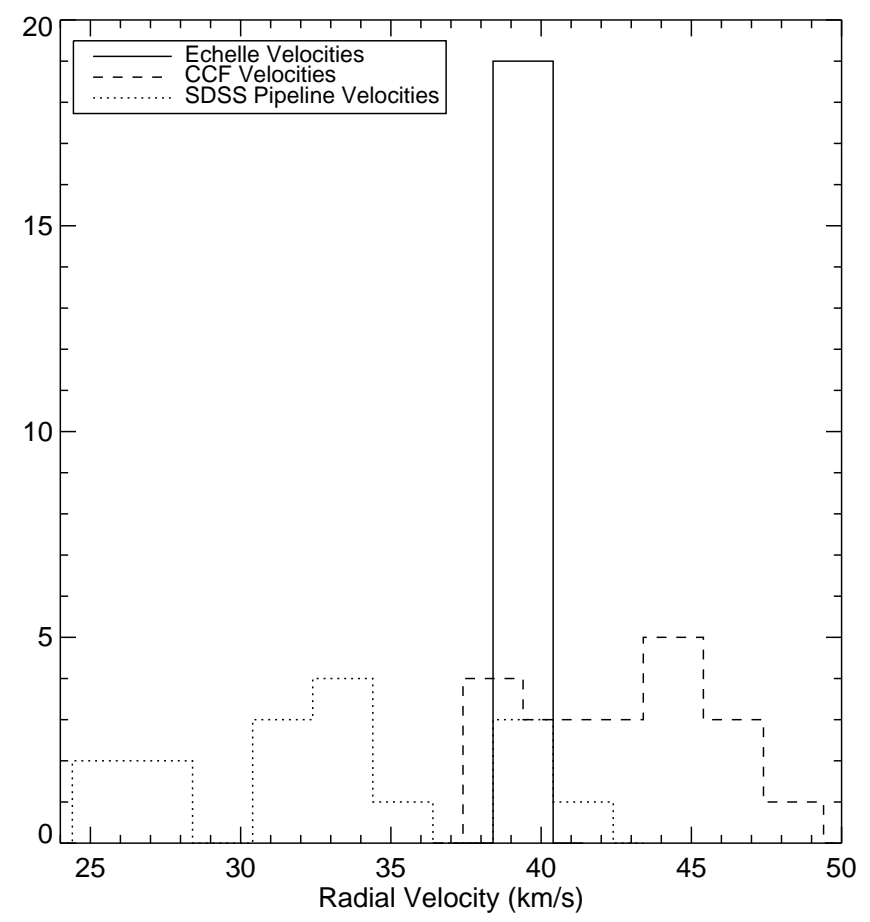

Figure 2.6 Histogram comparison of high-resolution echelle RV measurements (solid line), medium resolution cross-correlation RV determinations (dashed line), and SDSS pipeline RVs (dotted line) for 19 Hyades M dwarfs. Note the SDSS pipeline reported wildly discrepant velocities for two stars (not shown). 
Activity: Decrements \& $L_{\mathrm{H} \alpha} / L_{\mathrm{Bol}}$

Emission features are dependent on the temperature and density structure of the outer stellar atmosphere. The line fluxes of the Balmer series lines and the Ca II $\mathrm{K}$ line $(\lambda \sim 3933 \AA)$ can be used to examine the structure of the chromosphere in magnetically active stars (Reid et al., 1995b; Rauscher \& Marcy, 2006) and to investigate chromospheric heating in quiescent (i.e. non-flaring) dMe stars (Mauas \& Falchi, 1994; Mauas et al., 1997). The Balmer decrement (ratios of Balmer line strengths to a fiducial, here taken to be $\mathrm{H} \beta$ ) is traditionally used to quantify mediumresolution spectra. Table 2.2 gives the Balmer series and Ca II K decrements for the active templates. There is no strong trend in the decrement with spectral type for the template spectra, which is consistent with the previous study of Pettersen \& Hawley (1989), who reported average decrements over a range of $\mathrm{K}$ and $\mathrm{M}$ spectral types. However, both the templates and Pettersen \& Hawley (1989) show a gradual increase in the $\mathrm{H} \alpha / \mathrm{H} \beta$ ratio with spectral type (see Table 2.2). Evidently the structure and heating of low-mass stellar chromospheres remains fairly similar over the range of $\mathrm{M}$ dwarf effective temperature (mass), with the $\mathrm{H} \alpha$ gradually becoming stronger relative to the higher order Balmer lines at later spectral type.

The Balmer decrements observed in AD Leo (dM3e) during a large flare (Hawley \& Pettersen, 1991) and determined for quiescent and flaring model atmospheres (Allred et al., 2006) are given in Table 2.2 for comparison. In Figure 2.7, we plot these observed and model decrements together with the average Balmer decrements of the active low-mass stellar templates. The flare decrements, both observed and model, are much flatter than those in the non-flaring atmospheres, suggesting (though within the errors) increased emission in the higher order Balmer lines. This probably reflects the higher chromospheric densities (hence greater optical depth in the Balmer line-forming region) in the flare atmospheres. Evidently the range in chromospheric properties among M dwarfs of different spectral types is much less (during quiescent periods) 
than in a given star between its quiet and flaring behavior.

For completeness, the average Balmer line and Ca II K EWs and the quantity $L_{\mathrm{H} \alpha} / L_{\mathrm{Bol}}$ measured from the active templates are reported in Table 2.3. $L_{\mathrm{H} \alpha} / L_{\mathrm{Bol}}$ which is used to quantify activity, was calculated using the $\mathrm{H} \alpha \mathrm{EW}$ and the $(i-z)$ continuum $(\chi)$ relation of West et al. (2005), as first described in Walkowicz et al. (2004). The average EWs reported in Table 2.3 agree with previous results (Stauffer et al., 1997; Gizis et al., 2002). The general increase toward later types is attributed to the lower continuum flux in the vicinity of $\mathrm{H} \alpha$ as the stellar effective temperature decreases. The $L_{\mathrm{H} \alpha} / L_{\mathrm{Bol}}$ ratios are also consistent with previous studies (Hawley et al., 1996; Gizis et al., 2000; West et al., 2004), attaining a relatively constant value (with large scatter) among early-mid M (M0-M5) types, and decreasing at later types.

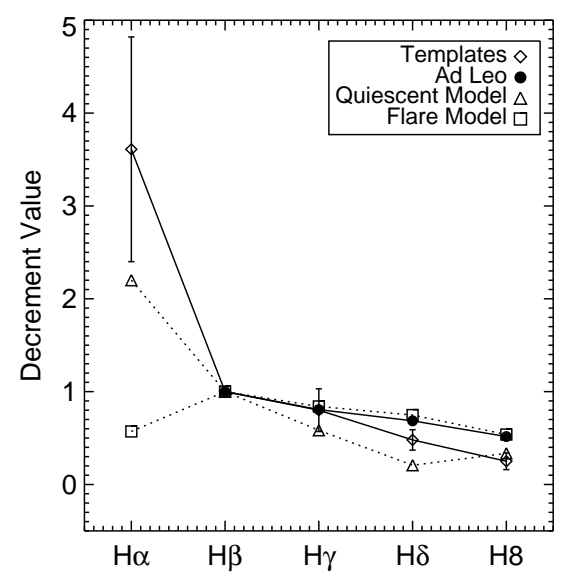

Figure 2.7 Decrements for the averaged active templates (open diamonds, error bars) are shown. Also shown are results for the dM3e star AD Leo: observed flare (filled circles; from Hawley \& Pettersen, 1991), model flare (open squares), and model quiescent (open triangles) decrements (Allred et al., 2006). Flaring atmospheres have increased density at chromospheric temperatures, resulting in higher opacity and increased emission in the higher-order Balmer lines compared to $\mathrm{H} \alpha$. The result is a relatively flatter decrement. 


\section{Activity: Bandheads}

Molecular rotational and vibrational transitions imprint large bandheads on the observed spectra of low-mass stars. The strength of the TiO bandheads in the visible is often used as a spectral-type discriminant (Reid et al., 1995a). Additionally, CaOH, $\mathrm{TiO}$ and $\mathrm{CaH}$ bandheads have been employed as temperature and metallicity indicators (Gizis, 1997; Hawley et al., 1999; Woolf \& Wallerstein, 2006). Following previous conventions (Reid et al., 1995a; Kirkpatrick et al., 1999), we provide measurements

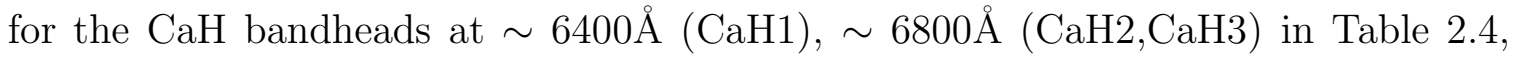
and for the $\mathrm{TiO}$ bandheads at $\sim 7050 \AA$ (TiO2, TiO4, TiO5) and $\sim 8430 \AA(\mathrm{TiO} 8)$ in Table 2.5. Note that $\mathrm{TiO} 2$ and $\mathrm{TiO} 4$ are sub-bands of the full TiO5 bandhead.

As first observed by Hawley et al. (1996), activity can introduce changes in the TiO bandheads (Hawley et al., 1999; Martín, 1999). Shown in Figure 2.8 is the TiO2 index as a function of the $\mathrm{TiO} 4$ index. For active stars, the strength of the TiO2 bandhead is increased (smaller index) at a given value of the TiO4 index. Alternately, at a given index of $\mathrm{TiO} 2$, the $\mathrm{TiO} 4$ index is weaker in active stars. This provides interesting constraints on the structure of the atmosphere, suggesting that the formation of $\mathrm{TiO}$, thought to take place near the temperature minimum region below the chromospheric temperature rise (Chabrier et al., 2005; Reid \& Hawley, 2005), is affected by the presence of an overlying chromosphere. The opposite behavior of these two subbands serves to decrease the sensitivity of TiO5 to chromospheric activity, making it a good temperature (spectral-type) proxy regardless of the activity level of the star (see Hawley et al., 1999).

\section{Activity: Spectral Features}

Two main effects influence the colors of active stars: the presence of emission lines

and changes in the continuum emission. To investigate these effects, we divided the active templates by their inactive counterparts. Figure 2.9 is an illustrative exam- 


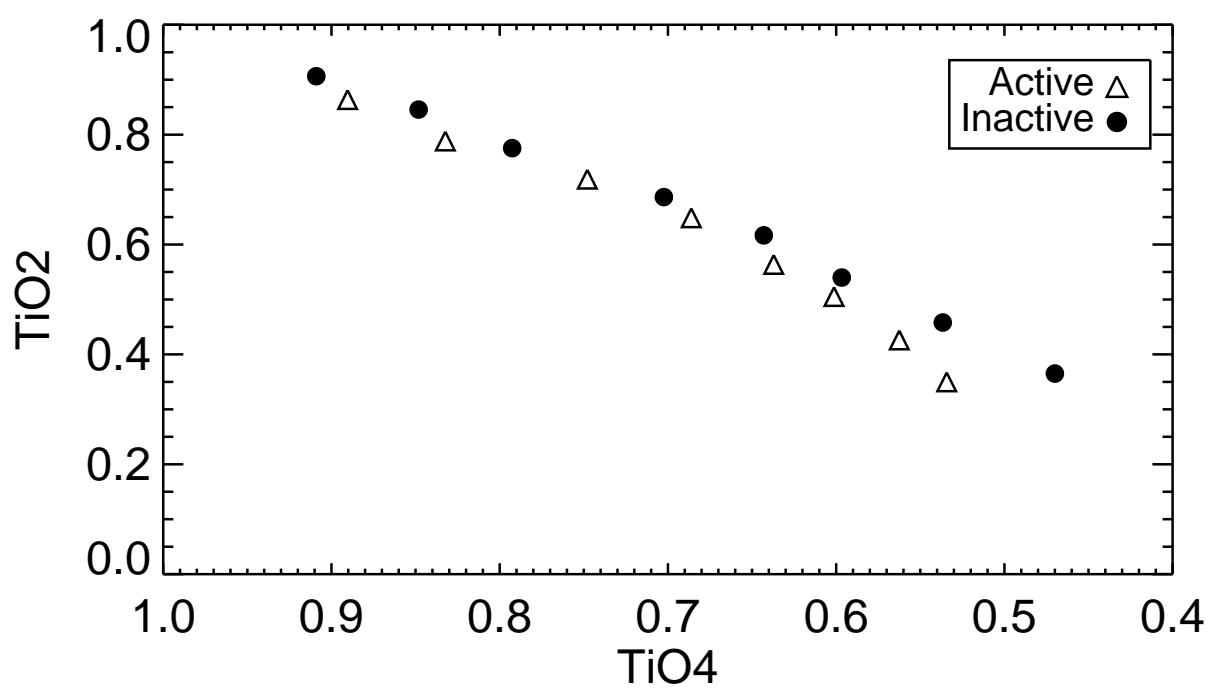

Figure $2.8 \mathrm{TiO} 2$ vs. $\mathrm{TiO} 4$ for active (open triangles) and inactive (filled circles) templates. At a constant $\mathrm{TiO} 4$ value, $\mathrm{TiO} 2$ is deeper (smaller index values) in the active stars, confirming the result originally discussed in Hawley et al. (1996).

ple of our analysis, showing the individual active and inactive M0 template spectra together with the ratio of the active to inactive flux. The approximate wavelength bounds of the SDSS $g, r$ and $i$ filters are indicated. The ratio shows enhanced blue continuum emission in the active template, and significantly enhanced emission lines, particularly in Ca II H and K. These effects lead to a bluer $(g-r)$ color for the active star (see Table 2.6, discussed further in $§ 2.5 .3$ below). The change in color is dominated by the continuum enhancement, with the increased emission line flux providing only a marginal effect. Similar continuum and line flux enhancements are observed during flares, suggesting that the active M0 template may include one or more stellar spectra obtained during low level flaring conditions. As described in Güdel et al. (2002), low level flaring maybe responsible for a significant fraction of the "quiescent" chromospheric emission observed on active stars.

The ratio also shows two "emission" lines in the $r$ band corresponding to emission in the core of the Na I D doublet $(\lambda \sim 5900 \AA$, doublet marginally resolved at SDSS 
resolution, but well resolved in our templates) and in $\mathrm{H} \alpha$. Again, these lines do not significantly contribute to the combined flux of the template in the $r$ band, as shown by the marginally redder $r-i$ color for the active template in Table 2.6. The spectral flux ratio in the $i$ band is very close to unity, with no strongly varying emission or continuum features between the active and inactive templates. This analysis suggests that changes in the continuum emission of active stars provide the most important contribution to observed color differences.

The variable strength of the $\mathrm{CaOH}(6230 \AA)$ bandhead is also of note. Shown in Figure 2.10 are the active to inactive ratios for M4-M7 subtypes. The growth of this feature indicates there is some dependence of the formation mechanism of $\mathrm{CaOH}$ on spectral type (effective temperature, mass), perhaps changing the position of the temperature minimum within the atmosphere. Note that the feature near this wavelength previously discussed as a good temperature indicator by Hawley et al. (1999) is actually due to $\mathrm{TiO}$ in early $\mathrm{M}$ dwarfs. $\mathrm{CaOH}$ begins to dominate the opacity in this region only at types later than M4, which were not available to observation in the clusters described by Hawley et al. (1999). Therefore these new SDSS observations are the first evidence of a real effect in the $\mathrm{CaOH}$ band that differs with the presence of a chromosphere and changes with spectral type. These observations, together with the differences in the $\mathrm{TiO} 2$ and $\mathrm{TiO} 4$ bands should provide strong constraints on the next generation of atmospheric models (including chromospheres) for M dwarfs.

\section{Metallicity: Spectral Features}

We explored the effects of metallicity on the spectra by comparing our composite templates to a low-metallicity subdwarf $([\mathrm{Fe} / \mathrm{H}] \sim-0.5$; Woolf \& Wallerstein, 2006) and a metal-rich Hyades dwarf $([\mathrm{Fe} / \mathrm{H}]=0.13$, Paulson et al., 2003), both observed with SDSS. The results are shown in Figure 2.11. Previous studies (West et al., 2004) indicate that subdwarfs are $\sim 0.2$ mags redder than solar-metallicity stars in $g-r$. This is most likely due to the multiple hydride bands present in the $g$ filter (Hartwick, 


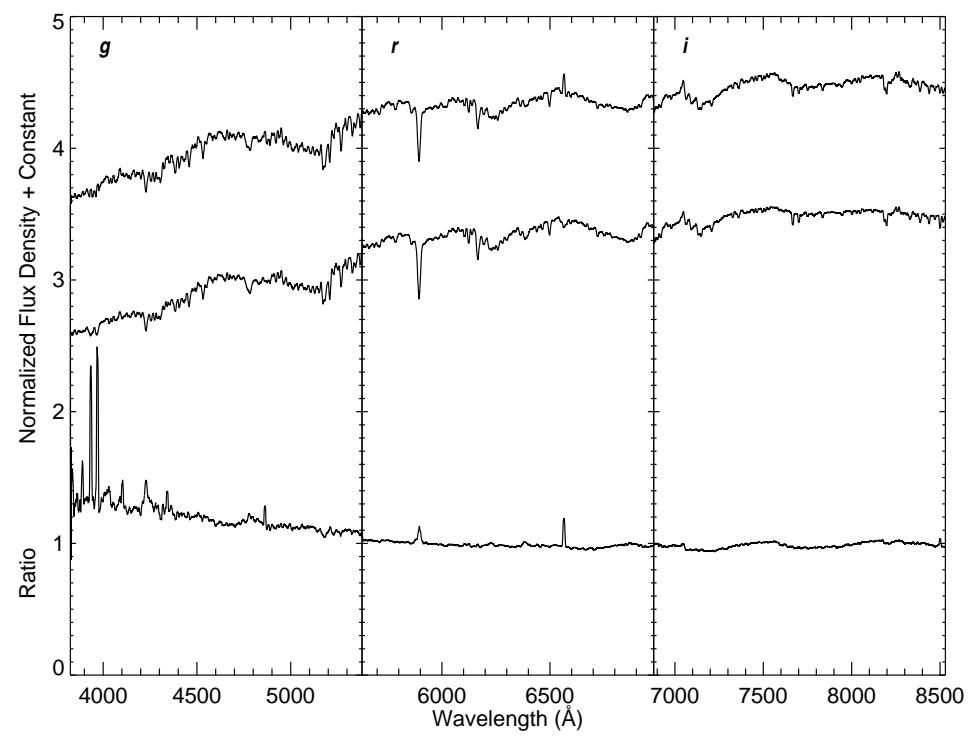

Figure 2.9 Shown is an illustrative example of our flux-ratio analysis. The active M0 spectral template (top) is divided by the inactive (middle) template. The resulting flux ratio is plotted on the bottom. The windows display the approximate wavelength bounds of the SDSS $g, r$, and $i$ filters.

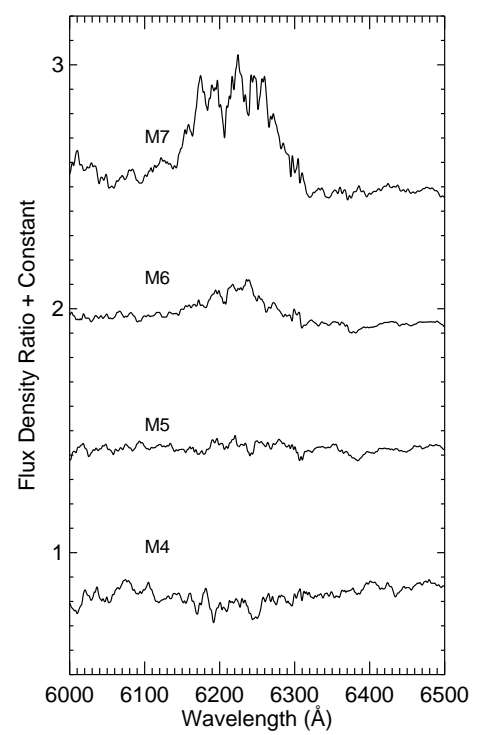

Figure 2.10 The ratio of the active to inactive flux for the M4-M7 templates is shown in the vicinity of the $\mathrm{CaOH}(6230 \AA)$ bandhead. Note the increase in the ratio at later types, indicating a shallower $\mathrm{CaOH}$ band in the active template compared to its inactive counterpart. Apparently, the formation mechanism of $\mathrm{CaOH}$ depends on both the star's spectral type (effective temperature) and the presence of a chromosphere. 


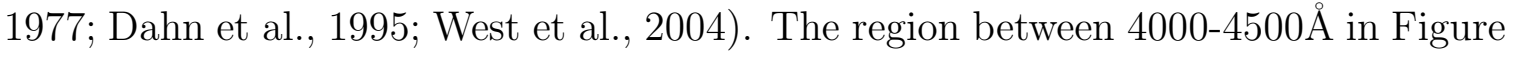
2.11 (left panel) shows that the flux in the subdwarf is depressed by $\sim 60 \%$ compared to the level present in the composite template. Strong hydride bands, such as $\mathrm{MgH}$ near $5000 \AA$ and $\mathrm{CaH}$ bands near $6800 \AA$ are also depressed. These bands are labeled in Figure 2.4.

In contrast, the metal-rich Hyades star (right panel of Fig. 2.11) shows mostly enhanced but variable continuum in the $g$ band, which is difficult to attribute to any particular feature. There is enhanced emission in the core of $\mathrm{Na} \mathrm{I} \mathrm{D} \mathrm{and} \mathrm{Ca} \mathrm{II} \mathrm{H} \mathrm{and}$ $\mathrm{K}$, but these are likely not strong enough to influence the colors. Unfortunately we do not have colors in the SDSS filters, measured with the 2.5m SDSS telescope, for the Hyades stars, and therefore cannot directly compare the spectral features with measured color differences between the metal-rich stars and our templates.

\subsubsection{Photometric Differences: Colors}

Photometry was obtained from the SDSS CAS for each star used in constructing the templates. The average colors for each template are listed in Table 2.6 by spectral type. Previous studies have been inconclusive, suggesting that active stars are marginally bluer in $U-B$ (Amado \& Byrne, 1997), redder in $V-I$ (Hawley et al., 1999) or not statistically different from inactive populations for SDSS colors (West et al., 2004). We computed the color difference for each spectral subtype (active color - inactive color) and averaged over spectral type. The data in Table 2.6 indicate the following general trends: active stars are $\sim 0.09 \pm 0.24$ mags bluer than their non-active counterparts in $u-g$, while they are $\sim 0.05 \pm 0.03$ mags redder in $i-z$. We note that while these trends are suggestive, but are within the scatter. No strong trends were present in $g-r$ or $r-i$.

Our goal was to link changes in the spectral features to differences in photometric colors. Due to the spectral coverage of the SDSS spectra, we are only able to investigate the $g-r$ and $r-i$ colors in detail, which did not demonstrate any dis- 

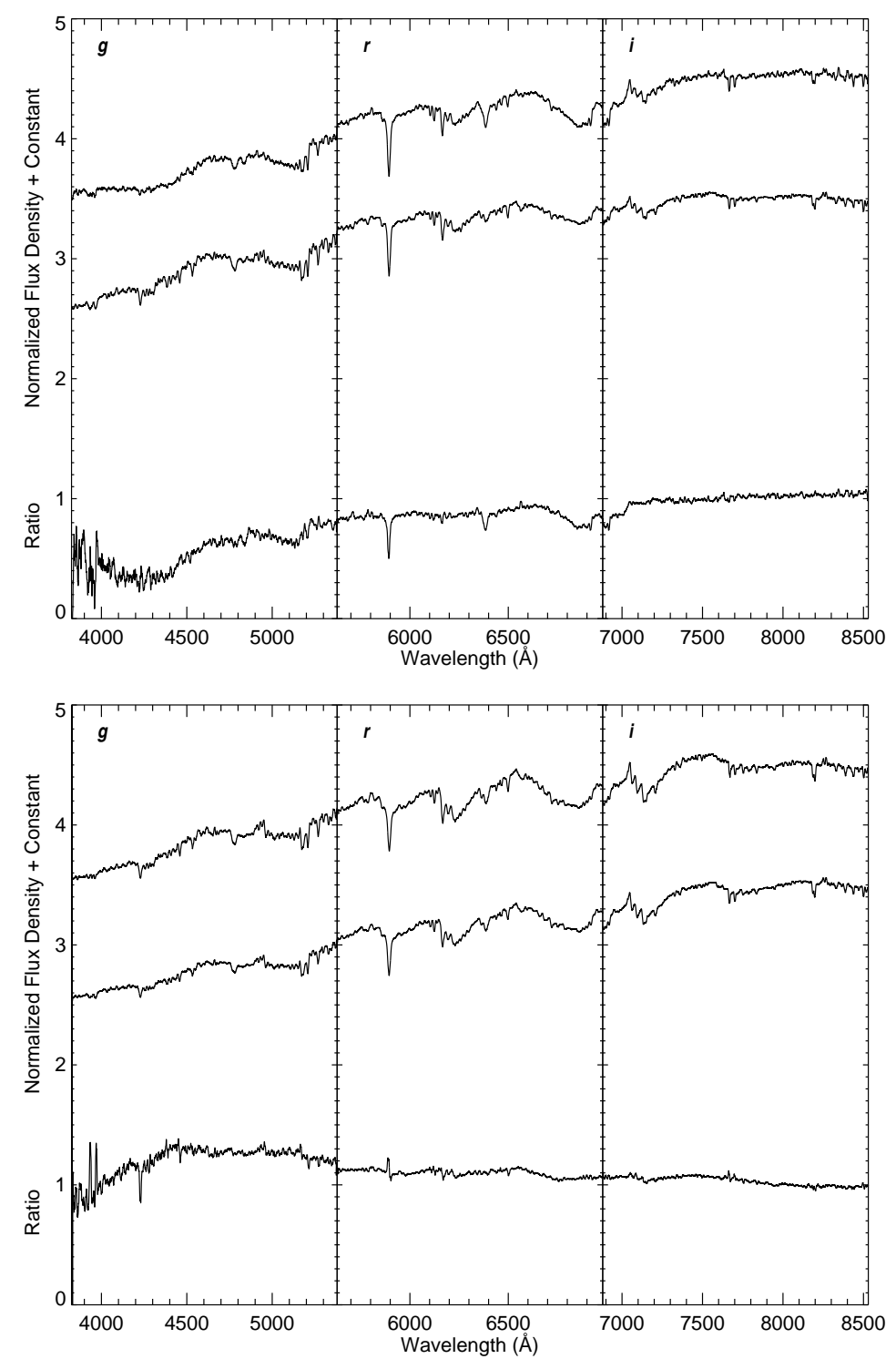

Figure 2.11 Shown are illustrative examples of our flux ratio analysis of an M0 subdwarf (left panel) and an M1 Hyades star (right panel). The subdwarf and Hyades star are the top spectra in their respective panels; the composite template of the same spectral type (from Figure 2.3) appears as the middle spectrum, and the ratio of the two spectra is shown on the bottom. 
cernible trends with activity. The bluer $g-r$ color in the M0 active template appears to be anomalous, and from the spectral analysis, may be due to low level flaring see $§ 2.5 .2$. This may also be simply due to the small number of spectra associated with the active M0 template. However, the photometric trend in $u-g$, where active stars were an average of $\sim 0.09$ mags bluer than inactive stars, may be reflecting the presence of similar low level flaring in many of the active templates, as the enhanced blue continuum during flares will appear even more strongly in the $u$ band (Moffett \& Bopp, 1976; Hawley \& Pettersen, 1991).

The small number of M subdwarfs identified in the SDSS database, and the lack of SDSS photometry for the Hyades M dwarfs prevented us from investigating color differences due to metallicity. As noted in the previous section, West et al. (2004) showed that $\mathrm{M}$ subdwarfs are $\sim 0.2$ mags redder in $g-r$ than their solar-metallicity counterparts.

\subsection{Conclusions}

We used the large SDSS spectral database from DR3 to form active, inactive and composite template spectra of $\mathrm{M}$ dwarfs spanning types M0-L0, on a uniform, zerovelocity scale. Our spectral templates provide suitable radial velocity standards for

analyzing spectra with $\mathrm{R} \sim 1,800$, with an external accuracy of $3.8 \mathrm{~km} \mathrm{~s}^{-1}$, within the quoted error associated with the wavelength scale for SDSS spectroscopy (York et al., 2000). Internally, the templates are consistent to $<1 \mathrm{~km} \mathrm{~s}^{-1}$.

The magnetically active templates, as identified by the presence of $\mathrm{H} \alpha$ emission in the individual stellar spectra, showed little difference in the measured Balmer decrements with spectral type, indicating that chromospheric structure and heating are apparently similar through the $\mathrm{M}$ dwarf sequence. Flares cause much larger changes in the decrement. We found some evidence that color changes (active stars appearing bluer in $u-g$ and in one case in $g-r$ ) are due primarily to blue continuum enhancements in the active stars, which may be due to intermittent low-level flaring. 
In general, chromospheric line emission has a negligible effect on the colors of active stars. Molecular bands including $\mathrm{TiO} 2, \mathrm{TiO} 4$ and $\mathrm{CaOH}$ showed significant changes between the active and inactive templates.

With regard to metallicity, our findings extend the earlier study by West et al. (2004), which found subdwarfs to be $\sim 0.2$ mags redder in $g-r$. Our spectral analysis shows that the flux in the blue is depressed by as much as $60 \%$, and that the strong $\mathrm{MgH}$ and $\mathrm{CaH}$ bands are significantly deeper in the subdwarfs. The spectral analysis of metal-rich Hyades stars $([\mathrm{Fe} / \mathrm{H}]=0.13$, Paulson et al., 2003) showed continuum differences, but these were not obviously attributed to any particular features.

The authors would like to thank Andrew Becker and Kelle Cruz for their enlightening conversations. The authors gratefully acknowledge the support of NSF grant AST02-05875 and NASA ADP grant NAG5-13111. This research has made use of NASA's Astrophysics Data System Abstract Service, the SIMBAD database, operated at CDS, Strasbourg, France. This project made extensive use of SDSS data. Funding for the SDSS and SDSS-II has been provided by the Alfred P. Sloan Foundation, the Participating Institutions, the National Science Foundation, the U.S. Department of Energy, the National Aeronautics and Space Administration, the Japanese Monbukagakusho, the Max Planck Society, and the Higher Education Funding Council for England. The SDSS Web Site is http://www.sdss.org/.

The SDSS is managed by the Astrophysical Research Consortium for the Participating Institutions. The Participating Institutions are the American Museum of Natural History, Astrophysical Institute Potsdam, University of Basel, Cambridge University, Case Western Reserve University, University of Chicago, Drexel University, Fermilab, the Institute for Advanced Study, the Japan Participation Group, Johns Hopkins University, the Joint Institute for Nuclear Astrophysics, the Kavli Institute for Particle Astrophysics and Cosmology, the Korean Scientist Group, the Chinese Academy of Sciences (LAMOST), Los Alamos National Laboratory, the Max-PlanckInstitute for Astronomy (MPIA), the Max-Planck-Institute for Astrophysics (MPA), 
Table 2.1. DAS Query Color Ranges

\begin{tabular}{ccc}
\hline \hline Sp. Type & $r-i$ & $i-z$ \\
\hline M0 & $0.50-0.85$ & $0.30-0.50$ \\
M1 & $0.60-1.15$ & $0.30-0.65$ \\
M2 & $0.80-1.30$ & $0.40-0.75$ \\
M3 & $0.90-1.50$ & $0.40-0.90$ \\
M4 & $1.10-1.80$ & $0.60-1.10$ \\
M5 & $1.45-2.20$ & $0.80-1.15$ \\
M6 & $1.65-2.25$ & $0.90-1.20$ \\
M7 & $1.90-2.70$ & $0.95-1.65$ \\
M8 & $2.65-2.85$ & $1.20-1.90$ \\
M9 & $2.85-3.05$ & $1.25-1.80$ \\
L0 & $2.30-2.70$ & $1.70-1.90$ \\
\hline
\end{tabular}

New Mexico State University, Ohio State University, University of Pittsburgh, University of Portsmouth, Princeton University, the United States Naval Observatory, and the University of Washington. 
Table 2.2. Active Template Decrements

\begin{tabular}{|c|c|c|c|c|c|c|}
\hline Sp. Type & $\mathrm{H} \alpha$ & $\mathrm{H} \beta$ & $\mathrm{H} \gamma$ & $\mathrm{H} \delta$ & $\mathrm{H} 8$ & Ca II K \\
\hline M0 & $2.09(0.22)$ & $1.00(0.15)$ & $\cdots$ & $\cdots$ & $\cdots$ & $\cdots$ \\
\hline M1 & $2.33(0.50)$ & $1.00(0.28)$ & $\cdots$ & $\cdots$ & $\cdots$ & $0.19(0.16)$ \\
\hline M2 & $\cdots$ & $\cdots$ & $\cdots$ & $\cdots$ & $\cdots$ & $\cdots$ \\
\hline M3 & $3.08(0.73)$ & $1.00(0.28)$ & $\cdots$ & $\cdots$ & $\ldots$ & $\cdots$ \\
\hline M4 & $3.37(0.67)$ & $1.00(0.26)$ & $0.51(0.26)$ & $0.43(0.25)$ & $\cdots$ & $\cdots$ \\
\hline M5 & $4.27(1.33)$ & $1.00(0.34)$ & $1.12(0.41)$ & $0.52(0.30)$ & $0.13(0.36)$ & $0.86(0.38)$ \\
\hline M6 & $3.68(0.66)$ & $1.00(0.24)$ & $0.76(0.25)$ & $0.36(0.22)$ & $0.31(0.33)$ & $0.67(0.27)$ \\
\hline M7 & $4.18(0.71)$ & $1.00(0.23)$ & $0.72(0.23)$ & $0.47(0.23)$ & $0.25(0.32)$ & $0.80(0.27)$ \\
\hline M8 & $5.90(1.11)$ & $1.00(0.25)$ & $0.90(0.28)$ & $0.64(0.28)$ & $0.32(0.39)$ & $\ldots$ \\
\hline M9 & $\cdots$ & $\cdots$ & $\cdots$ & $\cdots$ & $\cdots$ & $\cdots$ \\
\hline L0 & $\cdots$ & $\cdots$ & $\cdots$ & $\cdots$ & $\cdots$ & $\cdots$ \\
\hline Average $^{a}$ & $3.61(1.21)$ & $1.00(0.00)$ & $0.80(0.23)$ & $0.48(0.11)$ & $0.25(0.09)$ & $0.78(0.10)$ \\
\hline AD Leo (Hawley \& Pettersen, 1991) & $\ldots$ & 1.00 & 0.81 & 0.69 & 0.50 & 0.18 \\
\hline Quiet Model (Allred et al., 2006) & 2.20 & 1.00 & 0.58 & 0.21 & 0.34 & 3.65 \\
\hline Flare Model (Allred et al., 2006) & 0.57 & 1.00 & 0.84 & 0.75 & 0.54 & 0.10 \\
\hline
\end{tabular}

Note. - Decrement measurements are reported with measurement errors in parentheses.

${ }^{a}$ Errors reported on means are $1 \sigma$ of individual decrement measurements.

Table 2.3. Active Template Equivalent Widths and $L_{\mathrm{H} \alpha} / L_{\mathrm{bol}, i-z}$

\begin{tabular}{|c|c|c|c|c|c|c|c|}
\hline Sp. Type & $\mathrm{H} \alpha \mathrm{EW}$ & $\mathrm{H} \beta \mathrm{EW}$ & $\mathrm{H} \gamma \mathrm{EW}$ & $\mathrm{H} \delta \mathrm{EW}$ & H8 EW & Ca II K EW & $L_{\mathrm{H} \alpha} / L_{\mathrm{bol}, i-z}$ \\
\hline M0 & $1.39(0.04)$ & $1.09(0.11)$ & $\ldots$ & $\ldots$ & $\ldots$ & $\ldots$ & $2.15 \mathrm{E}-04(5.48 \mathrm{E}-05)$ \\
\hline M1 & $1.33(0.10)$ & $1.54(0.31)$ & $\ldots$ & $\ldots$ & $\ldots$ & $1.03(0.84)$ & $1.28 \mathrm{E}-04(5.83 \mathrm{E}-05)$ \\
\hline M2 & $3.57(0.10)$ & - 190 & $\ldots$ & $\ldots$ & $\ldots$ & $\ldots$ & $3.24 \mathrm{E}-04(2.87 \mathrm{E}-05)$ \\
\hline M3 & $2.45(0.31)$ & $2.40(0.48)$ & $\ldots$ & $\ldots$ & $\ldots$ & $\ldots$ & $1.42 \mathrm{E}-04(7.00 \mathrm{E}-05)$ \\
\hline M4 & $4.12(0.35)$ & $5.10(0.93)$ & $6.33(3.09)$ & $7.17(4.09)$ & $\ldots$ & $\ldots$ & $1.75 \mathrm{E}-04(6.08 \mathrm{E}-05)$ \\
\hline M5 & $5.85(1.16)$ & $5.56(1.34)$ & $16.15(4.56)$ & $8.28(4.41)$ & $3.78(10.26)$ & $25.09(12.75)$ & $1.82 \mathrm{E}-04(7.14 \mathrm{E}-05)$ \\
\hline M6 & $6.06(0.39)$ & $7.94(1.34)$ & $18.88(5.55)$ & $9.36(5.75)$ & $9.26(10.20)$ & $19.10(9.16)$ & $1.35 \mathrm{E}-04(2.43 \mathrm{E}-05)$ \\
\hline M7 & $8.15(0.50)$ & $10.47(1.70)$ & $25.85(7.89)$ & $15.73(7.64)$ & $10.29(13.44)$ & $21.30(8.84)$ & $1.01 \mathrm{E}-04(3.45 \mathrm{E}-05)$ \\
\hline M8 & $10.99(0.64)$ & $12.23(2.24)$ & $113.05(60.46)$ & $38.65(19.74)$ & $20.34(27.37)$ & $\ldots$ & $4.41 \mathrm{E}-05(1.14 \mathrm{E}-05)$ \\
\hline M9 & $6.10(0.18)$ & $\ldots$ & $\ldots$ & $\ldots$ & $10.94(14.52)$ & $\ldots$ & $2.52 \mathrm{E}-05(6.41 \mathrm{E}-06)$ \\
\hline L0 & $6.86(0.41)$ & $\ldots$ & $\ldots$ & $\ldots$ & $\ldots$ & $\ldots$ & $1.97 \mathrm{E}-05(2.50 \mathrm{E}-06)$ \\
\hline
\end{tabular}

Note. - Equivalent Widths are reported in Åwith measurement errors in parentheses.

$L_{\mathrm{H} \alpha} / L_{\mathrm{bol}, i-z}$ measurement errors are also in parentheses. 
Table 2.4. Template Bandheads

\begin{tabular}{|c|c|c|c|c|c|c|c|c|c|}
\hline Sp. Type & & CaH1 & & & $\mathrm{CaH} 2$ & & & $\mathrm{CaH} 3$ & \\
\hline & Active & Inactive & All & Active & Inactive & All & Active & Inactive & All \\
\hline M0 & $0.94(0.00)$ & $0.91(0.01)$ & $0.91(0.01)$ & $0.79(0.00)$ & $0.79(0.01)$ & $0.79(0.01)$ & $0.87(0.00)$ & $0.89(0.01)$ & $0.89(0.01)$ \\
\hline M1 & $0.86(0.01)$ & $0.85(0.02)$ & $0.85(0.02)$ & $0.68(0.01)$ & $0.67(0.01)$ & $0.67(0.01)$ & $0.82(0.01)$ & $0.83(0.02)$ & $0.83(0.02)$ \\
\hline M2 & $0.81(0.01)$ & $0.80(0.03)$ & $0.80(0.03)$ & $0.49(0.01)$ & $0.56(0.03)$ & $0.56(0.03)$ & $0.68(0.01)$ & $0.76(0.04)$ & $0.76(0.04)$ \\
\hline M3 & $0.74(0.04)$ & $0.78(0.07)$ & $0.78(0.07)$ & $0.48(0.03)$ & $0.48(0.06)$ & $0.48(0.06)$ & $0.71(0.04)$ & $0.72(0.09)$ & $0.72(0.09)$ \\
\hline M4 & $0.76(0.03)$ & $0.76(0.04)$ & $0.76(0.04)$ & $0.39(0.02)$ & $0.43(0.02)$ & $0.42(0.02)$ & $0.65(0.03)$ & $0.69(0.04)$ & $0.68(0.04)$ \\
\hline M5 & $0.75(0.04)$ & $0.80(0.03)$ & $0.78(0.04)$ & $0.37(0.03)$ & $0.38(0.02)$ & $0.38(0.02)$ & $0.64(0.04)$ & $0.67(0.03)$ & $0.66(0.04)$ \\
\hline M6 & $0.76(0.03)$ & $0.79(0.07)$ & $0.77(0.05)$ & $0.33(0.01)$ & $0.33(0.03)$ & $0.33(0.02)$ & $0.60(0.02)$ & $0.64(0.06)$ & $0.62(0.04)$ \\
\hline M7 & $0.78(0.04)$ & $0.77(0.04)$ & $0.78(0.04)$ & $0.29(0.01)$ & $0.28(0.01)$ & $0.28(0.01)$ & $0.58(0.02)$ & $0.59(0.02)$ & $0.58(0.02)$ \\
\hline M8 & $0.84(0.04)$ & $\ldots$ & $0.85(0.04)$ & $0.28(0.01)$ & $\ldots$ & $0.28(0.01)$ & $0.57(0.01)$ & $\ldots$ & $0.57(0.01)$ \\
\hline M9 & $0.90(0.03)$ & $\ldots$ & $0.91(0.03)$ & $0.30(0.01)$ & $\ldots$ & $0.30(0.01)$ & $0.63(0.01)$ & $\ldots$ & $0.63(0.01)$ \\
\hline L0 & $0.97(0.04)$ & $\ldots$ & $0.96(0.04)$ & $0.50(0.01)$ & $\ldots$ & $0.50(0.01)$ & $0.71(0.01)$ & $\ldots$ & $0.71(0.01)$ \\
\hline
\end{tabular}

Note. - Measurement errors are given in parentheses. 
Table 2.5. Template Bandheads

\begin{tabular}{|c|c|c|c|c|c|c|c|c|c|c|c|c|}
\hline Sp. Type & & $\mathrm{TiO} 2$ & & & TiO4 & & & TiO5 & & & TiO8 & \\
\hline & Active & Inactive & All & Active & Inactive & All & Active & Inactive & All & Active & Inactive & All \\
\hline M0 & $0.86(0.01)$ & $0.91(0.02)$ & $0.91(0.02)$ & $0.89(0.01)$ & $0.91(0.02)$ & $0.91(0.02)$ & $0.78(0.01)$ & $0.82(0.01)$ & $0.82(0.01)$ & $0.97(0.00)$ & $0.99(0.01)$ & $0.99(0.01)$ \\
\hline M1 & $0.79(0.03)$ & $0.85(0.03)$ & $0.84(0.03)$ & $0.83(0.02)$ & $0.85(0.02)$ & $0.85(0.02)$ & $0.71(0.01)$ & $0.72(0.02)$ & $0.72(0.02)$ & $0.98(0.01)$ & $0.98(0.01)$ & $0.98(0.01)$ \\
\hline M2 & $0.72(0.02)$ & $0.78(0.05)$ & $0.77(0.05)$ & $0.75(0.02)$ & $0.79(0.05)$ & $0.79(0.05)$ & $0.52(0.01)$ & $0.61(0.03)$ & $0.60(0.03)$ & $0.97(0.01)$ & $0.97(0.03)$ & $0.97(0.03)$ \\
\hline M3 & $0.65(0.07)$ & $0.69(0.13)$ & $0.69(0.13)$ & $0.69(0.06)$ & $0.70(0.10)$ & $0.70(0.10)$ & $0.49(0.03)$ & $0.49(0.07)$ & $0.49(0.07)$ & $0.99(0.03)$ & $0.92(0.06)$ & $0.93(0.06)$ \\
\hline M4 & $0.56(0.04)$ & $0.62(0.05)$ & $0.61(0.05)$ & $0.64(0.04)$ & $0.64(0.04)$ & $0.64(0.04)$ & $0.39(0.02)$ & $0.41(0.03)$ & $0.41(0.02)$ & $0.91(0.02)$ & $0.90(0.03)$ & $0.90(0.02)$ \\
\hline M5 & $0.51(0.05)$ & $0.54(0.04)$ & $0.53(0.05)$ & $0.60(0.04)$ & $0.60(0.04)$ & $0.60(0.04)$ & $0.34(0.03)$ & $0.34(0.02)$ & $0.34(0.02)$ & $0.84(0.03)$ & $0.84(0.02)$ & $0.84(0.03)$ \\
\hline M6 & $0.43(0.02)$ & $0.46(0.07)$ & $0.44(0.05)$ & $0.56(0.03)$ & $0.54(0.07)$ & $0.55(0.05)$ & $0.28(0.01)$ & $0.27(0.03)$ & $0.27(0.02)$ & $0.77(0.01)$ & $0.77(0.04)$ & $0.77(0.03)$ \\
\hline M7 & $0.35(0.02)$ & $0.37(0.02)$ & $0.35(0.02)$ & $0.53(0.03)$ & $0.47(0.03)$ & $0.51(0.03)$ & $0.22(0.01)$ & $0.20(0.01)$ & $0.22(0.01)$ & $0.68(0.01)$ & $0.68(0.01)$ & $0.68(0.01)$ \\
\hline M8 & $0.32(0.02)$ & $\ldots$ & $0.32(0.02)$ & $0.65(0.03)$ & $\ldots$ & $0.65(0.03)$ & $0.25(0.01)$ & $\ldots$ & $0.25(0.01)$ & $0.55(0.01)$ & $\ldots$ & $0.55(0.01)$ \\
\hline M9 & $0.32(0.01)$ & $\ldots$ & $0.32(0.01)$ & $0.62(0.02)$ & $\ldots$ & $0.61(0.02)$ & $0.26(0.01)$ & $\ldots$ & $0.26(0.01)$ & $0.54(0.00)$ & $\ldots$ & $0.54(0.00)$ \\
\hline L0 & $0.65(0.04)$ & $\cdots$ & $0.64(0.04)$ & $0.87(0.05)$ & $\cdots$ & $0.86(0.05)$ & $0.64(0.02)$ & $\cdots$ & $0.64(0.02)$ & $0.62(0.01)$ & $\cdots$ & $0.62(0.01)$ \\
\hline
\end{tabular}

Note. - Measurement errors are given in parentheses. 
Table 2.6. Template Colors

\begin{tabular}{|c|c|c|c|c|c|c|c|c|c|c|c|c|c|c|c|}
\hline \multirow{3}{*}{$\begin{array}{c}\text { Sp. Type } \\
\text { M0 }\end{array}$} & \multicolumn{3}{|c|}{ Num. Stars ${ }^{a}$} & \multicolumn{3}{|c|}{$u-g$} & \multicolumn{3}{|c|}{$g-r$} & \multicolumn{3}{|c|}{$r-i$} & \multicolumn{3}{|c|}{$i-z$} \\
\hline & & & & Active & Inactive & All & Active & Inactive & All & Active & Inactive & All & Active & Inactive & All \\
\hline & 7 & 570 & 577 & $2.21(0.40)$ & $2.56(0.66)$ & $2.56(0.66)$ & $1.24(0.38)$ & $1.40(0.55)$ & $1.40(0.54)$ & $0.68(0.13)$ & $0.65(0.12)$ & $0.65(0.12)$ & $0.41(0.09)$ & $0.39(0.08)$ & $0.39(0.08)$ \\
\hline M1 & 4 & 275 & 279 & $2.17(0.51)$ & $2.54(0.64)$ & $2.53(0.64)$ & $1.43(0.19)$ & $1.47(0.44)$ & $1.47(0.44)$ & $0.77(0.16)$ & $0.80(0.11)$ & $0.80(0.11)$ & $0.58(0.16)$ & $0.47(0.07)$ & $0.47(0.07)$ \\
\hline M2 & 1 & 66 & 67 & $2.69(0.03)$ & $2.29(0.80)$ & $2.30(0.80)$ & $1.90(0.03)$ & $1.60(0.36)$ & $1.60(0.35)$ & $1.07(0.03)$ & $1.04(0.18)$ & $1.04(0.18)$ & $0.60(0.03)$ & $0.59(0.11)$ & $0.59(0.11)$ \\
\hline M3 & 6 & 186 & 192 & $2.00(0.35)$ & $2.18(0.85)$ & $2.18(0.84)$ & $1.69(0.18)$ & $1.59(0.23)$ & $1.60(0.22)$ & $1.30(0.15)$ & $1.28(0.19)$ & $1.28(0.19)$ & $0.76(0.17)$ & $0.70(0.12)$ & $0.70(0.12)$ \\
\hline M4 & 25 & 137 & 162 & $2.28(1.09)$ & $2.28(0.77)$ & $2.28(0.82)$ & $1.60(0.17)$ & $1.55(0.21)$ & $1.56(0.20)$ & $1.49(0.28)$ & $1.42(0.16)$ & $1.43(0.19)$ & $0.87(0.12)$ & $0.79(0.10)$ & $0.81(0.10)$ \\
\hline M5 & 171 & 235 & 406 & $2.13(0.95)$ & $2.18(0.89)$ & $2.16(0.92)$ & $1.52(0.30)$ & $1.57(0.24)$ & $1.55(0.27)$ & $1.74(0.21)$ & $1.72(0.21)$ & $1.73(0.21)$ & $0.98(0.12)$ & $0.95(0.11)$ & $0.96(0.12)$ \\
\hline M6 & 1132 & 899 & 2031 & $2.12(0.90)$ & $2.17(0.93)$ & $2.15(0.91)$ & $1.59(0.13)$ & $1.55(0.13)$ & $1.57(0.13)$ & $1.98(0.11)$ & $1.99(0.12)$ & $1.98(0.12)$ & $1.10(0.06)$ & $1.09(0.06)$ & $1.09(0.06)$ \\
\hline M7 & 400 & 150 & 550 & $1.89(0.91)$ & $1.98(0.93)$ & $1.92(0.92)$ & $1.63(0.17)$ & $1.55(0.15)$ & $1.61(0.17)$ & $2.33(0.19)$ & $2.35(0.18)$ & $2.34(0.19)$ & $1.31(0.12)$ & $1.26(0.09)$ & $1.29(0.11)$ \\
\hline M8 & 16 & $\ldots$ & 16 & $1.65(0.93)$ & $\ldots$ & $1.65(0.93)$ & $1.80(0.16)$ & $\ldots$ & $1.80(0.16)$ & $2.76(0.12)$ & $\ldots$ & $2.76(0.12)$ & $1.71(0.09)$ & $\ldots$ & $1.71(0.09)$ \\
\hline M9 & 5 & $\ldots$ & 5 & $1.79(0.79)$ & $\ldots$ & $1.79(0.79)$ & $1.74(0.14)$ & $\ldots$ & $1.74(0.14)$ & $2.83(0.07)$ & $\ldots$ & $2.83(0.07)$ & $1.70(0.09)$ & $\ldots$ & $1.70(0.09)$ \\
\hline L0 & 4 & $\cdots$ & 4 & $1.35(1.35)$ & $\ldots$ & $1.35(1.35)$ & $2.50(0.39)$ & $\ldots$ & $2.50(0.39)$ & $2.54(0.08)$ & $\cdots$ & $2.54(0.08)$ & $1.83(0.04)$ & $\cdots$ & $1.83(0.04)$ \\
\hline
\end{tabular}

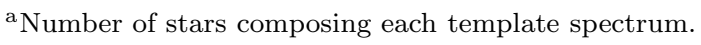

Note. - Mean SDSS colors are reported in magnitudes, with the one $\sigma$ spread at each spectral type reported in parentheses. 


\section{Chapter 3}

\section{EXPLORING THE LOCAL MILKY WAY: M DWARFS AS TRACERS OF GALACTIC POPULATIONS}

\subsection{Chapter Summary}

We have assembled a spectroscopic sample of low-mass dwarfs observed as part of the Sloan Digital Sky Survey along one Galactic sightline, designed to investigate the observable properties of the thin and thick disks. This sample of $\sim 7400 \mathrm{~K}$ and M stars also has measured ugriz photometry, proper motions, and radial velocities. We have computed $U V W$ space motion distributions, and investigate their structure with respect to vertical distance from the Galactic Plane. We place constraints on the velocity dispersions of the thin and thick disks, using two-component Gaussian fits. We also compare these kinematic distributions to a leading Galactic model. Finally, we investigate other possible observable differences between the thin and thick disks, such as color, active fraction and metallicity.

Sections 3.2 through 3.6 of this chapter was originally published in collaboration with Jeffrey A. Munn, Suzanne L. Hawley, Andrew A. West, Kevin R. Covey and Donald P. Schneider in the December 2007 edition of the Astronomical Journal (Bochanski et al., 2007a, ; AJ Vol. 134, pp. 2418; (c) 2007 by the American Astronomical Society) and is reproduced below with permission of the American Astronomical Society.

\subsection{Introduction}

Modeling the Galaxy presents a challenging breadth of problems to both theorists and observers. Large N-Body simulations employing the constraints of gravity and 
$\Lambda$ cold dark matter cosmology have sought to recreate the infant Galaxy, tracing the formation and collapse of baryons within the dark matter halo (Brook et al., 2004; Governato et al., 2007). Observationally, these simulations are constrained by rotational velocities and luminosity profiles of extragalactic systems (Dalcanton et al., 1997). Closer to home, observers seek to reconstruct the history of the Milky Way's cannibalistic mergers through photometric identification of tidal debris, such as the Sagittarius dwarf (Ibata et al., 1994). Spectroscopy is also employed to find surviving, co-moving stars of similar metallicity (e.g., Yanny et al., 2003).

A particularly interesting problem to both the theorist and observer is the formation and nature of the thick disk (Gilmore \& Reid, 1983). This population has been explored extensively, mainly through star counts (e.g. Gilmore \& Reid, 1983; Reid \& Majewski, 1993; Buser et al., 1999; Norris, 1999; Siegel et al., 2002). However, the thick disk scale height and local density normalization are still uncertain (Norris, 1999), with values of $h_{\text {thick }}$ ranging from $\sim 700-1500 \mathrm{pc}$ and local normalizations between $2 \%$ and $15 \%$. Larger scale heights are usually coupled to lower normalization values (see Figure 1 of Siegel et al., 2002). The scale length of the thick disk is also uncertain, though typically scale lengths larger than the thin disk are inferred (Chen et al., 2001; Larsen \& Humphreys, 2003). The thick disk is thought to be an older, metal-poor population (Reid \& Majewski, 1993; Chiba \& Beers, 2000). Metallicity differences, such as $\alpha$ element enhancement, have been explored (Bensby et al., 2003), but other observables, such as photometric color and chromospheric activity trends, have yet to be studied in detail. Thick disks observed in external galaxies (Yoachim \& Dalcanton, 2006) often appear to have structural parameters and kinematics similar to the Milky Way.

Modeling the observable properties of the thin and thick disks, namely star counts (Reid \& Majewski, 1993; Reid, 1993) and kinematics (Mendez \& van Altena, 1996; Robin et al., 2003; Vallenari et al., 2006), has undergone a resurgence in recent years. The seminal work of Bahcall \& Soneira (1980) laid the foundation for modeling star 
counts of the smooth Galactic components. Present-day models, such as the Besançon model (Robin et al., 2003), and the Padova model (Vallenari et al., 2006) also incorporate kinematics, allowing for robust comparisons to observations. It is important to rigorously test their predictions against actual observed spatial and kinematic distributions.

Low-mass dwarfs are both ubiquitous and long-lived (Laughlin et al., 1997), and serve as excellent tracers of the Galactic potential (Wilson \& Woolley, 1970; Wielen, 1977b; West et al., 2006). Modern surveys, such as the Sloan Digital Sky Survey (SDSS; York et al., 2000), are sensitive to $\mathrm{K}$ and early $\mathrm{M}$ dwarfs at distances of $\sim 1$ kpc above the Galactic Plane, probing the transition between the thin (Reid et al., 1997) and thick (Kerber et al., 2001) disks. At these distances, we expect about $20 \%$ of the observed stars to be thick disk members, assuming a local normalization of $2 \%$ (Reid \& Majewski, 1993) and scale heights of $300 \mathrm{pc}$ and $1400 \mathrm{pc}$ for the thin and thick disks, respectively. Star counts of low-mass dwarfs have been used to determine Galactic structural properties (Reid et al., 1997 and references therein). These studies sought to determine the vertical scale height and local normalization of the thin and thick disks (Siegel et al., 2002), as well as the underlying mass function of these populations (Martini \& Osmer, 1998; Phleps et al., 2000; Covey et al., 2008). The chemical evolution of the Galaxy has also been explored with red dwarfs (Reid et al., 1997), using molecular band indices as a proxy for metallicity (Gizis, 1997).

In addition to their utility as Galactic tracers, low-mass dwarfs have intrinsic properties, such as chromospheric activity (West et al., 2004; Bochanski et al., 2005; Schmidt et al., 2007), that can be placed in a Galactic context. Additionally, metallicity may be probed using subdwarfs (Lépine et al., 2003a), readily identified by their spectra, which show enhanced calcium hydride $(\mathrm{CaH})$ absorption. Subdwarfs have been easily detected in large-scale surveys such as the SDSS (West et al., 2004).

Kinematic studies of low-mass stars have a rich historical background. Samples are typically drawn from proper motion surveys, such as the New Luyten Two Tenths 
(NLTT) catalog (Luyten, 1979) and the Lowell Proper Motion Survey (Giclas et al., 1971). Efforts have been made to identify nearby stars in these surveys, for example by Gliese \& Jahreiss (1991). However, proper motion surveys possess inherent kinematic bias. The McCormick sample (Vyssotsky, 1956), assembled from 875 spectroscopically confirmed $\mathrm{K}$ and $\mathrm{M}$ dwarfs, has been frequently studied as a kinematically unbiased sample (Wielen, 1977b; Weis \& Upgren, 1995; Ratnatunga \& Upgren, 1997), although it has been suggested that the sample may be biased towards higher space motions (Reid et al., 1995a). The Palomar-Michigan State University survey (PMSU; Reid et al., 1995a; Hawley et al., 1996; Gizis et al., 2002; Reid et al., 2002) is among the largest prior spectroscopic surveys of low-mass stars, obtaining spectral types and radial velocities of $\sim 1700 \mathrm{M}$ dwarfs. The PMSU sample, which targeted objects from the Third Catalogue of Nearby Stars (Gliese \& Jahreiss, 1991), was used to construct a volume complete, kinematically unbiased catalog of $\sim 500$ stars, sampling distances to $\sim 25$ pc. Other surveys, such as the 100 pc survey (Bochanski et al., 2005), have also used low-mass stars as kinematic probes. In Table 3.1, we summarize the sample sizes and approximate distance limits of previous major kinematic surveys of low-mass dwarfs, along with the mean velocity dispersions for each study. It is clear that our sample of several thousand stars out to distances of $\sim 1 \mathrm{kpc}$ results in a study of Galactic kinematics using low-mass stars with unprecedented statistical significance.

In this paper, we present our examination of the properties of the thin and thick disks using a SDSS Low-Mass Spectroscopic Sample (SLoMaSS) of K and M dwarfs that is an order of magnitude larger than previous samples. In $\S 3.3$, we describe the SDSS spectroscopic and photometric observations that comprise SLoMaSS. The resulting distances and stellar velocities are presented in $\S 3.4$. In $\S 3.5$, we detail our efforts to separate the observed stars into two populations, search for kinematic, metallicity and color gradients and compare our results to a contemporary Galactic model. Finally, §3.6 summarizes our findings. 


\subsection{Observations}

\subsubsection{SDSS Photometry}

The SDSS (York et al., 2000; Stoughton et al., 2002; Pier et al., 2003; Ivezić et al., 2004 ) is a large ( 10,000 sq. deg.), multi-color (ugriz; Fukugita et al., 1996; Gunn et al., 1998; Hogg et al., 2001; Smith et al., 2002; Tucker et al., 2006) photometric and spectroscopic survey centered on the Northern Galactic Cap. The 2.5m telescope (Gunn et al., 2006), located at Apache Point Observatory scans the sky on great circles, as the camera (Gunn et al., 1998) simultaneously images the sky in five bands to a faint limit of $\sim 22.2$ in $r$, with a typical uncertainty of $\sim 2 \%$ at $r \sim 20$ (Ivezić et al., 2003; Adelman-McCarthy et al., 2006). The last data release (DR5 ${ }^{1}$; AdelmanMcCarthy et al., 2007a) comprises 8000 sq. deg. of imaging, yielding photometry of $\sim 217$ million unique objects, including $\sim 85$ million stars. SDSS photometry has enabled a myriad of studies on both Galactic structure (e.g. Yanny et al., 2000; Newberg et al., 2002; Juric et al., 2005; Belokurov et al., 2006) and low-mass stars (e.g. Hawley et al., 2002; Walkowicz et al., 2004; West et al., 2005; Davenport et al., 2006).

\subsubsection{SDSS Spectroscopy}

When sky conditions prohibit photometric observations, the SDSS telescope is fitted with twin fiber-fed spectrographs. These instruments simultaneously obtain 640 medium-resolution $(R \sim 1,800)$, flux-calibrated, optical (3800-9200 $\AA$ ) spectra per $3^{\circ}$ plate, permitting radial velocity measurements for most stars with an uncertainty of $\sim 10 \mathrm{~km} \mathrm{~s}^{-1}$ (Abazajian et al., 2004). A typical 45 minute observation yields a signal-to-noise ratio per pixel $>4$ at $g=20.2$ and $i=19.9$ (Stoughton et al., 2002), with a broadband flux calibration uncertainty of $\sim 4 \%$ (Abazajian et al., 2004). The

\footnotetext{
${ }^{1}$ http://www.sdss.org/dr5/
} 
DR5 sample includes over 1 million spectra, with $\sim 216,000$ stellar spectra (AdelmanMcCarthy et al., 2007a). The majority of spectra in the SDSS database are drawn from 3 main samples, which target objects based on their photometric colors and morphological properties. These samples are optimized to observe galaxies (Strauss et al., 2002), luminous red galaxies with $\mathrm{z} \sim 0.5-1.0$ (Eisenstein et al., 2001), and high redshift quasars (Richards et al., 2002). However, low-mass stars have similar colors to some of these samples and therefore are observed serendipitously. SDSS spectroscopy of late-type dwarfs has been the focus of numerous studies (see Hawley et al., 2002; Raymond et al., 2003; West et al., 2004; Silvestri et al., 2006; West et al., 2006; Bochanski et al., 2007b and references therein).

\subsubsection{SDSS Low-Mass Spectroscopic Sample: SLoMaSS}

During Fall 2001, a call was placed to the SDSS collaboration to design special spectroscopic plates that employed different targeting algorithms than the usual SDSS survey samples described in $§ 3.3 .2$. We designed and proposed a series of observations to probe the local vertical structure of the Milky Way, obtaining spectra of low-mass dwarfs in the southern equatorial stripe (stripe 82) of the SDSS photometric footprint during Fall 2002. This stripe at zero declination is repeatedly observed during the time of the year when the Northern Galactic Cap is not visible. These repeat scans sample an area of $~ 300$ sq. deg. and have been used to study Type Ia supernovae (Sako et al., 2005; Frieman et al., 2007), stellar variability (Sesar et al., 2007) and characterize the repeatability of SDSS photometry (Ivezić et al., 2007). SLoMaSS is comprised of stars with unsaturated griz photometry and extinction-corrected magnitude limits of $15<i<18$ and $i-z>0.2$. A series of three spectroscopic tilings composed of 15 plates (numbers 1118-1132, centered on $l \sim 105^{\circ}, b \sim-62^{\circ}$ ) was observed, yielding a total of 8880 stellar spectroscopic targets in SLoMaSS, each with ugriz photometry. 


\subsection{Analysis}

\subsubsection{Spectral Types}

The spectroscopic data were analyzed with the HAMMER suite of software (Covey et al., 2007a) which measures spectral type, $\mathrm{H} \alpha$ emission line properties, and various spectral band indices. This pipeline automatically assigns a spectral type to each star, then allows the user to confirm and adjust the spectral type manually, if necessary. The accuracy of the final spectral types is \pm 1 subtype. After examining the entire sample by eye, we selected spectroscopically confirmed $\mathrm{K}$ and $\mathrm{M}$ dwarfs. This cut decreased the sample from 8880 to 8696 stars. The final spectral type distribution of SLoMaSS, after the additional cuts explained below, together with the average $i-z$ color for each spectral type bin, is shown in Figure 3.1.

\subsubsection{Distances - Photometric Parallax}

Distances to the SLoMaSS stars were determined using the photometric parallax relations described in West et al. (2005) and Davenport et al. (2006). The West et al. (2005) relation was employed for stars with $i-z$ redder than 0.37 and the Davenport et al. (2006) parallax relation was applied to the bluer stars in SLoMaSS. We neglect reddening corrections, since the average extinction computed for the total column along this line of sight from Schlegel et al. (1998) was small $(<0.05$ in $i)$, and most of these stars will lie in front of significant amounts of dust. Stars with $i-z$ colors that did not fall within the appropriate boundaries of the photometric parallax relations $(0.22<i-z<1.84)$ were removed from the sample, decreasing its size to 8280 stars. Additionally, we applied the photometric white dwarf-M dwarf pair cuts of Smolčić et al. (2004), removing 4 more stars from the sample. The $i, i-z$ Hess diagram and $r-i, i-z$ color-color diagram are shown in the lower panels of Figure 3.1. The

distance from the Galactic Plane was computed assuming the Sun's vertical position to be 15 pc above the Plane (Cohen, 1995; Ng et al., 1997; Binney et al., 1997). The 
vertical distribution of stars in SLoMaSS is shown in Figure 3.2. Note that the decline in stellar density at large distances reflects the incompleteness of our sample, not the intrinsic stellar density, while the decline at small distances from the Plane is due to the saturation of SDSS photometry.

\subsubsection{Distances - Spectroscopic Parallax}

In addition to the photometric parallax relations mentioned above, we also computed the distances to the M dwarfs in SLoMaSS using the spectroscopic parallax relations of Hawley et al. (2002). These distances served as a check on the photometric parallax relations and were later used to divide the sample in a search for color differences between the thin and thick disks (see $§ 3.5 .3$ for details). The spectroscopic parallax of $\mathrm{K}$ stars was not computed, as no reliable calibrated relations were available.

\subsubsection{Radial Velocities, Proper Motions \& UVW Velocities}

Radial velocities were computed by cross-correlating the $\mathrm{M}$ dwarf stellar spectra against the low-mass template spectra of Bochanski et al. (2007b) with the IRAF ${ }^{2}$ task fxcor. This cross-correlation routine is based on the method described in Tonry $\&$ Davis (1979). The measured velocities have external errors of $\sim 4 \mathrm{~km} \mathrm{~s}^{-1}$. The K dwarf radial velocities were obtained directly from the SDSS/Princeton 1D spectral pipeline $^{3}$, with typical uncertainties of $10 \mathrm{~km} \mathrm{~s}^{-1}$.

Proper motions were determined from the SDSS+USNO-B proper motion catalog (Munn et al., 2004). The catalog is $~ 90 \%$ complete over the magnitude limits of our sample, with random errors of $\sim 3.5$ mas $\mathrm{yr}^{-1}$. We removed an additional 878 stars with poorly measured proper motions ${ }^{4}$, reducing our final sample size to 7398 stars.

\footnotetext{
${ }^{2}$ IRAF is distributed by the National Optical Astronomy Observatories, which are operated by the Association of Universities for Research in Astronomy, Inc., under cooperative agreement with the National Science Foundation.

${ }^{3}$ http://spectro.princeton.edu

${ }^{4}$ Specifically, we required a match between catalogs (match $>0$ ), detections in at least 4 plates
} 
Combining distances, proper motions and radial velocities, we computed the $U V W$ space motions of each star in SLoMaSS using the method of Johnson \& Soderblom (1987). The velocities are computed in a right-handed coordinate system, with positive $U$ velocity directed toward the Galactic center and corrected for the solar motion $\left(10,5,7 \mathrm{~km} \mathrm{~s}^{-1}\right.$; Dehnen \& Binney, 1998) with respect to the local standard of rest.
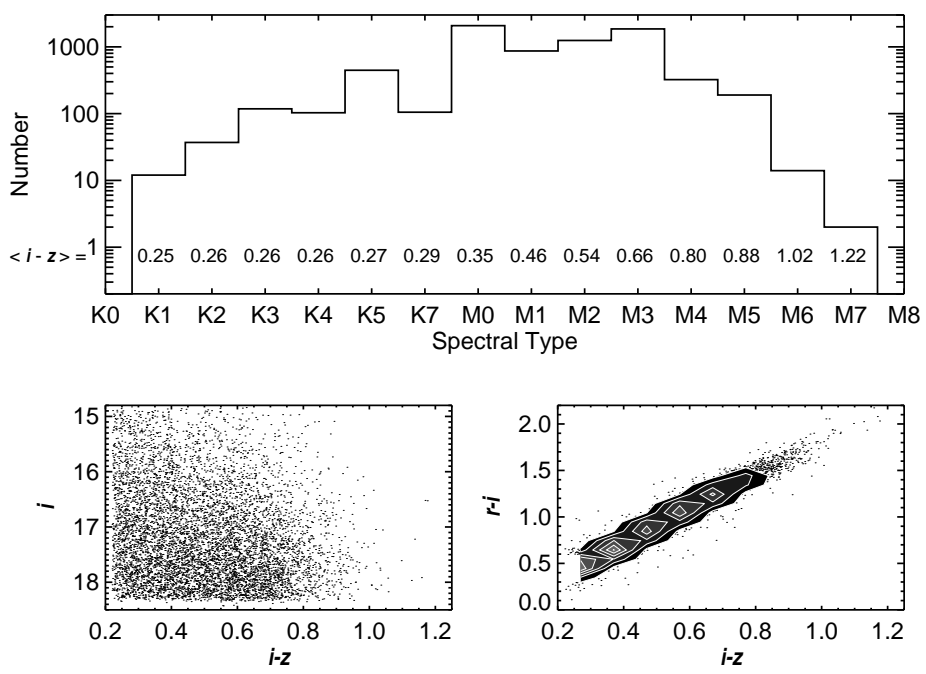

Figure 3.1 Upper panel: Distribution of spectral types within SLoMaSS. The mean $i-z$ color at each spectral type is shown above each bin. Lower panels: $i$ vs. $i-z$ colormagnitude distribution and $r-i$ vs. $i-z$ color-color diagram of stars in SLoMaSS. The lowest contour (black) in the color-color diagram indicates a density of 100 stars in a square 0.1 magnitudes wide. Each additional contour represents 100 additional stars.

\subsection{Results}

The SLoMaSS observations were used to investigate kinematics, colors, chromospheric activity and metallicity in the thin and thick disk populations.

in USNO-B (nfit $\geq 5$ ), no other objects within 7 arcseconds, which is the resolution of the POSS plates (dist22 > 7; see Kilic et al., 2006), and small errors in the proper motion determination ( $\operatorname{sigRA}<1000$ and sigDec $<1000$ ); see (Munn et al., 2004) for details. 


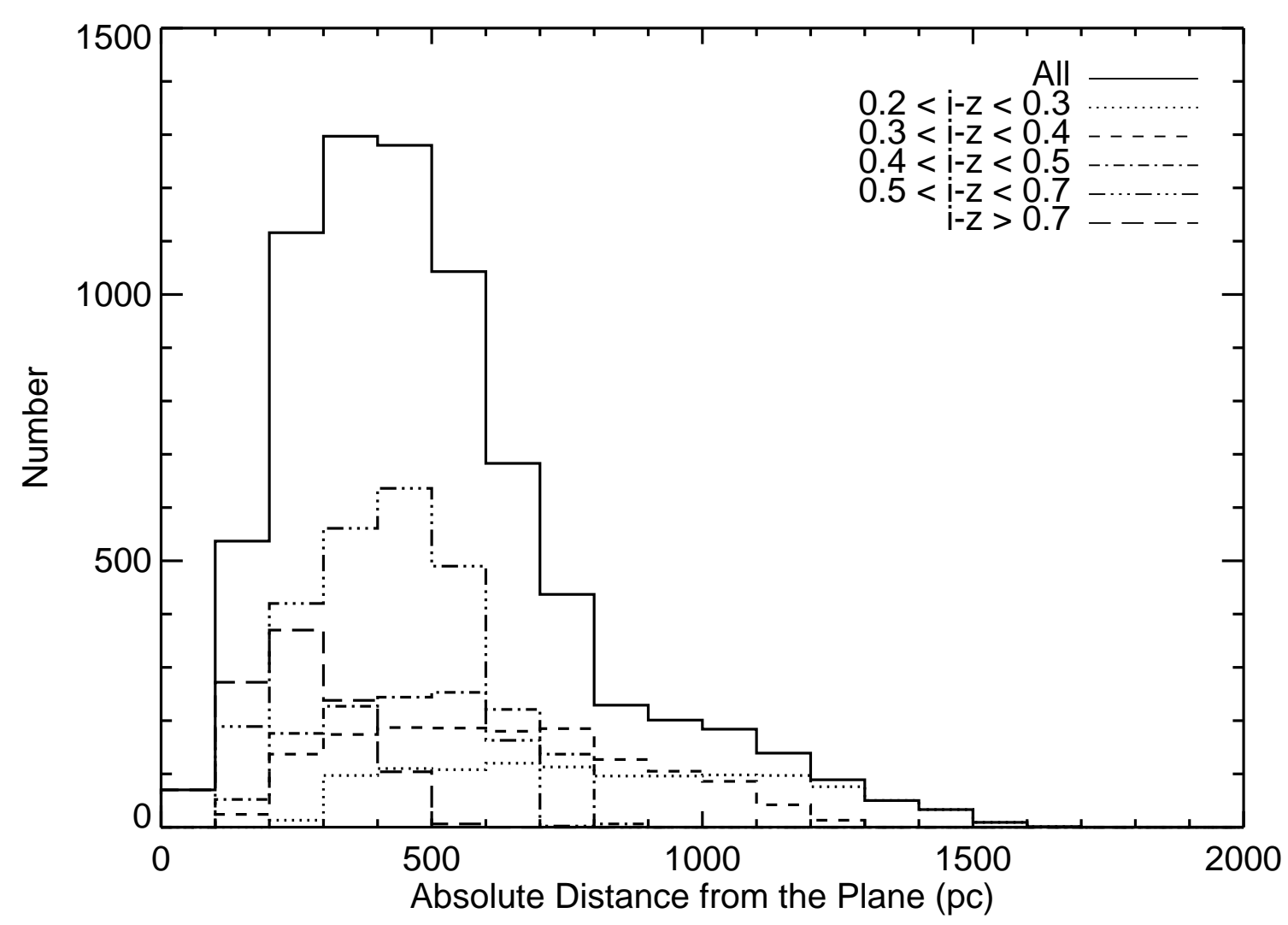

Figure 3.2 The absolute vertical distance distribution of the sample binned every $100 \mathrm{pc}$. The solid thick histogram is the total distribution, while the five remaining histograms represent the height distribution for five $i-z$ color bins as described in the legend. Note that there are hundreds of stars per bin out to an absolute vertical distance of $\sim 1000 \mathrm{pc}$. 
We used two methods in our analysis. First, we examined the velocity distributions with no assumptions regarding the parent population of a given star (see §3.5.1). These kinematic results were then used to test a leading Galactic model (§3.5.2). We also used the space motion of each star to assign it to the thin or thick disk population, and investigated the color, metallicity and activity differences between the two populations $(\S 3.5 .3)$.

\subsubsection{Kinematics}

The sample was binned in 100 pc increments of vertical distance from the Galactic Plane and probability plots of $U V W$ velocities were constructed (Lutz \& Upgren, 1980; Reid et al., 1995a, 2002). These diagrams plot the cumulative probability distribution in units of the standard deviation of the distribution. Hence, a single Gaussian distribution will appear as a straight line with a slope correpsonding to the standard deviation of the sample and the y-intercept equal to the median of the distribution. Non-Gaussian distributions will significantly deviate from a straight line. An example of velocity distributions and their probability plots are shown in Figure 3.3. An advantage to this method is its immunity to outliers and to binning effects from poorly populated histograms.

For each distance bin in SLoMaSS, the probability plots are well fit with two lines: a low-dispersion, kinematically colder "core" component $(<|1 \sigma|)$ and a highdispersion, "wing" component $(>|1 \sigma|)$. Shown in Figure 3.4 is an example of our analysis, illustrating linear fits to the core and wing distributions. At larger Galactic heights, the wing component traces the in situ thick disk population.

The resulting dispersions are shown as a function of distance from the Galactic Plane in Figure 3.5. The low-dispersion core component is well-behaved, smoothly increasing with increasing height. The high-dispersion wing component is subject to larger scatter, but generally increases with height from the Galactic Plane. Our results are summarized in Table 3.1. In order to facilitate comparison to the previous 
results included in the Table, we report the dispersions for several distance bins, as well as the entire sample.

\subsubsection{Galactic Dynamical Models}

\section{Besançon Model: Introduction}

Our sample is well suited to rigorously test current Galactic models. We chose the Besançon model (Robin et al., 2003) as a fiducial, since it simulates both star counts and kinematics. This model is constructed on several assumptions and empirical constraints, with the goal of reproducing the stellar content of the Milky Way. Four populations comprise the model: the thin and thick disks, bulge and halo. For each population, a star-formation history, initial mass function, density law and age are imposed. Additionally, an age-metallicity relation is employed for each population, with a Gaussian dispersion about the mean metallicity of each component (see Tables 1 and 3 of Robin et al., 2003).

Kinematically, the thin disk is composed of 7 groups of different ages (from 0.0 to 10 Gyr), each being isothermal except for the youngest (0.0 - 0.15 Gyr). The thick disk is composed of an 11 Gyr old population, with a velocity ellipsoid based on the measurements of Ojha et al. $(1996,1999)$. An age-velocity dispersion relation from Gomez et al. (1997) is imposed on each component, and the model is then allowed to self-consistently evolve to the present-day. This self-consistency is achieved with the method described in Bienayme et al. (1987). Stellar populations are formed according to their appropriate density laws and introduced with an initial velocity dispersion. The mass density of the stars is summed in a column of unit volume centered on the Sun along with dark matter halo and interstellar material contributions, and the potential is computed using the Poisson equation. Stars are then evolved using the

collisionless Boltzmann equation in this new potential, and redistributed in the $z$ direction, as complete orbital evolution is not included in this model. The process 

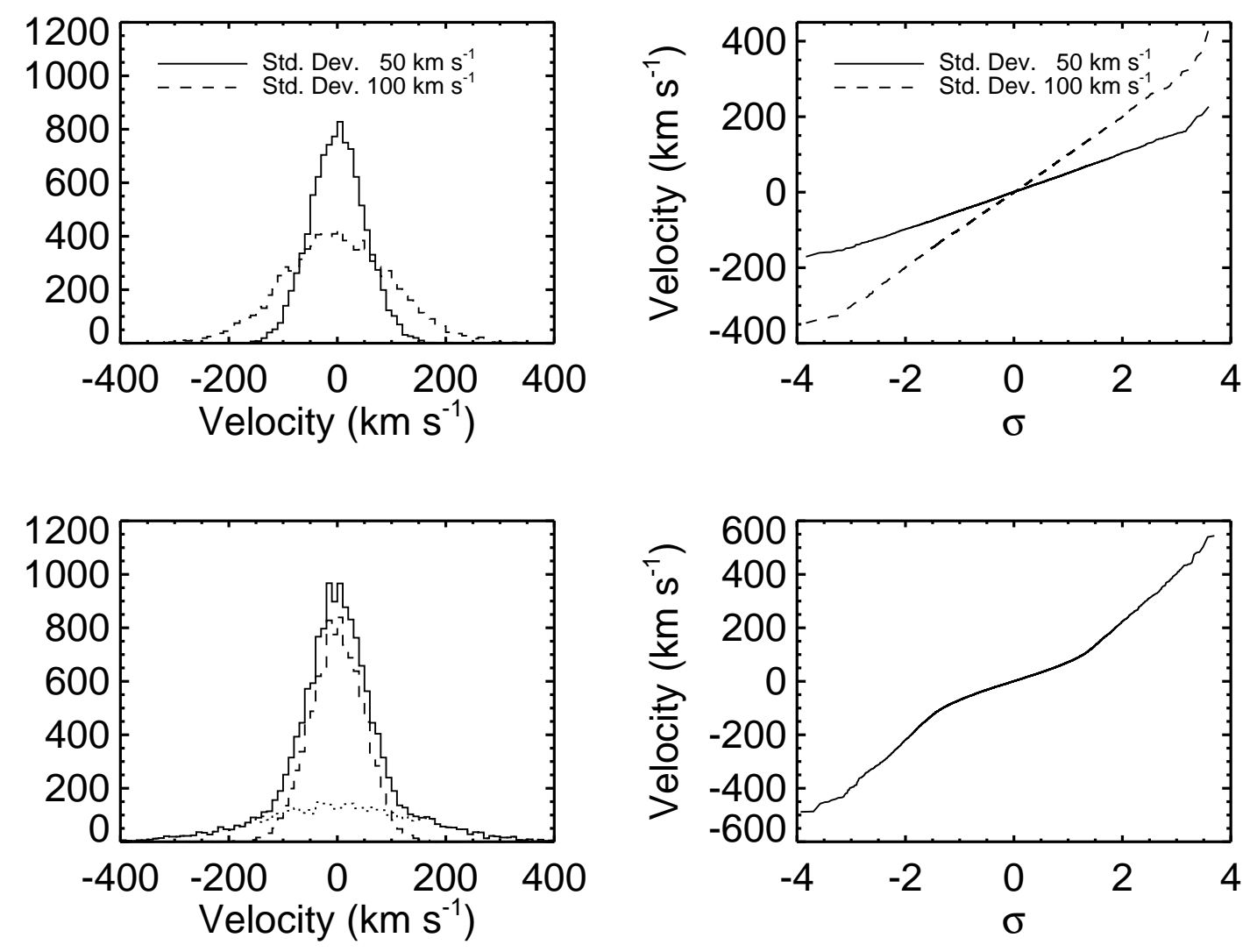

Figure 3.3 Schematics of velocity distributions (left panels) and their corresponding probability plots (right panels). The upper row displays two Gaussian distributions, one with a standard deviation of $\sim 50 \mathrm{~km} \mathrm{~s}^{-1}$ (solid line) and another with a standard deviation of $\sim 100 \mathrm{~km} \mathrm{~s}^{-1}$ (dashed line). A straight line in the probability plot signifies an underlying Gaussian distribution and the slope of the line is a measure of the standard deviation of the distribution. Note that the slope of the dashed line is twice that of the solid line. In the bottom row, a low dispersion component (dashed line) and high dispersion component (dotted line) are summed to produce the solid histogram. While the dual nature of the distribution is not readily apparent in the solid histogram, it is easily detected in the probability plot. 

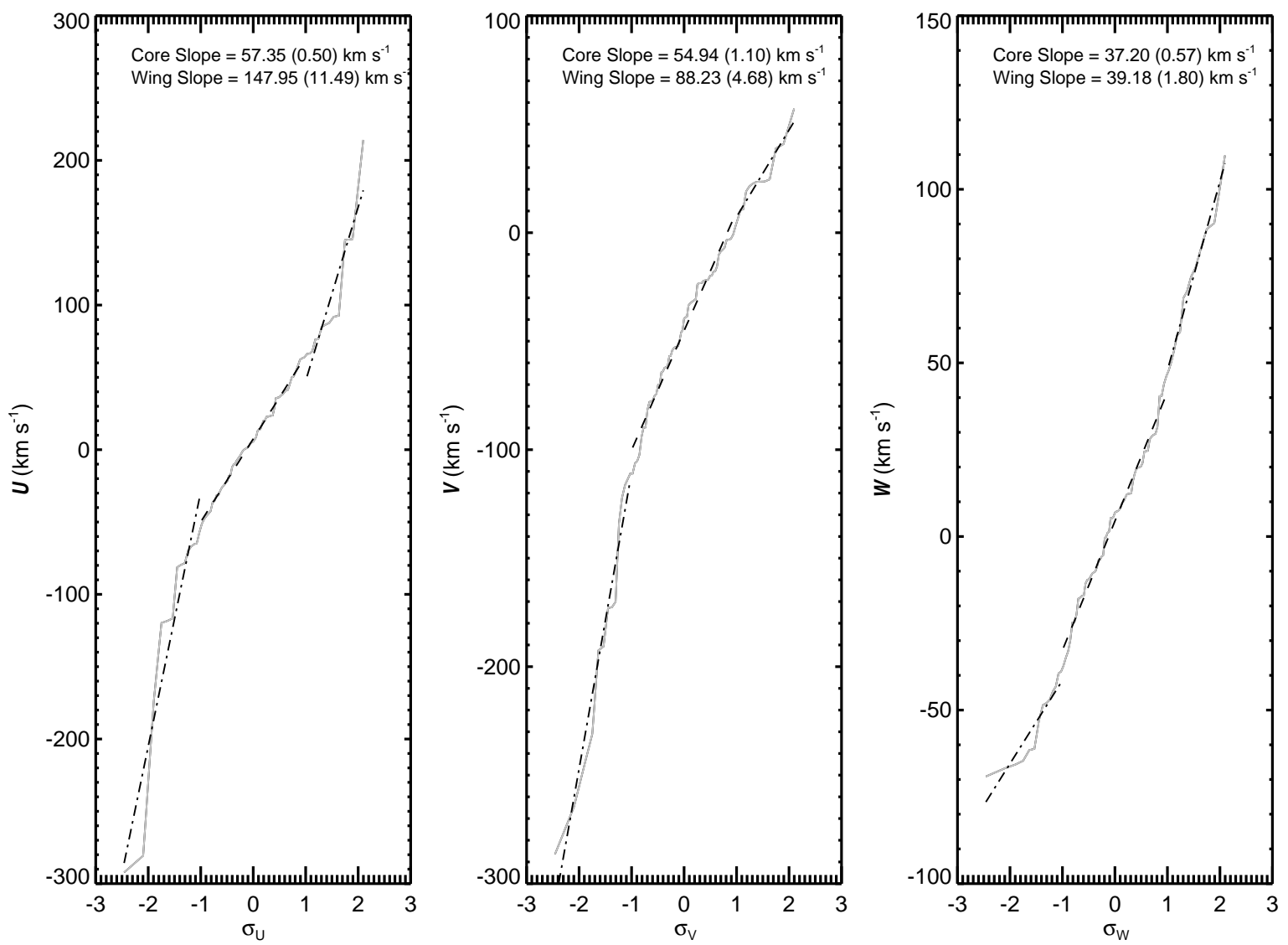

Figure 3.4 Illustrative example of our velocity dispersion analysis. Shown are probability plots for $U, V$ and $W$ for the $1200<z<1300 \mathrm{pc}$ bin (solid lines). The "core" component (dashed line) is fit between $\pm 1 \sigma$, while the "wing" component (dotdashed line) fits the outer edges of the distribution. Note the strong asymmetric drift component manifested as a change in slope at negative $V$ velocities. 

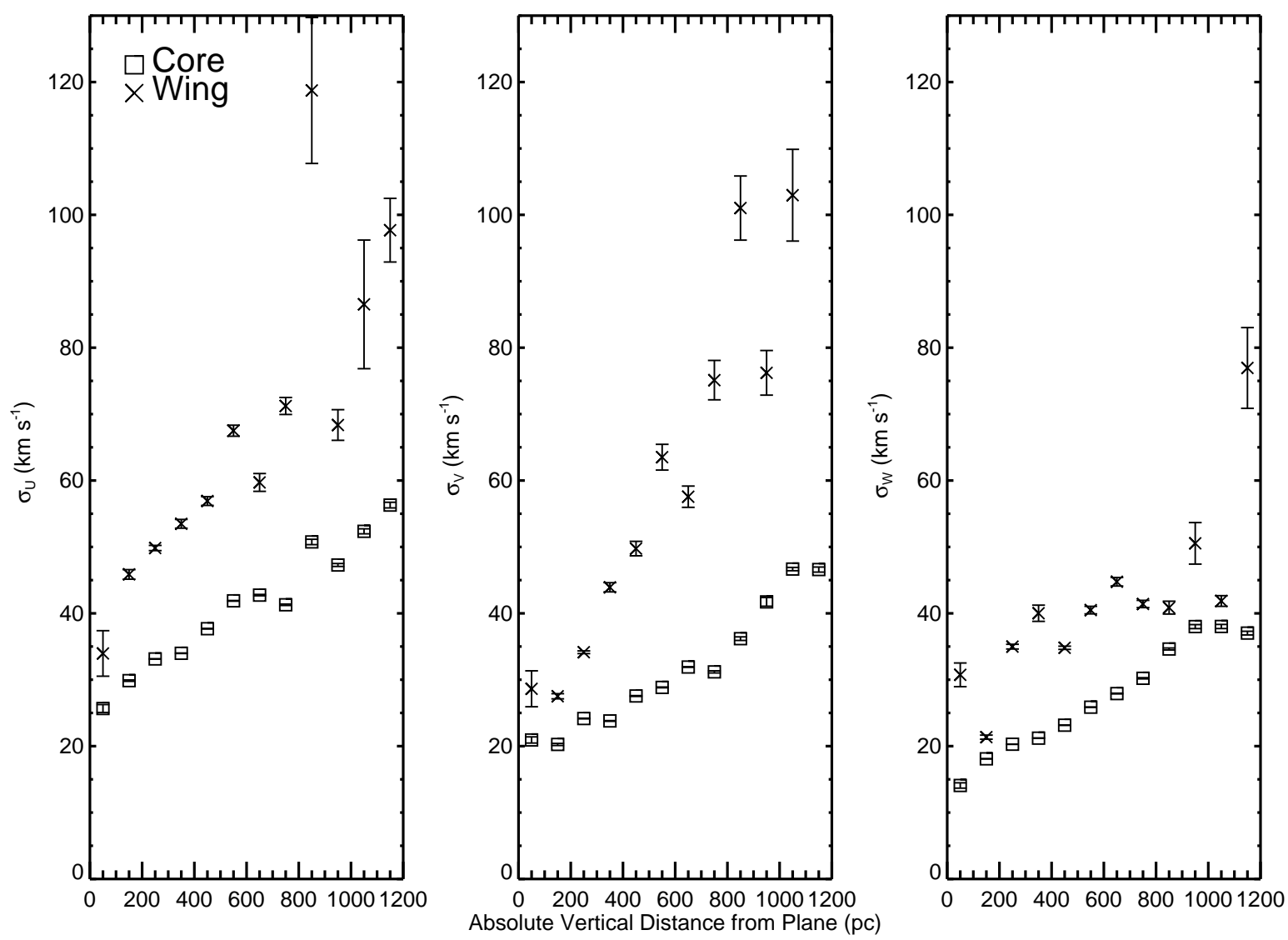

Figure 3.5 Velocity Dispersion as a function of vertical distance from the plane in 100 pc bins. The open squares represent the kinematically colder, core component and the crosses represent the high-dispersion, wing component. Errors are derived from the linear fits to the probability plots. Note the smooth trend in the low-dispersion component as a function of height from the Plane. 
is iterated until the potential and scale heights of the disk populations converge at the $1 \%$ level. The local-mass density, which was empirically determined by Creze et al. (1998), also imposes a constraint on the initial Galactic potential. We note that imposing an age-velocity dispersion relation on the model dominates the kinematics, and that there are significant uncertainties in these relations. For example, the agevelocity dispersions used by Rocha-Pinto et al. (2004) differ by factors of $\sim 2$ for the oldest stars, compared to the relations in Gomez et al. (1997). These discrepancies are primarily derived from the difficulty in determining ages of field stars, with systematic differences imposed by various methods (i.e. isochrone fitting vs. chromospheric ages).

Intrinsic properties such as age, mass, luminosity, metallicity, position and velocity are available for each star in the simulation. Observable properties, such as colors, proper motions and radial velocities are also reported. To determine colors, the model uses the Lejeune et al. (1998) database, which employs adjusted stellar synthetic spectra that attempt to match empirical color-temperature relations.

\section{Comparison to SDSS}

We queried the Besançon webpage ${ }^{5}$, generating a suite of synthetic datasets along the appropriate Galactic sightline of SLoMaSS $\left(l \sim 105^{\circ}, b \sim-62^{\circ}\right)$ with proper magnitude limits and error characteristics. We included both the thin and thick disks in the model inputs. The bulge and halo components were excluded since SLoMaSS points away from the bulge, and with a maximum distance of $\sim 2000 \mathrm{pc}$, we expect halo contamination to be minimal. A total of 25 models were generated from identical input, in order to minimize Poisson noise.

As a consistency test of the Besançon model, we compared the model star counts to those obtained with SDSS survey photometry in the SLoMaSS field. We queried the

\footnotetext{
${ }^{5}$ http://physique.obs-besancon.fr/modele/
} 
SDSS Catalog Archive Server ${ }^{6}$ for good point spread photometry ${ }^{7}$ of stars along the appropriate sightline. This query resulted in 46,730 stellar targets. In the models, the mean star count was 46,991 with a standard deviation of 137 . This excellent agreement should be viewed cautiously, as only rough maginitude cuts were imposed on the Besançon models, and we did not attempt to model observational problems that would affect SDSS star counts (Vanden Berk et al., 2005), such as cosmic rays or diffraction spikes near bright stars. However, the test inspires confidence that the Besançon model adequately represents the observed SDSS star counts.

\section{Comparison to SLoMaSS}

Each of the 25 models was sub-sampled to reproduce the color and distance distributions observed in SLoMaSS. The two-dimensional color-distance density distribution was computed for SLoMaSS, and color-distance pairs were randomly drawn according to this normalized density distribution. If the color-distance pair was found in the Besançon model, then it was kept. This Monte-Carlo sampling continued until there were 7398 color-distance pairs, matching the number of stars in SLoMaSS. This sampling forced each model to simulate the properties of the SLoMaSS observations when compared to the complete SDSS photometry . Since SDSS $r-i$ colors are not available for the Besançon model, the SLoMaSS colors were transformed to $R-I$ using the relations of Davenport et al. (2006). Shown in Figure 3.6 are the $R-I$ and distance distributions for one instance of the model, along with those from SLoMaSS. Thus, each model is "observed" in a manner consistent with the spectroscopic observations of the SDSS field photometry.

\footnotetext{
${ }^{6}$ http://cas.sdss.org/dr5/en/

${ }^{7}$ Specifically, we required the following flags: a detection in BINNED1 and no EDGE, NOPROFILE, PEAKCENTER, NOTCHECKED, PSF_FLUX_INTERP, SATURATED, BAD_COUNTS_ERROR, DEBLEND_NOPEAK, CHILD, BLENDED, INTERP_CENTER or COSMIC_RAY flags set (Stoughton et al., 2002).
} 


\section{Kinematic Comparisons}

Using the sub-sampled model data, we compared the kinematic predictions of the Besançon models to the $U V W$ velocities of SLoMaSS. Using the method described in §3.5.1, we constructed two sets of probability plots for each model: one composed of the thin and thick disk stars and one measured solely for the thin disk. The reason for this separation was twofold. The first was to isolate any systematics between the models thin and thick disk predictions. Additionally, this allowed for direct testing comparison of the thin disk predictions, since most of these stars lie on the inner "core" region of the probability plot. That is, if the velocity predictions are correct for the thin disk, the overall slope of the isolated thin disk models should roughly match the "core" slope measured from SLoMaSS.

In Figure 3.7, an illustrative example of this analysis is shown. The main effect of adding the thick disk component is to increase the slope of both the core and wing components. Additionally, the wing component is enhanced, as seen in the $W$ velocity distribution, which is expected from addition of a high dispersion population. It is clear from comparing to Figure 3.4 that the combination of the thin and thick disk model predictions are necessary to simulate the structure seen in the SLoMaSS data.

Following the method explained in $§ 3.5 .1$ we measured the slopes of the "core" and "wing" components of the each model as a function of distance from the Galactic Plane. The results of this analysis, compared to the SLoMaSS results are shown in Figures 3.8 and 3.9. The SLoMaSS results (open squares), which are shown in Figure 3.5, are compared to the average Besançon prediction (crosses) for the thin (upper panels) and thick disk (lower panels). While the model does well in predicting general trends, there are clearly some systematics. The model predictions for the $\sigma_{W}$ thin disk velocity dispersions are systematically low, suggesting there may be flaws in the method used to compute these motions. Additionally, the model underestimates the thick disk $\sigma_{V}$ dispersions at large Galactic heights. As described above (§3.5.2), the 
assumed age-velocity dispersions relations, which dominate the predicted kinematic structure, are uncertain and may contribute to this disagreement. This speculation is supported by the results summarized in Table 3.1, which demonstrate that most previous surveys (as well as our own) have measured velocity dispersions significantly higher than those predicted from the model. The mean velocity distributions for both SLoMaSS and the Besançon model are shown in Figure 3.9. Again, the model in general performs well, but there are evident systematic differences. The mean $V$ velocites predicted for the thin disk by the model are systematically high, and the thick disk $V$ velocities diverge at large Galactic heights. The first difference may be attributed to our chosen solar motion values $\left(10,5,7 \mathrm{~km} \mathrm{~s}^{-1}\right.$; Dehnen \& Binney, 1998). A larger adopted value of $V_{\odot}$, such as the classic value of $12 \mathrm{~km} \mathrm{~s}^{-1}$ (Delhaye, 1965), would move the mean velocities towards agreement. The discrepancy in the thick disk $V$ velocities is probably due to small number statistics, as seen in Figure 3.2.

\subsubsection{Differences between the Thin and Thick Disks}

In order to examine observable differences between $M$ dwarf members of the thin and thick disks, we kinematically separated the sample using the method of Bensby et al. (2003). This technique selects outliers in the wings of the three-dimensional Gaussian velocity distribution, and computes the probability of these stars belonging to the thick disk. The thin and thick disk populations were characterized by the velocity dispersions in Bensby et al. (2003). In order to minimize systematics, the $U V W$ velocities were re-computed using distances determined with the spectroscopic parallax relations of Hawley et al. (2002), as described above in §3.4.3. Thus, $U V W$ should not a priori vary systematically with color. This also limits the analysis to the $6577 \mathrm{M}$ dwarfs in the sample. 

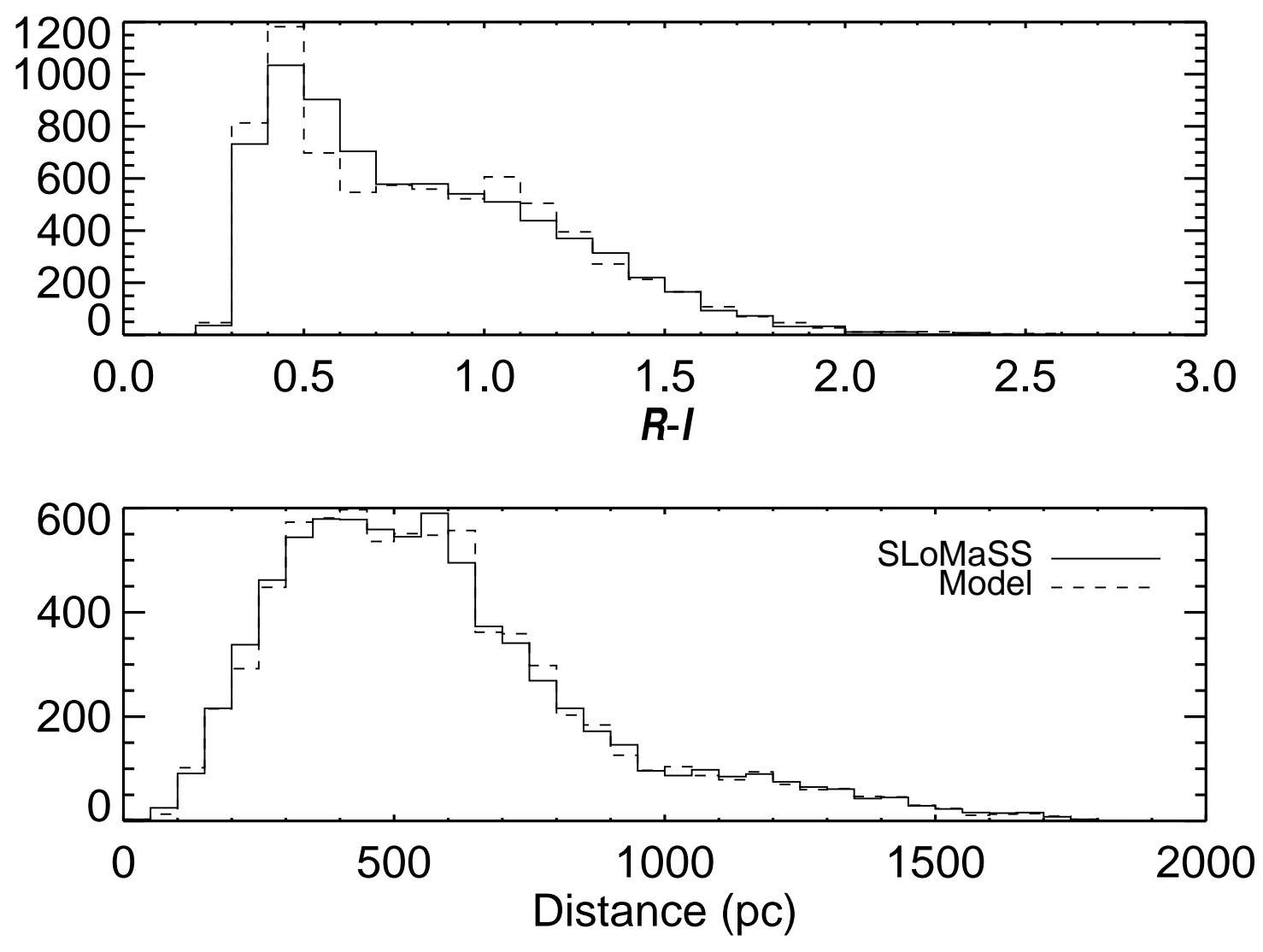

Figure 3.6 Shown are the $R-I$ and distance distributions of the stars in SLoMaSS (solid line) and a sampled model (dashed line). Note the agreement between the two datasets, indicating that we are sampling each instance of the Besançon model in a manner consistent with the spectroscopic observations in SLoMaSS. 

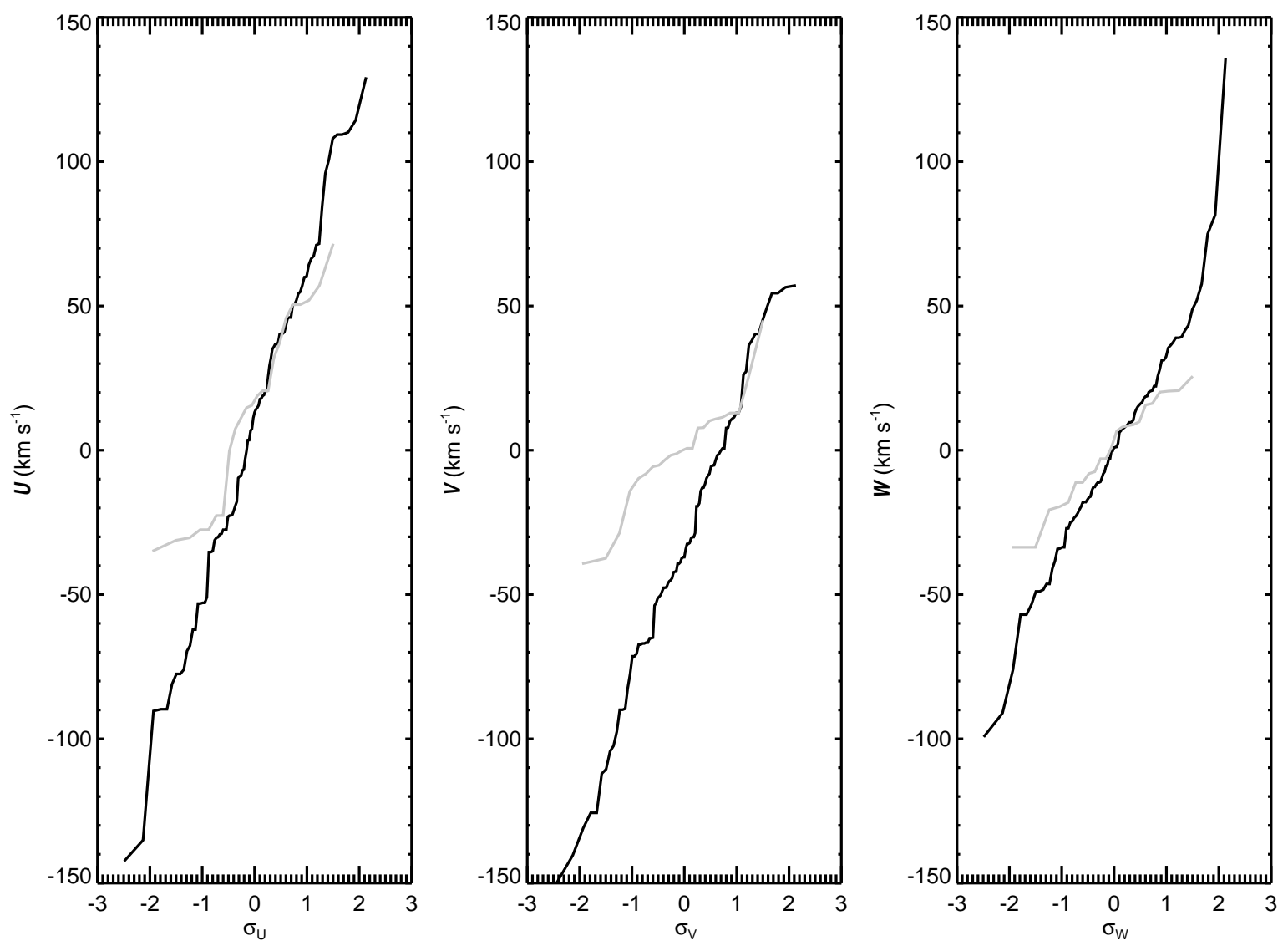

Figure 3.7 Probability Plots of the $U, V$ and $W$ velocites for pure thin disk (shaded line) and thin + thick models (solid line) in the $1200<z<1300$ pc bin. This height bin corresponds to the SLoMaSS data shown in Figure 3.4. Note that when the thick disk is added to the distribution, the overall slope increases, and the wing components are enhanced, as seen in the $W$ probability plot. The combined thin and thick disk velocity distributions are also necessary to match the two-component structure seen in the SLoMaSS probability plots. 

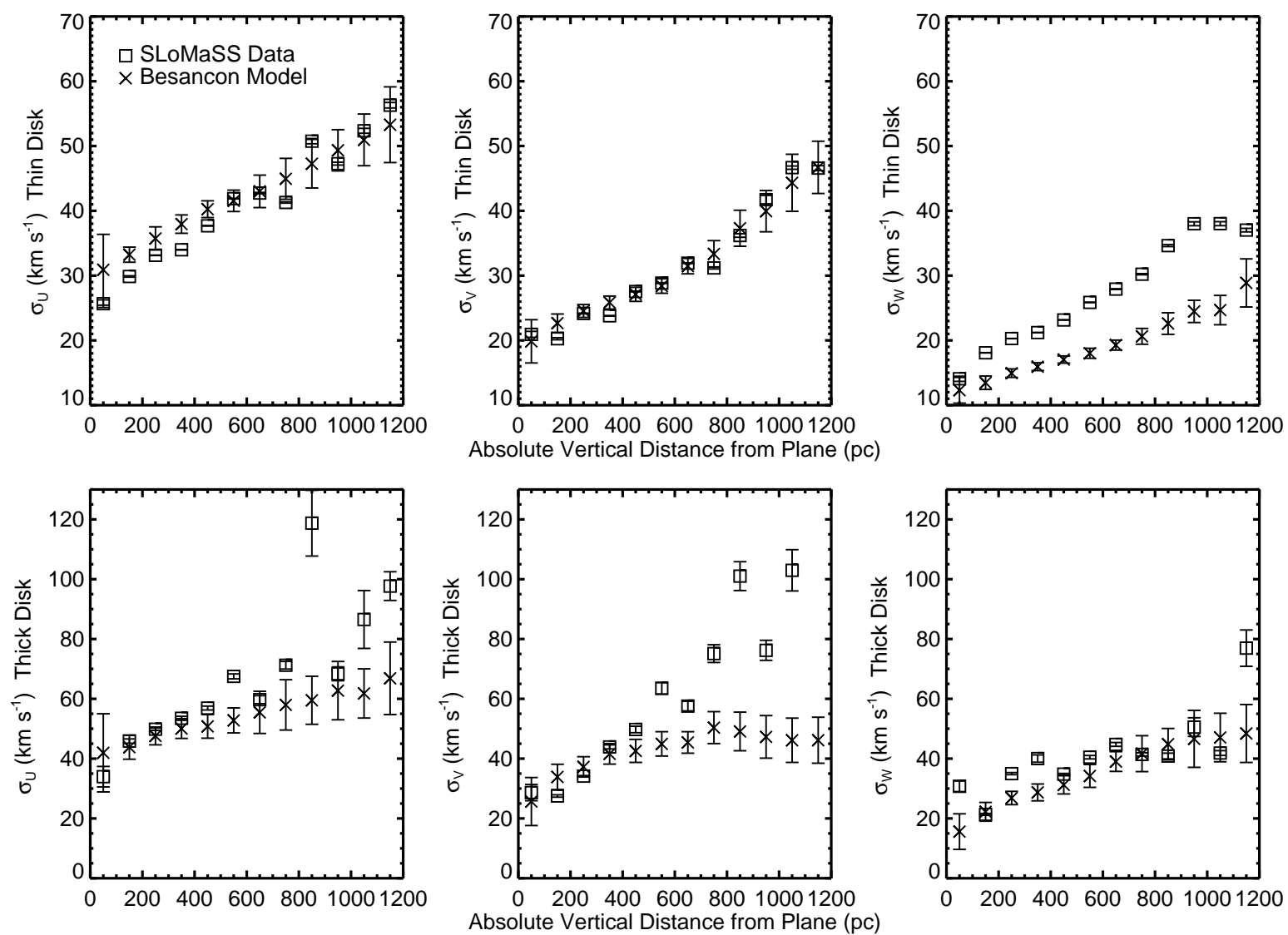

Figure 3.8 Thin Disk (upper panels) and Thick Disk (lower panels) velocity dispersions for SLoMaSS data (open squares) and Besançon model predictions (crosses). The model reproduces general trends, such as increased dispersion with distance from the Galactic Plane. The thin disk $W$ velocity dispersions are systematically low, as are the thick disk $V$ dispersions at large heights. This is probably due to the assumed age-velocity dispersion relation, as explained in $§ 3.5 .2$. 

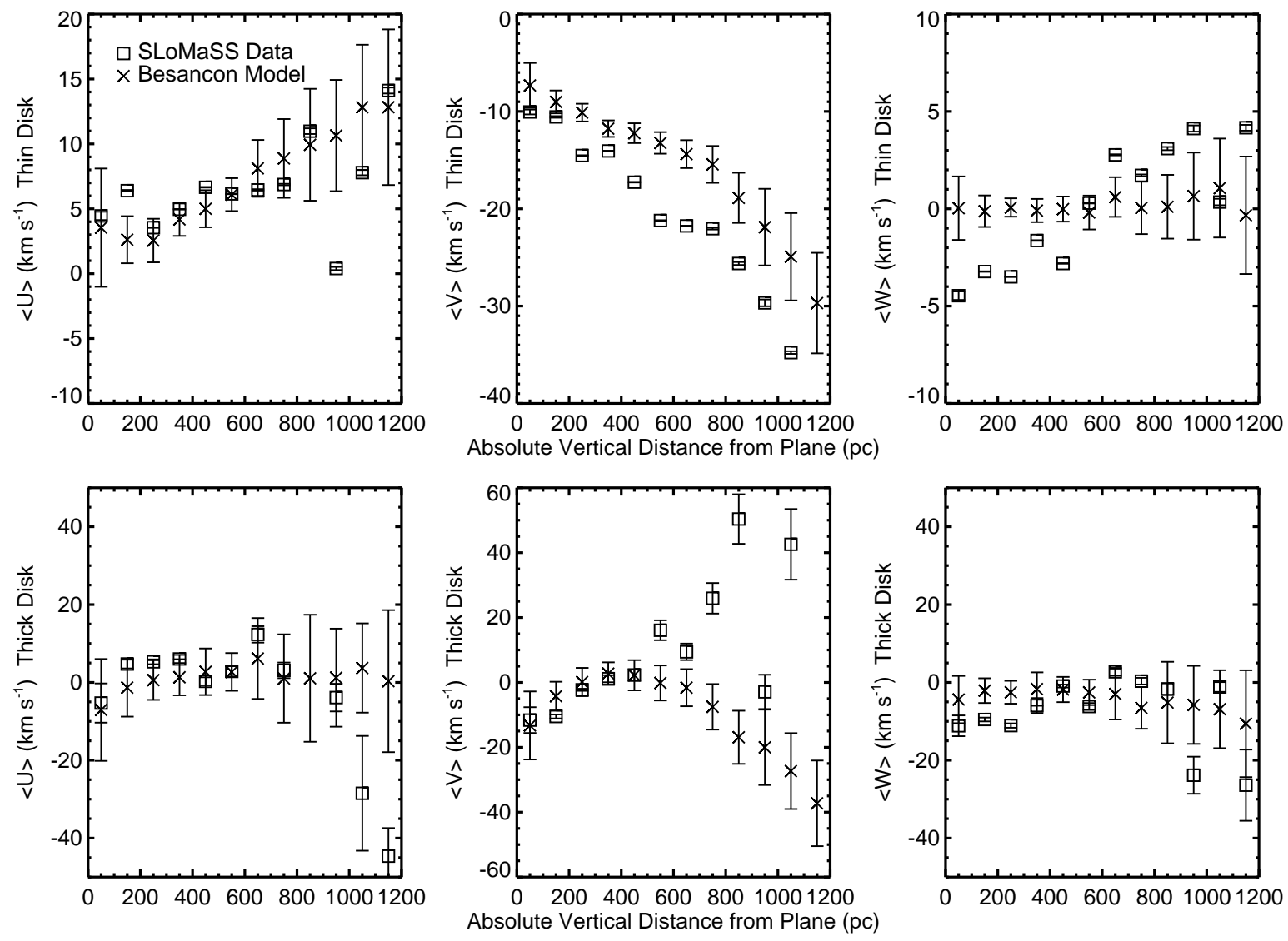

Figure 3.9 Thin Disk (upper panels) and Thick Disk (lower panels) mean velocities for SLoMaSS data (open squares) and Besançon model predictions (crosses). The thin disk mean $V$ velocities predicted by the Besançon models are systematically low, while the observed thick disk mean $V$ velocities diverge at large Galactic heights. 


\section{Metallicity}

The exact formation mechanism of the thick disk is uncertain (see Majewski, 1993 and references therein), but there is evidence that the mean metallicity of the thick disk is lower than that of the thin disk (Reid \& Majewski, 1993; Chiba \& Beers, 2000; Bensby et al., 2003). Additionally, differences in the $\alpha$-element distributions have been shown to be distinct (Fuhrmann, 1998; Feltzing et al., 2003; Bensby et al., 2003). While direct metallicity determinations of $\mathrm{M}$ dwarfs are difficult (Woolf \& Wallerstein, 2006), proxies of metallicity have been employed (Gizis, 1997; Lépine et al., 2003a; Burgasser \& Kirkpatrick, 2006). These previous studies have used the low-resolution molecular band indices (CaH1, CaH2, CaH3, and TiO5) defined in Reid et al. (1995a) to roughly discriminate between solar-metallicity, subdwarf $([\mathrm{m} / H] \sim$ $-1.2)$ and extreme-subdwarf $([m / H] \sim-2)$ populations. Adapting the methods of Lépine et al. (2003a) and Burgasser \& Kirkpatrick (2006) we computed the ratio $(\mathrm{CaH} 2+\mathrm{CaH} 3) / \mathrm{TiO} 5$ for each star in the sample, which varies such that a larger value indicates a higher metallicity. The mean ratio for each spectral type for the thin and thick disk populations is shown in Figure 3.10. The two populations do not separate within the errors, suggesting that the observed metallicity distributions do not differ greatly.

\section{Color Gradients}

A lower mean metallicity could also manifest itself as a color shift at a given spectral type. Specifically, West et al. (2004) showed that at a given $r-i$ or $i-z$ color, low metallicity subdwarfs $([m / H] \sim-1.2)$ were redder in $g-r$. After SLoMaSS was kinematically separated, the mean $u-g, g-r, r-i$ and $i-z$ colors of the thin and thick disk stars were computed at each spectral subtype, as shown in Figure 3.11. There are no significant color differences between the thin and thick disks at a given spectral type. This further suggests that the SLoMaSS stars are not probing a large 


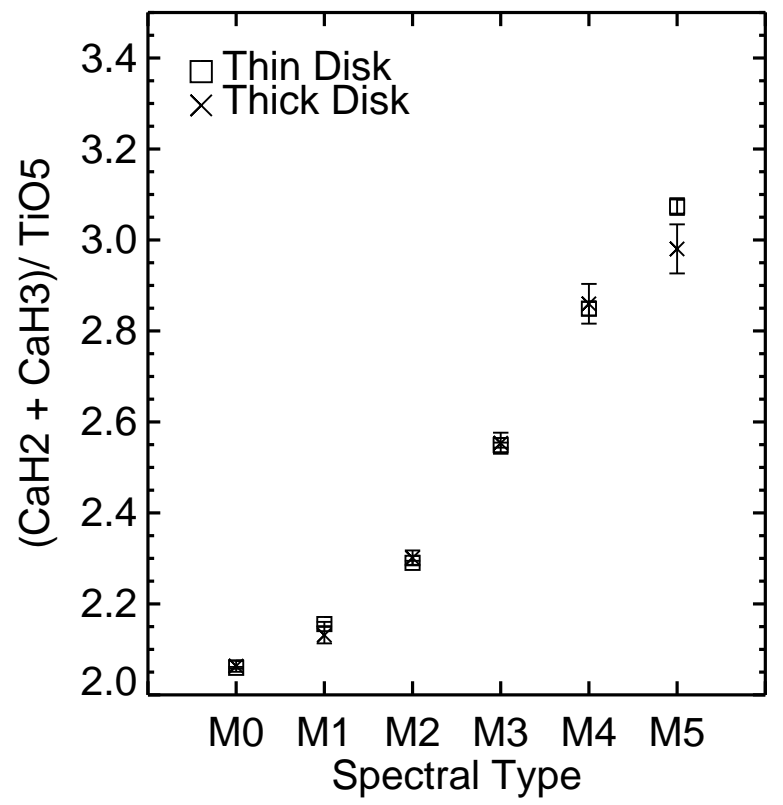

Figure 3.10 The metallicity-sensitive ratio of $(\mathrm{CaH} 2+\mathrm{CaH} 3) / \mathrm{TiO} 5$ vs. Spectral Type for the thin (open squares) and thick (crosses) disk populations. Higher ratio values indicate a higher metallicity (Burgasser \& Kirkpatrick, 2006). Within the error bars, the thin and thick disk populations exhibit very similar behavior, indicating that the sample may not be probing a large spread ( $\gtrsim 1$ dex) in metallicity. 
spread in metallicity.

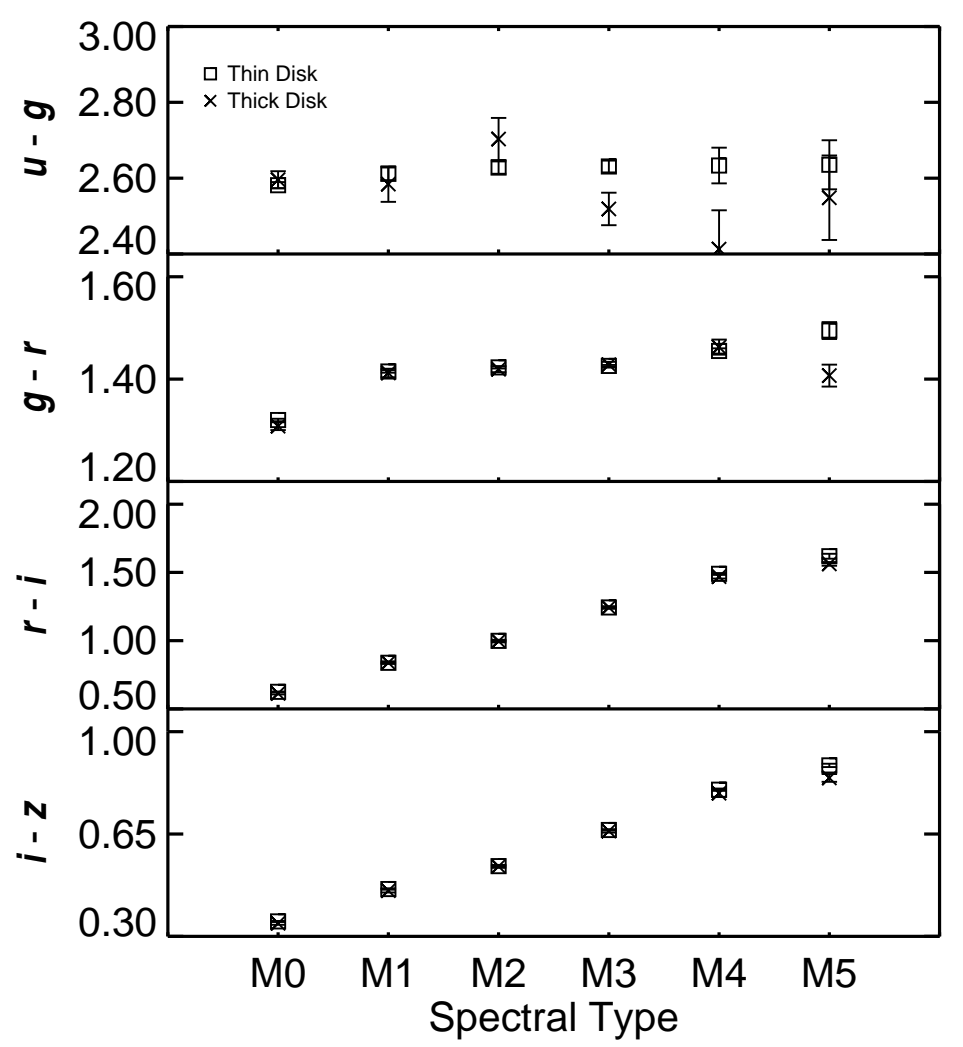

Figure 3.11 The ugriz colors as a function of spectral type for the thin (open squares) and thick (crosses) disk populations. The colors of both populations agree within the errors, suggesting that the M dwarfs in SLoMaSS do not cover a large metallicity range.

\section{Chromospheric Activity}

Finally, if the thick disk is an older system (as suggested by its hotter kinematics), it should possess a lower fraction of active stars (West et al., 2004, 2006). The chromospheric activity timescale varies with mass, such that higher mass stars lose their activity sooner (after $\sim 1$ Gyr) than their low-mass counterparts $(\sim 10$ Gyr). To observationally quantify chromospheric activity, the $\mathrm{H} \alpha$ equivalent width $(\mathrm{EW})$ is measured for each M dwarf. We employed the technique introduced in West et al. 
(2004) and described in Bochanski et al. (2007b), selecting chromospherically active and inactive stars at each spectral type. To be classified as active, a star must have an EW of $1 \AA$, and pass additional signal-to-noise and error tests described in West et al. (2004). Figure 3.12 shows the active fraction of stars as a function of spectral type for both disk populations. While most early-type M dwarfs (M0-M3) lose their activity quite rapidly, the later types (M5) possess smaller active fractions in the thick disk, and exhibit the expected behavior of older systems. However, these results are only suggestive, as populations older than $\sim 4$ Gyr require M dwarfs with types later than M5 in order to be effective probes of the age (West et al., 2007).

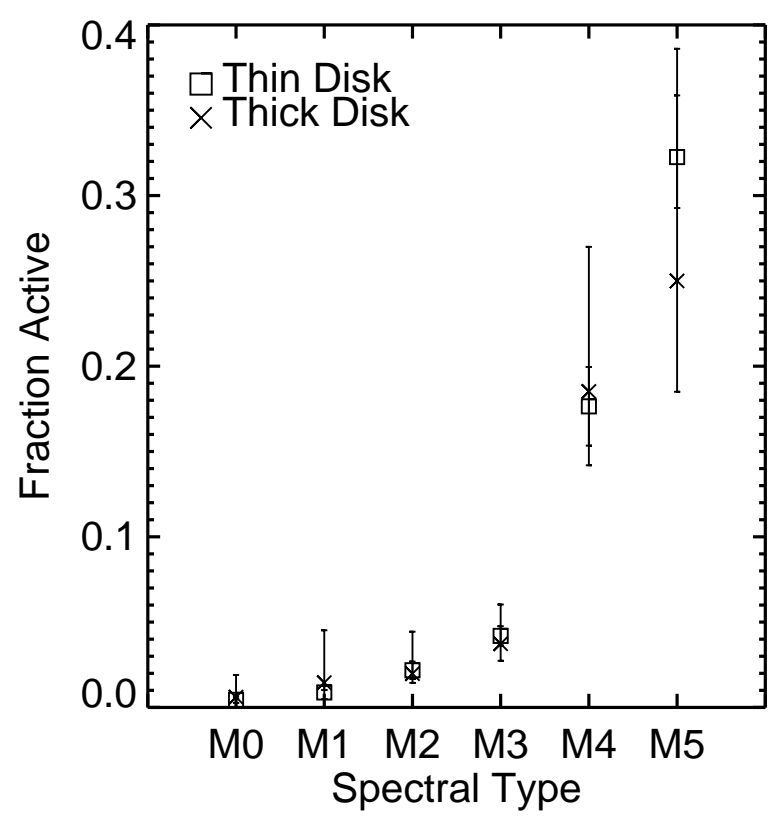

Figure 3.12 Shown is active fraction of stars as a function of spectral type for the thin (open squares) and thick (crosses) disk populations. An older population would show a lower active fraction, as suggested by the thick disk stars in the M5 bin.

\subsection{Conclusions}

The Milky Way (along with other spiral galaxies) is evidently a composite of a few major smooth components (the thin and thick disks and the halo) and many smaller 
structures, such as the tidal debris streams from the Sagittarius dwarf galaxy. Using spectra, proper motions and photometry along one Galactic sight-line, we studied the kinematic and structural distributions of the smooth thin and thick disks as a function of vertical distance from the Plane. We fit two-component Gaussians to the $U V W$ distributions as a function of height, placing new constraints on the thin and thick disk velocity dispersions.

This sample was then employed to test the predictions of current Galactic models. The Besançon model was chosen for comparison, since it is widely accepted as a standard and models the kinematics of stellar populations. We generated a suite of 25 models, and each model was sampled to be consistent with the colors and distance distribution of SLoMaSS. The bulk kinematic properties of the data and model were compared. They agree to $\sim 10 \mathrm{~km} \mathrm{~s}^{-1}$, placing a limit on the accuracy of model predictions. However, $\sigma_{W}$ is poorly fit by the model, suggesting that further investigation into modeling the kinematics of the thin and thick disks is necessary. In particular, the age-velocity dispersion plays an important role in the kinematic predictions, and a more exact definition of this relation is required.

SLoMaSS was divided kinematically, assigning membership of each star to either the thin or thick disk. We inspected the two populations as functions of spectral type, searching for observable differences in the metallicity, colors and chromospheric activity. While there was little observed difference between the metallicity and color distributions, the activity fraction distribution suggests that the thick disk displays an activity level consistent with an older population, although this result is purely suggestive and needs to be re-examined with later spectral types. The lack of a strong observable signal may have several causes. Primarily, kinematic separation of populations is not perfect, as stars with extreme kinematics in one population (i.e. the thin disk) can masquerade as members of the other population. This would dilute observable differences, and in our case, the more numerous thin disk population may be polluting the thick disk sample, despite our best efforts to minimize this effect. 
Secondly, the intrinsic properties (i.e. metallicity) of the stars that compose the thin and thick disks are drawn from overlapping distributions. Stars that are at the edges of these distributions would also blur the distinction among observable properties, clouding the best efforts of the observer. Further investigations using stellar tracers along the entire main sequence should alleviate some of these issues.

The authors would like to thank the referee, Neill Reid, whose comments greatly improved the scope of the analysis. We also gratefully acknowledge the support of NSF grants AST02-05875 and AST06-07644 and NASA ADP grant NAG5-13111.

Funding for the SDSS and SDSS-II has been provided by the Alfred P. Sloan Foundation, the Participating Institutions, the National Science Foundation, the U.S. Department of Energy, the National Aeronautics and Space Administration, the Japanese Monbukagakusho, the Max Planck Society, and the Higher Education Funding Council for England. The SDSS Web Site is http://www.sdss.org/.

The SDSS is managed by the Astrophysical Research Consortium for the Participating Institutions. The Participating Institutions are the American Museum of Natural History, Astrophysical Institute Potsdam, University of Basel, University of Cambridge, Case Western Reserve University, University of Chicago, Drexel University, Fermilab, the Institute for Advanced Study, the Japan Participation Group, Johns Hopkins University, the Joint Institute for Nuclear Astrophysics, the Kavli Institute for Particle Astrophysics and Cosmology, the Korean Scientist Group, the Chinese Academy of Sciences (LAMOST), Los Alamos National Laboratory, the MaxPlanck-Institute for Astronomy (MPIA), the Max-Planck-Institute for Astrophysics (MPA), New Mexico State University, Ohio State University, University of Pittsburgh, University of Portsmouth, Princeton University, the United States Naval Observatory, and the University of Washington. 
Table 3.1. Previous Low-Mass Stellar Kinematic Survey Results

\begin{tabular}{lcrrrrl}
\hline \hline \multicolumn{1}{c}{ Study } & $\begin{array}{c}\text { Sample } \\
\text { Size }\end{array}$ & $\begin{array}{c}\text { Distance } \\
\text { Limit (pc) }\end{array}$ & $\sigma_{U}{ }^{\mathrm{a}}$ & $\sigma_{V}$ & $\sigma_{W}$ & Comments \\
\hline Vyssotsky (1956); Reid et al. (1995a) & 368 & $\sim 300$ & 39 & 26 & 23 & McCormick stars in PMSU survey \\
Wielen (1977b) & 516 & $\sim 300$ & 39 & 23 & 20 & McCormick stars \\
Reid et al. (1995a) & 514 & 25 & 43 & 31 & 25 & PMSU I volume-complete sample \\
Ratnatunga \& Upgren (1997) & 773 & $\sim 300$ & 30.6 & 18.5 & 7.4 & McCormick stars \\
Reid et al. (2002) & 436 & 25 & 37.9 & 26.1 & 20.5 & PMSU IV volume-complete sample \\
& & 25 & 34 & 18 & 16 & PMSU IV volume-complete sample, core \\
Bochanski et al. (2005) & 100 & 35 & 21 & 22 & Non-active stars, core \\
& 15 & 100 & 19 & 15 & 16 & Active stars, core \\
This study & 70 & 100 & 28.4 & 21.3 & 19.2 & $|z|<100$ pc \\
& 4300 & 500 & 25.7 & 20.9 & 14.1 & $|z|<100$ pc, core \\
& & 500 & 34.1 & 24.6 & 24.8 & $|z|<500$ pc \\
& 6893 & 1000 & 44.1 & 36.9 & 27.9 & $|z|<500$ pc, core \\
& & 1000 & 37.4 & 27.2 & 23.6 & $|z|<1000$ pc \\
& 7398 & 1600 & 46.6 & 42.2 & 29.3 & Total Sample core \\
& & 1600 & 38.5 & 28.6 & 24.4 & Total Sample, core \\
\hline
\end{tabular}

a The velocity dispersions in $U, V$, and $W$ are measured in $\mathrm{km} \mathrm{s}^{-1}$. 


\section{Chapter 4}

\section{ABSOLUTE MAGNITUDES \& COLORS OF NEARBY LOW-MASS DWARFS}

\subsection{Introduction}

Determining the distance to an astronomical object is a fundamental observational measurement. For nearby stars, trigonometric parallax provides a direct method of computing their distances. Earth's orbit around the Sun induces an apparent angular motion of a nearby star against a grid of background objects, with this parallactic angle inversely related to the star's separation from the Sun. The largest trigonometric parallax survey to date was conducted by the Hipparcos satellite (ESA, 1997; van Leeuwen, 2007), which measured parallaxes for all stars brighter than $V \sim 8$ mag, including many $\mathrm{M}$ dwarfs down to $V \sim 12$ mag. The Hipparcos observations anchor nearby kinematic studies (e.g. Binney et al., 2000), Cepheid distances (Feast \& Catchpole, 1997) and volume-complete surveys (Reid et al., 2002).

Unfortunately, trigonometric parallaxes are not available for many fainter stars, including the overwhelming majority of the low-mass dwarfs observed by SDSS. Thus, other methods must be employed to estimate a star's distance. Two common techniques are known as photometric parallax and spectroscopic parallax, which employ a star's color and spectral type, respectively. While these quantities are not formally related to measuring a parallactic angle, they do yield an estimate of a star's distance. These methods are calibrated with sources of known absolute magnitude (nearby trig parallax stars, clusters, etc.) and relations are fit to their color (or spectral type) absolute magnitude locus. Thus, the color (or spectral type) of a star can be used to estimate its absolute magnitude, and in turn, its distance, by the well-known distance 
modulus:

$$
d=10^{\left(m_{\lambda, 1}-M_{\lambda, 1}\left(m_{\lambda, 1}-m_{\lambda, 2}\right)-5\right) / 5}
$$

where $d$ is the distance, $m_{\lambda, 1}$ and $M_{\lambda, 1}$ are the apparent and absolute magnitudes in one filter, and $m_{\lambda, 1}-m_{\lambda, 2}$ is the color in two filters, which is used to calculate the absolute magnitude, $M_{\lambda, 1}\left(m_{\lambda, 1}-m_{\lambda, 2}\right)$.

There have been numerous photometric parallax relations ${ }^{1}$ constructed for lowmass stars observed by SDSS (Hawley et al., 2002; Williams et al., 2002; West et al., 2005; Jurić et al., 2008; Golimowski et al., 2009; Sesar et al., 2008). As shown in Figures 4.1 and 4.5 , there is a significant spread ( 0.5 mags $)$ among the relations. To address this situation, ugriz photometry of a large sample of nearby stars with wellmeasured trig parallaxes is required. Fortunately, Golimowski et al. (2009) acquired such a sample and kindly provided me with their data prior to publication. The resulting CMRs form the lynch pin of this investigation into the luminosity function and mass function, allowing for the estimation of the absolute magnitude and distance to every low-mass star in the SDSS photometric database. In $\S 4.2$, I describe the selection of the nearby trig parallax stars (comprised mostly of stars from Golimowski et al., 2009) and their photometric observations. Analysis of the data follows in $\S 4.3$ and the resulting color-absolute magnitude relations are discussed in $\S 4.4$. My conclusions are found in $\S 4.5$.

\subsection{Sample Selection \& Observations}

\subsubsection{Photometric Telescope Observations}

The objective of the nearby star survey of Golimowski et al. (2009) was the creation of precise, well-sampled photometric parallax relations in SDSS ugriz and 2MASS

\footnotetext{
${ }^{1}$ Photometric parallax relations are often referred to as "color-magnitude relations" (CMRs). I will use both names interchangeably throughout this dissertation.
} 
$J H K$ filters. To be included in the initial target list, stars had to possess the colors of low-mass dwarfs and have a precise trig parallax. The majority of the nearby target stars were drawn from the extensive catalog of the Research Consortium on Nearby Stars (RECONS) team (e.g., Henry et al., 1994; Kirkpatrick et al., 1995; Henry et al., 2004). Most of the stars selected from the RECONS sample are closer than 10 pc, with good parallactic precision $>90 \%\left(\sigma_{\pi} / \pi \lesssim 0.1\right)$. In addition to the targets from RECONS, the nearby sample included K dwarfs from the Luyten (1979) and Giclas et al. (1971) proper motion studies. Parallax measurements for these additional stars were obtained from the Hipparcos (ESA, 1997) or General Catalogue of Trigonometric Stellar Parallaxes (the "Yale" catalog; van Altena et al., 1995) surveys.

Near-infrared $J H K$ photometry was secured from the 2MASS Point Source Cata$\log$ (Cutri et al., 2003). Acquiring ugriz photometry proved more problematic. Since typical SDSS photometry saturates near $r \sim 15$, most of the nearby stars were too bright to be directly imaged with the $2.5 \mathrm{~m}$ telescope. Instead, the $0.5 \mathrm{~m}$ Photometric Telescope (PT) was used to obtain $(\text { ugriz) })^{\prime}$ photometry $^{2}$ of these stars. The PT is employed every night the $2.5 \mathrm{~m}$ telescope is in use, observing patches of the nightly footprint to determine the photometric solution of the night and calibrating zeropoint of the 2.5m observations (Smith et al., 2002; Tucker et al., 2006). Golimowski et al. (2009) obtained (ugriz)' PT photometry of the parallax sample over 20 nights for 268 low-mass stars. To produce a CMR for $2.5 \mathrm{~m}$ observations, this "primed" photometry needed to be transformed to the native "unprimed" $2.5 \mathrm{~m}$ system. As shown conclusively in Davenport et al. (2007), the published transformations (Tucker et al., 2006) between these two systems are not appropriate for red, low-mass dwarfs. Thus, both the typical transformations (Tucker et al., 2006) and the Davenport et al. (2007) corrections were applied to the nearby star photometry.

To determine a reliable photometric parallax relation, both photometry and par-

\footnotetext{
${ }^{2}(u g r i z)^{\prime}$ refers to $u^{\prime} g^{\prime} r^{\prime} i^{\prime} z^{\prime}$ photometry, which is defined by the standard stars of Smith et al. (2002) observed by the USNO $1 \mathrm{~m}$ telescope.
} 
allaxes must be accurate. The following criteria were imposed on the sample. First, stars with large photometric errors ( $\sigma>0.1$ mags) in the griz bands were removed. This constraint was not placed on the $u$ band, as many of the red dwarfs in this sample do not produce appreciable levels of flux in that filter. Note that the median uncertainties, as reported in Table 4.1 are much smaller than 0.1 mags. This cut removed 11 stars from the initial sample. Next, high signal-to-noise 2MASS photometry was selected, by choosing stars with their ph_qual flag equal to 'AAA'. This flag corresponds to a signal-to-noise ratio $>10$ and photometric uncertainties $<0.1$ mags in the $J H K$ bands. This cut removed 43 stars from the original sample. Finally, a limit on parallactic accuracy of $\sigma_{\pi} / \pi<0.10$ was enforced. This cut removed an additional 25 stars. It also ensured that the bias introduced by a parallax-limited sample, described by Lutz \& Kelker (1973) is minimized. Since many of the stars in the nearby star sample have precise parallaxes $\left(\sigma_{\pi} / \pi<0.04\right)$ the Lutz-Kelker correction is essentially negligible $(<-0.05$; Hanson, 1979). The positions, photometry and parallaxes of the final sample (after the cuts described below in §4.2.3) are listed in Table 4.1.

\subsubsection{Other Parallax Stars}

To supplement the original PT observations, the literature was searched for other lowmass stars with accurate parallaxes and ugriz and $J H K$ photometry. The studies of Dahn et al. (2002) and Vrba et al. (2004) supplemented the original sample and provided accurate parallaxes $\left(\sigma_{\pi} / \pi \lesssim 0.1\right)$ of late $\mathrm{M}$ and L dwarfs. Furthermore, I selected only Dahn et al. (2002) and Vrba et al. (2004) stars that had been observed with the SDSS $2.5 \mathrm{~m}$ telescope, providing native photometry and obviating the need for transformations between the primed and unprimed ugriz systems. Six late $\mathrm{M}$ and L dwarfs were added from these catalogs, extending the parallax sample in color from $r-i \sim 2.5$ to $r-i \sim 3.0$ and in $M_{r}$ from 16 to 20 . 


\subsubsection{Contamination}

In order to determine accurate CMRs, great care must be taken to eliminate potential contaminants. Nearby stars that do not have the colors of low-mass dwarfs (i.e.., white dwarfs) are easily excluded. Four nearby white dwarfs (Van Maanen's star, HL 4, V1201 Ori, GJ 1221) were removed, since photometric parallaxes to these objects are not relevant to this dissertation. More troubling are point sources that possess the colors of low-mass dwarfs, but have abnormal absolute magnitudes. There are three potential classes of objects that would skew our sample: low-metallicity subdwarfs, binary stars and flare stars.

Since most of the stars in this sample are nearby and bright, many have known spectral classifications. Thus, any known subdwarfs were excluded from the sample. Only one outlier remained below the main sequence, where subdwarfs would be ex-

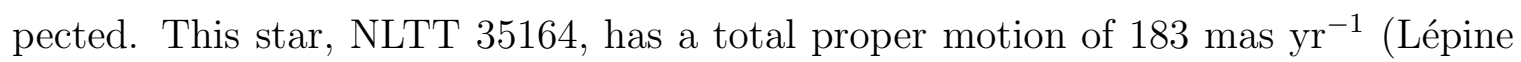
\& Shara, 2005), possibly indicating that the star is a member of an old population. This star was removed from the sample.

Binary stars are among the most troubling contaminants of the nearby star sample. An unresolved binary will have colors consistent with a low-mass dwarf, but the combined luminosity of its two components will artificially brighten the star, resulting in a smaller absolute magnitude. This effect is greatest for $\sim$ equal mass binaries, as the absolute magnitude will be underestimated by $\sim 0.75$ mags. Many unresolved, low-mass binaries likely do have mass ratios near unity (Reid \& Gizis, 1997; Burgasser et al., 2007). However, unidentified binary contaminants should be minimal in this sample. Many of these stars are nearby $(\mathrm{d}<10 \mathrm{pc})$, and thus have been studied extensively, both by radial velocity studies (e.g. Nidever et al., 2002) and high-resolution adaptive optics imaging (e.g., Beuzit et al., 2004) and IR-speckle interferometry (Henry \& McCarthy, 1990). All known binaries were removed from the sample. This is evident in Figure 4.1, where no outliers are seen far above the main 
sequence. While this did exclude some resolved pairs, it ensured that the photometry would not be skewed by pairs with small spatial separations.

Many low-mass stars possess active magnetic dynamos (e.g. West et al., 2008) and flare regularly (Lacy et al., 1976). Over the course of 20 nights of imaging, it is possible that a target star would be observed during a flare. As flares produce a rise in the blue continuum of the host star, this would be observed as anomalously blue $u-g$ and $g-r$ colors. These skewed colors, along with the enhanced luminosity produced during a flare, make these transient events undesirable for CMRs. To remove flares, we imposed a color-cut of $g-r<0.5$. This color is much bluer than the typical $g-r$ color of low-mass dwarfs ( 1.5; Bochanski et al., 2007b; Covey et al., 2007b; West et al., 2008). Thus, only bona-fide flares should be removed, leaving behind quiescent low-mass dwarfs. There were six potential flare (and blue, high-mass) contaminants.

After removing all known contaminants, bad photometry and inaccurate parallax measurements, I am confident that the remaining objects form a precise, wellsampled, unbiased sample of nearby trig parallax stars. In Figure 4.2, the ugriz color-color diagrams of the parallax stars are plotted, along with the locus of stars from the SDSS photometric sample (see Chapter 5). The stars track the underlying locus, indicating no major systematic bias in our selection. The 86 stars in the final trig parallax sample after the cuts described above are listed in Table 4.1. 


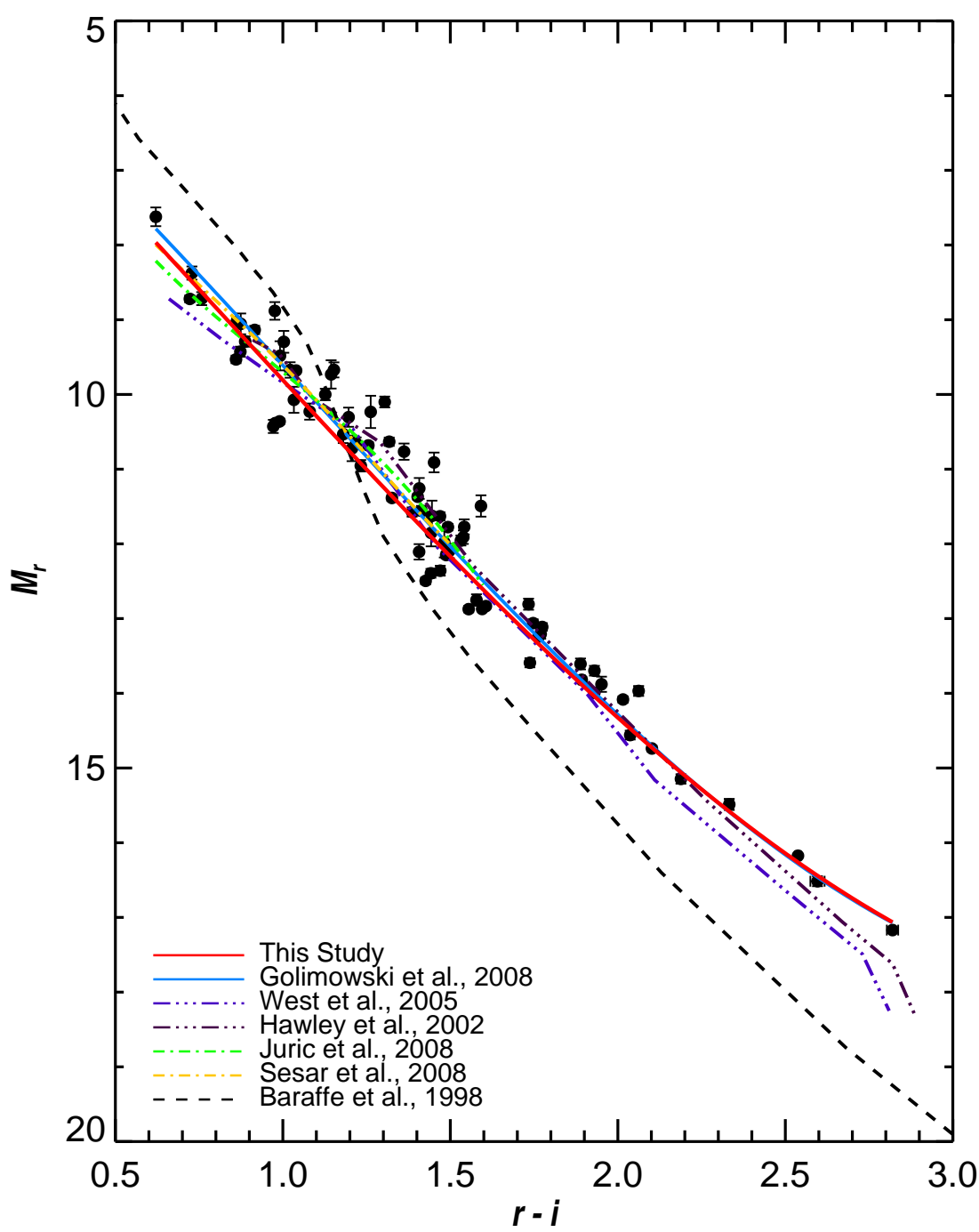

Figure $4.1 M_{r}$ vs. $r-i$ CMD. The parallax stars listed in Table 4.1 are shown as filled circles and the best fit line from Table 4.2 is the solid red line. Other existing parallax relations are plotted for comparison: Hawley et al. (2002) (dark dash-dot line), West et al. (2005) (purple dash-dot line), Jurić et al. (2008) (green dash-dot line), Sesar et al. (2008) (yellow dash-dot line), Golimowski et al. (2009) (solid blue line). The original Hawley et al. (2002) and West et al. (2005) relations have been transformed using data from their color/absolute-magnitude tables. In addition, the 5 Gyr isochrone from the Baraffe et al. (1998) models appears as the dashed line. The differences between the CMR from this study and the others on this plot are shown in Figure 4.5. 

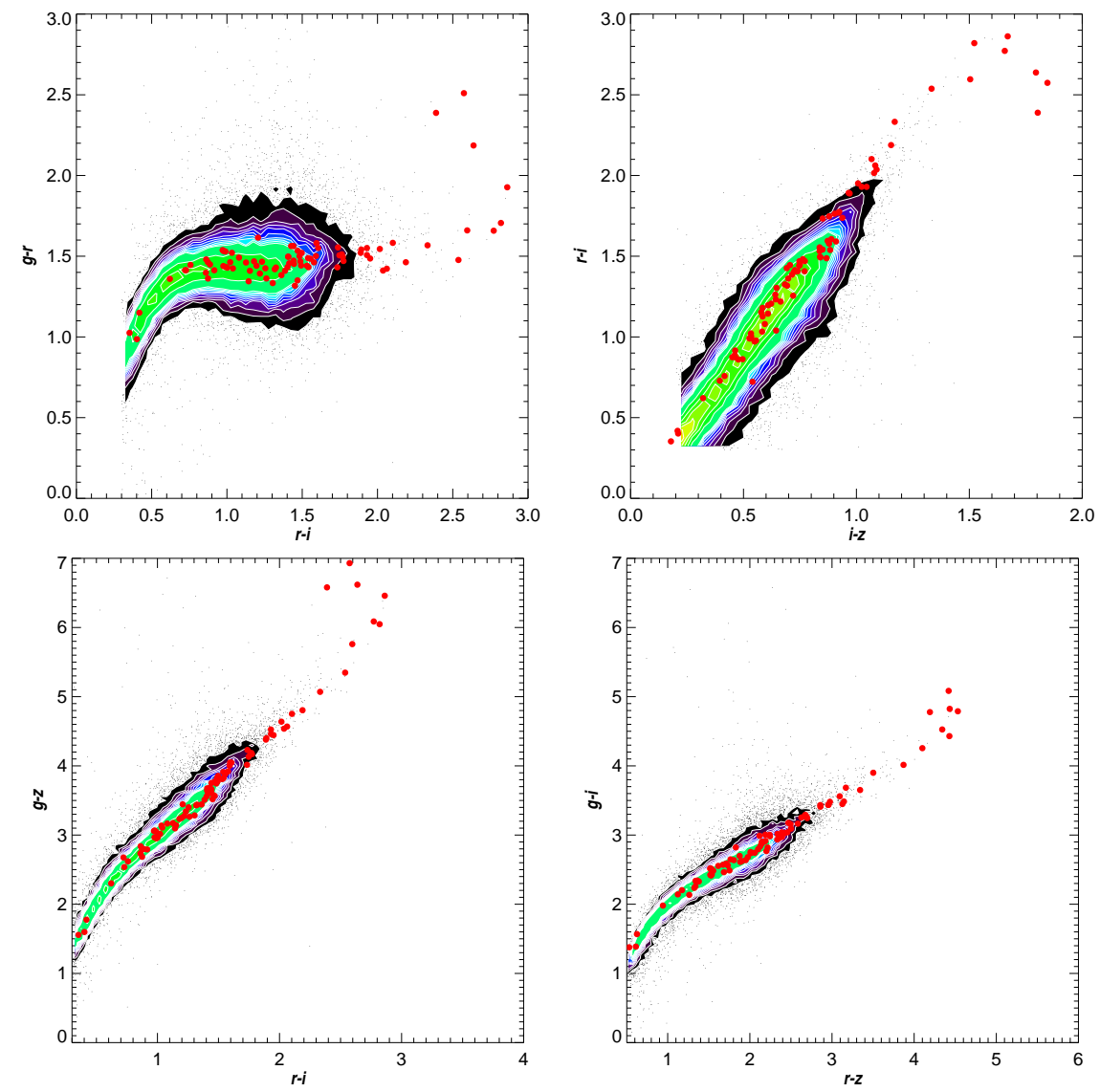

Figure 4.2 Color-color diagrams of the SDSS photometric sample (contours) with the trig parallax stars overplotted as filled red circles. The contours represent $0.2 \%$ of the entire sample, with contours increasing every 10 stars per 0.05 color-color bin. The typical color error bars of the parallax sample stars are smaller than the plot symbols. These diagrams demonstrate that the nearby star sample is drawn from the densest regions of the low-mass stellar locus and extend beyond the SDSS color-color space. 
Table 4.1. ugrizJHK Photometry of Trig. Parallax Stars

\begin{tabular}{|c|c|c|c|c|c|c|c|c|c|c|c|c|c|c|c|c|c|c|c|c|}
\hline Name & $\alpha(\mathrm{J} 2000)$ & $\delta(\mathrm{J} 2000)$ & $u$ & $g$ & $r$ & $i$ & $z$ & $\sigma_{u}$ & $\sigma_{g}$ & $\sigma_{r}$ & $\sigma_{i}$ & $\sigma_{z}$ & $J$ & $H$ & $K$ & $\sigma_{J}$ & $\sigma_{H}$ & $\sigma_{K}$ & $\pi(\prime \prime)$ & $\sigma_{\pi}$ \\
\hline GJ 1002 & 1.6797228 & -7.5385500 & 17.695 & 14.694 & 13.111 & 11.009 & 9.942 & 0.041 & 0.005 & 0.004 & 0.002 & 0.002 & 8.323 & 7.792 & 7.439 & 0.019 & 0.034 & 0.021 & 0.213000 & 0.003600 \\
\hline GJ 1025 & 15.2346544 & -4.4492310 & 16.881 & 14.256 & 12.693 & 11.251 & 10.504 & 0.034 & 0.009 & 0.007 & 0.005 & 0.006 & 9.042 & 8.485 & 8.224 & 0.032 & 0.047 & 0.021 & 0.087700 & 0.002380 \\
\hline G1 54.1 & 18.1278454 & -16.9987460 & 15.817 & 13.012 & 11.458 & 9.720 & 8.783 & 0.030 & 0.004 & 0.003 & 0.003 & 0.003 & 7.258 & 6.749 & 6.420 & 0.020 & 0.033 & 0.017 & 0.268838 & 0.007616 \\
\hline LHS 1302 & 27.7667010 & -6.1179720 & & 15.357 & 13.816 & 11.924 & $\begin{array}{r}0.958 \\
10.958\end{array}$ & & 0.015 & 0.008 & 0.004 & 0.004 & 9.413 & 8.841 & $\begin{array}{l}8.452 \\
8.552\end{array}$ & 0.021 & 0.021 & 0.022 & $\begin{array}{l}0.200000 \\
0.100780\end{array}$ & 0.001890 \\
\hline LHS 1326 & 30.5674712 & 10.3372090 & 17.654 & $\begin{array}{l}10.0381 \\
16.377\end{array}$ & $\begin{array}{l}13.010 \\
14.914\end{array}$ & $\begin{array}{l}11.924 \\
12.726\end{array}$ & $\begin{array}{l}10.570 \\
11.572\end{array}$ & 0.040 & 0.015 & 0.010 & $\begin{array}{l}0.004 \\
0.004\end{array}$ & 0.004 & $\begin{array}{l}9.410 \\
9.842\end{array}$ & $\begin{array}{l}.041 \\
9.254\end{array}$ & $\begin{array}{l}0.002 \\
8.928\end{array}$ & 0.023 & $\begin{array}{l}0.025 \\
0.025\end{array}$ & 0.025 & 0.112000 & 0.003200 \\
\hline LHS 1358 & 33.2277320 & 0.0048010 & 17.054 & 14.418 & $\begin{array}{l}14.914 \\
12.930\end{array}$ & $\begin{array}{l}12.326 \\
11.392\end{array}$ & 10.508 & 0.040 & $\begin{array}{l}0.013 \\
0.011\end{array}$ & 0.006 & $\begin{array}{l}0.004 \\
0.003\end{array}$ & $\begin{array}{l}0.004 \\
0.003\end{array}$ & $\begin{array}{l}9.842 \\
9.055\end{array}$ & $\begin{array}{l}9.254 \\
8.518\end{array}$ & $\begin{array}{l}8.928 \\
8.168\end{array}$ & $\begin{array}{l}0.023 \\
0.030\end{array}$ & $\begin{array}{l}0.025 \\
0.026\end{array}$ & $\begin{array}{l}0.025 \\
0.021\end{array}$ & $\begin{array}{l}0.112000 \\
0.062930\end{array}$ & $\begin{array}{l}0.0032600 \\
0.00200\end{array}$ \\
\hline $\begin{array}{l}\text { LHS } 1358 \\
\text { LHS } 1375\end{array}$ & 34.1245767 & $\begin{array}{r}0.0480010 \\
13.5868020\end{array}$ & & $\begin{array}{l}14.418 \\
16.715\end{array}$ & $\begin{array}{l}12.930 \\
15.148\end{array}$ & $\begin{array}{l}11.392 \\
12.815\end{array}$ & $\begin{array}{l}10.608 \\
11.646\end{array}$ & & $\begin{array}{l}0.011 \\
0.019\end{array}$ & $\begin{array}{l}0.006 \\
0.012\end{array}$ & $\begin{array}{l}0.003 \\
0.005\end{array}$ & $\begin{array}{l}0.003 \\
0.004\end{array}$ & $\begin{array}{l}9.055 \\
9.871\end{array}$ & $\begin{array}{l}8.5 \\
9.3\end{array}$ & $\begin{array}{l}8.168 \\
8.981\end{array}$ & $\begin{array}{l}0.0 \\
0.0\end{array}$ & $\begin{array}{l}0.026 \\
0.022\end{array}$ & $\begin{array}{l}0.0 \\
0.0\end{array}$ & $\begin{array}{l}0.062930 \\
0.117700\end{array}$ & $\begin{array}{l}0.002000 \\
0.004000\end{array}$ \\
\hline Gl 109 & 41.06474 & 25.52316 & 14.159 & 11.4 & 10.08 & 8.861 & 8.197 & 0.007 & 0.004 & 0.00 & 0.004 & 0.004 & 6.752 & 61 & 5.961 & & & & & \\
\hline $\mathrm{L}$ & 48.345742 & 4.7 & 17.687 & 4.876 & 13.369 & $\begin{array}{r}.001 \\
11.439\end{array}$ & 10.416 & 0.118 & 01 & 0.005 & .004 & 0.0 & .775 & 8 & .833 & $\begin{array}{l}0 \\
0\end{array}$ & & 4 & & \\
\hline GJ 1065 & 57.6843430 & $\begin{array}{r}-6.0950790 \\
\end{array}$ & 16.568 & 13.792 & 12.259 & 10.789 & 10.017 & 0.051 & 0.006 & 0.004 & 0.003 & 0.003 & 370 & $\begin{array}{l}0.2008 \\
7.998\end{array}$ & .751 & 2 & 0.057 & 0.020 & 5400 & 03200 \\
\hline Gl $169.1 \mathrm{~A}$ & 67.7987069 & 58.9766860 & & 11.939 & 10.497 & 9.004 & 8.163 & & 0.003 & 0.003 & 0.003 & 0.001 & 6.622 & 6.012 & 5.717 & 0.021 & 0.020 & $\begin{array}{l}0.021 \\
0.021\end{array}$ & .181360 & .003670 \\
\hline LHS 1723 & 75.4891313 & -6.9461280 & 15.962 & 13.097 & 11.516 & 9.921 & 9.048 & 0.019 & 0.005 & 0.003 & 0.003 & 0.003 & 7.617 & 7.065 & 6.736 & 0.032 & 0.046 & 0.024 & 0.187920 & 0.001260 \\
\hline G $191-47$ & 84.2669629 & 52.5232770 & & 10.666 & 9.680 & 9.278 & 9.067 & & 0.004 & 0.003 & 0.003 & 0.003 & 7.994 & 7.427 & 7.272 & 0.018 & 0.031 & 0.018 & 0.026120 & 0.001900 \\
\hline G $99-49$ & 90.0146333 & 2.7065150 & 14.956 & 12.226 & 10.706 & 9.209 & 8.370 & 0.013 & 0.004 & 0.003 & 0.003 & 0.003 & 6.905 & 6.308 & 6.042 & 0.020 & 0.023 & 0.023 & 0.190770 & 0.008478 \\
\hline LHS 1809 & 90.6214440 & 49.8653680 & & 15.397 & 13.847 & 11.917 & 10.872 & & 0.010 & 0.006 & 0.004 & 0.006 & 9.350 & 8.768 & 8.435 & 0.023 & 0.029 & 0.020 & 0.107700 & 0.002600 \\
\hline Gl 232 & 96.1721896 & 23.4328020 & 16.807 & 13.987 & 12.503 & 10.948 & 10.111 & 0.019 & 0.004 & 0.004 & 0.003 & 0.003 & 8.662 & 8.156 & 7.911 & 0.020 & 0.061 & 0.016 & 0.119400 & 0.002300 \\
\hline Gl 251 & 103.7040758 & 33.2680010 & 13.514 & 10.850 & 9.425 & 8.170 & 7.450 & 0.007 & 0.005 & 0.003 & 0.003 & 0.003 & $\begin{array}{l}6.004 \\
6.104\end{array}$ & 5.526 & 5.275 & 0.024 & 0.016 & 0.023 & 0.17 & 0.003585 \\
\hline GJ 1093 & 104.8 & & & 15. & 14.0 & 11.986 & 10.897 & & 0.0 & 0.0 & & & 9.1 & & & & & & & \\
\hline BL I & & & & 12. & & & $\begin{array}{r}10.098 \\
9.066\end{array}$ & & & & & & & & & & & & & \\
\hline & & & & & & & & & & & & & & & & & & & & \\
\hline & & & 15.575 & $\begin{array}{l}10.8 \\
12.8\end{array}$ & 11. & & & 0.016 & & & 0.0 & & 7.7 & 7.11 & & & & & & 0.01 \\
\hline & & & 13.573 & 1 & & & & 0.012 & & & & & & & & & & & & 226 \\
\hline GJ 11 & 12 & 26.7 & 17.042 & 15.4 & & 11.4 & 10.1 & 0.028 & & 0.0 & & & 8.2 & & 7.260 & & & & & \\
\hline & & & & 1 & & & 9.3 & & & & & & & & & & & & & \\
\hline WT & & & & & & & 11.2 & & & 0.0 & & & & & & & & & & \\
\hline Gl 4 & & & 14.259 & & & & 8. & 0.008 & & & & 0. & & 6. & & & & & & \\
\hline & & & 14.851 & 12. & 10. & & 7.9 & 0.013 & & 0.0 & 0.0 & 0.0 & 6.5 & 5.9 & 5.6 & & & & & \\
\hline & & & & 14.1 & 12.641 & 10.894 & 10.015 & & 0.0 & 0.00 & 0.0 & 0.00 & 8.488 & 7.952 & 7.6 & 0.0 & & 0.017 & & 0.002900 \\
\hline Gl 452.4 & 8.739465 & 28.7374420 & $\ldots$ & 11.285 & 9.925 & 9.304 & 8.983 & & 0.003 & 0.003 & 0.0 & 0.004 & 7.802 & 7.159 & 6.998 & 0.023 & 0.020 & 0.018 & 0.034850 & 0.002010 \\
\hline Gl 455 & 180.5750314 & 28.5870040 & & 13.882 & 12.267 & 11.060 & 10.438 & & 0.004 & 0.003 & 0.003 & 0.003 & 9.132 & 8.607 & 8.393 & 0.018 & 0.020 & 0.017 & 0.049400 & 0.003900 \\
\hline GJ 1156 & 184.7470654 & 11.1261840 & 17.227 & 14.720 & 13.175 & 11.160 & 10.081 & 0.085 & 0.008 & 0.003 & 0.002 & 0.002 & 8.525 & 7.880 & 7.570 & 0.027 & 0.029 & 0.031 & 0.152900 & 0.003000 \\
\hline Gl 46 & 185.7 & 64.03 & & 12.3 & 11.017 & 9.873 & $\begin{array}{r}9.001 \\
9.264\end{array}$ & & $\begin{array}{l}0.002 \\
0.002\end{array}$ & $\begin{array}{l}.0002 \\
0.002\end{array}$ & 0.0 & 0.006 & $\begin{array}{l}7.020 \\
7.937\end{array}$ & $\begin{array}{l}1.000 \\
7.343\end{array}$ & 7.122 & 0.029 & 0.020 & 0.018 & $\begin{array}{l}0.1055696 \\
0.056\end{array}$ & 0.004858 \\
\hline GJ $1159 \mathrm{~A}$ & 187.3091539 & 53.5458010 & $\ldots$ & 14.9 & 13.6 & $\begin{array}{r}5.170 \\
12.171\end{array}$ & 11.42 & & 0.0 & $\begin{array}{l}0.003 \\
0.003\end{array}$ & 0.0 & 0.003 & 9.983 & 9.499 & 9.223 & 0.023 & 0.021 & 0.014 & $\begin{array}{l}0.039900 \\
0.03900\end{array}$ & $\begin{array}{l}0.0040000 \\
0.001000\end{array}$ \\
\hline LHS 2633 & 191.7537522 & 46.6258550 & $\cdots$ & 12.605 & 11.195 & 10.043 & 9.464 & & 0.003 & 0.002 & 0.002 & 0.002 & 8.104 & 7.472 & 7.223 & 0.020 & 0.024 & 0.017 & 0.049890 & 0.002320 \\
\hline
\end{tabular}


Table 4.1-Continued

\begin{tabular}{|c|c|c|c|c|c|c|c|c|c|c|c|c|c|c|c|c|c|c|c|c|}
\hline Name & $\alpha(\mathrm{J} 2000)$ & $\delta(\mathrm{J} 2000)$ & $u$ & $g$ & $r$ & $i$ & $z$ & $\sigma_{u}$ & $\sigma_{g}$ & $\sigma_{r}$ & $\sigma_{i}$ & $\sigma_{z}$ & $J$ & $H$ & K & $\sigma_{J}$ & $\sigma_{H}$ & $\sigma_{K}$ & $\pi(\prime \prime)$ & $\sigma_{\pi}$ \\
\hline Gl 493.1 & 195.1393252 & 5.6856280 & 16.952 & 14.267 & 12.770 & 11.000 & 10.080 & 0.064 & 0.005 & 0.002 & 0.002 & 0.002 & 8.553 & 7.966 & 7.660 & 0.035 & 0.047 & 0.031 & 0.123100 & 0.003500 \\
\hline Gl 514 & 202.4994806 & 10.3767900 & 12.565 & 9.912 & 8.431 & 7.569 & 7.073 & 0.009 & 0.003 & 0.002 & 0.003 & 0.077 & 5.902 & 5.300 & 5.036 & 0.018 & 0.033 & 0.027 & 0.132570 & 0.002963 \\
\hline G1 521 & 204.8504639 & 46.1866870 & & 11.029 & 9.667 & 8.792 & 8.342 & & 0.002 & 0.002 & 0.002 & 0.002 & 7.054 & 6.513 & 6.284 & 0.018 & 0.036 & 0.017 & 0.075956 & 0.004872 \\
\hline LHS 2784 & 205.6801701 & 33.2897420 & 15.578 & 12.876 & 11.423 & 9.978 & 9.273 & 0.009 & 0.004 & 0.003 & 0.003 & 0.004 & 7.787 & 7.212 & 6.982 & 0.020 & 0.024 & 0.020 & 0.110459 & 0.010380 \\
\hline LHS 2884 & 213.8205533 & 45.0147020 & & 12.672 & 11.310 & 10.048 & 9.407 & & 0.003 & 0.002 & 0.002 & 0.003 & 8.014 & 7.474 & 7.227 & 0.018 & 0.018 & 0.017 & 0.061300 & 0.006100 \\
\hline Gl 552 & 217.3733295 & 15.5331450 & & 11.519 & 10.090 & 9.088 & 8.553 & & 0.003 & 0.002 & 0.003 & 0.002 & 7.229 & 6.605 & 6.393 & 0.021 & 0.031 & 0.018 & 0.069799 & 0.004702 \\
\hline G1 555 & 218.5703067 & -12.5192930 & 14.825 & 12.159 & 10.727 & 9.186 & $\begin{array}{l}8.0335 \\
8.335\end{array}$ & 0.018 & 0.007 & 0.002 & 0.003 & 0.004 & 6.838 & $\begin{array}{l}6.262 \\
6.262\end{array}$ & $\begin{array}{l}5.039 \\
5.939\end{array}$ & 0.019 & 0.044 & 0.034 & 0.162863 & 0.007545 \\
\hline LHS 3018 & & & & 11.7 & & & 8000 & & & & & & 7.7 & & & & & & & \\
\hline c & 2 & - & 14.136 & $.4 \quad$ & & 8.7 & 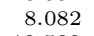 & 0.011 & & & & & & & & & & & & \\
\hline & & 17.46 & & .6 & 13.17 & 1.4 & .58 & & & & & & & & & & & & & \\
\hline IS 308 & & 2 & $\ldots$ & 1 & 3.695 & 2.104 & 11.194 & & 0.007 & 0.00 & 0.002 & 0.005 & .673 & & 820 & & & & & \\
\hline Gl 609 & 0.7117099 & 20.5887690 & $\ldots$ & 3.403 & 11.962 & 10.431 & 9.594 & .. & 0.004 & 0.003 & 0.004 & 0.005 & 8.132 & 7.648 & 7.369 & 0.020 & .023 & & .100300 & \\
\hline G $180-45$ & 3.9786706 & 35.8210940 & & 10.312 & 9.162 & 8.744 & 8.536 & & 0.002 & 0.004 & 0.003 & 0.006 & 7.396 & 6.854 & 6.725 & 0.019 & 0.018 & 0.020 & 0.032390 & 0.001110 \\
\hline Gl 625 & 6.3528709 & 54.3041720 & 13.730 & 10.997 & 9.468 & 8.478 & 7.951 & 0.011 & 0.002 & 0.002 & 0.004 & 0.003 & 6.608 & 6.063 & 5.833 & 0.020 & 0.018 & 0.024 & 0.151793 & 0.002553 \\
\hline Gl 628 & 247.5751326 & -12.6629920 & 13.636 & .951 & 9.518 & 8.116 & 7.393 & 0.007 & 0.002 & 0.001 & 0.002 & 0.003 & 5.950 & 5.373 & 5.075 & 0.024 & 0.040 & 0.024 & 0.236010 & 0.004257 \\
\hline LTT 14949 & 250.2036846 & 36.3166380 & & .356 & 10.922 & 9.930 & 9.403 & & 0.004 & 0.003 & 0.003 & 0.003 & 8.069 & 7.416 & 7.204 & 0.021 & 0.017 & 0.020 & 0.051880 & 0.004660 \\
\hline Gl 643 & 253.8547538 & -8.3229440 & 15.423 & .620 & 11.123 & 9.717 & 8.977 & 0.021 & 0.005 & 0.0 & 0.002 & 0.0 & 7.555 & 7.0 & 6.724 & & 0.0 & & & 0.007524 \\
\hline GJ 1207 & 2 & -4.3490440 & 15.862 & 7 & .686 & 10.20 & 9.441 & 0.015 & 0.0 & 0.0 & 0.00 & & 7.971 & 7.4 & 7.120 & & 0. & & & \\
\hline LHS 3 & 2 & 51.40 & 17.327 & 1 & 1 & 11.23 & 10.314 & 0.067 & & & & & 8.7 & & 7.9 & & & & & \\
\hline GJ 12 & 256 . & 16 & & & & 10.5 & 9.9 & 0.001 & & & & & 8.5 & & 7.7 & & & & & \\
\hline Gl 65 & 256 & 21.5 & & & 11.0 & 9.8 & & & & & & & 7.8 & & 7.0 & & & & & \\
\hline & & & & & 11.0 & & & & & & & & & & & & & & & \\
\hline & & & & & & & & 0.006 & & & & & & & & & & & & \\
\hline & & & 13.101 & & & & & 0.007 & & & & & & & & & & & & \\
\hline & & 43.3 & & & & & & & & & & & & & & & & & & \\
\hline & & & & & 14.2 & 12.3 & 11.3 & & & & & & & & & & & & & \\
\hline & & & 12.858 & & & & & 0.01 & & & & & & & & & & & & \\
\hline & & & 17.334 & & & 11. & & 0.085 & & & & & & & & & & & & \\
\hline & & & & 13. & 12.4 & 11. & 10.3 & & & & & & & & & & & & & \\
\hline & & & 13.151 & 1 & 9.1 & 7.7 & 7.1 & 0.007 & & & 0.0 & & 5.7 & & 5.0 & & 0.0 & 0.0 & 5056 & 0.005310 \\
\hline & & -23.8 & 14.157 & 11.4 & 9.8 & 8.4 & 7.754 & 0.0 & 0.0 & & 0.0 & & 6.222 & 5.655 & 5.370 & 0.0 & 0.0 & 0.016 & 0.336899 & 0.007014 \\
\hline Gl $745 \mathrm{~A}$ & 286.7 & 20.8877820 & 14.356 & 11.675 & 10.137 & 9.165 & 8.616 & 0.007 & 0.003 & 0.0 & 0.003 & 0.004 & 7.295 & 6.726 & 6.521 & 0.020 & 0.0 & 0.021 & 0.115082 & 0.004646 \\
\hline Gl 745 & 286.8050672 & 20.8767580 & 14.358 & 11.668 & 10.136 & 9.159 & 8.603 & 0.007 & 0.003 & 0.004 & 0.003 & 0.004 & 7.278 & 6.752 & 6.517 & 0.021 & 0.051 & 0.023 & 0.112820 & 0.002410 \\
\hline G $207-22$ & 288.1226728 & 35.5644130 & & 12.906 & 11.415 & 10.335 & 9.740 & & 0.002 & 0.002 & 0.002 & 0.002 & 8.399 & 7.824 & 7.606 & 0.024 & 0.024 & 0.017 & 0.058300 & 0.002900 \\
\hline
\end{tabular}




\subsection{Analysis}

Using the parallaxes and photometry in $\S 4.1$, multiple color-absolute magnitude diagrams (CMDs) in the ugriz and $J H K$ bandpasses were constructed. The CMDs were individually inspected, fitting the main sequence with linear, 2nd, 3rd, and 4th order polynomials. Piecewise functions were also tested, placing discontinuities by eye along the main sequence. There is extensive discussion in the literature of a "break" in the main sequence near spectral type M4 (or $I-J \sim 1.5$, Hawley et al., 1996; Reid \& Gizis, 1997; Reid \& Cruz, 2002; Reid \& Hawley, 2005), but a sharp change in the slope of the main sequence was not strictly enforced. Certain colors, such as $V-I$ show evidence of a break (Fig. 10 of Reid \& Cruz, 2002), while other colors, such as $V-K$, do not (Fig. 9 of Reid \& Cruz, 2002). Thus, breaks were placed where appropriate. Finally, the rms scatter about the fit for each CMD was computed and the relation that produced the smallest scatter for each color-absolute magnitude combination was adopted.

\subsection{Results}

The resulting CMRs and their rms scatter are listed in Table 4.2. Below, the structure along the main sequence observed in some CMDs is explored and the CMRs are compared to previous relations. Finally, absolute-magnitude transformations are introduced and their importance in determining the luminosity function of low-mass stars is discussed.

\subsubsection{Color-Absolute Magnitude Relations}

I present three different CMRs: $\left(M_{r}, r-i\right),\left(M_{r}, r-z\right)$ and $\left(M_{r}, i-z\right)$. As these stars are cool and red, the $r$ band was selected for absolute magnitude as it receives significant flux from all late-type stars. Colors with a long wavelength baseline and

small residual $\mathrm{rms}$ scatter $(\sigma \lesssim 0.40 \mathrm{mag})$ were adopted. The adopted photometric 
Table 4.1-Continued

\begin{tabular}{|c|c|c|c|c|c|c|c|c|c|c|c|c|c|c|c|c|c|c|c|c|}
\hline Name & $\alpha(\mathrm{J} 2000)$ & $\delta(\mathrm{J} 2000)$ & $u$ & $g$ & $r$ & $i$ & $z$ & $\sigma_{u}$ & $\sigma_{g}$ & $\sigma_{r}$ & $\sigma_{i}$ & $\sigma_{z}$ & $J$ & $H$ & $K$ & $\sigma_{J}$ & $\sigma_{H}$ & $\sigma_{K}$ & $\pi(\prime \prime)$ & $\sigma_{\pi}$ \\
\hline Gl $752 \mathrm{~A}$ & 289.2299675 & 5.1684700 & 12.568 & 9.953 & 8.529 & 7.490 & 6.845 & 0.006 & 0.002 & 0.005 & 0.002 & 0.003 & 5.583 & 4.929 & 4.673 & 0.030 & 0.027 & 0.020 & 0.171005 & 0.001464 \\
\hline GJ 1235 & 290.4110401 & 20.8671760 & & 14.225 & 12.763 & 11.185 & 10.306 & & 0.008 & 0.004 & 0.003 & 0.003 & 8.796 & 8.219 & 7.935 & 0.020 & 0.036 & 0.016 & 0.100100 & 0.003500 \\
\hline GJ 1253 & 306.5222953 & 58.5732360 & 17.938 & 14.985 & 13.464 & 11.576 & 10.606 & 0.125 & 0.008 & 0.004 & 0.003 & 0.003 & 9.029 & 8.483 & 8.095 & 0.039 & 0.073 & 0.021 & 0.107500 & 0.003600 \\
\hline LP $816-60$ & 313.1374934 & -16.9745330 & 14.899 & 12.329 & 10.861 & 9.374 & 8.506 & 0.040 & 0.005 & 0.002 & 0.004 & 0.005 & 7.090 & 6.517 & 6.199 & 0.023 & 0.044 & 0.021 & 0.182150 & 0.003680 \\
\hline Gl 809 & 3.3325319 & 62.1539430 & 12.063 & 9.393 & 7.980 & 7.258 & 6.718 & 0.004 & 0.002 & 0.003 & 0.002 & 0.00 & 5.429 & 4.9 & 4.618 & 0.0 & 0.059 & 0.024 & 0.141580 & 0.003090 \\
\hline LHS 3713 & 7.0637161 & 27.9279640 & & 12.913 & 11.392 & 10.359 & 9.776 & & 0.003 & 0.002 & 0.002 & 0.003 & 8.508 & 7.929 & 7.686 & 0.018 & 0.029 & 0.016 & 0.054800 & 0.004400 \\
\hline Gl 849 & 2.4184864 & -4.6407740 & 13.764 & 11.157 & 9.824 & 8.520 & 7.873 & 0.009 & 0.001 & 0.001 & 0.001 & 0.002 & 6.510 & 5.899 & 5.594 & 0.024 & 0.044 & 0.017 & 0.114353 & 0.003818 \\
\hline Gl $127-35$ & 337.1915816 & 18.9316980 & 14.239 & 11.569 & 10.155 & 9.427 & 9.032 & 0.017 & 0.002 & 0.001 & 0.002 & 0.002 & 7.819 & 7.172 & 6.978 & 0.027 & 0.021 & 0.017 & 0.044340 & 0.001810 \\
\hline Gl 867B & 9.6886582 & -20.6140200 & 14.950 & 12.464 & 10.967 & 9.560 & 8.789 & 0.056 & 0.006 & 0.004 & 0.004 & 0.006 & 7.344 & 6.817 & 6.491 & 0.024 & 0.057 & 0.016 & 0.115100 & 0.007400 \\
\hline Gl $867 \mathrm{AC}$ & 39.6899703 & -20.6207630 & 12.5 & 10.018 & 8.579 & 7.603 & 7.052 & 0.013 & 0.003 & 0.003 & 0.004 & 0.006 & 5.669 & 5.1 & 4.800 & 0.019 & 0.049 & 0.016 & 0.115686 & 0.006293 \\
\hline Gl 908 & 357.3026612 & 2.4009890 & 12.328 & 9.810 & 8.412 & 7.552 & 7.074 & 0.014 & 0.005 & 0.003 & 0.005 & 0.008 & 5.827 & 5.282 & 5.043 & 0.023 & 0.031 & 0.020 & 0.168512 & 0.003953 \\
\hline Т 832-10443 & 43.1094933 & 0.9395220 & 22.890 & 21.867 & 19.940 & 17.078 & 15.408 & 0.503 & 0.081 & 0.030 & 0.015 & 0.024 & 13.126 & 12.441 & 11.963 & 0.023 & 0.024 & 0.019 & 0.036000 & 0.000400 \\
\hline $2 \mathrm{M} \mathrm{J} 0746+20$ & 116.6767731 & 20.0088425 & 22.852 & 21.168 & 18.658 & 16.084 & 14.238 & 0.428 & 0.044 & 0.011 & 0.011 & 0.015 & 11.759 & 11.007 & 10.468 & 0.020 & 0.022 & 0.022 & 0.081900 & 0.0003 \\
\hline Т 213-2005 & 155.3637695 & 50.9179382 & 22.843 & 21.567 & 19.909 & 17.137 & 15.480 & 0.399 & 0.050 & 0.024 & 0.022 & 0.021 & 13.393 & 12.739 & 12.256 & 0.023 & 0.023 & 0.021 & 0.030100 & 0.0004 \\
\hline GJ 3693 & 178.4696350 & 6.9984298 & 22.652 & 18.958 & 17.298 & 14.702 & 13.198 & 0.433 & 0.026 & 0.015 & 0.015 & 0.020 & 11.256 & 10.660 & 10.262 & 0.022 & 0.027 & 0.022 & 0.070200 & 0.0021 \\
\hline GJ 3849 & 217.1797333 & 33.1768990 & 21.907 & 20.972 & 18.584 & 16.195 & 14.392 & 0.203 & 0.033 & 0.016 & 0.013 & 0.019 & 11.990 & 11.225 & 10.744 & 0.021 & 0.029 & 0.024 & 0.095000 & 0.0057 \\
\hline GJ 3855 & 217.6569977 & 59.7236252 & 21.190 & 18.819 & 17.113 & 14.293 & 12.771 & 0.083 & 0.019 & 0.013 & 0.011 & 0.015 & 10.790 & 10.140 & 9.788 & 0.018 & 0.020 & 0.020 & 0.103300 & 0.001300 \\
\hline $2 \mathrm{M} \mathrm{J} 1501+22$ & 225.2840576 & 22.8338356 & 23.313 & 20.909 & 18.723 & 16.085 & 14.290 & 0.642 & 0.040 & 0.014 & 0.016 & 0.017 & 11.866 & 11.181 & 10.706 & 0.022 & 0.030 & 0.024 & 0.094400 & 0.000600 \\
\hline
\end{tabular}

Note. - Some stellar names were abbreviated. $\mathrm{T}=\mathrm{TVLM}, 2 \mathrm{M}=2 \mathrm{MASS}$ 
Table 4.2. Color- Absolute Magnitude Relations in the ugriz system

\begin{tabular}{cccc}
\hline \hline Abs. Mag. & Color & Best Fit & Scatter \\
\hline$M_{r}$ & $0.50<r-z<4.53$ & $5.190+2.474(r-z)+0.4340(r-z)^{2}-0.08635(r-z)^{3}$ & 0.394 \\
$M_{r}$ & $0.62<r-i<2.82$ & $5.025-4.548(r-i)+0.4175(r-i)^{2}-0.18315(r-i)^{3}$ & 0.403 \\
$M_{r}$ & $0.32<i-z<1.85$ & $4.748+8.275(i-z)+2.2789(g-i)^{2}-1.5337(i-z)^{3}$ & 0.481 \\
\hline
\end{tabular}

parallaxes did not include any discontinuities, although I note a slight increase in the dispersion of the main sequence around $\mathrm{M}_{r} \sim 12$. The final fits are listed in Table 4.2 and are shown in Figures 4.1, 4.3 and 4.4, along with other photometric parallax relations in the ugriz system.

\subsubsection{Comparison to Previous Relations}

As mentioned above, there are numerous photometric parallax relations published for the SDSS ugriz system. In Figures 4.5 - 4.7, the differences between the present relations and those found in Baraffe et al. (1998), West et al. (2005), Jurić et al. (2008), Sesar et al. (2008) and Golimowski et al. (2009) are plotted. These literature relations are drawn from a variety of sources: trig parallax stars (Golimowski et al., 2009), spectroscopic parallax (Hawley et al., 2002; West et al., 2005), statistical binarity and kinematic arguments (Jurić et al., 2008; Sesar et al., 2008, respectively) and model predictions (Baraffe et al., 1998). For the various CMRs (Figures 4.1, 4.3, 4.4), the rms scatter between the other relations and my own is consistent with the scatter seen in residuals $(\sigma \sim 0.40)$ from the stellar sample.

The best agreement among CMRs is between relations in this text and those of Golimowski et al. (2009). This is not surprising, since the relations are determined from the same underlying trig parallax sample. However, their relations are fit to a slightly different subsample of the PT observations and do not include the Dahn et al. (2002) and Vrba et al. (2004) stars. My relations are in broad agreement with 


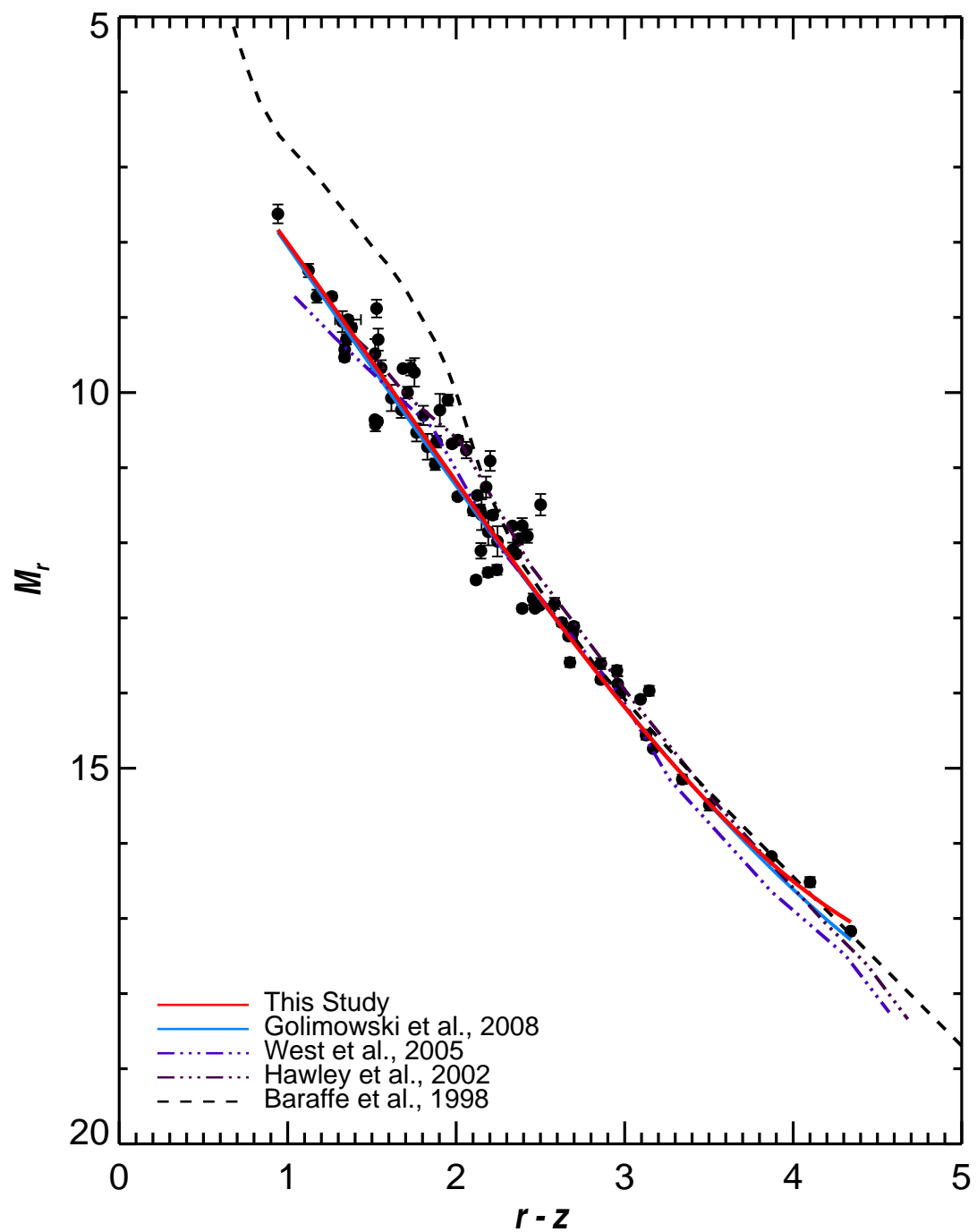

Figure $4.3 M_{r}$ vs. $r-z$ CMD. The parallax stars listed in Table 4.1 are shown as filled circles and the best fit line from Table 4.2 is the red solid line. The best fit from Golimowski et al. (2009) is shown as the blue line and the CMD from West et al. (2005) is plotted as the purple dash-dot line. The CMR of Hawley et al. (2002) is shown as the dark dash-dot line. The 5 Gyr isochrone from the Baraffe et al. (1998) models is shown as the dashed line. 


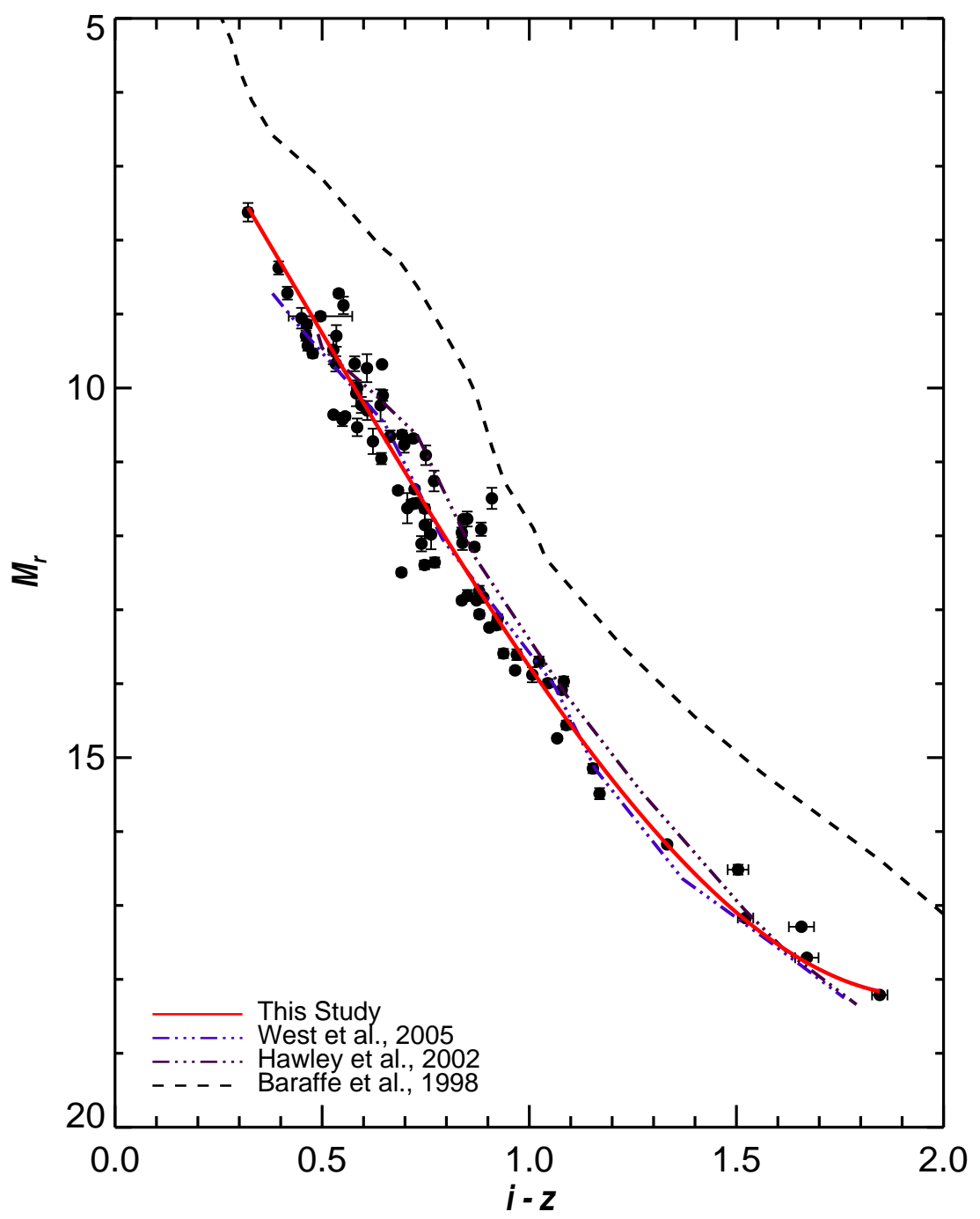

Figure $4.4 M_{r}$ vs. $i-z$ CMD. Same symbol definitions as Figure 4.3. Note the very poor agreement between the observations and model predictions. 
those reported by West et al. (2005). Their relation was based on a spectroscopic parallax calibration (originally described in (Hawley et al., 2002)) and their absolute magnitude calibrators were dominated by small numbers at early $(\sim \mathrm{M} 0)$ and late (M8-M9) spectral types, where the disagreement is the largest. Finally, for the blue end of the sample $(r-i<1.5)$ I can compare to the CMRs of Jurić et al. (2008) and Sesar et al. (2008). These photometric parallax relations are derived from statistical analyses that use kinematics (Jurić et al., 2008) or binarity (Sesar et al., 2008) to place strong constraints on the shape of the CMR. The absolute scaling is tied down by trig parallax stars (at red colors) and globular cluster isochrones (at the blue end). Despite the disparate techniques used to derive these CMRs, they agree quite well, with rms differences of $\sim 0.2$ mags.

For each CMD, the largest outlier is the model prediction of Baraffe et al. (1998). The differences observed between the present relations and those of the models cannot be attributed to the scatter in the main sequence. Instead, these differences are due to the fundamental limitations of current models of low-mass stars. Molecular bands, such as $\mathrm{TiO}, \mathrm{VO}$ and $\mathrm{H}_{2} \mathrm{O}$ dominate the SEDs of these stars. Unfortunately, the line lists for these molecules are incomplete and produce errors in the opacity calculation. Furthermore, at low temperatures $\left(T_{\text {eff }} \lesssim 2500 \mathrm{~K}\right)$, grain formation may influence the energy transport and is neglected by the Baraffe et al. (1998) models ${ }^{3}$. These shortcomings lead to large differences between the predicted and observed absolute magnitudes and colors of low-mass stars, and prohibit the models from being employed as useful indicators of absolute magnitude.

\subsubsection{Absolute Magnitude Transformations}

In order to transform a luminosity function (LF) measured in one filter, $\lambda$, into another filter, $\lambda^{\prime}$, one simply employs the following relation:

\footnotetext{
${ }^{3}$ Some models (Hauschildt \& Baron, 2005) now include the effects of dust grain formation, but do not predict ugriz photometry.
} 


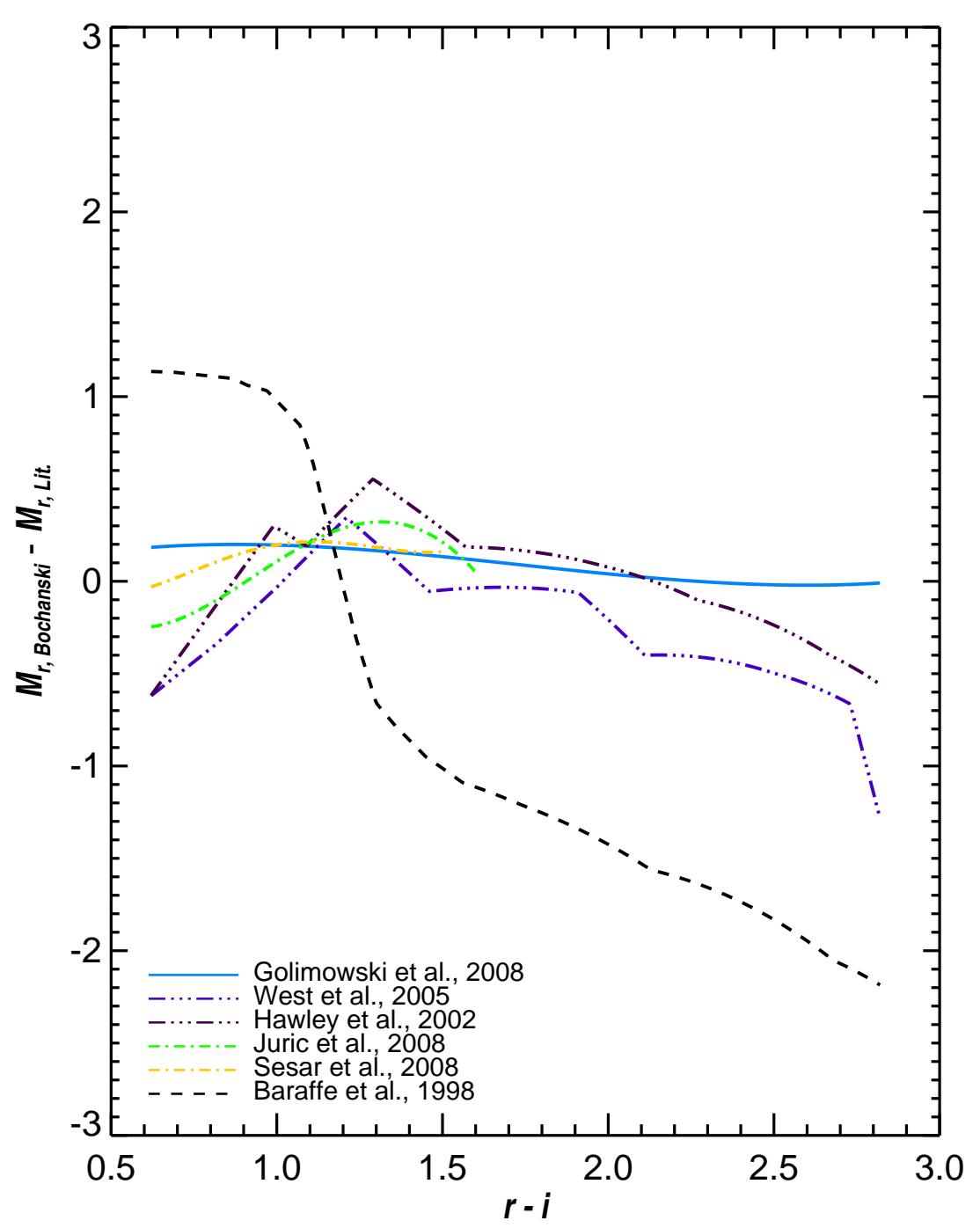

Figure 4.5 Differences between the $M_{r}$ vs. $r-i$ relations in Figure 4.1. The line styles are the same as Figure 4.1. Note that the majority of other photometric parallax relations agree at $\sim 0.2$ mags, while the 5 Gyr isochrone of Baraffe et al. (1998) fails to agree with the empirical results. 


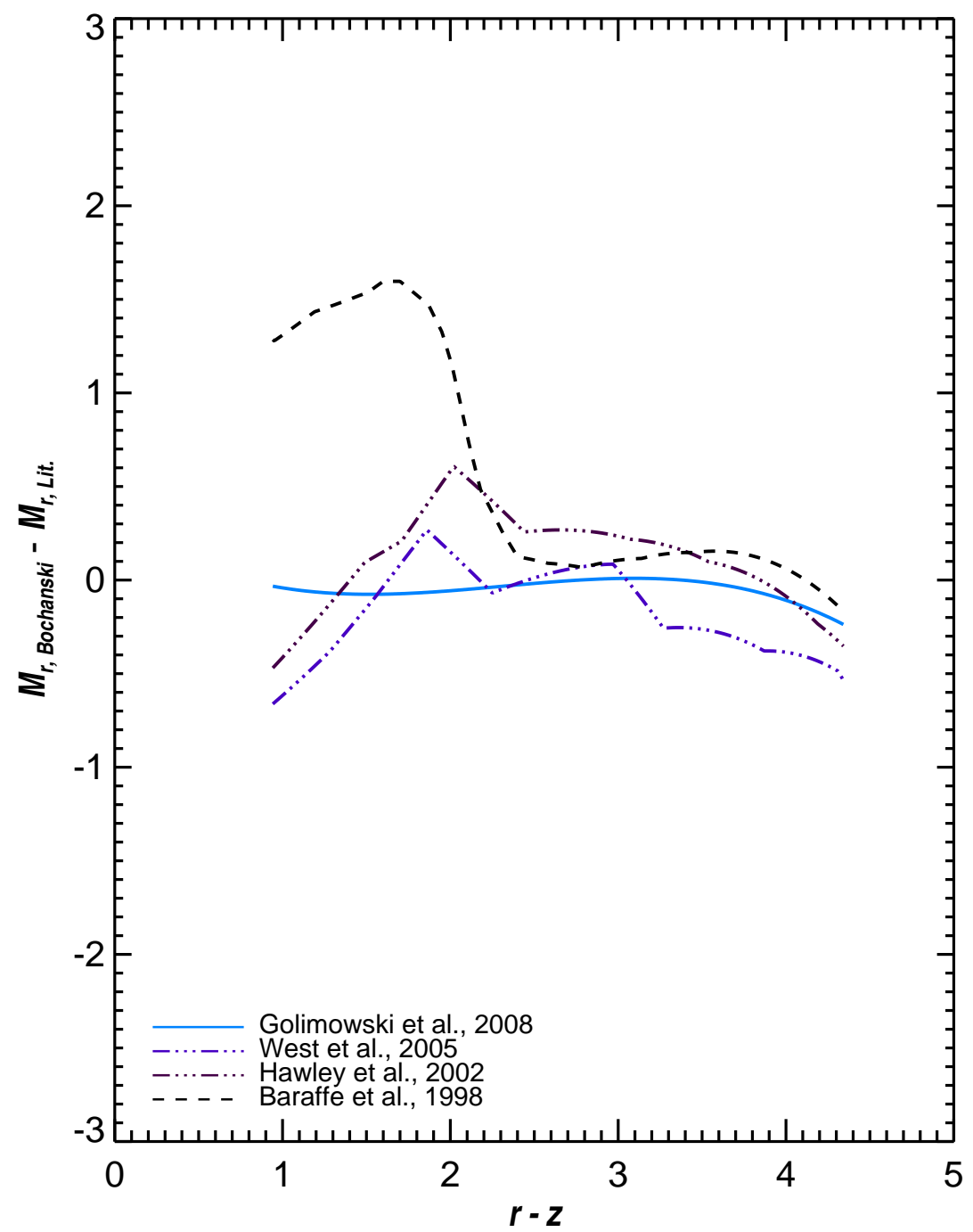

Figure 4.6 Differences between the $M_{r}$ vs. $r-z$ relations in Figure 4.3. Line styles are the same as Figure 4.3. As in Figure 4.5, there is excellent agreement between the Golimowski et al. (2009) CMR and our own. 


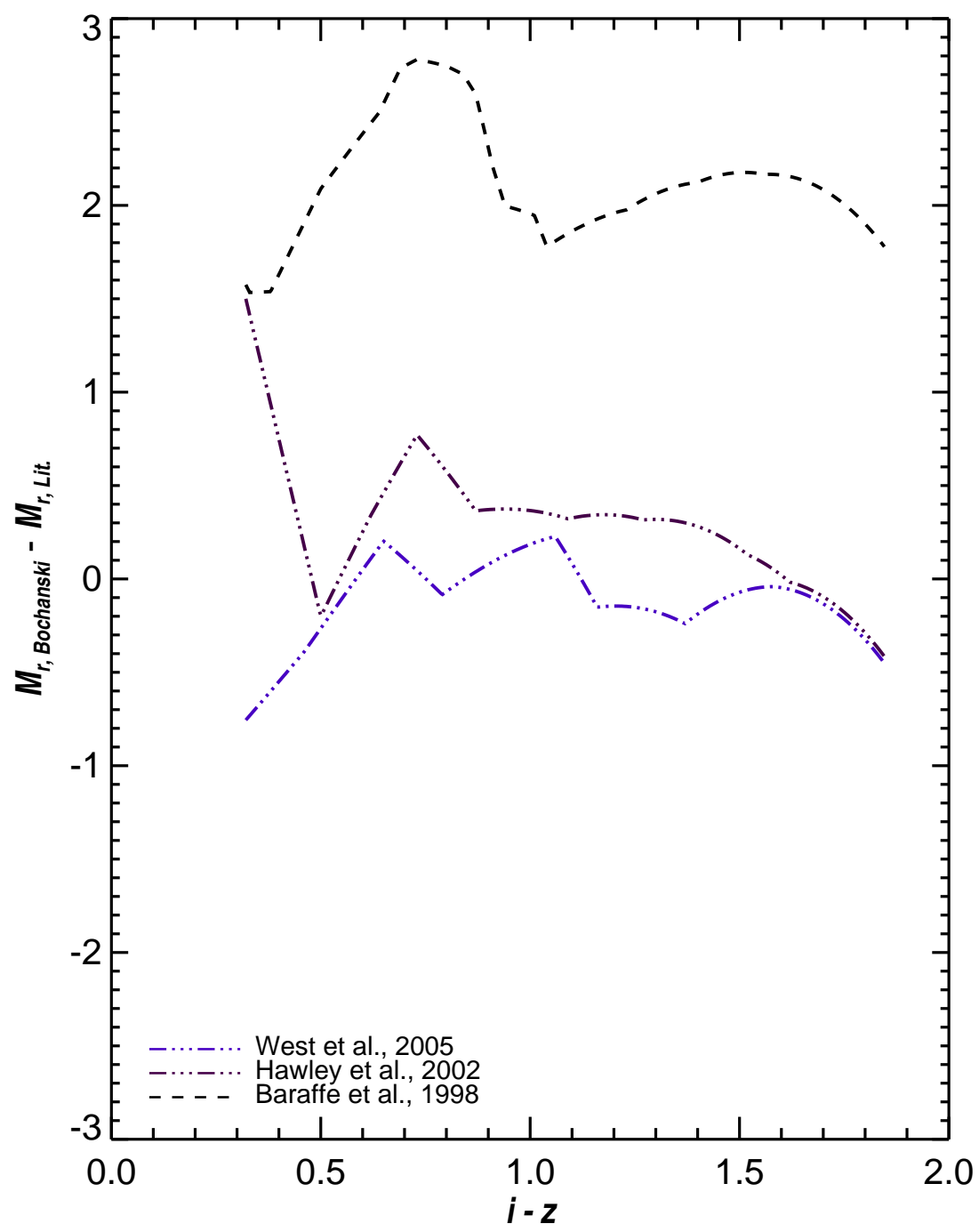

Figure 4.7 Differences between the $M_{r}$ vs. $i-z$ relations in Figure 4.4. Line styles are the same as Figure 4.4. 


$$
\Phi_{\lambda}^{\prime}=\frac{d N}{d M_{\lambda}} \frac{d M_{\lambda}}{d M_{\lambda}^{\prime}}=\Phi_{\lambda} \frac{d M_{\lambda}}{d M_{\lambda}^{\prime}}
$$

Using the nearby star sample, a simple fit between $M_{r}$ and $M_{J}$ was computed and used to transform the $M_{r}$ LF (see Chapter 5) into $M_{J}$. The transformed $M_{J}$ LF is readily compared to previous low-mass LFs measured in $J$ (e.g. Cruz et al., 2007; Covey et al., 2008, , see $§ 5.7 .2)$, and permits the application of empirical massluminosity relations (Delfosse et al., 2000) to derive a MF (see Chapter 6).

Using the photometry and parallaxes in Table 4.1 , a $5^{\text {th }}$ order polynomial was fit to $M_{J}\left(M_{r}\right)$. This fit is shown in Figure 4.8 and is given by:

$$
\begin{aligned}
M_{J} & =-60.49035+27.76258\left(M_{r}\right)-4.70659\left(M_{r}\right)^{2}+ \\
& +0.39887\left(M_{r}\right)^{3}-0.01650\left(M_{r}\right)^{4}+0.00027\left(M_{r}\right)^{5}
\end{aligned}
$$

and is valid for $7.6<M_{r}<17.7$. Taking the derivative of this equation and applying it to Equation 4.2 is straightforward. However, this method is not the only way to map $M_{r}$ into the $J$ band. By constructing a synthetic $r-J$ color from matched SDSS2MASS observations, the validity of Equation 4.3 can be tested. Using the relations from Table 2 of Covey et al. (2007b) and a $\left(M_{r}, g-i\right)$ relation measured from the nearby star sample, a synthetic $r-J$ color was constructed. Subtracting this $r-J$ color from $M_{r}$ yields $M_{J}$ and this relation is plotted as the blue dashed line in Figure 4.8. The two relations agree along most of the main sequence, then deviate by $\sim 1$ mag at $M_{r}>16$. This is not surprising, since this corresponds to $g-i \sim 4.1$, near the red limit of the Covey et al. (2007b) sample. Since the direct $M_{J}\left(M_{r}\right)$ relation in Equation 4.3 provides a better fit to the empirical measurements, it is adopted for the transformation between the $r$ and $J$ bands. 


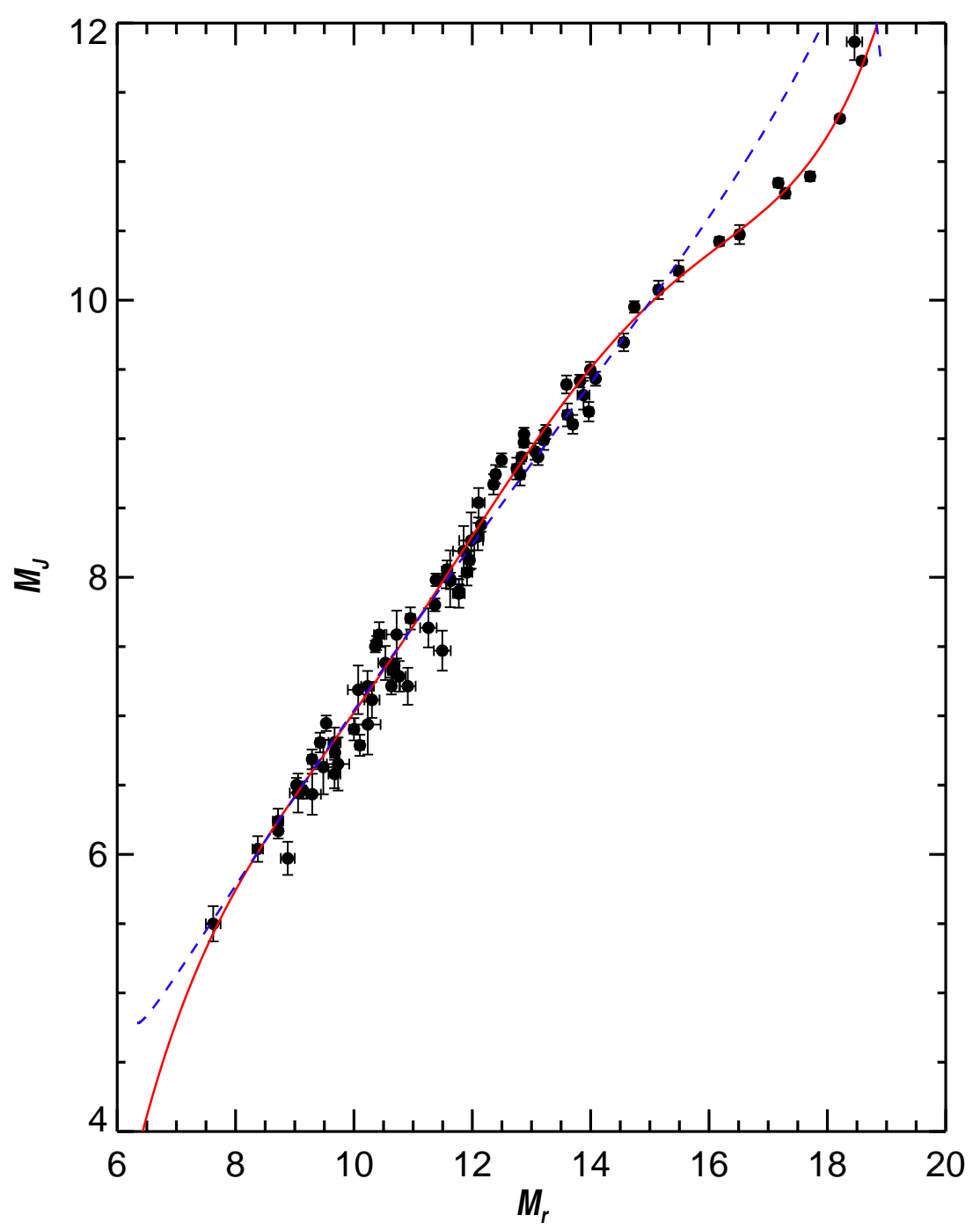

Figure $4.8 M_{J}$ magnitude vs. $M_{r}$ magnitude. Shown in red is a $5^{\text {th }}$ order best-fit polynomial, allowing the transformation from $r$ band to $J$ band absolute magnitudes. The dashed blue line is the relation constructed from a $\left(M_{r}, g-i\right)$ CMR and the analytic fits from Covey et al. (2007b). The rms scatter about the fit is $\sim 0.16$. 


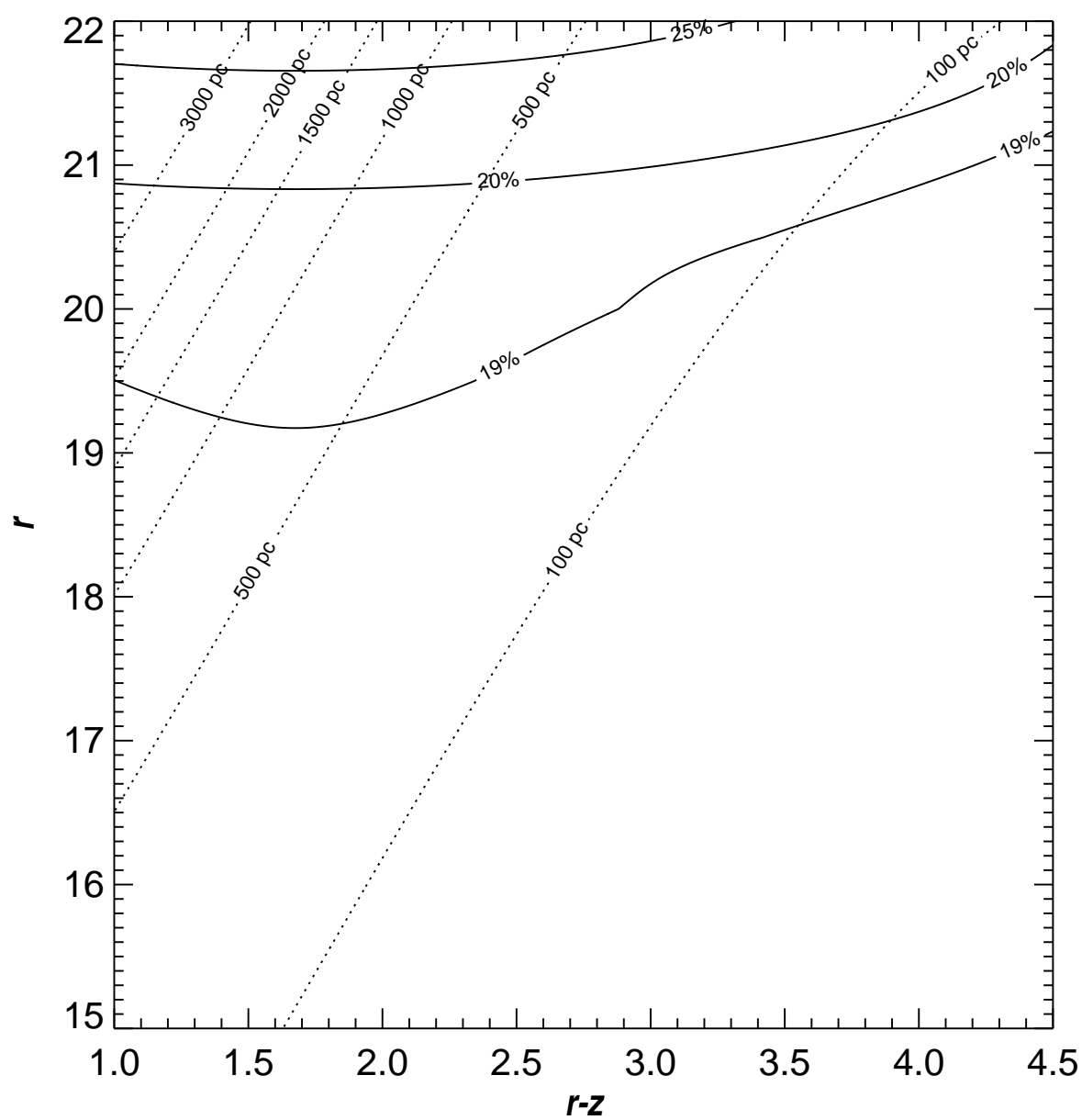

Figure 4.9 Fractional distance errors $\left(\sigma_{d} / d\right)$ as a function of $r-z$ color and $r$ magnitude. Notice for most of the sample, our distance errors are better than $20 \%$. This is a direct result of the accurate photometry from SDSS and the moderate slope of our adopted $\left(M_{r}, r-z\right)$ relation. Overplotted are lines of constant distance. 


\subsection{Conclusions}

Directly measuring trigonometric parallax distances for the millions of low-mass stars in SDSS is not currently possible. In order to estimate the distance to a given star, photometric parallax relations must be employed. Existing ugriz CMRs are calibrated from a variety of absolute magnitude standards and models fail catastrophically to reproduce the empirical data. Unfortunately, there is considerable scatter in the existing empirical relations for low-mass stars. Thus, in this chapter, a new set of CMR relations were computed.

This analysis incorporated new ugrizJHK photometry for 268 stars with known trigonometric parallaxes, kindly provided by Golimowski et al. (2009). After cleaning the sample of contaminants, such as white dwarfs, low-metallicity subdwarfs and binary stars, 86 stars with parallaxes known to better than $10 \%$ were used to determine the photometric parallax relations. The typical scatter in the relations is $\sigma_{M} \sim 0.4$ mag. In Figure 4.9, the fractional distance error as a function of apparent brightness $(r)$ and $r-z$ color is plotted, assuming the CMR from Table 4.2. Since most of the stars in the photometric sample (see Chapter 5) have distance $<2000$ pc, the expected fractional errors are $<20 \%$. While this is not as accurate as trignometric parallaxes, the CMRs presented here provide the best distance estimators for low-mass stars in SDSS. In the future, astrometric missions such as GAIA (Perryman et al., 2001) will greatly remedy this situation, providing accurate $\left(\sigma_{\pi} / \pi \sim 1 \%\right)$ parallaxes of stars to $V=20 \mathrm{mag}$.

The concept of absolute-magnitude transformations was also introduced in this chapter. These are simple polynomial fits to absolute-magnitude loci. Their utility will be evident in Chapter 5, as they permit the transformation of the LF to other filters. Thus, for a sample of stars with ugriz photometry, one could compute a $M_{J}$ LF. The typical caveats associated with transforming a LF to an MF apply for these relations as well. Steep slopes or sharp discontinuities could artificially 
introduce features in the transformed LF, so one must be cautious when interpreting any observed structure. 


\section{Chapter 5}

\section{LUMINOSITY FUNCTION OF FIELD LOW-MASS DWARFS}

\subsection{Introduction}

The stellar luminosity function (LF) is a fundamental observational quantity, crucial to understanding star formation and Galactic structure. The LF, defined as the number of stars per cubic parsec as a function of absolute magnitude, $\Phi(M)=d N / d M$, was among the first classical astronomical investigations, but uncertainty still exists today. Initial studies of the LF grew out of Galactic structure surveys. By the 1850s, there was significant evidence of a spread in the intrinsic luminosities of nearby stars (see Reid \& Hawley, 2005, and references therein). Attempts to include this spread in luminosity into star-counts culminated in the fundamental equation of stellar statistics (von Seeliger, 1898):

$$
A(m)=\omega \int \Phi(M-a(r)) \rho(r) r^{2} d r
$$

where $A(m)$ is the observed number of stars in a given apparent magnitude bin, $m$, over a solid angle $\omega$. This is the sum of stars of a given absolute magnitude, $M$, along a line of sight, $r$, with a density law given by $\rho(r)$ (and an allowance for extinction, $a(r))$. This formalism was popularized by Trumpler \& Weaver (1953).

The first major classical LF investigations were pioneered by a series of Dutch astronomers, beginning with Kapteyn, and followed by van Rhijn, Bok, Kuiper and Luyten (e.g., Kapteyn, 1902, 1914; Kapteyn \& van Rhijn, 1920; Bok \& Macrae, 1941; Kuiper, 1942), with the latter focusing on nearby stars selected by proper motions 
(e.g., Kuiper, 1942; Luyten, 1968). Luyten's work dominated studies of the low-mass LF, promulgating the concept of "reduced proper motion" which employed proper motion as a proxy for absolute magnitude (Luyten, 1923, 1968). During the 1970s, particular attention was focused on the LF of the lower $\left(L<0.1 L_{\odot}\right)$ main sequence. Considerable evidence compiled by Zwicky (1937), Rubin \& Ford (1970), Bosma (1978) and Oort $(1932,1960)$ suggested the presence of "missing mass", primarily arising from differences between dynamical mass estimates and observed mass distributions. Weistrop (1972) obtained photometry of $\sim 13,000$ stars and derived an LF with low-mass stellar densities 5-10 times greater than those measured by Luyten. A series of papers followed, exploring the validity of this new result ${ }^{1}$. This discrepancy was resolved a few years later, when re-analysis by Weistrop (1976) and Faber et al. (1976) revealed systematic errors in Weistrop's original photometric calibration.

Perhaps following in the footsteps of their predecessors, modern inquires into the stellar LF have met with controversy. While not plagued by the vitriol of the 1970s, there existed systematic differences in LFs derived from two different methods: volume-complete surveys of stars with trigonometric parallaxes in the Solar Neighborhood (e.g., Reid et al., 1995a; Reid \& Gizis, 1997; Reid et al., 2002) and deep, pencil-beam surveys of distant stars, using CMRs (similar to those in Chapter 4) to determine distances (e.g., Reid, 1982; Stobie et al., 1989; Gould et al., 1996; Zheng et al., 2001). Nearby LFs, which are limited in sample size, but resolve binary systems into individual stars and have accurate distances, found an excess of low-mass dwarfs at $M_{V} \gtrsim 13$ (Reid \& Gizis, 1997). On the other hand, photometric LF determinations, consistently found a declining LF at fainter absolute magnitudes. As an additional complication to the photometric method, the gradients introduced by Galactic structure $(\rho(r)$ from Equation 5.1), must be taken into account. Two

\footnotetext{
${ }^{1}$ Luyten, in particular, doubted the new LF measurement, in a series of caustic papers titled: "The Messiahs of the Missing Mass", "More Bedtime Stories from the Lick Observatory", and "The Weistrop Watergate". The author wonders if such writing would find its way into today's journals.
} 
solutions were proposed to explain the discrepancy between the two LFs: unresolved binaries in the photometric samples (Kroupa et al., 1991) and inaccurate CMRs (Reid \& Gizis, 1997; Chabrier, 2003b). Since many of the stars in the photometric sample are distant $(d \gtrsim 100 \mathrm{pc}$ ), any low-luminosity companions would be unresolved and not counted in the final LF. Thus, there is a bias against these faint companions, and this can potentially explain the systematic differences seen between the two LFs. Applying the correct CMR, as explained in Chapter 4, is a critical step in determining the photometric LF. If the CMR employed does not fit the stellar locus, either by smoothing over structure in the CMD (Reid \& Gizis, 1997) or neglecting metallicity (and thus absolute magnitude) gradients within the Galaxy (Zheng et al., 2001; Chabrier, 2003b), then systematic bias would skew the resultant LF. Both of these effects influence the photometric determination of the LF (Chabrier, 2003b).

With the advent of large digital sky surveys, such as SDSS (York et al., 2000) and 2MASS (Skrutskie et al., 2006), photometric measurements of the LF have again become popular. Bridging the gap between the two methods described above, Reid \& Cruz (2002) combined Luyten's NLTT catalog of proper motions with 2MASS JHK photometry to select candidate nearby stars. Their main objective was to complete the census of M and L dwarfs within 20 pc of the Sun (Reid et al., 2004). Covey et al. (2008) employed photometry from SDSS, 2MASS and the GSC to measure the stellar LF over 30 square degrees. The Covey et al. (2008) sample is among the largest-todate, using $\sim 30,000$ stars to measure the LF. These studies relied on spectroscopic followup, either for additional distance estimates (Cruz \& Reid, 2002; Reid et al., 2003a) or to quantify contamination from quasars and giants (Covey et al., 2008). The present study is compared to existing LF studies in Table 5.1. This table is not complete, but includes modern (since 1995) and older seminal studies of the field LF.

In this chapter, I introduce a new measurement of the LF using a stellar sample nearly three orders of magnitude larger than any previous study. In $\S 5.2$, I discuss the photometric sample of low-mass dwarfs from the SDSS. The analysis follows in 
$\S 5.3$ and $\S 5.4$ where I discuss a new approach to measuring the LF with large surveys. The systematic errors and biases of this analysis are discussed in 5.5. My results are compared to previous results for nearby Galactic structure in $\S 5.6$ and the stellar LF in $§ 5.7$. Finally, my conclusions follow in $\S 5.8$.

\subsection{Observations}

\subsubsection{SDSS Photometry}

The Sloan Digital Sky Survey (SDSS; York et al., 2000; Stoughton et al., 2002) ranks among the most ambitious astronomical surveys ever undertaken. The SDSS employed a 2.5m telescope (Gunn et al., 2006) at Apache Point Observatory (APO) to conduct an optical multi-wavelength in the ugriz filters, (Fukugita et al., 1996; Ivezić et al., 2007) photometric survey. Centered on the Northern Galactic Cap, the imaging data spans $\sim 10,000$ square degrees and is $95 \%$ complete to $r \sim 22.2$ (Stoughton et al., 2002; Adelman-McCarthy et al., 2007b). When the Galactic Pole is not visible from APO, about 300 sq. deg was scanned along the zero-declination region known as "Stripe 82" to empirically quantify completeness and photometric precision (Ivezić et al., 2007). In the latest public data release (DR6, Adelman-McCarthy et al., 2007b), over 287 million unique photometric objects have been identified. The photometric precision of SDSS is unrivaled for a survey of this size, with typical errors $\lesssim$ 0.02 mag (Ivezić et al., 2007). The sky was imaged using a time-delayed integration technique. Great circles on the sky were scanned along six camera columns, each consisting of five $2048 \times 2048$ SITe/Tektronix CCDs with an exposure time of $\sim 54$ seconds (Gunn et al., 1998). A custom photometric pipeline (Photo; Lupton et al., 2001) was constructed to analyze each image and perform photometry. Calibration onto a standard star network (Smith et al., 2002) was accomplished using observations from the "Photometric Telescope" (PT; Hogg et al., 2001; Tucker et al., 2006). Further discussion of PT calibrations for low-mass stars can be found in Davenport 
Table 5.1. Modern Major Low-Mass Stellar Field LF Studies

\begin{tabular}{lllllll}
\hline \hline \multicolumn{1}{c}{ Authors } & \multicolumn{1}{c}{$N_{\text {Stars }}$} & $\Omega$ (sq. deg.) & Filter(s) & Depth & Method & Notes \\
\hline Stobie et al. (1989) & 178 & 18.88 & $V, I$ & $I<16$ & Phot. $\pi$ & $V I$ photometry \\
Henry et al. (1994) & 92 & $\delta>-25^{\circ}$ & $V$ & $d=5 \mathrm{pc}$ & Trig. $\pi$ & Spec., CSN3 photometry \\
Reid et al. (1995a) & 520 & $\delta>-30^{\circ}$ & $V$ & $d \sim 20 \mathrm{pc}$ & Trig. $\pi$ & Spec., CNS3 photometry \\
Reid \& Gizis (1997) & 151 & $\delta>-30^{\circ}$ & $V$ & $d=8 \mathrm{pc}$ & Trig. $\pi$ & Spec., CNS3 photometry \\
Martini \& Osmer (1998) & 4,005 & 0.83 & $V$ & $V \sim 23.5$ & Phot. $\pi$ & $U B V R I$ photometry \\
Zheng et al. (2001) & 1,413 & $\sim 0.4$ & $V$ & $I \sim 26.5$ & Phot. $\pi$ & HST photometry \\
Reid et al. (2002) & 558 & $\delta>-30^{\circ}$ & $V$ & $d \sim 20$ pc & Trig. $\pi$ & Spec., CNS3 photometry \\
Cruz et al. (2007) & 99 & 14,823 & $J$ & $J \sim 17, d \sim 20$ pc & Phot. $\pi$, Trig. $\pi$ Spec., 2MASS photometry \\
Covey et al. (2008) & $\sim 29 \times 10^{3}$ & 29 & $J$ & $J=16.2$ & Phot. $\pi$ & SDSS \& 2MASS photometry \\
This Study & $\sim 15 \times 10^{6}$ & 8,417 & $r, J$ & $r=22$ & Phot. $\pi$ & SDSS photometry \\
\hline
\end{tabular}

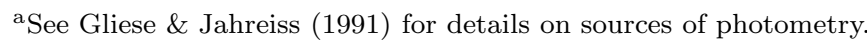

${ }^{\text {b }}$ The 8 pc sample was further extended by Reid et al. (1999) and Reid et al. (2003b) and presented in $J$ by Cruz et al. (2007).

${ }^{\mathrm{c}}$ Some of the HST observations in this study were presented by Gould et al. (1996) and Gould et al. (1997). 
et al. (2007). Absolute astrometric accuracy is better than 0.1" (Pier et al., 2003).

SDSS photometry has produced a throng of studies on the nature of low-mass dwarfs and Galactic structure. The average colors of $M$ dwarfs have been examined as a function of spectral type (Hawley et al., 2002; West et al., 2004, 2005; Bochanski et al., 2007b; Covey et al., 2007b) and metallicity (West et al., 2004). SDSS observations of these stars have also been used as tracers of local Galactic kinematics (Bochanski et al., 2007a; West et al., 2008) and Galactic structure (Chen et al., 2001; Jurić et al., 2008). This dissertation combines many of these efforts, simultaneously measuring Galactic structure and the luminosity and mass functions of low-mass stars.

SDSS-I concluded during 2005 and three projects now share time on the $2.5 \mathrm{~m}$ telescope as part of SDSS-II. The Legacy program is dedicated to finishing the imaging and spectroscopic observations of the original SDSS-I footprint. The SDSS Supernova Survey scans Stripe 82, searching for Type Ia supernovae candidates (Frieman et al., 2008). SEGUE is focused on Galactic studies, imaging lower Galactic latitudes and using modified spectroscopic targeting algorithms. Data from Legacy, SEGUE and the SDSS Supernova Survey are included in DR6. SDSS-II will conclude observing in the summer of 2008, and new projects for the $2.5 \mathrm{~m}$ telescope are currently being explored.

\subsubsection{Sample Selection}

I queried the SDSS catalog archive server (CAS) through the casjobs website ${ }^{2}$ for point sources with the following criteria:

- The point sources are found in the DR6 - Legacy footprint. The equatorial and Galactic maps of the sample are shown in Figure 5.1.

\footnotetext{
${ }^{2}$ http://casjobs.sdss.org/CasJobs/
} 
- The photometric objects were flagged as PRIMARY. This flag serves two purposes. First, it implies that the GOOD flag has been set, where GOOD = !BRIGHT \&\& (!BLENDED || NODEBLEND \| N_CHILD = 0). Second, it ensures that objects imaged multiple times are only counted once.

- The object was classified morphologically as a star. (TYPE $=6$ ).

- The photometric objects fell within the following brightness and color limits:

$$
\begin{gathered}
i<22.0, z<21.2 \\
r-i \geq 0.3, i-z \geq 0.2
\end{gathered}
$$

The first two cuts extend past the $95 \%$ completeness limits of the survey (Stoughton et al., 2002), but more conservative completeness cuts are enforced below. The latter two cuts ensure that the stars have red colors typical of M dwarfs (Bochanski et al., 2007b; Covey et al., 2007b).

This query produced 32,816,619 matches. Photometry must be complete and accurate to properly measure nearby Galactic structure and LF. Thus, the following additional criteria were imposed. To ensure completeness, I required $16<r<22$. These cuts conservatively account for the bright end of SDSS photometry, since the detectors saturate near 15th magnitude (Stoughton et al., 2002). At the faint end, the $r<22$ limit is slightly brighter than the formal $95 \%$ completness limits. 23,323,453 stars remain after these brightness cuts.

SDSS provides many photometric quality flags that quantify the reliability of each measured object. These flags are described in detail by Stoughton et al. (2002) and in the SDSS web documentation ${ }^{3}$. With the following series of flag cuts, the $\sim 23$

\footnotetext{
${ }^{3}$ http://www.astro.princeton.edu/ ${ }^{\sim}$ rhl/flags.html and http://www.sdss.org/dr6/products/catalogs/flags_detail.html provide excellent documentation of flag properties.
} 
million photometric objects are cleaned to a complete, accurate sample. Since only the $r, i$ and $z$ filters are used in this analysis, all of the following flags are only applied to those filters.

Bright stars will saturate a CCD after exceeding the dynamic range of the chip. Recorded photons "spill over" into neighboring pixels, severely distorting the shape of the point spread function (PSF) and prohibiting accurate photometry of its core. Hence, saturated photometry is not trustworthy and should be removed from the sample. First, saturated photometry is removed by selecting against objects with the SATURATED flag set. As seen in Figure 5.2, this cut removes mostly objects brighter than $15^{\text {th }}$ magnitude. To further clean saturated objects from the photometry, NOTCHECKED was cut against in $r, i$ and $z$. This flag marks areas on the sky where Photo did not search for local peaks, such as the cores of saturated stars. Similarly, the PEAKCENTER flag indicates that Photo did not accurately measure a star's PSF. This flag is set when the center of a photometric object is identified by the peak pixel, and not a more sophisticated centroiding algorithm. PEAKCENTER is selected against in $r, i$ and $z$. As seen in Figure 5.2, both of these flags compose a small fraction of the total number of observations and are more common near the bright and faint ends of the photometry. Saturated objects, as well as very low signal-to-noise observations, will fail these tests.

The last set of flags examines the structure of the PSF after it has been measured. Cosmic Ray (CR) is set when the star's PSF contains a sharp spike that has been interpolated over. This does not mean that the object was interpreted to be a cosmic ray. According to Stoughton et al. (2002) this should not significantly affect the measured photometry, so this flag was ignored. This is significant, since the CR flag is set for over 800,000 stars in the sample (see Figure 5.2 and Table 5.2). The PSF_FLUX_INTERP flag is tripped when over 20\% of the star's PSF is interpolated. While Stoughton et al. (2002) claims that this procedure generally provides trustworthy photometry, it warns of cases where this may not be true. Visual inspection 
of the $(r-i, i-z)$ color-color diagram in Figure 5.3 confirms the latter, showing a wider locus than other flag cuts. The INTERP_CENTER flag is set when a pixel within three pixels of the center of a star is interpolated. The $(r-i, i-z)$ colorcolor diagram of objects with INTERP_CENTER set is also wide and the Gaussian fit to the PSF could be significantly affected by an interpolated pixel near its center (Stoughton et al., 2002). Thus, stars with these flags set are removed. Finally, BAD_COUNTS_ERROR is set when a significant fraction of the star's PSF is interpolated over and the photometric error estimates should not be trusted. Table 5.2 lists the number of stars in the sample with each flag set. For the final "clean" sample, I define the following metaflag:

$$
\begin{gathered}
\text { clean }=\left(\text { !SATURATED }_{r, i, z} \text { AND } \text { !PEAKCENTER }_{r, i, z}{\text { AND } \text { INOTCHECKED }_{r, i, z}}\right. \\
\text { AND } ! \text { PSF_FLUX_INTERP }{ }_{r, i, z} \text { AND !INTERP_CENTER }{ }_{r, i, z} \text { AND } \\
\text { !BAD_COUNTS_ERROR } \left.{ }_{r, i, z} \text { AND }(16<\text { psfmag_r }<22)\right)
\end{gathered}
$$

The stellar sample after flag cuts is composed of 21,418,445 stars.

The final cut applied to the stellar sample is based on distance. As explained below in $\S 5.3 .2$, stellar densities are calculated within a $4 \times 4 \times 4 \mathrm{kpc}^{3}$ cube centered on the Sun. Thus, only stars within this volume are retained, and the final number of stars in the sample is $15,340,771^{4}$.

The final distributions in $r, i$, and $z$ are shown in Figure 5.4. Note that in each filter, there is an increase in star-counts up to the completeness limits of SDSS $(r<22$, $i<21.3, z<20.5$ ), with a rapid falloff thereafter. In Figure 5.5, histograms of the $r-i, i-z$ and $r-z$ colors are shown. These color histograms map directly to absolute magnitude, since CMRs are used to estimate absolute magnitude and distance. The structure seen in the color histograms at $r-i \sim 1.5, i-z \sim 0.7$ and $r-z \sim 2.2$ result from the convolution of the peak of the LF with the Galactic stellar density profile

\footnotetext{
${ }^{4}$ The reported number is based on the $\left(M_{r}, r-z\right)$ CMR. As explained below, changes to the CMR can add or subtract stars from the volume.
} 

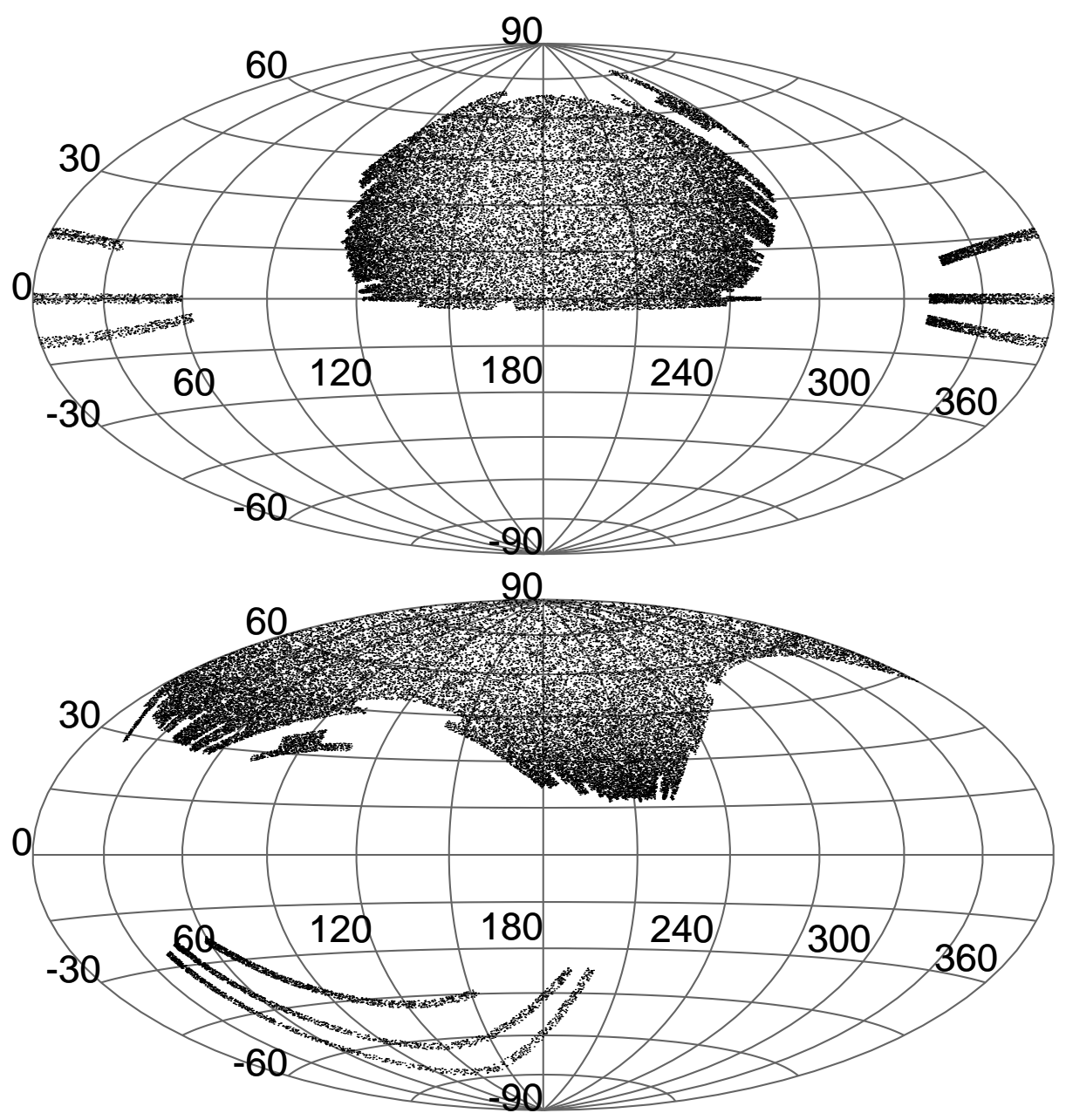

Figure 5.1 The Aitoff projections of the SDSS DR6 Legacy footprint in equatorial (top panel) and Galactic (bottom panel) coordinates. To aid figure clarity, only $0.2 \%$ of the final sample is shown. 

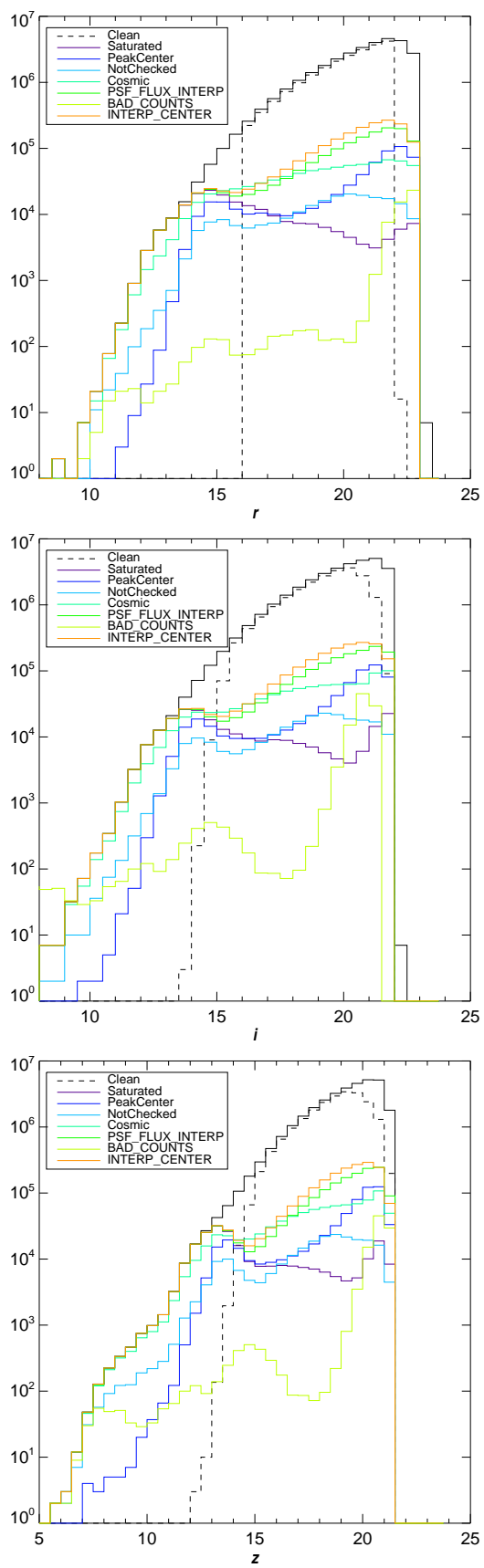

Figure 5.2 Histograms in $r, i$ and $z$ showing the effects of flag cuts on the sample. Each flag is labeled with a different color as noted in the legend. The "clean" sample (dashed line) is complete from $16<r<22$. 

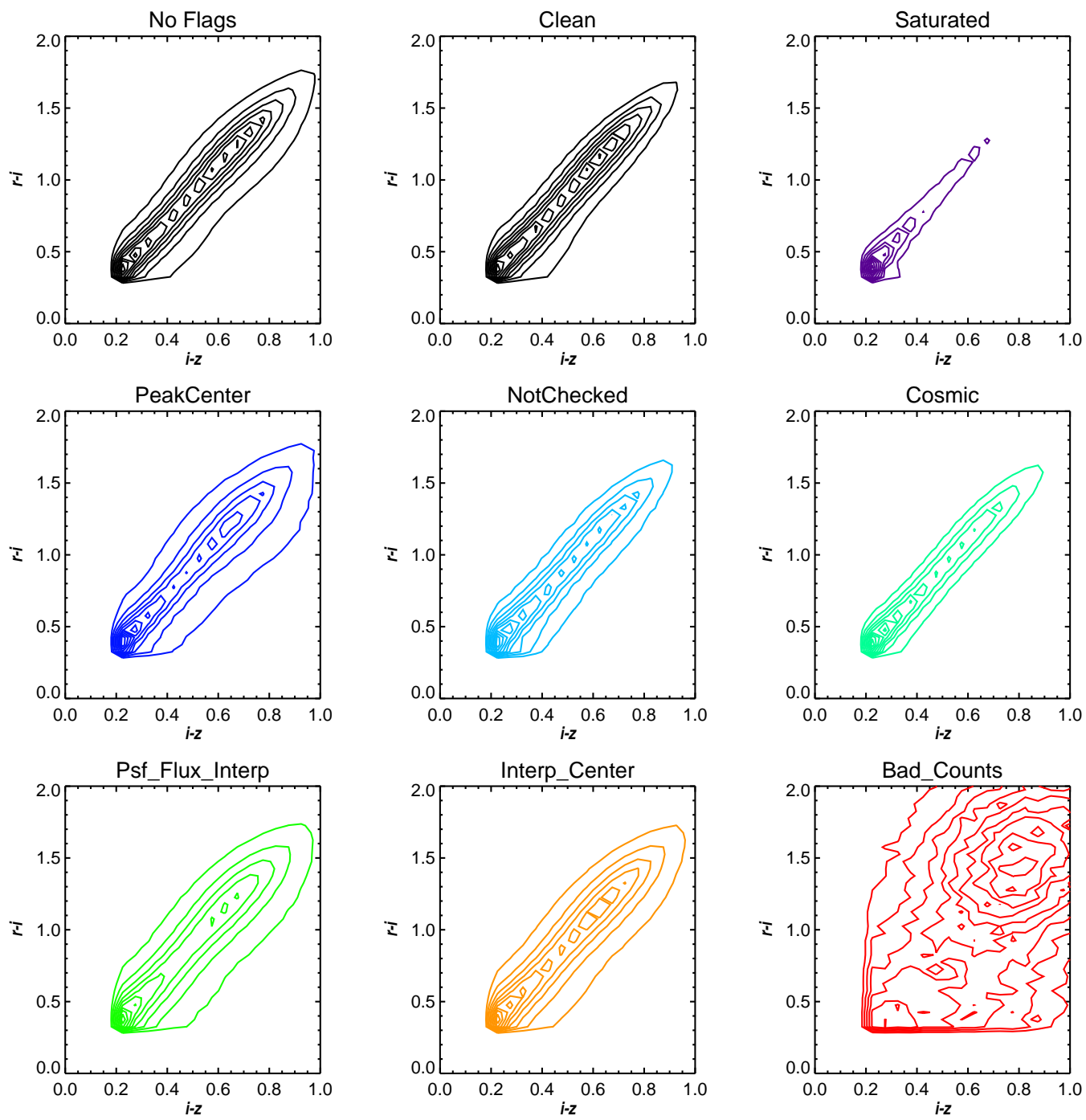

Figure 5.3 Shown in the panels are the $r-i, i-z$ color-color diagrams for the various flag cuts discussed above. The contours increase at $10 \%$ intervals. Of note are the relatively wide loci of the objects with the PSF_FLUX_INTERP and INTERP_CENTER flags set. BAD_COUNTS_ERROR objects also demonstrate considerable scatter. SATURATED objects are mostly bluer, indicating that they are probably higher luminosity. 
Table 5.2. Flag Cuts in SDSS sample

\begin{tabular}{lll}
\hline \hline \multicolumn{1}{c}{ Flag } & Num. of Stars & \multicolumn{1}{c}{ Description } \\
\hline SATURATED & 246,316 & Pixel(s) saturated within the PSF \\
PSF_FLUX_INTERP & $1,609,439$ & > 20\% of the PSF interpolated \\
INTERP_CENTER & $1,993,063$ & Interpolated pixel within 3 pixels of the center \\
BAD_COUNTS_ERROR & 97,697 & Significant interpolation, underestimated errors \\
PEAKCENTER & 598,108 & Center found by peak pixel, centroiding failed \\
NOTCHECKED & 230,375 & Peak of PSF not examined, probably saturated \\
COSMIC & 861,206 & CR hit within PSF, should not affect photometry \\
"CLEAN" & $21,418,445$ passed & Stars that passed quality \& completeness cuts \\
"CUBE" & $15,340,771$ & "Clean" stars within 4 kpc ${ }^{3}$ cube \\
\hline
\end{tabular}

over the volume probed by SDSS. Removing the density gradients and normalizing by the volume sampled constitutes the majority of the effort needed to convert these color histograms into a LF. The $(g-r, r-i)$ and $(r-i, i-z)$ color-color diagrams are shown in Figure 5.6, along with the model predictions of Baraffe et al. (1998) and Girardi et al. (2004). It is clearly evident that the models fail to reproduce the stellar locus, with discrepancies as large as $\sim 1$ mag. This further supports the argument in $\S 4.4$, that these models are imperfect and should not be used to form CMRs.

There are two additional sources of uncertainty concerning the ugriz colors of low-mass stars. As noted in Covey et al. (2008), spectral synthesis of L and T dwarfs have revealed differing responses in the $z$ band, inducing errors up to 0.1 mags (Burgasser, private communication). Furthermore, Ivezić et al. (2007) discuss small $(\sim 1 \%)$ differences in the response curves of the CCDs in each camera column. As all of the stars in this sample are M dwarfs, I have not explicitly corrected for the former effect, and neglect the latter, since it a small $(\lesssim 1 \%)$ effect. 

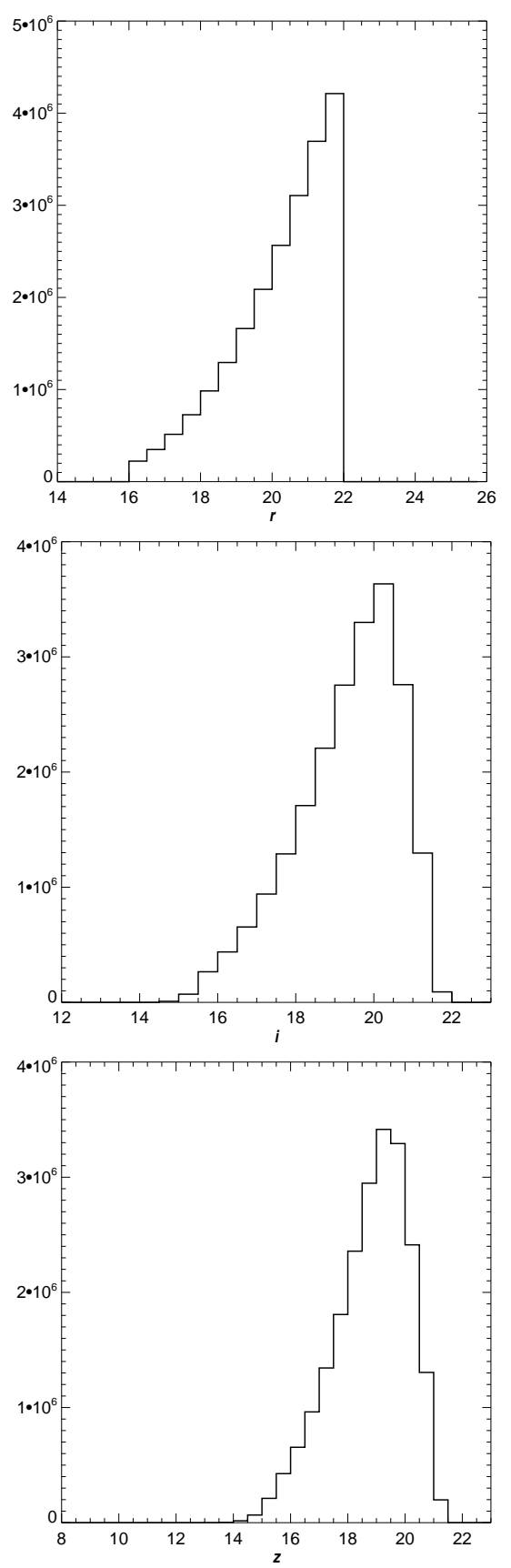

Figure 5.4 Histograms of brightness for the final stellar sample in $r, i$, and $z$. 

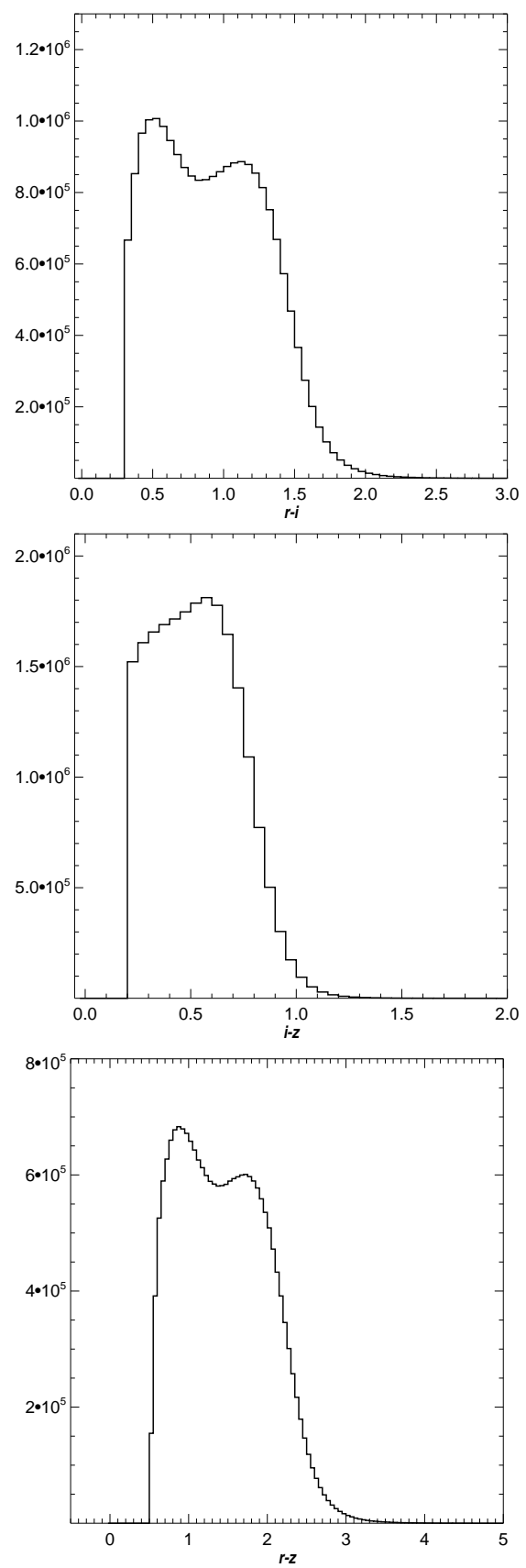

Figure 5.5 Histograms of color for the final stellar sample in $r-i, i-z$, and $r-z$. 

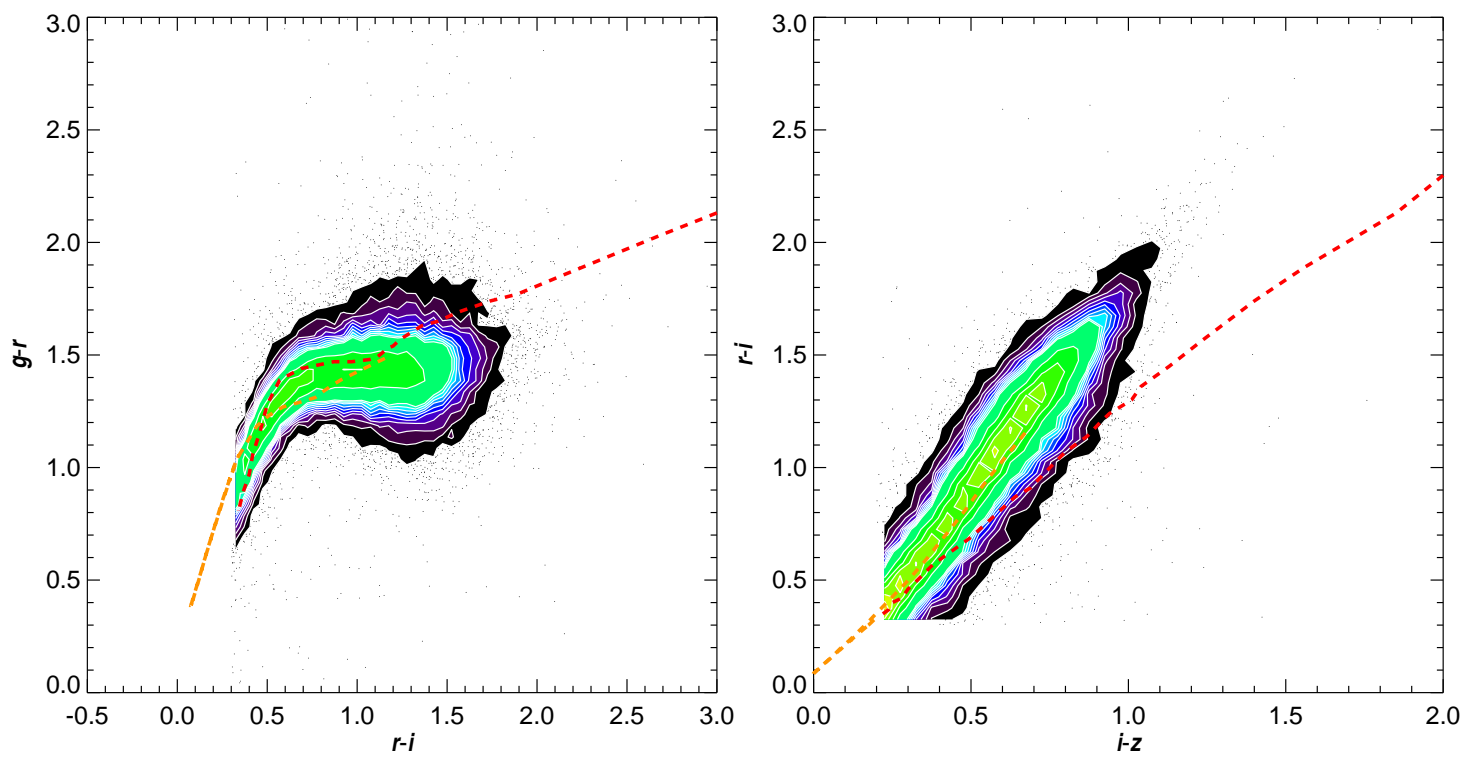

Figure 5.6 Color-color diagrams of the final photometric sample with the 5 Gyr isochrones of (Baraffe et al., 1998) (red dashed line) and (Girardi et al., 2004) (yellow dashed line) overplotted. The contours represent $0.2 \%$ of our entire sample, with contours increasing every 10 stars per 0.05 color-color bin. Note that the model predictions fail by more than a magnitude in some locations of the stellar locus. 


\subsection{Analysis: Stellar Density Maps}

In the following three sections, I motivate and explain a new method for measuring the LF from large areal surveys, solving simultaneously for Galactic structure and the luminosity function. This approach is compared to existing techniques, particularly the studies of Covey et al. (2008) and Jurić et al. (2008), and I comment on its application to future survey observations.

\subsubsection{Background}

Traditionally, investigations of the field LF use two distinct methods: counting stars within a volume-complete sample (e.g., Dahn et al., 1986; Henry et al., 1994; Reid et al., 2002) or deep, magnitude-limited pencil beam surveys, with solid angles up to a few square degrees (e.g., Stobie et al., 1989; Gould et al., 1996; Zheng et al., 2001; Covey et al., 2008). Since the SDSS is a flux-limited survey, the present study falls into the latter category, as noted in Table 5.1.

As mentioned above, Covey et al. (2008) studied a sample of stars with SDSS, 2MASS and GSC photometry to measure the LF over 30 sq. degrees. Covey et al. (2008) also obtained extensive spectroscopic followup, quantifying contamination and biases in SDSS and 2MASS photometry. Their work serves as a "Calibration Region" for my thesis investigation. In particular, I extend the initial study of Covey et al. (2008) in two specific categories. First, the present study is solely comprised of SDSS photometry, extending the faint limit of the survey by nearly 3 magnitudes (see Figure 3 of Covey et al., 2008). This increases the sample size and photometric precision, as SDSS photometry has errors of $<0.05$ mags, while the highest quality 2MASS data $($ ph_qual = "A") have average errors nearly twice as large. Finally, my study samples 8,400 sq. degrees, nearly 300 times the area surveyed by Covey et al. (2008). This large sky coverage represents the main challenge in measuring the LF of this sample. Most of the previous studies in Table 5.1 either assumed a uniform density distribution 
(for nearby stars) or calculated a Galactic density profile, $\rho(r)$ along one line of sight. Yet, none of these samples approached the size of the current investigation. With millions of stars spread over nearly $1 / 4$ of the sky, numerically integrating Galactic density profiles for each star is computationally prohibitive.

To solve this problem, I introduce a new technique for measuring the luminosity function. First, absolute magnitudes are assigned and distances to each star are computed using the CMRs from Chapter 4. Next, a small range in absolute magnitude (0.5 mag) is selected and the stellar density is measured in situ as a function of Galactic radius $(R)$ and Galactic height $(Z)$. This range in absolute magnitude was selected to provide high resolution of the LF, with an large number of stars in each bin. Finally, a Galactic profile is fit to the $R, Z$ density maps, solving for the shape of the thin and thick disks, as well as their local densities. The shape of the stellar density distribution is constrained to be the same for each absolute magnitude slice, as they should sample stars of similar mass and age. The luminosity function is then constructed by combining the local density of each absolute magnitude slice.

\subsubsection{Density Calculation}

To assemble a $(R, Z)$ density map, an accurate count of the number of stars in a given $R, Z$ bin, as well as the volume spanned by each bin is necessary. A cylindrical $(R, Z, \phi)$ coordinate system was taken as the natural coordinates of stellar density in the Milky Way. In this frame, the Sun's position is set at $R_{\odot}=8.5 \mathrm{kpc}$ (Kerr \& Lynden-Bell, 1986) and $Z_{\odot}=15$ pc above the Plane (Cohen, 1995; Ng et al., 1997; Binney et al., 1997; Jurić et al., 2008). Azimuthal symmetry is assumed and was tested explicitly by Jurić et al. (2008) and found to be appropriate for the local Galaxy. Furthermore, traditional stellar density profiles (Equations 5.8 - 5.10) do not incorporate azimuthal variations. Thus, the following analysis is carried out in $R$ and 
$Z$. I stress that this is not the $\phi=0$ plane $^{5}$. Rather, the density maps are averaged over $\phi$, collapsing the three-dimensional SDSS volume into a two-dimensional density map.

The coordinate transformation from a spherical coordinate system $(\ell, b$, and $d)$ to a cylindrical $(R, Z)$ system is performed with the following equations:

$$
\begin{gathered}
R=\sqrt{(d \cos b)^{2}+R_{\odot}\left(R_{\odot}-2 d \cos b \cos \ell\right)} \\
Z=Z_{\odot}+d \sin \left(b-\arctan \left(Z_{\odot} / R_{\odot}\right)\right)
\end{gathered}
$$

where $d$ is the distance (as determined by Equation 4.1 and the $\left(M_{r}, r-z\right) \mathrm{CMR}$ ), $\ell$ and $b$ are Galactic longitude and latitude, respectively, and $R_{\odot}$ and $Z_{\odot}$ are the position of the Sun, as explained above. An example of the star counts as a function of $R$ and $Z$ is shown in Figure 5.7.

To estimate the volume sampled by each $R, Z$ bin, the following numerical method was used. First, a suitable bin size was chosen for $R$ and $Z$. This width needed to be large enough to contain many stars (to minimize Poisson noise), but small enough to accurately resolve the structure of the thin and thick disks. The $R, Z$ bin size was set at $25 \mathrm{pc}$. Next, a $4 \times 4 \times 4 \mathrm{kpc}$ cube of "test" points was laid down, centered on the Sun, at uniform intervals $1 / 10$ th the $R, Z$ bin size (every $2.5 \mathrm{pc}$ ). This cube essentially discretizes the volume, with each point corresponding to a fraction of the total volume. Here, each grid point represents:

$$
k=\frac{\text { Volume }}{N_{\text {points }}}=\frac{\left(4 \times 10^{3} \mathrm{pc}\right)^{3}}{(4000 / 2.5)^{3} \text { points }}=15.625 \mathrm{pc}^{3} \text { point }^{-1}
$$

\footnotetext{
${ }^{5}$ In other words, this is not the plane that is normal to the Galactic Plane $(Z=0)$ and intersects the Sun and Galactic Center.
} 


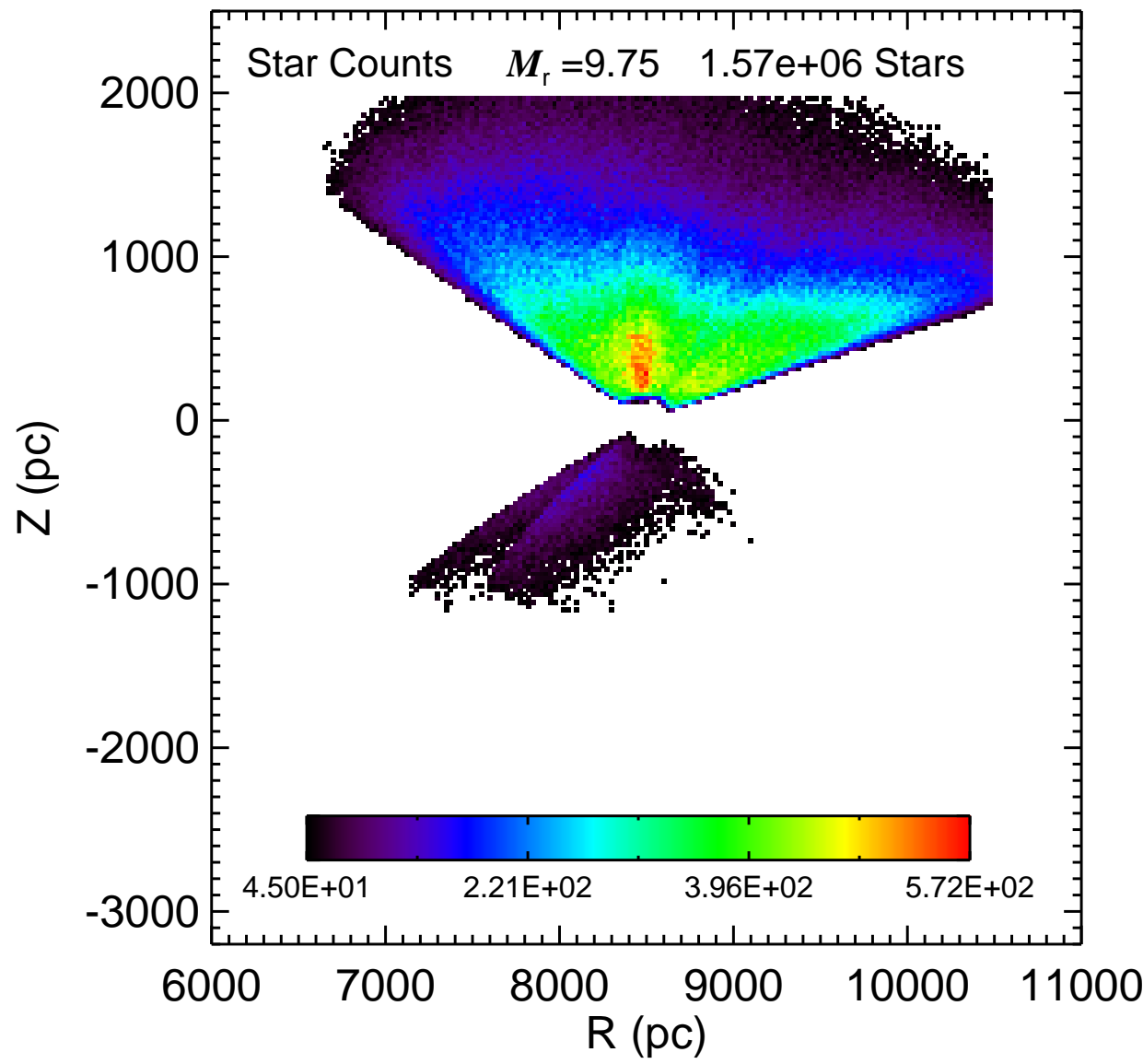

Figure 5.7 Star counts as a function of Galactic $R$ and $Z$ for a 0.5 magnitude slice in absolute magnitude centered on $M_{\mathrm{r}}=9.75$. The color bar on the lower part of the plot displays the scale of the image. The number of stars in this absolute magnitude slice is at the top of the plot. The majority of the stars in the sample are found in the northern Galactic hemisphere, since SDSS was centered on the Northern Galactic Cap. 
The volume of an arbitrary shape ${ }^{6}$ is straightforward to calculate: Simply count the points that fall within the shape, and multiply by $k$. This method is related to Monte Carlo integration ${ }^{7}$. The $\alpha, \delta$ and distance of each point was calculated and compared to the SDSS volume. If the test point fell within the SDSS footprint at a distance appropriate for the absolute magnitude slice and SDSS bright and faint limits $(16<r<22)$, it was recorded. The number of test points in each $R, Z$ bin was summed and multiplied by $k$ to get the final volume corresponding to that $R, Z$ bin. This process was repeated for each absolute magnitude slice. Since the volumes are fully discretized, the error associated with $N_{\text {points }}$ is Poisson distributed (and straightforward to calculate). A fiducial example of the volume calculations is shown in Figure 5.8.

After calculating the volume of each $R, Z$ bin, the density (in units of stars $\mathrm{pc}^{-3}$ ) is simply:

$$
\rho(R, Z)=\frac{N(R, Z)}{V(R, Z)}
$$

with the error given by:

$$
\sigma_{\rho}=\rho \sqrt{\left(\frac{\sqrt{N(R, Z)}}{N(R, Z)}\right)^{2}+\left(\frac{k \sqrt{N_{\text {points }}(R, Z)}}{V(R, Z)}\right)^{2}}
$$

Fiducial density and error maps are shown in Figures 5.9 and 5.10.

\subsubsection{Disk Density Profiles}

To accurately measure the local density of each absolute magnitude slice, the shape of the Disk must be accounted for. Determining the stellar density distribution of the Milky Way is a classical astronomical question. Indeed, $\rho(r)$ in Equation 5.1

\footnotetext{
${ }^{6}$ Say, for example, the SDSS DR6 footprint.

${ }^{7} \mathrm{~A}$ classic example of Monte Carlo integration is the estimation of $\pi$ by measuring the area of a circle with diameter $d$ inside a square of the same length.
} 


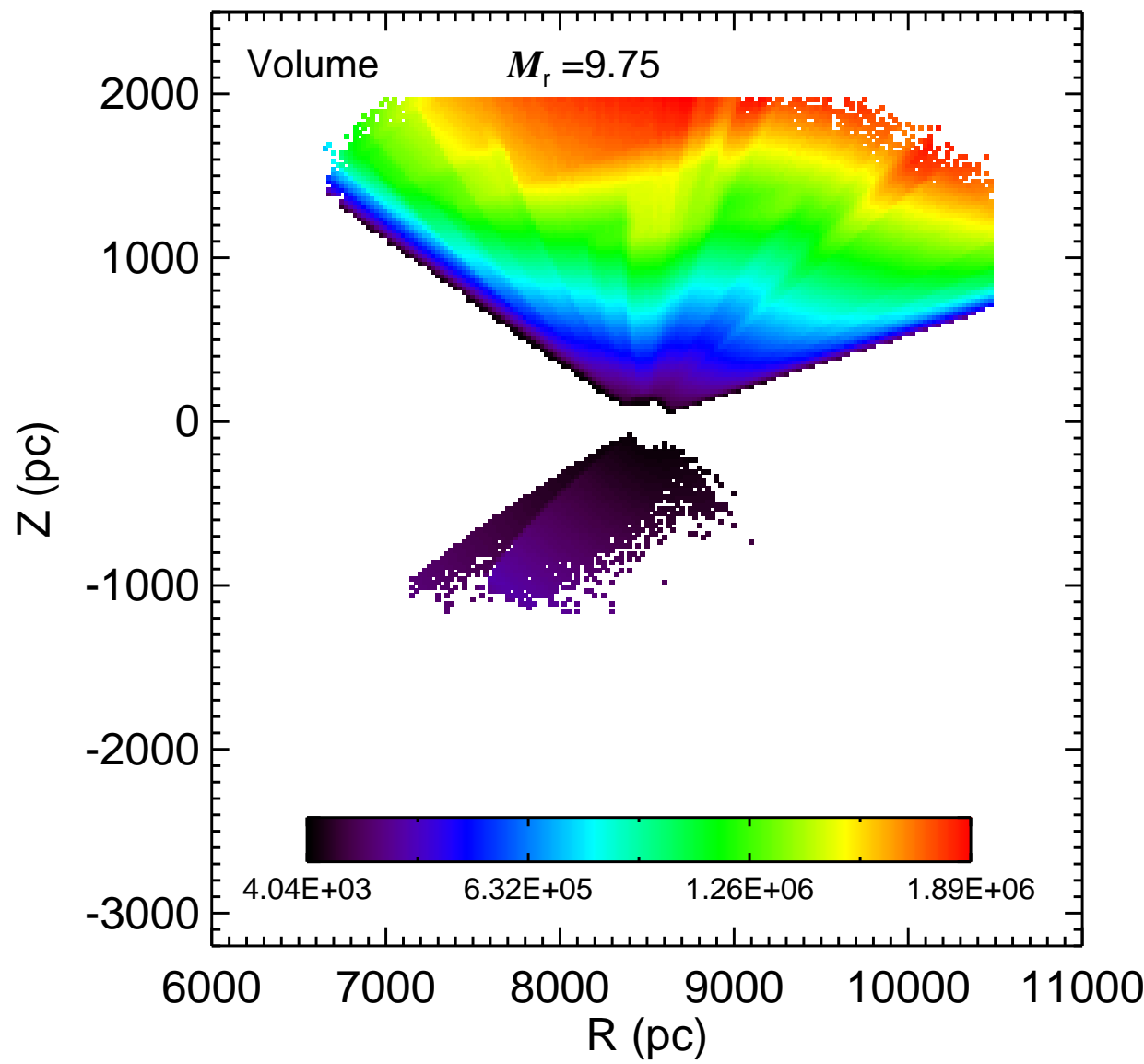

Figure 5.8 Volume probed by SDSS imaging as a function of Galactic $R$ and $Z$ for one $0.5 \mathrm{mag}$ slice at $M_{\mathrm{r}}=9.75$. The corresponding scale (in $\mathrm{pc}^{3}$ ) is at the bottom of the plot. 


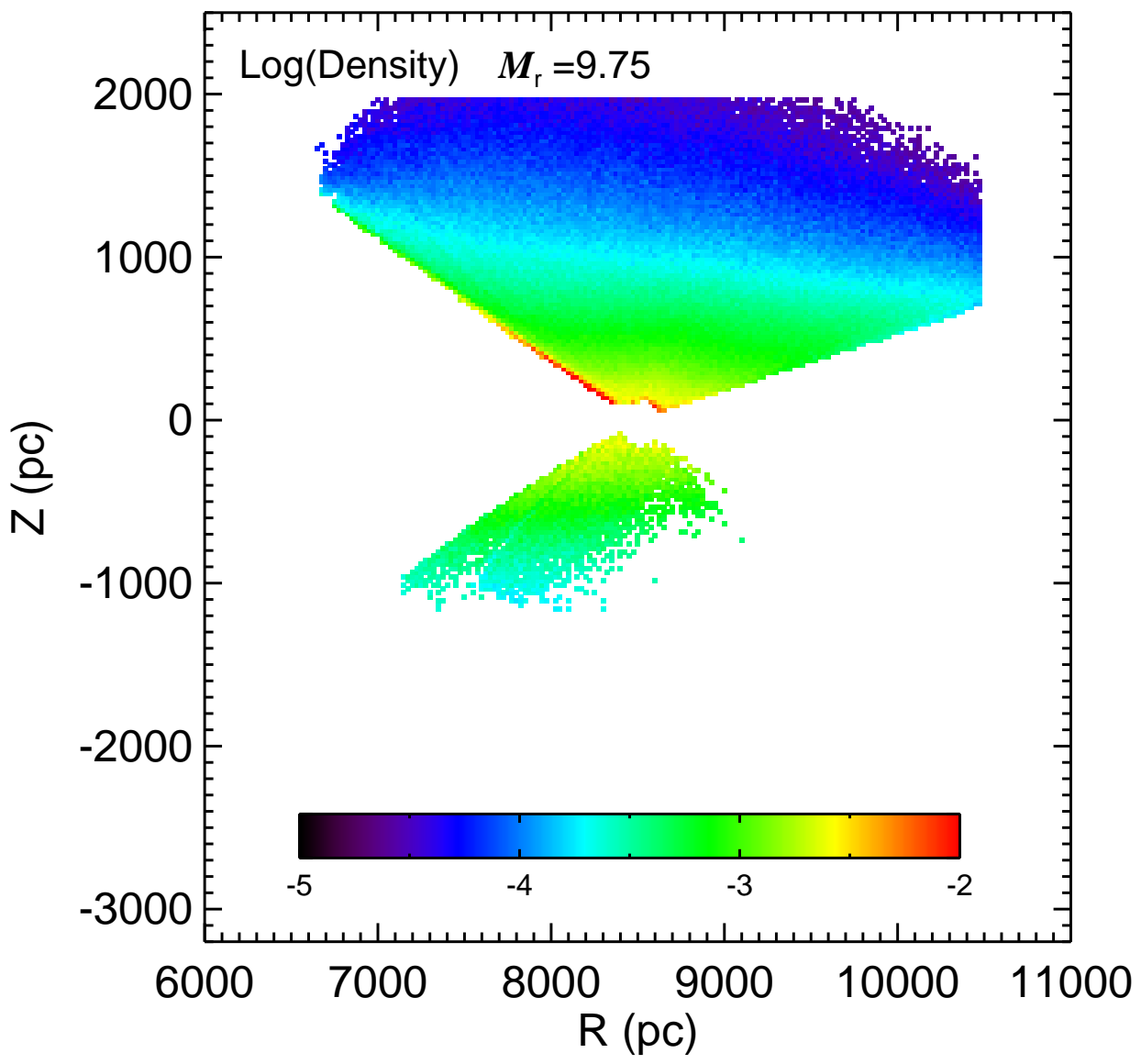

Figure 5.9 Density (in stars $\mathrm{pc}^{-3}$ ) as a function of Galactic $R$ and $Z$. The logarithmic scale is shown beneath the density map. The disk structure of the Milky Way is clearly evident, with a smooth decline towards larger $R$, and an increase in density approaching the Plane $(Z=0)$. 


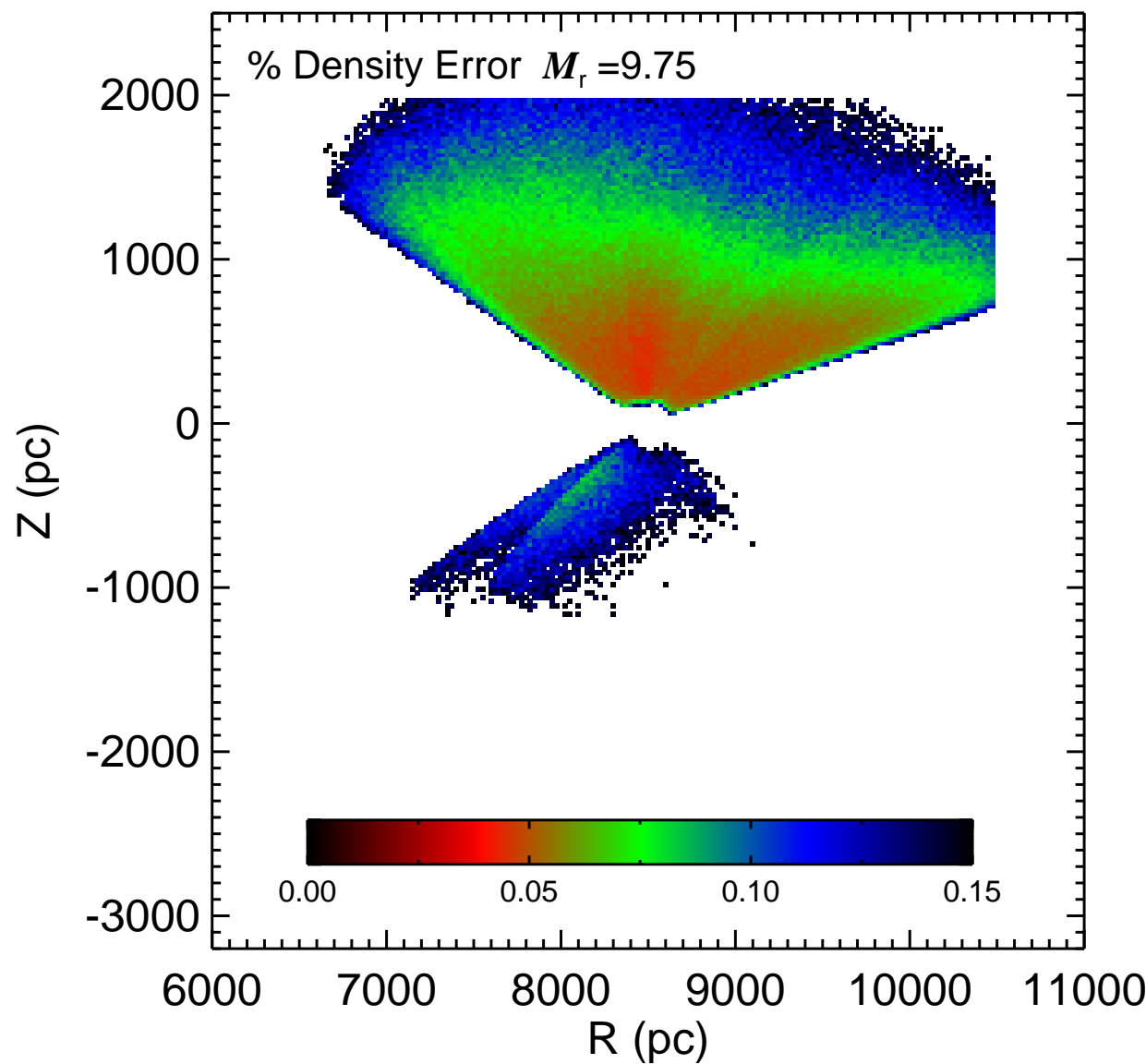

Figure 5.10 The fractional error in density as a function of $R$ and $Z$. As in the previous figures, the scale is below the map. The errors, calculated in Equation 5.6 are $\lesssim 7 \%$ for the majority of the sample. 
carries the same mathematical power as $\Phi(M)$. For a detailed description of historical analyses of the Milky Way's stellar profile, the reader is referred to Bok (1937). Modern determinations of the radial and vertical structure have been spearheaded by observations of external galaxies and Galactic star counts, respectively. Their results are briefly summarized below.

\section{Radial Density Profiles}

The Sun's location in the disk of the Milky Way is not conducive to direct measurements of the radial distribution of stars. Instead, external spiral galaxies are used as a proxy for determining the general form of stellar radial gradients. Freeman (1970) and de Vaucouleurs \& Pence (1978) showed conclusively that many spiral galaxies are well described by an exponential decline, $\rho(R) \propto \exp -\frac{R}{R_{\circ}}$, where $R_{\circ}$ is the scale length, with typical values of 2-3 kpc (Reid \& Hawley, 2005). This functional form has been applied to the modeling of Milky Way star-counts (e.g. Bahcall \& Soneira, 1980; Siegel et al., 2002; Jurić et al., 2008). There is uncertainty in modern determinations of the radial scale length, with values ranging from 2-6 kpc (Siegel et al., 2002; Robin et al., 2003; Jurić et al., 2008). However, there are promising suggestions of consensus, as scale lengths measured from 2MASS star counts (2800 pc; Ojha, 2001), which should be minimally affected by interstellar extinction, are in good agreement with results from SDSS density maps, measured over a smaller volume with precise photometry (2600 pc; Jurić et al., 2008).

\section{Vertical Density Profiles}

Modern investigations of the Milky Way's vertical stellar density profile can be traced back to Oort (1932) and the Vatican conference (O'Connell, 1958). There, Oort described the gross properties of five different stellar populations, including the thin disk. Furthermore, the ground-breaking star-formation studies of Schmidt (1959, 1963) reinforced the idea of a vertical gradient. Like the radial density law, $\rho(Z)$ is 
usually taken to be an exponential, with the form $\rho(Z) \propto \exp -\frac{|Z|}{Z_{\circ}}$, where $Z_{\circ}$ is the scale height. One of two methods is usually used to measure this value. The first employs absolute magnitude estimates (usually from photometric parallaxes) to determine distances to individual stars and directly measure $\rho(Z)$. This method is subject to incompleteness and Malmquist bias, as well as the uncertainties associated with CMRs. The alternate method, popularized by the seminal study of Bahcall \& Soneira (1980), assumes a luminosity function and Galactic density profile to generate synthetic apparent magnitude-color diagrams ${ }^{8}$. These assumed inputs are then tweaked until the synthetic Hess diagrams match the observed star counts. This method can be computationally intensive, especially if many sightlines need to be examined. Furthermore, there are many assumed input variables, which may produce ambiguous results. Both methods have been employed extensively in the literature, and there is general consensus that the scale height of $\mathrm{M}$ dwarfs is $Z_{\circ} \sim 250-300$ pc (Reid et al., 1997; Siegel et al., 2002; Jurić et al., 2008). There is little evidence of variations of the scale height within the $\mathrm{M}$ dwarf spectral type, and it is not accounted for in this analysis.

Gilmore \& Reid (1983), using star counts to directly measure $\rho(Z)$ in the direction of the Southern Galactic Pole, observed a density profile that changed slope near $Z \sim 1.5 \mathrm{kpc}$. This shift was interpreted as an additional population, the "thick disk". Since this initial investigation, many other studies have confirmed the presence of the thick disk in the Milky Way (e.g., Reid \& Majewski, 1993; Siegel et al., 2002; Jurić et al., 2008, and references therein) and external spiral galaxies (e.g., Yoachim \& Dalcanton, 2006, 2008). The density distribution of the thick disk is generally thought to be exponential in radius and height, with a scale length larger than the thin disk ( 5 kpc; Chen et al., 2001; Larsen \& Humphreys, 2003). The scale height is poorly constrained (Norris, 1999), with smaller values of the scale height corresponding to

\footnotetext{
${ }^{8}$ These color-apparent magnitude diagrams are also known as Hess diagrams, named after the astronomer who introduced them in 1924.
} 
larger local normalizations (see Fig. 1 in Siegel et al., 2002). Typical values range from 600 pc to 1800 pc (e.g., Yoshii, 1982; Buser et al., 1999), with the latest measurements favoring smaller values (Jurić et al., 2008).

Besides the exponential functional form, a sech ${ }^{2}$ formalism has been suggested by Camm $(1950,1952)$ and van der Kruit \& Searle (1981). They demonstrated that an isothermal population produces a sech ${ }^{2}$ disk, which avoids a sharp peak at $Z=0$. The sech ${ }^{2}$ density profile:

$$
\rho(Z)=\rho_{o} \operatorname{sech}^{2}\left(\frac{\mathrm{Z}}{2 \mathrm{Z}_{\mathrm{s}}}\right)
$$

tends to an exponential distribution at large $Z$ (with a scale height of $Z_{s}$ ), but is smooth near $Z=0$.

\subsection{Analysis: Galactic Model Fits and Raw Luminosity Function}

Using the method described above, $(R, Z)$ stellar density maps for each 0.5 mag slice in $M_{r}$ were constructed, from $M_{r}=6.75$ to $M_{r}=17.25$, roughly corresponding to spectral types M0-M8. Some of these maps are shown in Figure 5.11, omitting the lowest luminosity maps, since they only span a few $R, Z$ bins. The bin size in each map is constant, at $25 \mathrm{pc}$ in the $R$ and $Z$ directions. The disk structure is clearly evident in each map, especially at brighter absolute magnitudes $\left(M_{r} \lesssim 10\right)$. For $R, Z$ bins with density errors of $<15 \%$, the following disk density structure is fit:

$$
\begin{gathered}
\rho_{\text {thin }}(R, Z)=\rho_{\circ} f e^{\left(-\frac{R-R_{\odot}}{R_{\circ}, \text { thin }}\right)} e^{\left(-\frac{|Z|-Z_{\odot}}{Z_{\circ} \text { thin }}\right)} \\
\rho_{\text {thick }}(R, Z)=\rho_{\circ}(1-f) e^{\left(-\frac{R-R_{\odot}}{R_{\circ}, \text { thick }}\right)} e^{\left(-\frac{|Z|-Z_{\odot}}{Z_{\circ}, \text { thick }}\right)} \\
\rho(R, Z)=\rho_{\text {thin }}(R, Z)+\rho_{\text {thick }}(R, Z)
\end{gathered}
$$

where $\rho_{\circ}$ is the local density at the solar position $\left(R_{\odot}, Z_{\odot}\right), f$ is the fraction of the total density in the thin disk, $R_{\circ, \text { thin }}$ and $R_{\circ \text {,thick }}$ are the thin and thick disk scale lengths, 
and $Z_{\circ \text {,thin }}$ and $Z_{\circ \text {,thick }}$ are the thin and thick disk scale heights, respectively. Since the density maps are dominated by nearby disk structure, the halo was neglected. Restricting the sample to bins with density errors of $<15 \%$ ensures that they are well-populated by stars and have precise volume measurements, and should accurately trace the underlying Milky Way stellar distribution.

The maps were fit with the standard Levenberg-Marquardt algorithm (Press et al., 1992), using the following approach. First, the thin and thick disk scale heights and lengths, and their relative scaling, were measured from ten absolute magnitude slices, from $M_{r}=7.25-11.75$. Using these relatively luminous stars yields the best estimates for Galactic structure parameters. Including lower-luminosity bins would have biased the fits, artificially shrinking the scale heights and lengths, to compensate for density differences between a small number of adjacent $R, Z$ bins ${ }^{9}$. After the relative normalization and scale heights and lengths are fixed, the local densities are fit for each absolute magnitude slice, using a progressive sigma clipping method similar to Jurić et al. (2008). This clipping technique "guides" the fit, keeping obvious over-densities from biasing the final best-fit. Briefly, a model is computed, and the standard deviation $(\sigma)$ of the residuals is calculated. The $R, Z$ maps are refit, with density points greater than $50 \sigma$ excluded. This process is repeated multiple times, with $\sigma$ smoothly decreasing by the following series: $\sigma=(40,30,20,10,5)$. The resulting Galactic structure parameters $\left(Z_{\mathrm{o}, \text { thin }}, Z_{\mathrm{o} \text {,thick }}, R_{\mathrm{o} \text {,thin }}, Z_{\mathrm{o} \text {,thick }}, f\right)$ are listed in Table 5.3. The "raw" luminosity function, constructed from the local densities of each absolute magnitude slice, is shown in Figure 5.12. These two measurements are the crux of my dissertation, determining fundamental parameters of both the Milky Way and low-mass dwarfs.

In order to produce a final LF and compare my results to previous investigations, the biases inherent in the observations and analysis must be addressed. The raw LF is

\footnotetext{
${ }^{9}$ More simply, you can't measure features 100's of parsecs in length with tracers that are visible to 10's of parsecs, like M8 stars. Or at least you shouldn't believe the answers.
} 
subject to systematics imposed by Nature, such as unresolved binarity, contamination and metallicity gradients, as well as those from the observations and analysis, like Malmquist bias. Furthermore, uncertainties in the CMR can significantly affect the "observed" properties. Analytic corrections for these various systematic effects are not possible with such a complex analysis. Thus, Monte-Carlo realizations of the dataset are employed to test and quantify the effects of multiple biases on the "observed" LF. The creation of the Monte-Carlo catalogs and their application are discussed in the next section. 

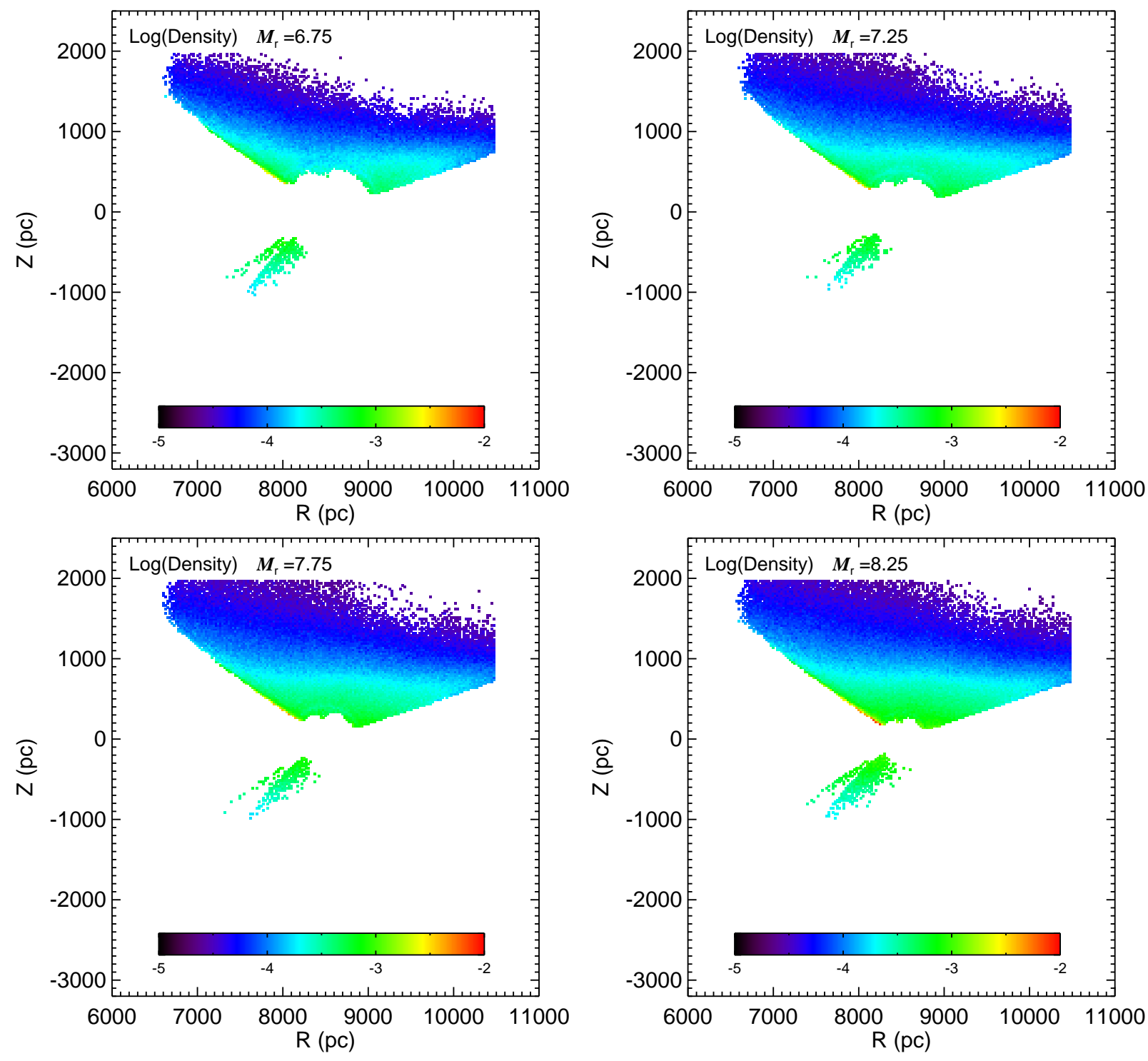

Figure 5.11 Stellar Density Maps from $M_{r}=6.75$ to $M_{r}=8.25$. Note the smooth exponential disk structure in each $(0.5 \mathrm{mag})$ absolute magnitude slice. 

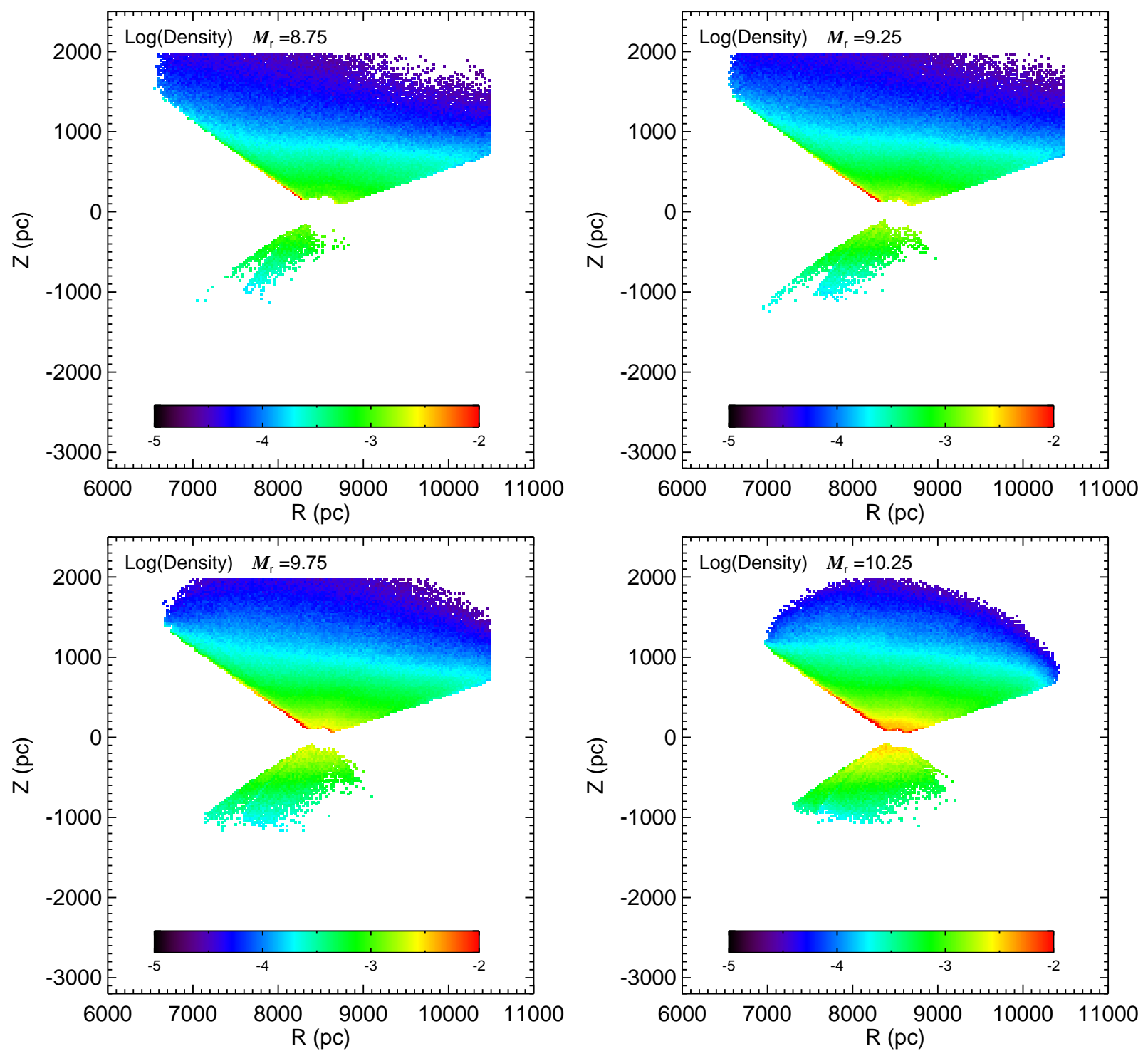

Figure 5.11 Continued. Stellar Density Maps from $M_{r}=8.75$ to $M_{r}=10.25$. 

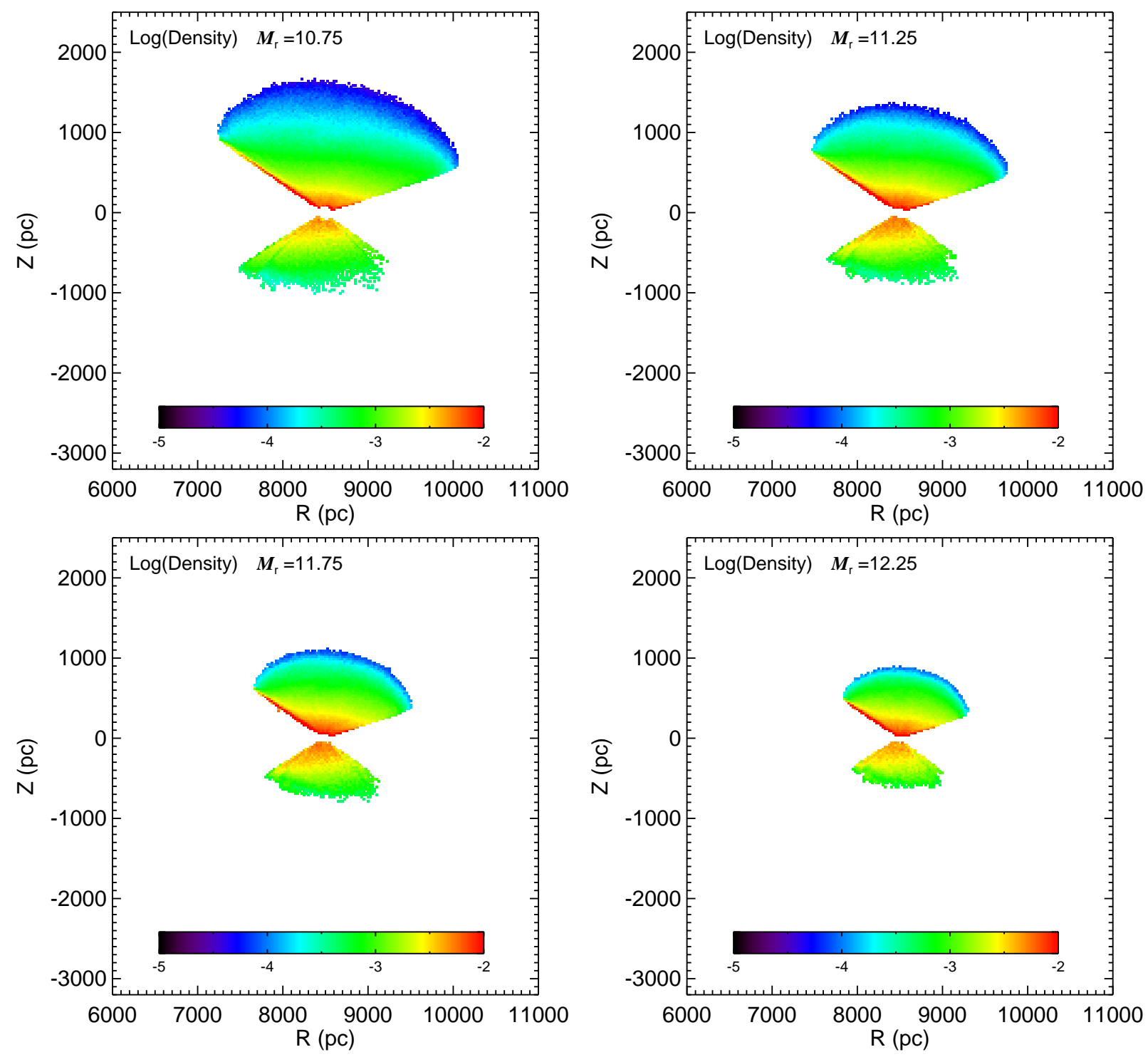

Figure 5.11 Continued. Stellar Density Maps from $M_{r}=10.75$ to $M_{r}=12.25$. 

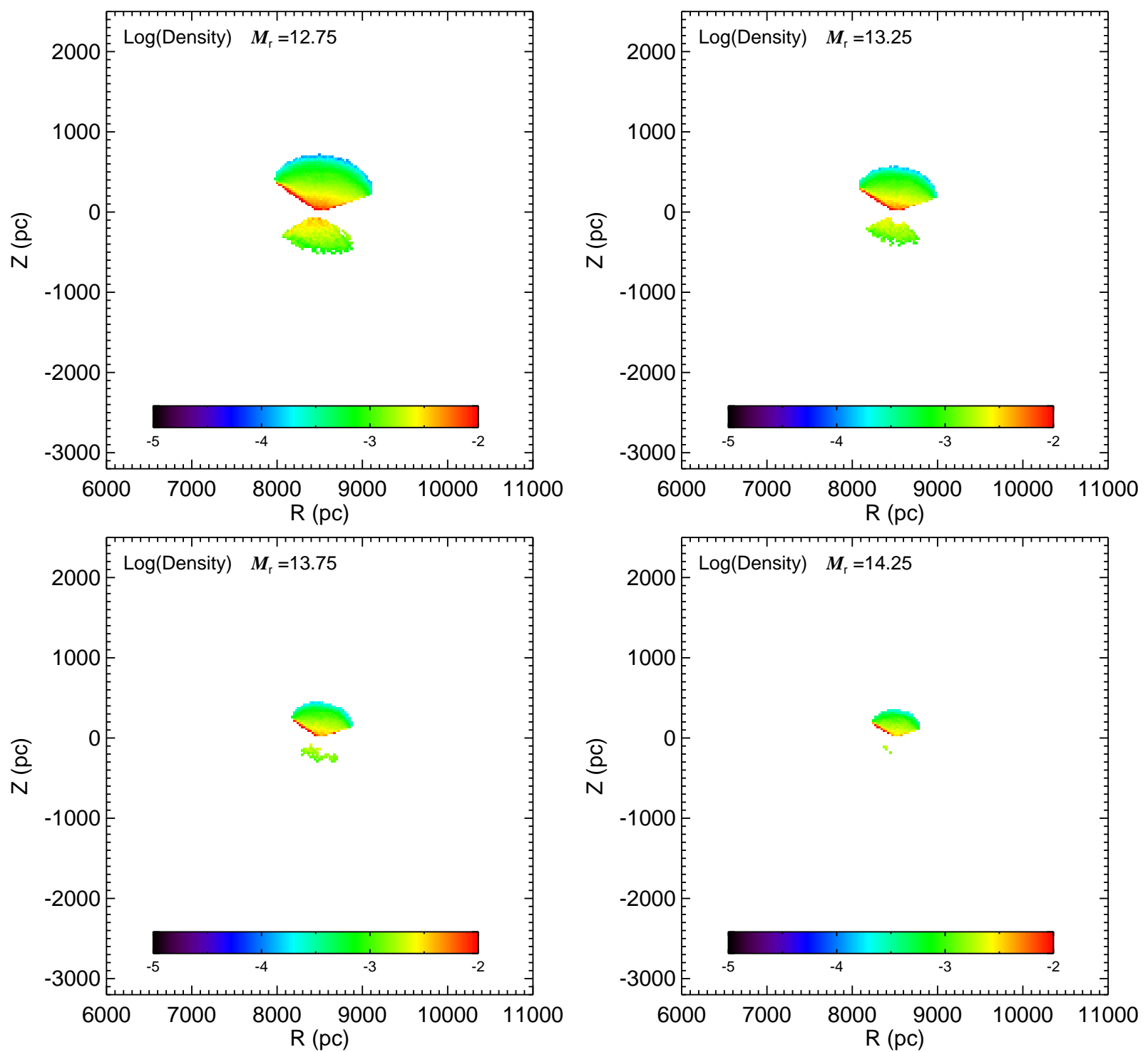

Figure 5.11 Continued. Stellar Density Maps from $M_{r}=12.75$ to $M_{r}=14.25$. 


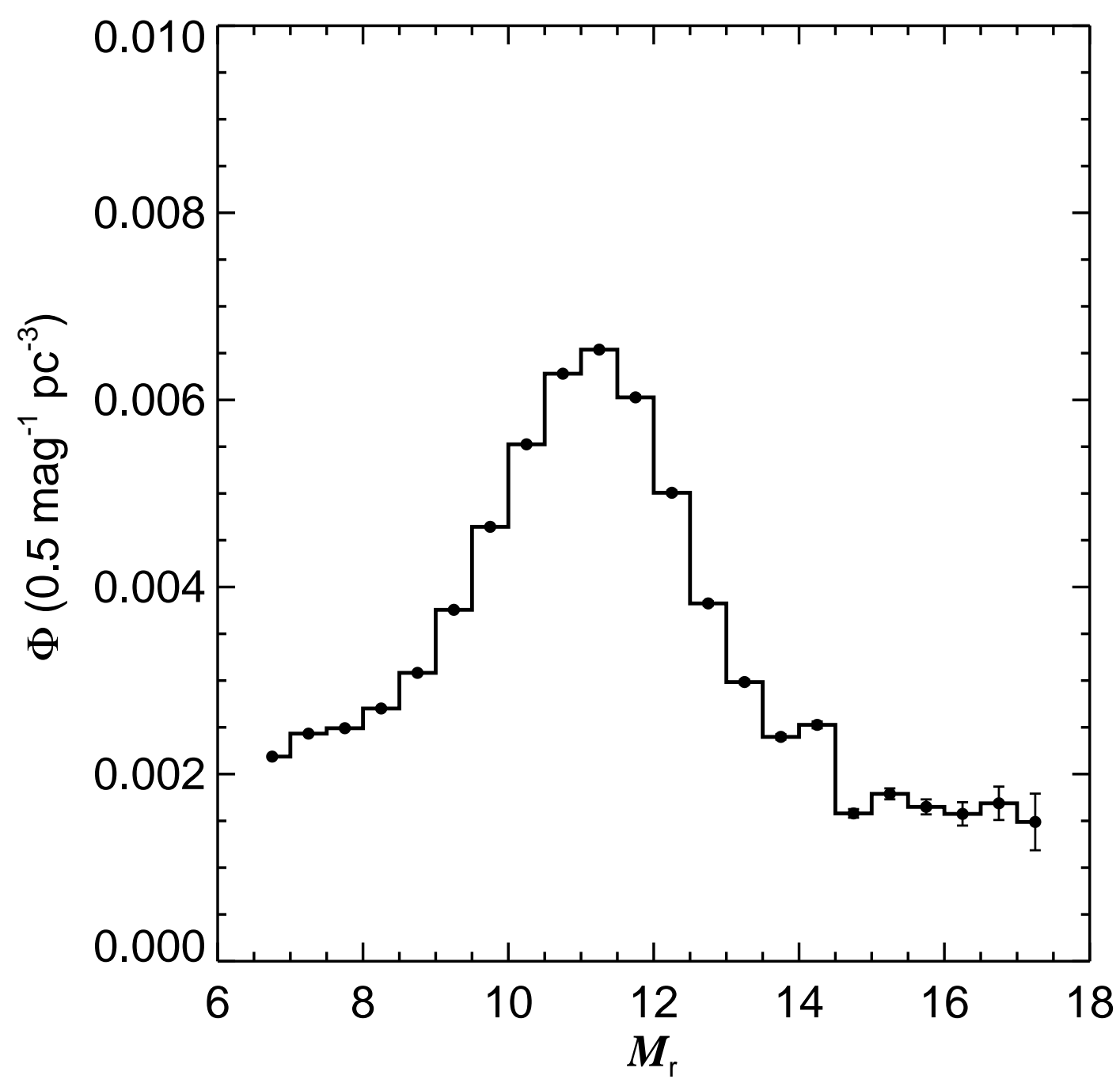

Figure 5.12 Shown is the "raw" $r$-band LF for the stellar sample, using the $\left(M_{r}, r-z\right)$ CMR. Note the smooth behavior, with a peak near $M_{r} \sim 11$, corresponding to a spectral type of $\sim \mathrm{M} 4$. The error bars are the formal uncertainties from fitting the local densities in each $0.5 \mathrm{mag}$ absolute magnitude slice in stellar density.

\subsection{Analysis: Correcting Systematic Errors}

There are a number of biases that systematically affect the construction of the stellar density maps, including Malmquist bias, unresolved binarity, and metallicity gradients. These systematics serve to increase or decrease the measured stellar density 
Table 5.3. Measured Galactic Structure

\begin{tabular}{rll}
\hline \hline Property & Raw Value & Error \\
& & \\
\hline$Z_{\mathrm{o}, \text { thin }}$ & $255 \mathrm{pc}$ & $12 \mathrm{pc}$ \\
$R_{\mathrm{o}, \text { thin }}$ & $2200 \mathrm{pc}$ & $65 \mathrm{pc}$ \\
$Z_{\mathrm{o}, \text { thick }}$ & $1360 \mathrm{pc}$ & $300 \mathrm{pc}$ \\
$R_{\mathrm{o}, \text { thick }}$ & $4100 \mathrm{pc}$ & $740 \mathrm{pc}$ \\
$f$ & 0.97 & 0.006 \\
\hline
\end{tabular}

in a given $R, Z$ bin. To quantify these effects, multiple Monte Carlo catalogs were constructed that mimic the input SDSS observations.

Each Monte Carlo catalog was generated using the positions $(\alpha, \delta)$ from the stellar sample to ensure that the model "stars" would fall within the SDSS footprint. Next, distances were randomly assigned that were consistent with the density distribution along a given line of sight for the assumed Galactic structure parameters. The Galactic scale heights and lengths, along with the thin disk fraction measured from each model are listed in Table 5.4. Absolute magnitudes were selected to be consistent with the observed "raw" LF and colors were assigned to each star using the $\left(M_{r}, r-z\right)$ and $\left(M_{r}, r-i\right)$ CMRs described in Table 4.2. Scatter in absolute magnitude and color was included for the Malmquist bias case. Apparent magnitudes were then computed from the distances and absolute magnitudes.

To estimate the effect of the flag cuts and brightness limits described in $§ 5.2 .2$, two $(r, i-z)$ Hess diagrams were constructed, one from the raw SDSS stellar data, and the other from the stars that remained after the flag cuts described in $§ 5.2 .2$. The two Hess diagrams, along with their ratio, are shown in Figure 5.13. The model stars are statistically "observed", scaling the model $(r, i-z)$ star counts by the ratio of the SDSS $(r, i-z)$ Hess diagrams to simulate the influence of SDSS observations on the model stars. 
Next, each simulated LF is compared to the observed LF (shown in Figure 5.14), to quantify the effects of the bias described below. After exploring how the different systematics affected the output LF, this information was used to "correct" the raw LF, scaling it by the ratio of the input LF to the output model LF.

\subsubsection{Systematics: Fitting Algorithm (The "Clean" Catalog)}

The first Monte Carlo star catalog was generated to examine the density calculation and Galactic structure fits described above. A "clean" catalog was generated, where every star was assumed to be a single star (no binaries) and there was zero dispersion introduced into the absolute magnitude and color. Thus, the color of a model star would exactly trace its absolute magnitude.

The results of fitting this "clean" stellar catalog are listed in Table 5.4. The thin disk scale height which has the largest systematic effect on the measured LF, agrees well with the model inputs. The thick disk scale height and normalization disagree with the model inputs, but I stress that these two properties are highly correlated. Thus, the larger thick disk scale height and smaller normalization contain the same fraction of stellar mass as a thick disk with a larger normalization and smaller scale height (see Figure 1 of Siegel et al., 2002 and Figure 21 of Jurić et al., 2008). Therefore, the large discrepancy between scale heights is not particularly worrisome.

\subsubsection{Systematics: Malmquist Bias}

Malmquist bias (Malmquist, 1936) arises in flux-limited surveys (such as SDSS), when distant stars with brighter absolute magnitudes (either intrinsically, from the known spread in absolute magnitude at a given color, or artificially, due to measurement error) scatter into the survey volume. These stars have their absolute magnitudes systematically overestimated (i.e., are given fainter absolute magnitudes than they actually possess), which leads to underestimated intrinsic luminosities. Thus, their

distances will be systematically short. This effect artificially shrinks the observed 

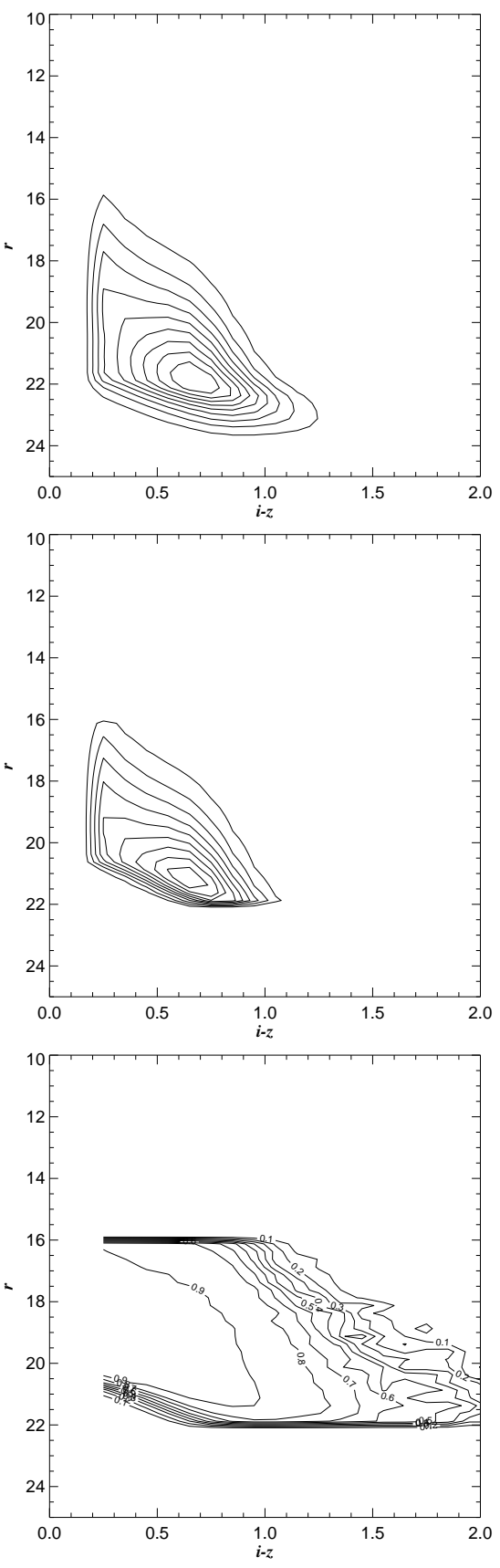

Figure 5.13 Shown are two $(r, i-z)$ Hess diagrams from the SDSS photometry, before (top panel) and after (middle panel) the flag cuts described above. Each contour represents $10 \%$ of the stars. The ratio of the two diagrams (bottom panel) is used to statistically enforce the behavior of SDSS flag and brightness cuts on the Monte Carlo catalogs. Each contour in the bottom panel is labelled, and for most locations in the Hess diagram, the flag cuts preserve $<80 \%$ of the stars. 
scale heights, and inflates the measured LF. Assuming a Gaussian distribution about a "true" mean absolute magnitude $M_{\circ}$, classical Malmquist bias is given by:

$$
\bar{M}(m)=M_{\circ}-\frac{\sigma^{2}}{\log e} \frac{d A(m)}{d m}
$$

where $\sigma$ is the spread in the CMR, $d A(m) / d m$ is the slope of the star counts as a function of apparent magnitude $m$ (see Equation 5.1), and $\bar{M}(m)$ is the observed mean absolute magnitude. Qualitatively, $\bar{M}(m)$ is always less than $M_{\circ}$ (assuming $d A(m) / d m$ is positive), meaning that the observed absolute magnitude distribution is skewed towards more luminous objects.

Classical Malmquist bias only accounts for scatter in absolute magnitude at a given color, and does not account for random photometric errors, which moves stars between adjacent absolute magnitude (or color) bins in the LF. "Continuous" Malmquist bias was first characterized by Stobie et al. (1989), who demonstrated that the classical Malmquist correction was a biased estimator of the true LF. While random photometric errors scatter stars between different bins, the underlying LF is not uniformly distributed. Therefore, color errors will act to smooth out peaks in the LF and fill in under-densities. Continuous Malmquist bias is described by:

$$
\frac{\Delta \Phi}{\Phi}=\frac{1}{2} \sigma^{2}\left[\left(\frac{0.6}{\log e}\right)^{2}-\left(\frac{1.2}{\log e}\right) \frac{\Phi^{\prime}}{\Phi}+\frac{\Phi^{\prime \prime}}{\Phi}\right]
$$

Fortunately, random errors in SDSS photometric colors are small $(\lesssim 4 \%$, Padmanabhan et al., 2008), so the scatter between LF bins should be minimal.

Malmquist bias effects were quantified by producing a model stellar catalog with scatter in absolute magnitude and color about the mean predicted by the $\left(M_{r}, r-z\right)$ CMR. An absolute magnitude dispersion of $\sigma_{M_{r}}=0.4 \mathrm{mag}$ and a color dispersion of $\sigma_{r-z, r-i}=0.04$ mag was assumed, in agreement with the observed scatter in the color-magnitude diagrams (see Table 4.2). As mentioned above, Malmquist bias acts 
to artificially shrink distances, decreasing the measured scale heights and increasing the measured LF. This is most prevalent at higher luminosities $\left(M_{r} \sim 7\right)$, where the differences range up to $\sim 35 \%$. The LF measured with the Malmquist bias model is shown in Figure 5.14. The largest effects are seen for the brighter stars. These stars are more common in the SDSS sample (see Figure 5.5) and span a larger volume. Thus, they are more susceptible to having over-luminous stars scattered into their absolute magnitude bins.

\subsubsection{Systematics: Unresolved Binarity}

For all but the closest stars, binaries will masquerade as a single star. The unresolved pair will be over-luminous at a given color, leading to an underestimate of their distance. This compresses the density maps, leading to decreased scale heights and lengths, as more "stars" are piled up at smaller distances. Thus, the scale heights and lengths measured with the SDSS photometry will be systematically underestimated.

Currently the parameter space that describes binaries: binary fraction, mass ratio, and average separation, is not well constrained for $\mathrm{M}$ dwarfs. However, there are some general trends that are useful for modeling their gross properties. First, the binary fraction seems to steadily decline from $\sim 50 \%$ at $\mathrm{F}$ and $\mathrm{G}$ stars (Duquennoy \& Mayor, 1991) to about 30\% for M dwarfs (Fischer \& Marcy, 1992; Delfosse et al., 2004; Lada, 2006). Next, the mass ratio distribution becomes increasingly peaked towards unity at lower masses. That is, F and $\mathrm{G}$ stars are more likely to have a companion from a wide range of masses, while $\mathrm{M}$ dwarfs are commonly found with a companion of nearly the same mass, when the $\mathrm{M}$ dwarf is the primary (warmer) star (Burgasser et al., 2007). The average separation distribution is not well known, but many companions are found with typical separations of $\sim 10-30$ AU (Fischer \& Marcy, 1992), while very-low mass stars have smaller average separations (Burgasser et al., 2007). At the typical distances probed by SDSS (100s of pc) combined with the average width of the point spread function $\left(1.4^{\prime \prime}\right.$ in $\left.r\right)$, these binary systems would 
be unresolved in SDSS photometry.

Following the methodology of Covey et al. (2008), the effects of unresolved binarity are included by introducing a mass dependent binary fraction $f_{m}$ in the Monte Carlo model, which decreases linearly with the following form:

$$
f_{m}(\mathrm{M})=0.45-\frac{0.7-\mathrm{M}_{p}}{4}
$$

where $\mathrm{M}_{p}$ is the mass of the primary star. This simple relation follows observed trends, decreasing from $\sim 50 \%$ at $\mathrm{M}=1 \mathrm{M}_{\odot}$ (Duquennoy \& Mayor, 1991; Reid \& Gizis, 1997) to $\sim 30 \%$ at $\mathrm{M}=0.1 \mathrm{M}_{\odot}$ (Delfosse et al., 2004; Lada, 2006; Burgasser et al., 2007). The luminosity of a companion is chosen from the input LF, with the condition that the secondary is less luminous than the primary. This semi-independent sampling mimics the observed distribution of binary mass ratios (Duquennoy \& Mayor, 1991; Reid \& Gizis, 1997; Burgasser et al., 2007) with mass ratio distributions that are flat at large masses, and peak near unity at small masses.. The separation between the two components is not simulated, as the overwhelming majority of binaries in actual SDSS observations will be unresolved over the distances probed. Finally, the flux from each component is summed and a combined apparent magnitude and color is computed.

Note that the simulations are restricted to binary pairs. Higher order multiples are rare in the solar neighborhood (Reid et al., 2002; Shkolnik et al., 2008), so they are neglected in this analysis.

As qualitatively explained above, the main effect of unresolved binarity is to decrease scale heights. The thin disk scale height measured for the thin disk is $80 \%$ smaller than the model input, which produces an unrealistic spike in the luminosity function. Thus, the scale height was fixed at 240 parsecs, and the resulting LF is shown in Figure 5.14. With a fixed scale height, the relative effect of binarity is clearer. Unresolved binarity produces over-luminous stars (similar to Malmquist 
Table 5.4. Monte Carlo Galactic Structure Results

\begin{tabular}{rllllll}
\hline \hline \multicolumn{1}{c}{ Model } & $Z_{\mathrm{o}, \text { thin }}(\mathrm{pc})$ & $R_{\mathrm{o}, \text { thin }}(\mathrm{pc})$ & $Z_{\mathrm{o}, \text { thick }}(\mathrm{pc})$ & $R_{\mathrm{o}, \text { thick }}(\mathrm{pc})$ & $f$ & $\chi^{2}$ \\
\hline Input & 240 & 2200 & 900 & 4700 & 0.94 & $\ldots$ \\
Clean & 235 & 1750 & 1910 & 9624 & 0.98 & 3.74 \\
Malmquist Bias & 234 & 1690 & 1390 & 6770 & 0.98 & 3.41 \\
Unresolved Binaries & 190 & 1440 & 980 & 5800 & 0.97 & 4.43 \\
Combined & 190 & 1400 & 840 & 4500 & 0.96 & 3.99 \\
\hline
\end{tabular}

Table 5.5. Best Fit Galactic Structure

\begin{tabular}{rllll}
\hline \hline Property & Raw Value & Error & Bias-Corrected Value & Error \\
& & & & \\
\hline$Z_{\text {o,thin }}$ & $255 \mathrm{pc}$ & $12 \mathrm{pc}$ & 320 & $10 \%$ \\
$R_{\mathrm{o}, \text { thin }}$ & $2200 \mathrm{pc}$ & $65 \mathrm{pc}$ & 2750 & $20 \%$ \\
$Z_{\mathrm{o}, \text { thick }}$ & $1360 \mathrm{pc}$ & $300 \mathrm{pc}$ & 1400 & $30 \%$ \\
$R_{\mathrm{o}, \text { thick }}$ & $4100 \mathrm{pc}$ & $740 \mathrm{pc}$ & 4500 & $30 \%$ \\
$f$ & 0.97 & 0.006 & 0.98 & $10 \%$ \\
\hline
\end{tabular}

bias), which shrinks the observed Galactic structure, moving stars closer, and inflating local densities. At the bright end of the LF, there is hardly any difference between the Malmquist bias and unresolved binarity cases. At these luminosities, the binary is dominated by the more luminous star, and doesn't brighten significantly. However, at low luminosities, a binary system is more likely to be composed of equal mass stars. This produces the largest increase in brightness, resulting in large shifts relative to the input LF. The effects of unresolved binarity are listed in Table 5.4 as well as the "combined case" (Malmquist bias and unresolved binaries). 


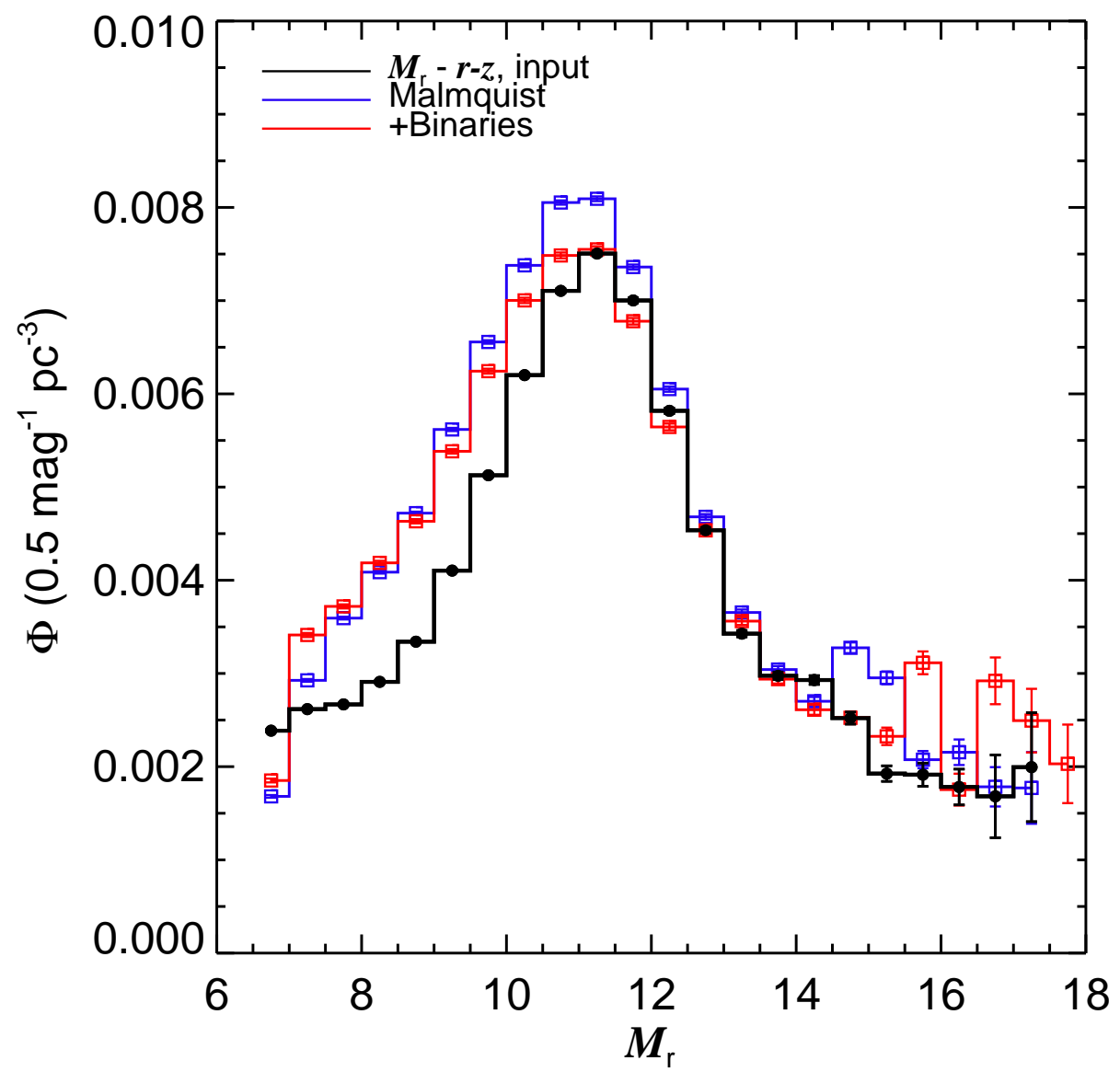

Figure 5.14 Shown are the derived luminosity functions for the Malmquist bias Monte Carlo model with (red line) and without (blue line) binaries included. The largest effect is seen at large luminosities, where the number of stars observed is larger. At the faint end, the density of binaries is over-estimated, since many binary systems at these luminosities are composed of equal mass stars. This artificially shrinks their distances, increasing the local density. 


\subsubsection{Other Systematics}

The color-magnitude relation (CMR) is the dominant source of systematic error in this analysis. Errors in the assumed form of the CMR directly impact the derived stellar density maps, as well as the relative absolute magnitude populations. Below, these systematic effects are quantified, using the raw SDSS observations. First, the systematic changes due to using different CMRs are explored. Next, uncertainties due to metallicity are quantified, and the effect on the LF and Galactic structure parameters are shown. Finally, extinction and contamination from $\mathrm{M}$ giants and quasars are discussed.

\section{Other Systematics: Color-Magnitude Relations}

As stated above, the colors of the model stars are assigned with the $\left(M_{r}, r-z\right)$ and $\left(M_{r}, r-i\right)$ relations from Table 4.2. Thus, applying these relations to the Monte Carlo models is not a true test of the systematic effects on the analysis. Instead, the $\left(M_{r}, r-i\right)$ was applied to the raw stellar catalog, and compared to the raw LF, which was measured with the $\left(M_{r}, r-z\right) \mathrm{CMR}$, in Figure 5.15. The changes in Galactic structure parameters are given in Table 5.6. The shapes of the resulting LFs are similar. This result is promising, demonstrating that the $\left(M_{r}, r-i\right)$ and $\left(M_{r}, r-z\right)$ CMRS give consistent results.

To further explore the systematics induced by the CMR, the $\left(M_{r}, r-z\right)$ relation was shifted by \pm 0.5 mags in $M_{r}$, nearly twice the measured dispersion. The

observed data were fit with these relations. The differences compared to the "raw" LF are shown in Figure 5.16, and the measured Galactic profile is listed in Table 5.6. These shifts in the CMR are the "worst case" representing the extreme limits of systematic errors in the analysis. The red histogram clearly demonstrates an increase in the local density. These stars were artificially dimmed by 0.5 absolute magnitudes, moving them closer to the Sun. The Galactic model fit compensates by increasing 


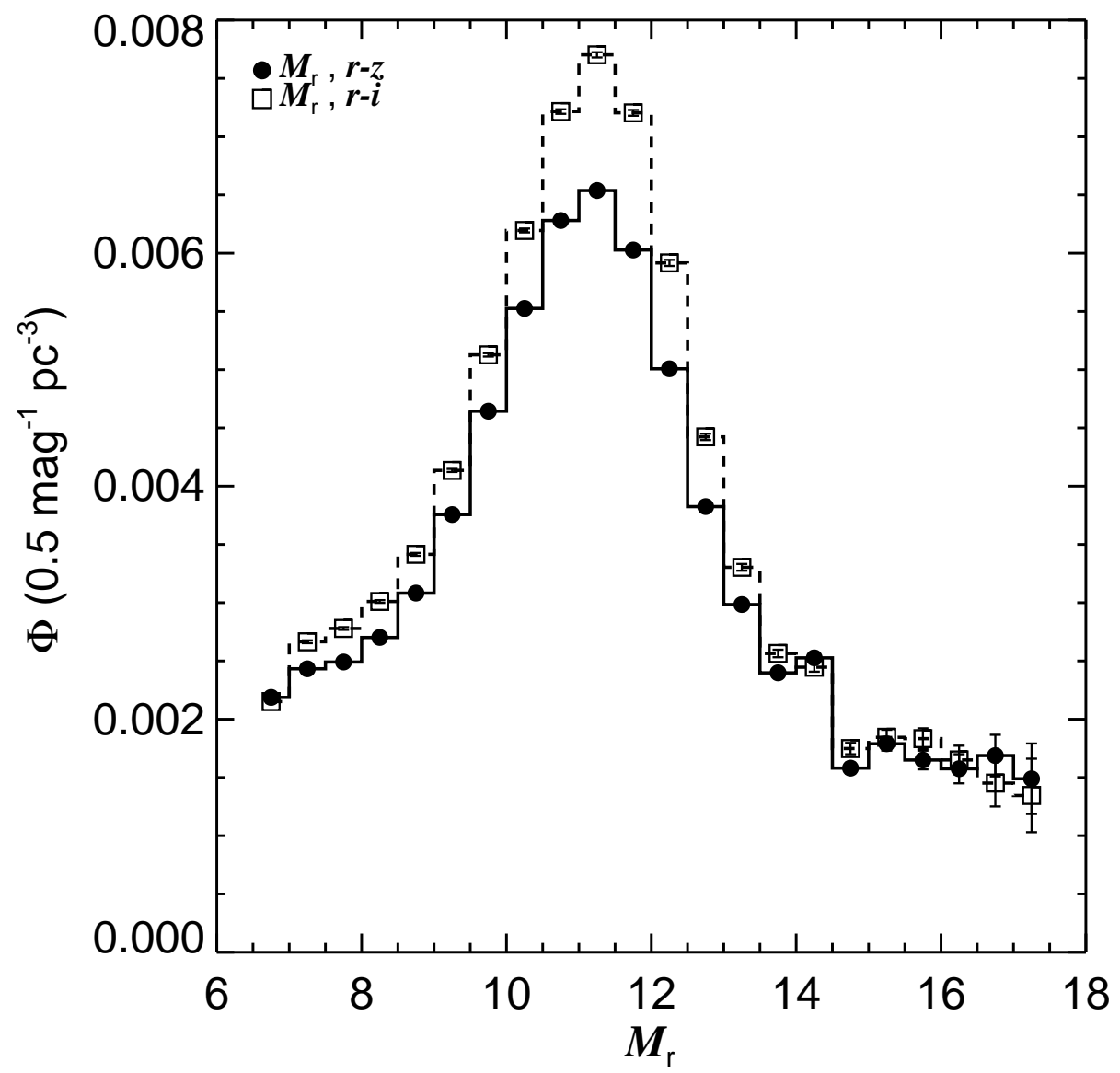

Figure 5.15 Shown are the LFs measured from density maps computed with the $\left(M_{r}, r-z\right)$ (filled circles) and $\left(M_{r}, r-i\right)$ (open squares) CMRs. Their broad agreement in shape confirms that the CMRs used in this analysis are consistent. The differences from $M_{r} \sim 10-12$ are representative of the true uncertainty in the LF. 
the local density, and decreases the scale heights. The increase in local density is the dominant effect, since most of the stars in the skewed sample are nearby. To study the opposite effect, stars were artificially brightened by 0.5 absolute magnitudes, and the corresponding LF is shown as the blue line in Figure 5.16. The distances are now scaled up, with an increase in density at larger distances. The model fits respond accordingly. First, there are relatively less stars are nearby distances, so a large local density is not needed. Next, the falloff at intermediate distances is less severe than the "raw" case, so the thick disk fraction increases, offsetting the decrease in the thick disk scale height. Note that the normalization of the thick disk and the its scale height are highly correlated, with large scale heights normally coupled with small local normalizations (Siegel et al., 2002).

This degeneracy can introduce counter-intuitive results, such as the one explained above. When distances are overestimated (absolute magnitudes brightened by 0.5 mags), the scale heights should increase compared to the fiducial "raw" case. Yet, this is not the best-fit solution, as both the thin and thick disk scale heights decrease, while the thick disk normalization increases from $4 \%$ to $10 \%$. In the best-fit solution, more stars are placed in this puffed-up component, which mimics an increase in scale height. To further clarify this argument, two models were computed with the thin disk fraction held fixed at $96 \%$, consistent with the measured ratio. The results are shown in Table 5.6. When this scaling is fixed, the intuitive scale heights are recovered from the data. In the case of overestimated distances, larger scale heights are found, while smaller scale heights are measured for underestimated distances. This example highlights the complicated interdependencies of this analysis. Finally, the effect of normalization on the resulting LF is shown in Figure 5.16. Fortunately, the differences between the LFs measured with fixed and varying normalizations are small, indicating that the main thrust of this project, the LF, is a robust result. 


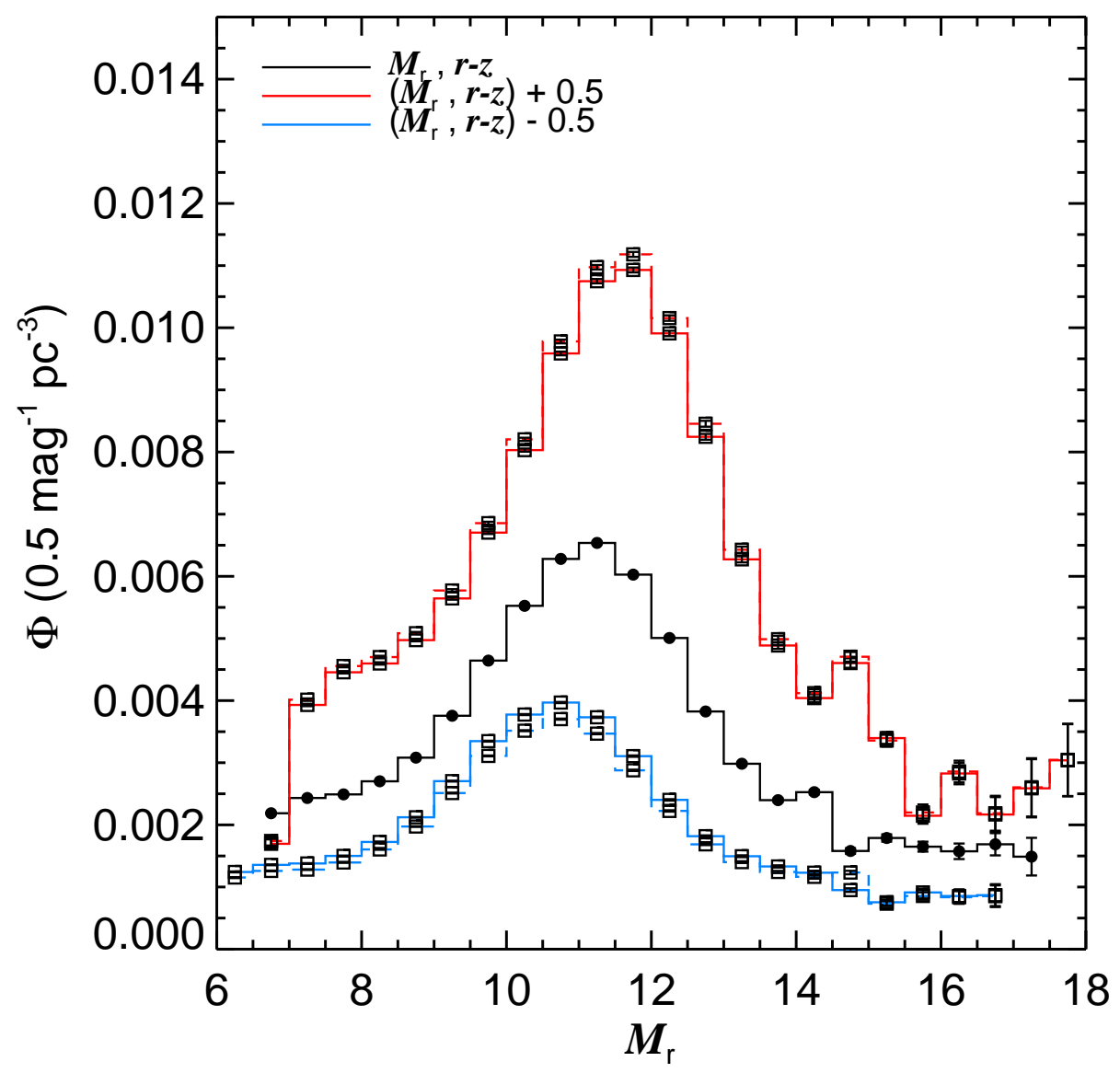

Figure 5.16 Shown are the systematic changes in the LF induced by shifting the $\left(M_{r}, r-z\right)$ CMR by +0.5 (red line) and -0.5 (blue line) mags in $M_{r}$, together with the raw LF. The dashed histograms correspond to a thin disk fraction held fixed at 0.96. Note that the shift in absolute magnitude moves the peak of the LF to higher or lower luminosities, corresponding to the sign of the shift. Furthermore, holding the normalization fixed does not induce large relative changes in the resulting LFs, as the solid and dashed histograms are virtually indistinguishable. 


\subsubsection{Other Systematics: Metallicity}

The metallicity of a star strongly affects the radiative transfer through its atmosphere. Low metallicity leads to less opacity and a higher temperature for a star of a given mass. This shifts the star to a higher luminosity and temperature compared to its solar-metallicity counterpart. The effect produces a subdwarf "main sequence" which lies below ${ }^{10}$ the solar-metallicity main sequence. Thus, at a given color, stars with lower metallicities have larger (fainter) absolute magnitudes. Failing to account for this effect artificially brightens low-metallicity stars, increasing their actual distance. This inflates densities at large distances, increasing the observed scale heights (King et al., 1990).

Quantifying the effects of metallicity on low-mass dwarfs is complicated by multiple factors. First, direct metallicity measurements of these cool stars are difficult, as current models do not accurately reproduce their complex spectral features. Molecular line lists are incomplete, and thus the opacity is not correctly calculated. Currently, measurements of metallicity-sensitive molecular bandheads $(\mathrm{CaH}$ and $\mathrm{TiO})$ are used to estimate the metallicity of $\mathrm{M}$ dwarfs at the $\sim 1$ dex level (see Gizis, 1997; Lépine et al., 2003b; Burgasser \& Kirkpatrick, 2006; West et al., 2008), but detailed measurements are not available for single stars. Unfortunately, even this crude estimator is not applicable to stars with only broadband photometry.

Furthermore, the effects of metallicity on the absolute magnitude of low-mass stars are poorly known. Accurate parallaxes for nearby subdwarfs do exist (Monet et al., 1992; Reid, 1997; Burgasser et al., 2008), but determining their metal abundances is hindered by the effects described above. Observations of clusters with known metallicities could mitigate this problem (Clem et al., 2008), but there are no comprehensive observations in the ugriz system that probe the lower main sequence. Thus, extrapolations from brighter cluster members are used to determine a relation between the

${ }^{10}$ Actually, blue-ward and towards higher luminosities, as first described by Sandage \& Eggen (1959). 
metallicity and offset in absolute magnitude $\left(\Delta M_{r}\right)$. This extrapolation is probably incorrect, since the subdwarf sequence actually crosses over the main-sequence at very red colors (Reid \& Hawley, 2005), indicating that a simple offset in absolute magnitude is not sufficient. However, to test the systematic effects of metallicity on this study, the $\left([\mathrm{Fe} / \mathrm{H}], \Delta M_{r}\right)$ relation from Ivezic et al. (2008) was adopted, but I note that this relation is appropriate for more luminous $\mathrm{F}$ and $\mathrm{G}$ stars, near the main-sequence turnoff.

As mentioned above, an assumed metallicity gradient in the Galaxy will systematically affect the derived stellar densities. Stars at large vertical distances are assumed to be metal poor, and will lie blue-ward of the main sequence at a given absolute magnitude. To account for this effect, a metallicity gradient with respect to the Plane is assumed along with an offset in absolute magnitude determined empirically from ugriz observations of clusters (Clem, 2006; Ivezic et al., 2008). The adopted metallicity gradient is:

$$
[F e / H]=-0.0958-0.000277|Z|
$$

At small Galactic heights $(Z \lesssim 100 \mathrm{pc})$, this linear gradient produces a metallicity of about $[\mathrm{Fe} / \mathrm{H}]=-0.1$, appropriate for nearby, local stars (Allende Prieto et al., 2004). At a height of $\sim 2 \mathrm{kpc}$ (the maximum height probed by this study), the metallicity is $[\mathrm{Fe} / \mathrm{H}] \sim-0.65$, consistent with measured distributions (Ivezic et al., 2008). The actual metallicity distribution is probably more complex, but given the uncertainties associated with the effects of metallicity on $\mathrm{M}$ dwarfs, a simple form is preferred. The correction to the absolute magnitude, $\Delta M_{r}$, measured from clusters of known metallicity and distance (Ivezic et al., 2008), is given by:

$$
\Delta M_{r}=-0.10920-1.11[\mathrm{Fe} / H]-0.18[\mathrm{Fe} / H]^{2}
$$

Substituting Equation 5.14 into Equation 5.15, yields a quadratic $\left(\Delta M_{r}, Z\right)$ in 
Galactic height. After initially assigning absolute magnitudes and distances with the CMRs appropriate for nearby stars, the estimated height above the Plane, $Z_{\text {ini }}$ is estimated. This is related to the actual height, $Z_{\text {true }}$ through the following equation:

$$
Z_{\text {true }}=Z_{\text {ini }} 10^{\frac{-\Delta M_{r}\left(Z_{\text {true }}\right)}{5}}
$$

The true height above the Plane is calculated by finding the root of this non-linear equation. Since $\Delta M_{r}$ is a positive value, the actual distance from the Plane, $Z_{\text {true }}$ is smaller than the initial estimate, $Z_{\text {ini }}$. As explained above, this effect becomes important at larger distances, moving stars inwards and decreasing density gradients. Thus, if metallicity effects are neglected, the scale heights and lengths are overestimated.

In Figure 5.17, the systematic effects of metallicity dependent CMRs are shown. The first is the extreme limit, where all stars in the sample have an $[\mathrm{Fe} / \mathrm{H}] \sim-0.65$, corresponding to a $\Delta M_{r}$ of roughly 0.5 magnitudes. This effect was examined above in §5.5.4. Next, shows the effect of the metallicity gradient given in Equation 5.14, with (solid yellow line) and without (solid blue line) a fixed thin disk fraction. As in the discussion of systematic CMR differences, the derived LF is a robust result, despite the differences in the derived Galactic structure parameters. Note that local densities are increased, since more stars are shifted to smaller distances.

\subsubsection{Other Systematics: Extinction}

Interstellar extinction systematically affects the brightness and colors of the stars, making the observations dimmer and redder than the intrinsic starlight. Since the photometry in this study is centered on the Northern Galactic Cap, interstellar extinction does not strongly affect the sample. SDSS provides corrections in the ugriz system, computed using the Schlegel et al. (1998) extinction maps and an assumed dust law with $R_{V}=3.1$. For this sample, the median extinction is $A_{r}=0.09$, while $95 \%$ of the sample has $A_{r}<0.41$. Typical absolute magnitude differences range up 


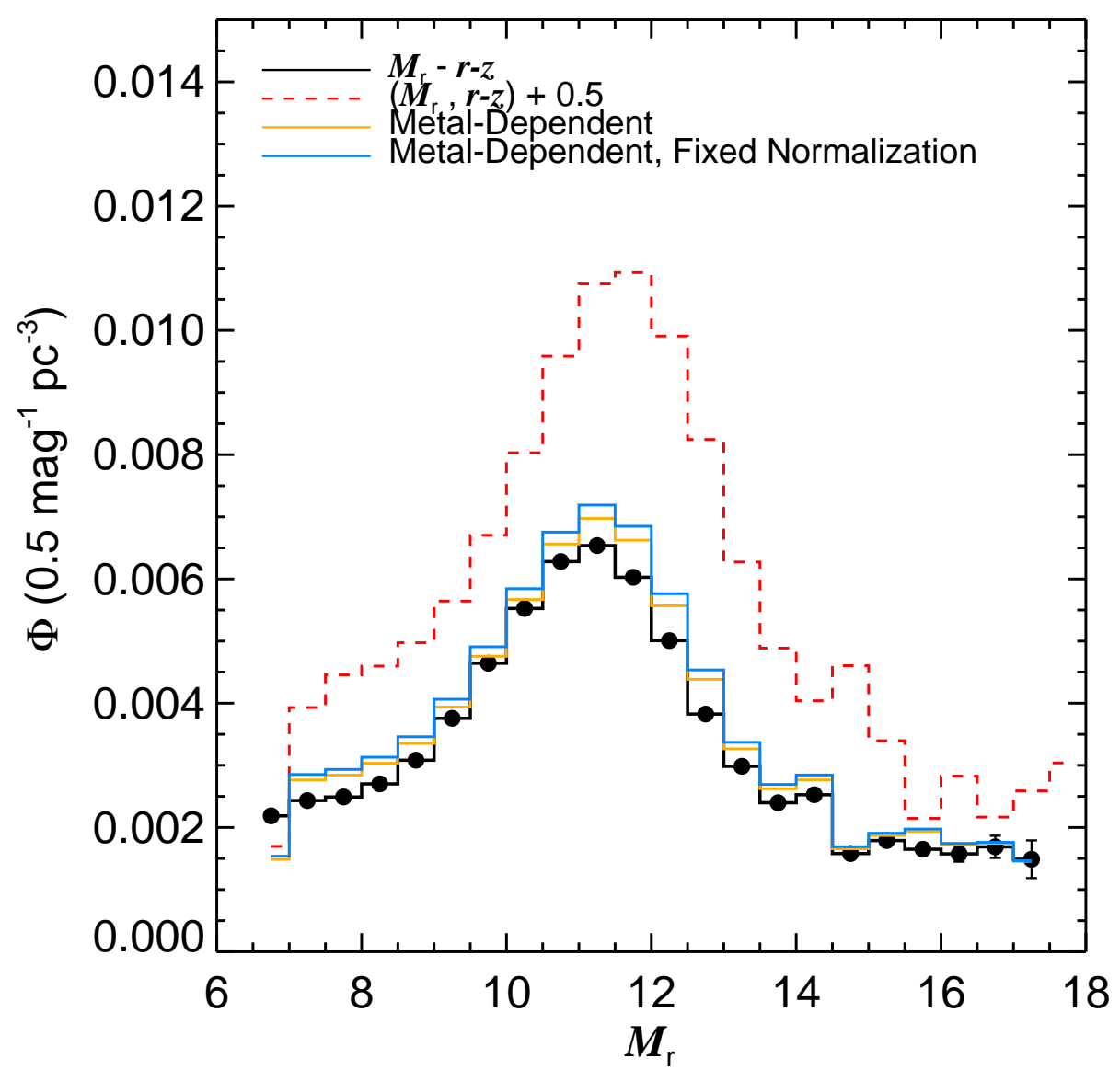

Figure 5.17 Shown are the differences in the LF induced by metallicity gradients, along with the raw LF (black line). The red histogram corresponds to the extreme limit, where all stars are metal-poor $([\mathrm{Fe} / \mathrm{H}] \sim-0.65)$. The yellow histogram shows the effect of the metallicity gradient from Equation 5.14, without fixing the thin disk / thick disk normalization. The blue histogram shows the measured LF with a metallicity dependent CMR and a fixed thin disk fraction $(f=0.96)$. 
to $\sim 1$ magnitude, producing distance corrections of $\sim 40 \mathrm{pc}$, enough to move stars between $R, Z$ bins.

Given the relative faintness of stars observed by SDSS, many of them should be located at distances beyond the dust layer, which is thought to have a scale height of $\sim 15$ pc. Thus, I apply the full extinction corrections to compute the "raw" LF. In Figure 5.18, the effects of omitting the extinction and reddening corrections are shown. The effect is largest at brighter absolute magnitudes. Without correcting for extinction and reddening, stars are observed to be fainter and redder. The change in color results in a larger change in absolute magnitude relative to the dimming of the apparent magnitude. The net effect decreases the distance modulus, moving stars closer and increasing the local density. Globally, this is a small effect $(<6 \%)$, with a maximum of $20 \%$ in some bins.

\subsubsection{Other Systematics: Contamination}

As the current sample is composed solely of photometry, it is subject to contamination from objects that appear to be low-mass stars, but are not. In particular, M giants and quasars are likely to be the largest contributors to contaminants in the sample Covey et al. (2008). Fortunately, I have reason to suspect that these objects will not seriously impact the results. First, given the bright and faint limits of SDSS, the nearest M giant would be found at distances $\sim 100 \mathrm{kpc}$, where intrinsic densities will be negligible. Moreover, the higher metallicities needed to produce M giants would further limit their surface densities (Reid et al., 1997). Quasars, thought to be fueled by massive black-holes in the early Universe, have redshifted colors that mimic the reddest stars in the sample. Fortunately, only the highest redshifts $(>4)$ will overlap the $\mathrm{M}$ dwarf $(r-i, i-z)$ color locus. These are the rarest quasars, with only 1,000 expected for the entire SDSS survey (Fan, 1999).

Yet, without spectroscopy of each object in the sample to verify its true nature, some contamination will always be present. While acquiring spectra of over 15 million 


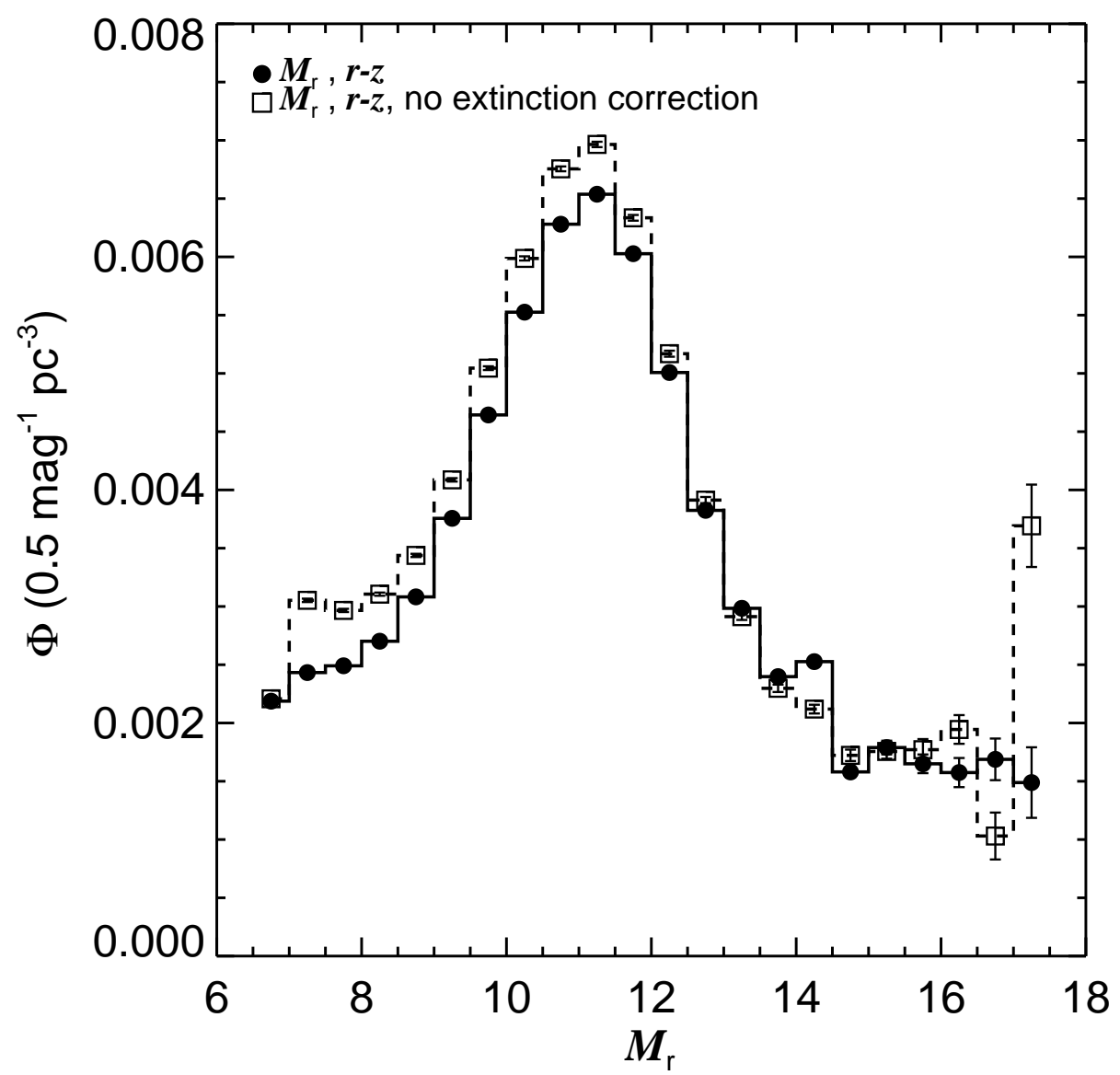

Figure 5.18 Shown is the systematic effect of extinction on the raw LF. When no extinction correction is applied (open squares), distant stars act to inflate the local densities of the brightest stars, compared to the fiducial case (filled circles). At fainter luminosities, this effect becomes less important. 
stars is not currently feasible, Covey et al. (2008) acquired $\sim 10,000$ spectra over 30 sq. deg., with complete follow-up of all red point sources in a smaller 1 sq. deg. subfield. They quantified the contamination rate of the photometric sample, with quasars composing $<0.3 \%$ of red point sources, and $\mathrm{K}$ and $\mathrm{M}$ giants having a global contamination rate of $<5 \%$. These contamination rates are more important for their study, since it was focused along one line of sight. Since M giants and quasars will be overwhelmed by $\mathrm{M}$ dwarfs, they will have a minimal impact on the model fits and not skew the local densities.

\subsubsection{Systematic Uncertainties}

While the statistical error in each LF bin is quite small, typically $\lesssim 0.1 \%$, it does not represent the major source of uncertainty in this analysis. The assumed CMR dominates the systematic uncertainty, affecting the shape of the LF and resulting MF. In order to quantify the statistical uncertainty in the LF and Galactic structure, the following procedure was employed. The LF was computed five times using different CMRs: The $\left(M_{r}, r-z\right)$ and $\left(M_{r}, r-i\right)$ CMRs with and without metallicity corrections, and the $\left(M_{r}, r-z\right)$ CMR without correcting for Galactic extinction. Each LF was corrected for Malmquist bias and binarity. An example of this correction is shown in Figure 5.19. The chosen CMRs should be representative of the major sources of uncertainty in the analysis. The weighted mean of each LF bin and Galactic structure parameter was computed. The weights were estimated as $1 /\left(\chi^{2}\right)^{2}$, which should track the relative quality of each fit. Furthermore, this weighting scheme should prevent LFs and Galactic structure parameters that do not fit the data well from biasing the final outcome. The uncertainty in each LF bin was set by the maximum and minimum at each point, often resulting in asymmetric error bars. This uncertainty is propagated through the entire analysis pipeline through by using three LFs: the weighted mean, the "maximum" LF, corresponding to the maximum in each local density bin, and the "minimum" LF, corresponding to the lowest observed values 
for the LF. The maximum and minimum LFs set the uncertainty throughout the remainder of the analysis. The LFs measured by each CMR are plotted in Figure 5.20, along with the mean, bias-corrected LF. The LF is given in Table 5.8.

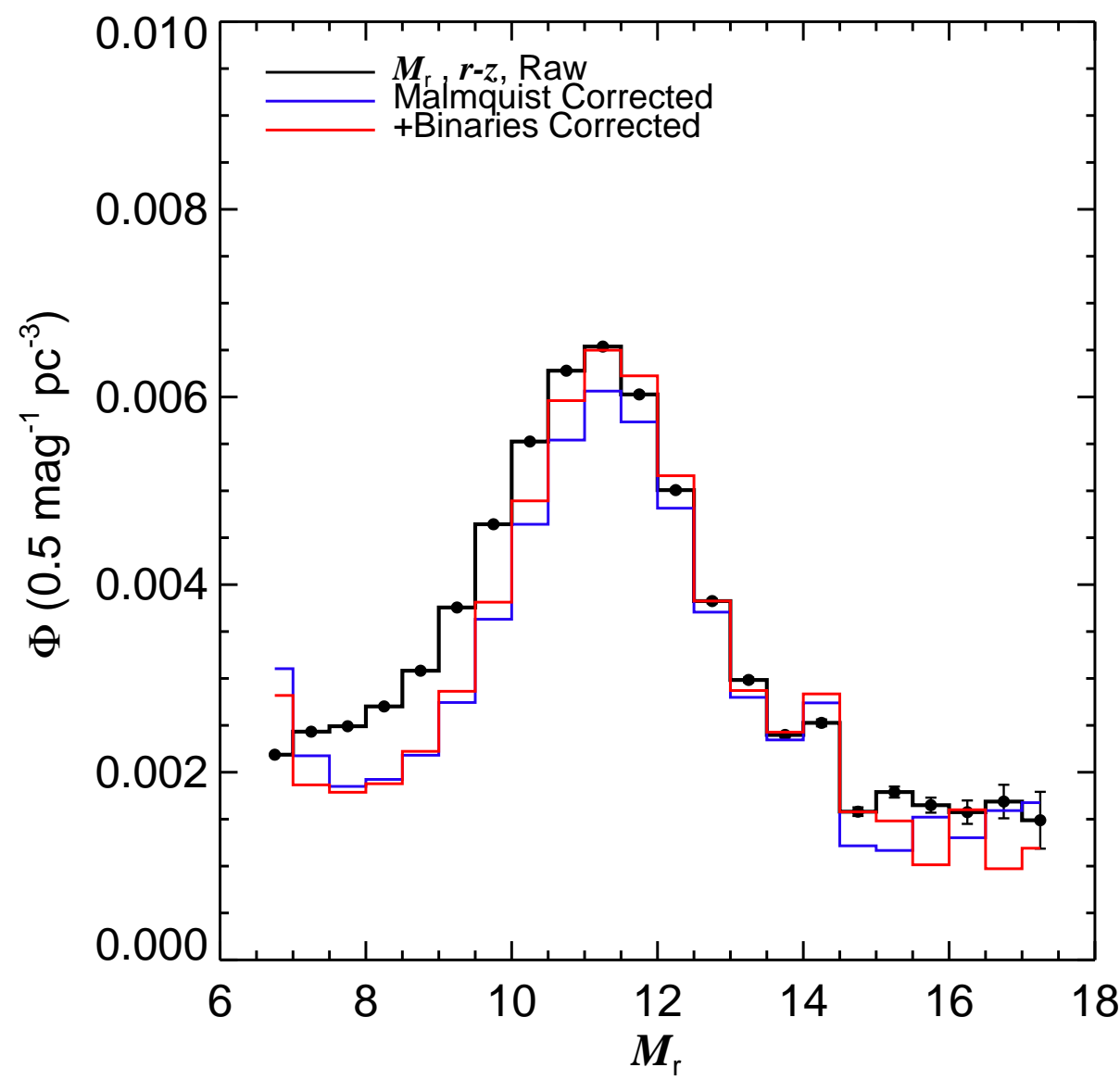

Figure 5.19 Shown is the raw LF (black line) along with the corrected LFs. The correction for Malmquist bias (blue line) and Malmquist bias with binaries (red line) is quite similar at high luminosities (indicating binaries do not strongly influence the $\mathrm{LF}$ ), but at low luminosities, the binary correction becomes more important.

\subsection{Results: Galactic Structure}

The resulting Galactic structure parameters are discussed and compared to previous results in this section. While this was not the main focus of the investigation, it represents one of the largest star-count samples ever employed to measure the stellar 


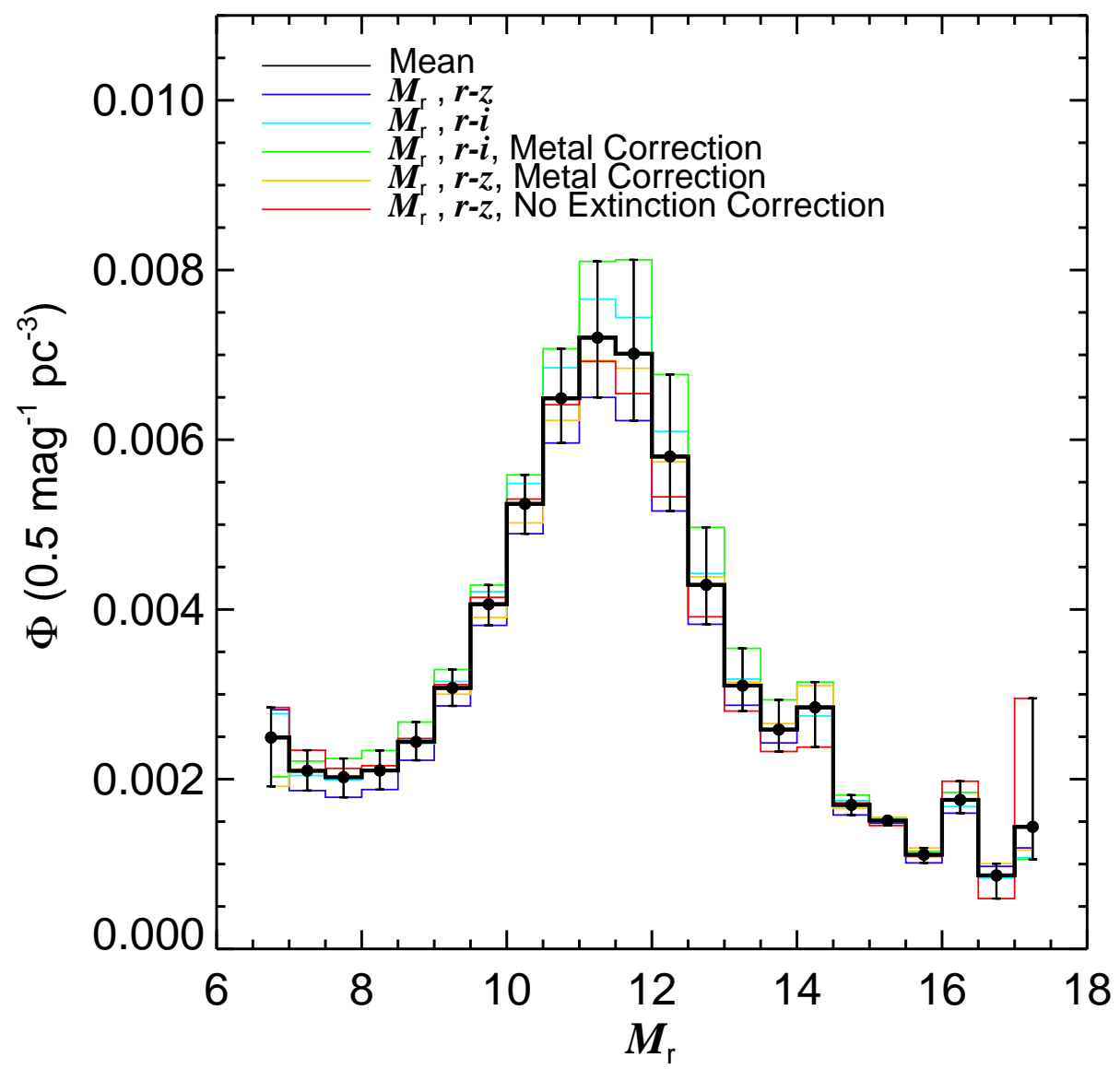

Figure 5.20 Shown is the average, bias-corrected LF, along with individual LFs measured with five different CMRs. The colors corresponding to each CMR are given in the legend. Since the CMR is the dominant systematic in this analysis, error bars were chosen to reflect the entire observed spread in each LF bin. 
Table 5.6. Effects of Systematic CMR changes on Galactic Structure Results

\begin{tabular}{rllllll}
\hline \hline Model & $Z_{\mathrm{o}, \text { thin }}(\mathrm{pc})$ & $R_{\mathrm{o}, \text { thin }}(\mathrm{pc})$ & $Z_{\mathrm{o}, \text { thick }}(\mathrm{pc})$ & $R_{\mathrm{o}, \text { thick }}(\mathrm{pc})$ & $f$ & $\chi^{2}$ \\
\hline$\left(M_{r}, r-z\right)$ & 260 & 2260 & 1270 & 4340 & 0.96 & 3.22 \\
$\left(M_{r}, r-i\right)$ & 244 & 2130 & 1112 & 4280 & 0.96 & 3.34 \\
$\left(M_{r}, r-z\right)+0.5$ & 220 & 2020 & 1050 & 3100 & 0.97 & 3.88 \\
$\left(M_{r}, r-z\right)+0.5$ & 215 & 2010 & 920 & 3000 & $0.96^{\mathrm{a}}$ & 3.87 \\
$\left(M_{r}, r-z\right)-0.5$ & 280 & 2180 & 860 & 5930 & 0.90 & 2.64 \\
$\left(M_{r}, r-z\right)-0.5$ & 310 & 2490 & 1500 & 7770 & $0.96^{\mathrm{a}}$ & 2.65 \\
$\left(M_{r}, r-z\right)$, no extinction & 250 & 2220 & 1410 & 4940 & 0.97 & 3.75 \\
{$[F e / H](Z)$ Gradient } & 270 & 2250 & 1700 & 3460 & 0.97 & 3.52 \\
{$[F e / H](Z)$ Gradient } & 260 & 2220 & 1210 & 3320 & $0.96^{\mathrm{a}}$ & 3.52 \\
\hline
\end{tabular}

${ }^{a}$ Galactic Structure parameters measured while holding the thin disk fraction fixed at 0.96 .

density of the Milky Way's Disk. This analysis is quite similar to Jurić et al. (2008), which used a larger set of SDSS photometry and a slightly different CMR to measure stellar densities. Their sample contained $\sim 48$ million stellar observations and mapped stellar densities out to distances of $10 \mathrm{kpc}$. Their main goal was to measure Galactic structure, not the LF. Their color bins are not evenly spaced in absolute magnitude, complicating direct comparison of LFs (see §5.7.1). Although my thick disk scale height and normalization are somewhat different, the thin disk values, which are my best measured parameters, are in good agreement, as shown in Table 5.7. This is encouraging, since these two investigations used different (but similar) photometric parallax relations (see Figure 4.1) and density calculation methods. It suggests that the wide areal angle and precision photometry of SDSS is a robust tool for examining the stellar structure of the Milky Way. Further investigations and improvements in the CMR (Golimowski et al., 2009; Sesar et al., 2008) will improve the accuracy of these studies.

The thin disk fraction measured in this study is among the larger values estimated 
Table 5.7. Galactic Structure Parameters

\begin{tabular}{rllllllllll}
\hline \hline \multicolumn{1}{c}{ Study } & $Z_{\mathrm{o}, \text { thin }}(\mathrm{pc})$ & \multicolumn{2}{l}{$R_{\mathrm{o}, \text { thin }}(\mathrm{pc})$} & \multicolumn{2}{l}{$Z_{\mathrm{o}, \text { thick }}(\mathrm{pc})$} & $R_{\mathrm{o}, \text { thick }}(\mathrm{pc})$ & \multicolumn{2}{c}{$f$} \\
\hline & Obs. & Corr. & Obs. & Corr. & Obs. & Corr. & Obs. & Corr. & Obs. & Corr. \\
Jurić et al. (2008) & 245 & 300 & 2150 & 2600 & 743 & 900 & 3261 & 3600 & 0.87 & 0.87 \\
This Study & 255 & 320 & 2200 & 2750 & 1360 & 1400 & 4100 & 4500 & 0.97 & 0.98 \\
\hline
\end{tabular}

with star counts (see Table 1 and Figure 1 of Siegel et al., 2002). Kinematic studies, such as Reid et al. (2002), suggest a smaller thin disk fraction, near 90\%, which is similar to the results of Jurić et al. (2008). As discussed above, the dispersion in the observed values of the normalization of the thin disk is enhanced by the correlation between the thick disk fraction and the assumed scale heights. However, the normalization does not strongly affect the observed LF, as shown in Figure 5.16.

\subsection{Results: Luminosity Function}

Finally, the luminosity function is constructed from the local densities fit to each absolute magnitude slice. The raw LF, prior to being corrected for the biases discussed above, was shown in Figure 5.12. The final LF is presented in Figure 5.20. The final LF rises smoothly to a peak near $M_{r}=11$, corresponding to a spectral type $\sim \mathrm{M} 3$, followed by a decline at lower luminosities. Below, this final $M_{r}$ LF is compared to previous studies. The $M_{r} \mathrm{LF}$ is then transformed to the $J$ band, and compared to previous studies in that filter. Particularly, the $M_{J} \mathrm{LF}$ is compared to the photometric and spectroscopic survey of Covey et al. (2008) and the local, volume-complete trig parallax sample of Reid \& Gizis (1997).

\subsubsection{Comparison to previous results: $M_{r}$}

Many previous studies of the low-mass field LF have used the $V$ or $J$ filters. This study is the first to measure the $M_{r}$ LF, but Hawkins \& Bessell (1988) published a Schmidt plate $R$ band LF of M dwarfs. Their sample was drawn from 431 stars 
Table 5.8. Final $M_{r}$ Luminosity Function

\begin{tabular}{llll}
\hline \hline$M_{r}$ bin & $\Phi_{\text {Mean }}$ & $\Phi_{\text {Max }}$ & $\Phi_{\text {Min }}$ \\
\hline 6.75 & 2.491 & 2.845 & 1.915 \\
7.25 & 2.099 & 2.341 & 1.866 \\
7.75 & 2.024 & 2.244 & 1.787 \\
8.25 & 2.103 & 2.338 & 1.878 \\
8.75 & 2.440 & 2.673 & 2.223 \\
9.25 & 3.075 & 3.292 & 2.863 \\
9.75 & 4.061 & 4.288 & 3.813 \\
10.25 & 5.244 & 5.587 & 4.893 \\
10.75 & 6.488 & 7.073 & 5.961 \\
11.25 & 7.203 & 8.102 & 6.499 \\
11.75 & 7.013 & 8.120 & 6.225 \\
12.25 & 5.802 & 6.769 & 5.161 \\
12.75 & 4.290 & 4.968 & 3.825 \\
13.25 & 3.103 & 3.541 & 2.803 \\
13.75 & 2.585 & 2.935 & 2.326 \\
14.25 & 2.846 & 3.144 & 2.378 \\
14.75 & 1.697 & 1.812 & 1.578 \\
15.25 & 1.512 & 1.550 & 1.454 \\
15.75 & 1.110 & 1.189 & 1.014 \\
16.25 & 1.756 & 1.976 & 1.600 \\
16.75 & 0.866 & 1.005 & 0.593 \\
17.25 & 1.439 & 2.953 & 1.055 \\
\hline & & &
\end{tabular}

Note. - Densities are reported in units of (stars $\mathrm{pc}^{-3} 0.5 \mathrm{mag}^{-1}$ ) $\times 10^{-3}$. 
located within 100 pc. Due to the small sample size, the statistical uncertainties are quite large. However, since the $R$ filter is quite similar to the $r$ band, a comparison of LFs is warranted. Shown in Figure 5.21 is the LF from Hawkins \& Bessell (1988) compared to the LF from this study. While the overall scaling is in disagreement, the general shapes of the two LFs are in accord, both peaking near $M_{r}=11$. The likely cause of the disagreement in the scaling is probably a systematic underestimate of distance, which would increase the measured density.

The Jurić et al. (2008) study uses color bins spaced every 0.1 mags in $r-i$, though not evenly spaced in absolute magnitude, their densities can be interpolated onto my grid. Shown in Figure 5.22 is my $M_{r}$ LF of compared to the "joint fit, bright parallax" results of Jurić et al. (2008), assuming 10\% error bars. The two LFs agree, although their work only probes to $M_{r} \sim 11$, due to their red limit of $r-i \sim 1.4$. The differences between our LFs can be attributed to the different CMRs, since their CMR predicts smaller distances for $M_{r}<9.5$ and larger distance for $M_{r}>9.5$ (see Figure 4.5). These shifts correspond to an increase and decrease in the local density, respectively.

\subsection{2 $M_{J} L F$}

Luminosity functions can be transformed between filter bandpasses using Equation 4.2. This transformation is straightforward, provided the derivative $d M_{\lambda, 1} / d M_{\lambda, 2}$ is known. In $\S 4.4 .3, M_{J}\left(M_{r}\right)$ (Equation 4.3), was empirically determined by fitting the absolute magnitude locus for nearby stars. Using the derivative of this relation, the $M_{r} \mathrm{LF}$ is transformed into an $M_{J} \mathrm{LF}$ (see Equation 4.2). The resulting $J$ band LF is shown in Figure 5.23 and given in Table 5.9.

The J filter has traditionally been used as a tracer of mass (Delfosse et al., 2000) and bolometric luminosity (Golimowski et al., 2004) in low-mass stars, since it samples the SED near its peak. The largest field LF investigation to date, Covey et al. (2008), determined the $J$ band LF from $M_{J}=4$ to $M_{J}=12$. Below, the present 


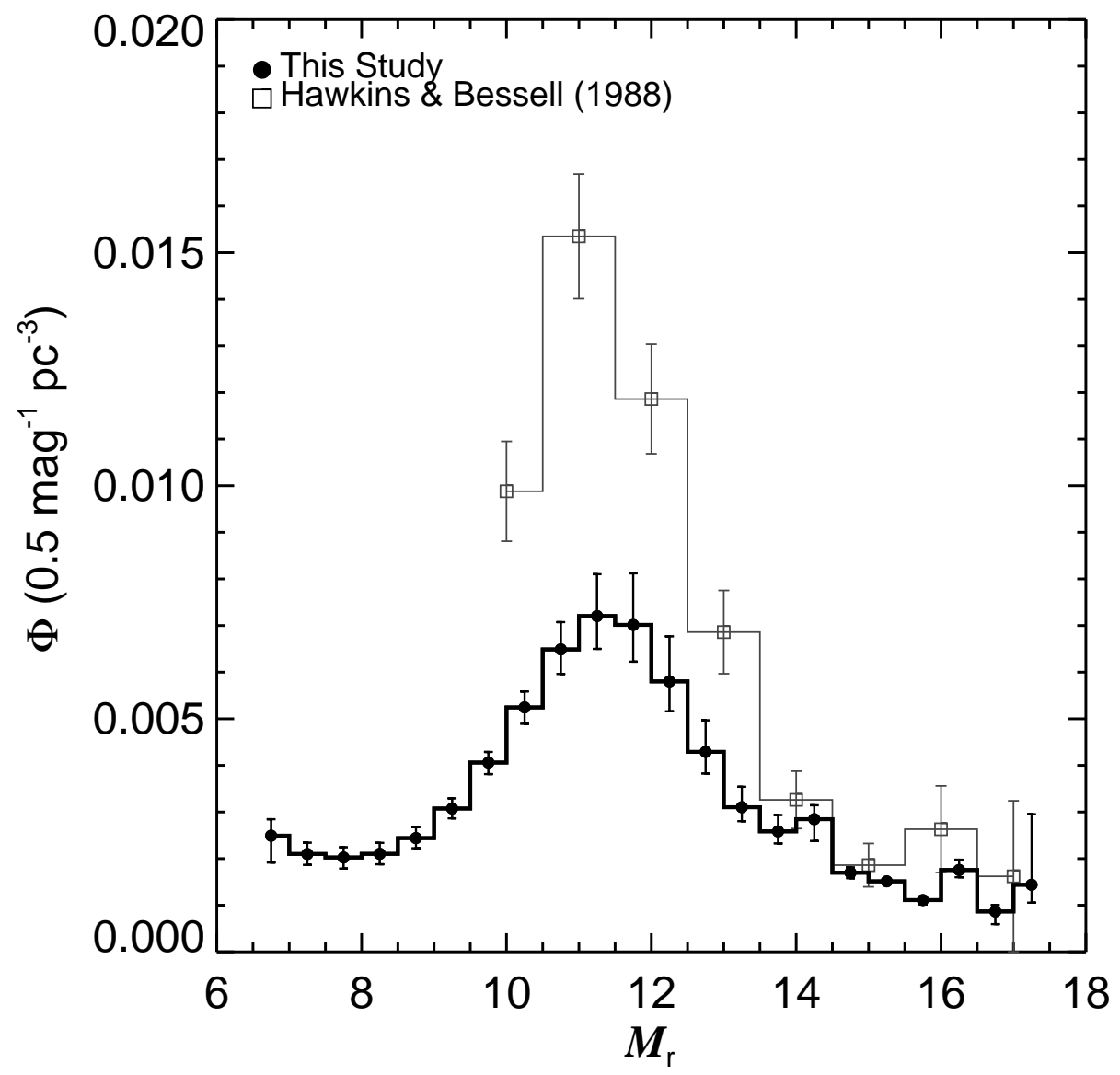

Figure 5.21 Shown is the $M_{r}$ LF (filled circles) compared to the LF measured by Hawkins \& Bessell (1988) (open squares). Note the agreement in the location of the peak of each LF. 


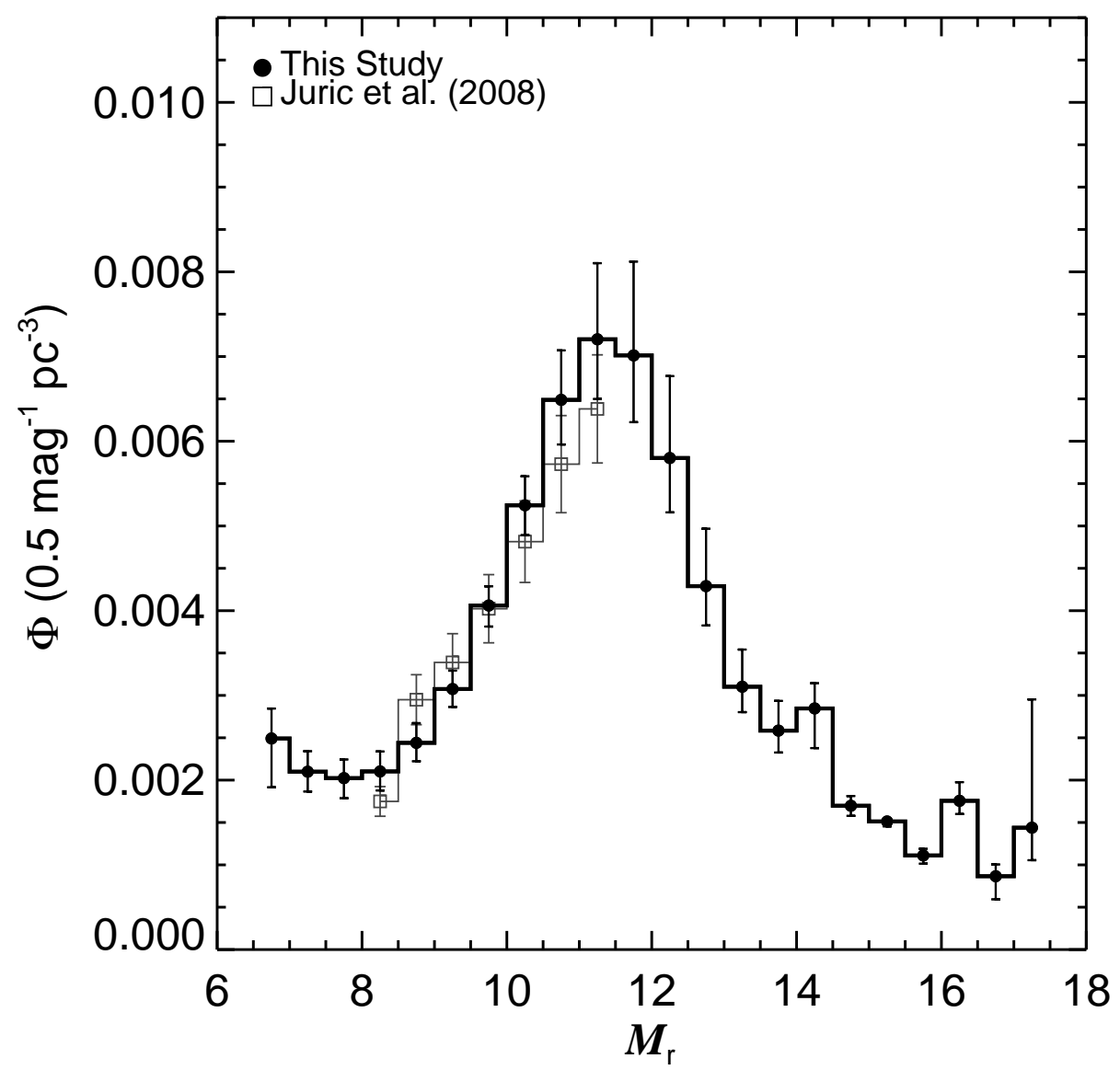

Figure 5.22 Shown is the $M_{r}$ LF (filled circles) compared to the LF measured by Juric et al. (2008) (open squares). Their sample did not probe fainter absolute magnitudes, and employed a different CMR, which accounts for the offsets between the LFs. 
study is compared to the Covey et al. (2008) investigation.

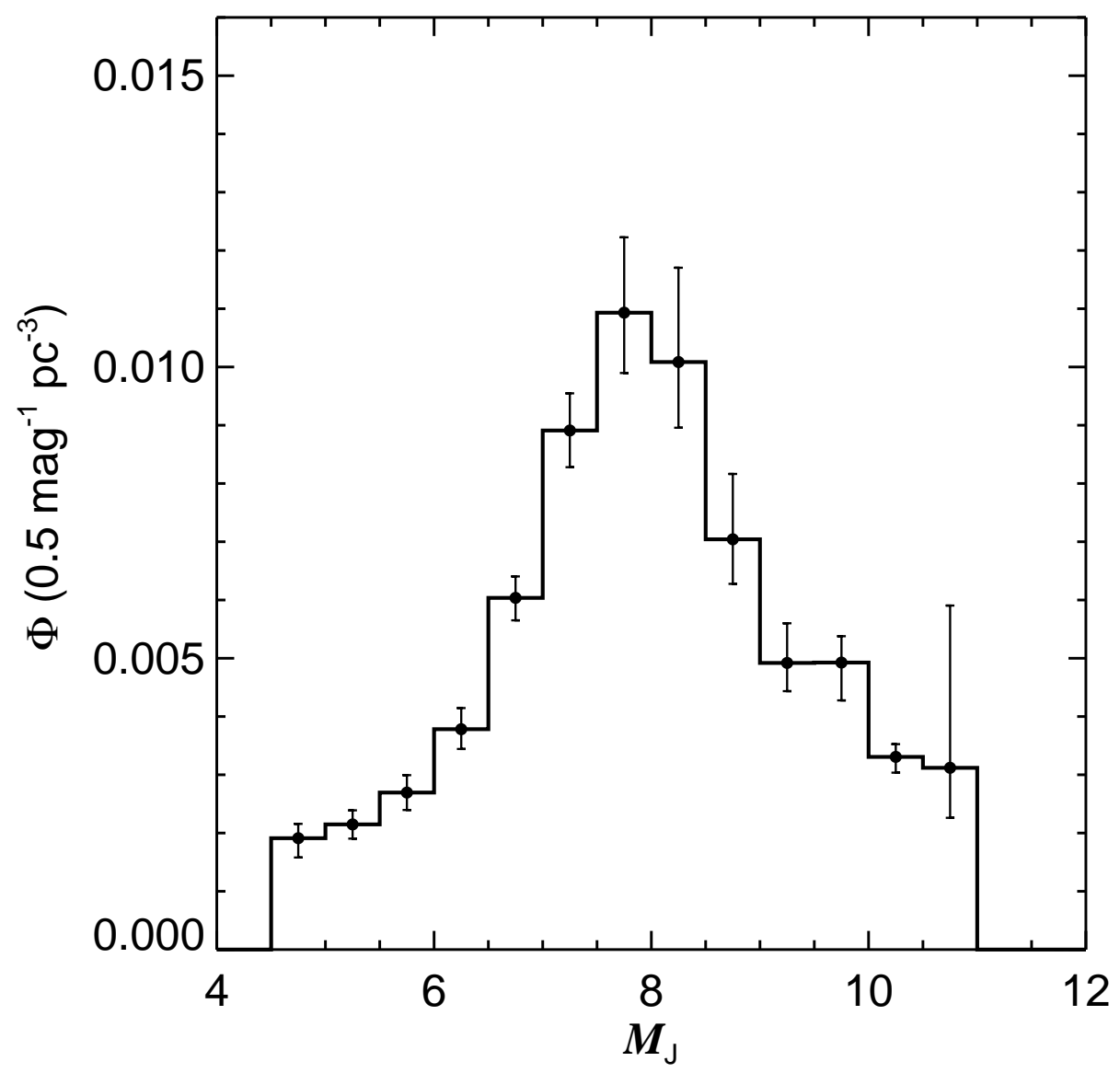

Figure 5.23 Shown is the $J$ band LF for this sample. Note the peak near $M_{J}=8$ and the monotonic decline at lower magnitudes.

Comparison to Covey et. al

In Figure 5.24, the transformed $M_{J} \mathrm{LF}$ is plotted with the $M_{J}$ LF from Covey et al. (2008). These two LFs agree quite well, both peaking near $M_{J}=8$. There is a slight systematic offset at high luminosities, with the $M_{J}$ LF being consistently higher than the Covey et al. (2008) study. This is most likely due to the different CMRs employed by the two studies. Covey et al. (2008) uses an $\left(M_{i}, i-J\right)$ CMR, as opposed to the $\left(M_{r}, r-z\right)$ CMR employed in the current study. The different filters and CMRs could 
Table 5.9. $\quad M_{J}$ Luminosity Function

\begin{tabular}{llll}
\hline \hline$M_{J}$ bin & $\Phi_{\text {Mean }}$ & $\Phi_{\text {Max }}$ & $\Phi_{\text {Min }}$ \\
\hline 4.75 & 1.911 & 2.158 & 1.580 \\
5.25 & 2.148 & 2.390 & 1.904 \\
5.75 & 2.695 & 2.993 & 2.395 \\
6.25 & 3.782 & 4.145 & 3.443 \\
6.75 & 6.036 & 6.405 & 5.651 \\
7.25 & 8.910 & 9.548 & 8.280 \\
7.75 & 10.932 & 12.226 & 9.897 \\
8.25 & 10.085 & 11.703 & 8.957 \\
8.75 & 7.042 & 8.164 & 6.277 \\
9.25 & 4.920 & 5.601 & 4.436 \\
9.75 & 4.926 & 5.378 & 4.277 \\
10.25 & 3.307 & 3.526 & 3.036 \\
10.75 & 3.120 & 5.904 & 2.261 \\
\hline
\end{tabular}

Note. - Densities are reported in units of $\left(\right.$ stars pc $\left.^{-3} 0.5 \mathrm{mag}^{-1}\right) \times 10^{-3}$.

easily produce differences of this magnitude.

Since the LF measured by Covey et al. (2008) was derived from SDSS observations, their results provide a natural test of the new method for measuring the LF. Covey et al. (2008) employed the traditional $V_{\text {eff }}$ formalism (Schmidt, 1968; Felten, 1976), summing up the volume probed by each star and normalizing by the Galaxy's underlying Galactic structure. This was appropriate, since their sample was along one line of sight. As stated above, my sample is spread over $1 / 4$ of the sky, making the traditional $V_{\text {eff }}$ calculation very computationally intensive. ${ }^{11}$ Thus, a direct $V_{\text {eff }}$ computation for my sample is not feasible. Yet, the excellent agreement between the two studies indicates that volumes and derived densities were properly calculated for the present investigation. This agreement serves as an empirical verification of the

\footnotetext{
${ }^{11}$ In order to numerically integrate the effective volume for each of the 15 million stars in our sample, approximately 50 billion mathematical operations are required, assuming one does not fit for Galactic structure.
} 
new LF technique presented in this dissertation.

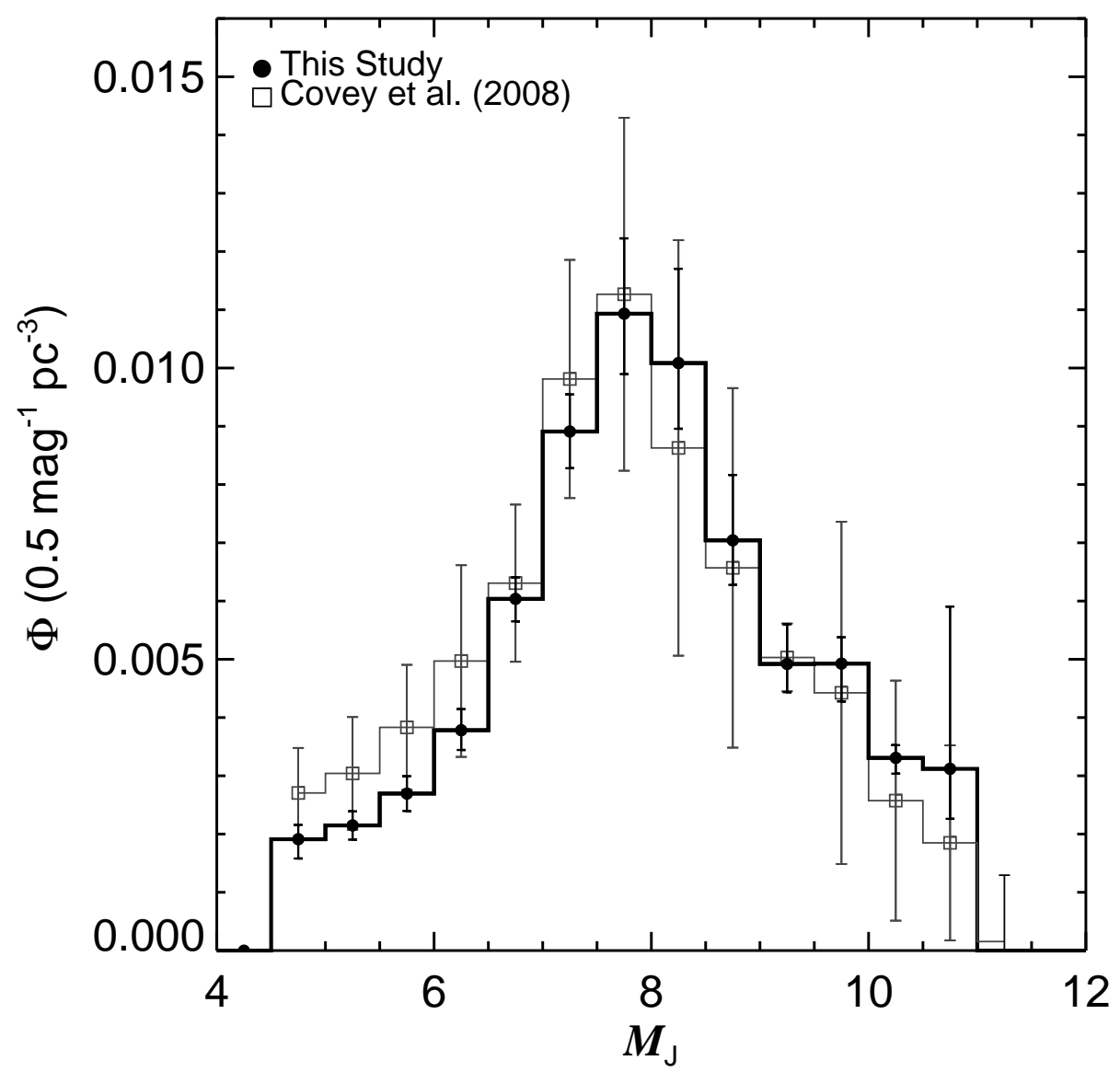

Figure 5.24 Shown is the $M_{J}$ LF compared to the LF measured by Covey et al. (2008). Note the excellent agreement in shape and scaling, ensuring that the densities estimated by this study are correct.

\section{Comparison to Local LFs}

Finally, a comparison to luminosity functions from nearby, trig parallax stars (Reid \& Gizis, 1997) is appropriate. As previously discussed, nearby samples benefit from accurate distances, avoiding the pitfalls associated with the photometric parallax technique. Moreover, nearby binary stars are easily resolved, either astrometrically or with radial velocity measurements. Unfortunately, local surveys are limited in size 
and suffer from Poisson errors larger than those derived from photometric surveys.

In Figure 5.25, the $M_{J}$ LF is compared to the LF of primaries and secondaries first measured by Reid \& Gizis (1997) and updated by Cruz et al. (2007). These stars are drawn from a volume-complete sample with $d<8$ pc. A total of 146 stars in 113 systems are found within these limits. Again, there is broad agreement between the $M_{J} \mathrm{LF}$ and the volume-complete sample. Note the error bars on the $M_{J} \mathrm{LF}$ are much smaller than many in the Reid \& Gizis (1997) LF, even with conservative systematic error measurements. While the Reid \& Gizis (1997) LF error budget is dominated by Poisson statistics, these are minimized in the current sample (many absolute magnitude bins contain over one million stars). The differences seen between the two samples can be attributed to the assumed CMR and the small sample size of the Reid \& Gizis (1997) study. The form of this relation imposes strong systematics on the resulting LF. ${ }^{12}$ Yet, the broad agreement between the local and photometric LFs is promising, indicating that the photometric and volume-complete methods now give similar results.

\subsection{Conclusions}

A new $M_{r}$ luminosity function for field low-mass stars in SDSS is presented. This analysis used $\sim 15$ million stars, making it three orders of magnitude larger than previous studies. Due to its wide areal coverage, the traditional $V_{\text {eff }}$ formalism would be computationally untenable. Thus, a method similar to the studies of Juric et al. (2008) and Gould et al. (1996) is introduced to measure the luminosity function. This technique computes the $(R, Z)$ stellar density distribution for small (0.5 mag) slices in absolute magnitude. A Galactic model is then fit to the resulting map and the local density is recorded as a function of $M_{r}$. This method simultaneously determines Galactic structure parameters and the LF, usually impossible for single pencil beam

\footnotetext{
${ }^{12}$ This is not surprising, as how absolute magnitudes are assigned should significantly affect the local absolute magnitude distribution.
} 


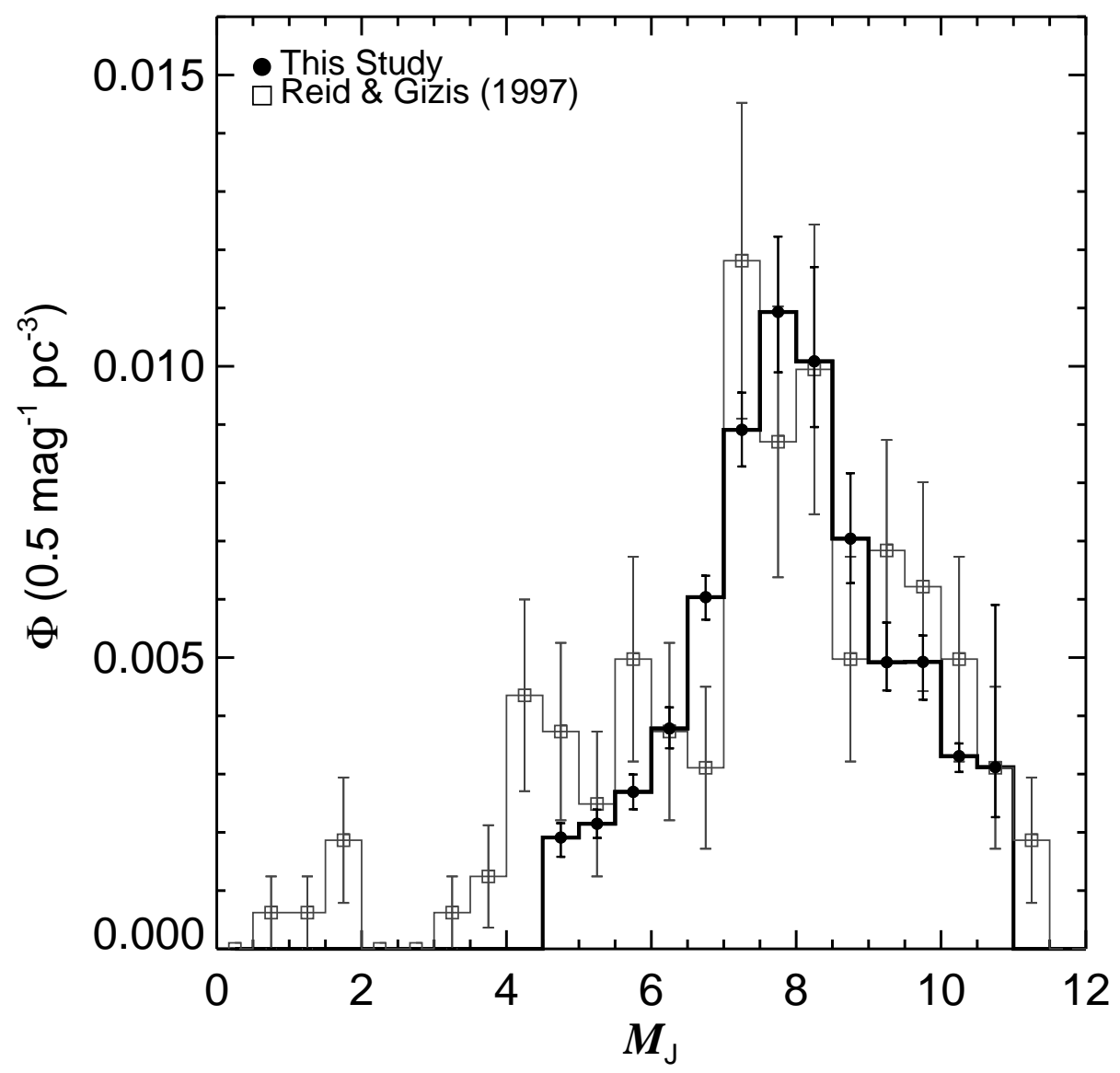

Figure 5.25 Shown is the $M_{J}$ LF (filled circles) compared to the LF measured by (Reid et al., 2002) (open squares). The two LFs agree, resolving previous discrepancies between the photometric and volume-complete samples. 
surveys or local, volume-complete samples.

The derived Galactic structure parameters are compared to previous studies, finding broad agreement with modern surveys. In particular, this study agrees well with the work of Jurić et al. (2008). This may not be surprising, since both studies use similar CMRs to derive stellar densities from SDSS observations. However, the Jurić et al. (2008) study does not report a LF, and uses color bins, rather than absolute magnitude bins, to study the stellar density distributions.

Understanding the errors and bias imposed by the observations and analysis is crucial for accurately determining the LF. An extensive suite of Monte-Carlo simulations were constructed to determine the individual and combined effects of: Malmquist bias, unresolved binarity, and the fitting routine. The impact of these biases on both Galactic structure and the observed LF are discussed. Systematic errors in the CMR, either innate or due to metallicity, produce the largest relative changes in the final LF. The best fit Galactic structure parameters are reported in Table 5.5 and the $M_{r}$ LF is listed in Table 5.8.

Many existing field LFs have been measured in $V$ or $J$. The $M_{r}$ LF is transformed into $M_{J}$, using the absolute magnitude transformation from Chapter 4 . This $M_{J} \mathrm{LF}$ is compared directly to the studies of Covey et al. (2008) and Reid \& Gizis (1997). The Covey et al. (2008) sample is drawn from SDSS / 2MASS photometry along one line of sight, and their LF is measured using the $V_{\text {eff }}$ formalism. Excellent agreement is found between the two LFs, indicating the method used in the present study to compute densities is sound. This serves as an empirical, rather than formal, proof of concept.

The assumed CMR is tested using the volume-complete, trig parallax sample of Reid \& Gizis (1997). This study surveyed the nearest 8 pc, deriving the $M_{J}$ band LF from $\sim 150$ stars. The agreement between the two studies shows that the volumecomplete and photometric methods now give similar results, but the photometric technique has much greater precision. The trigonometric parallaxes obviate many 
of the issues faced by photometric parallaxes, but suffers from significant Poisson errors. Future trig parallax surveys, such as GAIA and LSST, will remedy this situation. These missions will observe thousands of stars, extending the distance limits of volume-complete trig parallax samples to 100s of parsecs, significantly reducing the Poisson noise in nearby samples. 


\section{Chapter 6}

\section{MASS FUNCTION OF FIELD LOW-MASS DWARFS}

\subsection{Introduction}

The mass function (MF), $\Psi(\mathrm{M})$, or the number of stars per unit mass per unit volume, is a fundamental quantity that influences a wide range of astronomical topics. It succinctly describes the star formation process, specifying how molecular clouds redistribute their mass into stars. Mass-to-light ratios are constructed from the MF and used to measure the stellar mass of external galaxies (e.g., Bell \& de Jong, 2001). Furthermore, the MF is employed to model the chemical and kinematic evolution of the Galaxy (e.g., Romano et al., 2005). Thus, an accurate measurement of the MF is central to both star formation theory and Galactic structure and evolution.

The mass of a star is not directly observed (except for eclipsing binaries), so other properties, such as the bolometric luminosity or absolute magnitude are used as proxies. This is accomplished through mass-luminosity relations (MLRs) derived from models or eclipsing binary observations (e.g., Henry \& McCarthy, 1993; Kroupa \& Tout, 1997; Delfosse et al., 2000) ${ }^{1}$. Once the shape of the MLR is known, transforming the observed LF into a MF is relatively straightforward:

$$
\Psi(\mathrm{M})=\frac{d N}{d \mathrm{M}}=\frac{d N}{d M}\left|\frac{d M}{d \mathrm{M}}\right|=\Phi(M)\left|\frac{d M}{d \mathrm{M}}\right|
$$

where $d M / d \mathrm{M}$ is the derivative of the MLR. The application of the previous equation would be trivial if mass depended solely upon luminosity. Unfortunately, age and

\footnotetext{
${ }^{1}$ While they are commonly known as mass-luminosity relations, most empirical MLRs are actually mass-absolute magnitude relations.
} 
metallicity can also affect a star's luminosity. Thus, care must be taken in order to account for these effects.

Since M dwarfs have main sequence lifetimes greater than the Hubble time (Laughlin et al., 1997), they undergo essentially no luminosity evolution over the lifetime of the Galaxy. Simply put, every field M dwarf ever created is still on the main sequence today $^{2}$. This avoids the age problem, and presents the opportunity to explore another fundamental quantity, the initial mass function (IMF, Salpeter, 1955; Miller \& Scalo, 1979; Scalo, 1986). The IMF is derived from the observed present-day mass function (PDMF) with assumptions about the stellar birthrate and stellar evolution. For $\mathrm{M}$ dwarfs, assuming the low-mass IMF has remained constant over the lifetime of the Milky Way, the PDMF is the IMF (Miller \& Scalo, 1979).

Following Salpeter (1955), the IMF has traditionally been characterized by a power law:

$$
\Psi(\mathrm{M})=\frac{d N}{d \mathrm{M}} \propto \mathrm{M}^{-\alpha}
$$

where $\alpha=2.35$ is known as the Salpeter slope. Since stellar masses can range from $\sim 100 \mathrm{M}_{\odot}$ to $0.08 \mathrm{M}_{\odot}$, it is often convenient to measure the IMF in units of log mass:

$$
\xi(\log \mathrm{M})=\frac{d N}{d(\log \mathrm{M})} \propto \mathrm{M}^{\Gamma}
$$

where $\Gamma=1-\alpha$. In these units, the Salpeter slope is $\Gamma=-1.35$. Power laws with $\alpha>2.35(\Gamma<-1.35)$ are "steeper" than the Salpeter slope, and "flatter" IMFs have an $\alpha<2.35(\Gamma>-1.35)$. Steeper slopes predict a higher ratio of low-mass to high-mass stars compared to their flatter counterparts. Many studies (Tinney, 1993; Kroupa, 2002; Covey et al., 2008) have found that a single power law is insufficient to describe the IMF in the low-mass regime $\left(0.1 \mathrm{M}_{\odot}<\mathrm{M}<0.8 \mathrm{M}_{\odot}\right)$. Thus, multiple "broken" power laws are fit to the MF, resulting in multiple values of $\alpha$. Other

\footnotetext{
${ }^{2}$ This is not the case in star-forming regions, where low-mass protostars are still contracting towards the main sequence.
} 
mathematical forms have also been used to describe the IMF, most notably the lognormal distribution (e.g., Miller \& Scalo, 1979; Chabrier, 2003a):

$$
\xi(\log \mathrm{M})=C_{\circ} \exp \left(\frac{-\left(\log \mathrm{M}-\log \left(\mathrm{M}_{\circ}\right)\right)^{2}}{2 \sigma^{2}}\right)
$$

where $C_{\circ}$ is the overall density scaling, $\mathrm{M}_{\circ}$ is the characteristic mass, and $\sigma$ is the width of the distribution. The log-normal distribution smoothly mimics the effects of multiple power laws. I stress that these mathematical formalisms do not carry any physical significance. They are merely convenient parameterizations of the data. However, theoretical investigations of the IMF have shown that a log-normal distribution is readily obtained from sampling a range of initial physical parameters (Elmegreen \& Mathieu, 1983), which may simply be a manifestation of the central limit theorem ${ }^{3}$.

Observational studies of the low-mass IMF have focused on two sources: clusters and the field. Each has its strengths and weaknesses. Open clusters and star forming regions form co-eval populations at a single distance, simplifying luminosity determinations. Yet, the number of stars in a given cluster is limited and pre-main sequence objects constitute a significant fraction of younger clusters and star-formation regions, complicating the analysis. Furthermore, proper-motions are needed to separate the foreground field stars from the cluster members. Finally, clusters undergo dynamic segregation, with the least-massive stars are ejected and no longer bound due to gravitational interactions ("collisions") with higher mass cluster members (e.g., Fan et al., 1996). Thus, an unbiased determination of a cluster's IMF requires extensive imaging from the core to the edge of the association (and may still miss cluster members due to crowding).

On the other hand, the field is dominated by main sequence stars, but as shown in

\footnotetext{
${ }^{3}$ The central limit theorem states that the mean of large number of samples from the same underlying distribution will be Gaussian, even if the parent distribution is not.
} 
Chapter 4, measuring their intrinsic luminosity is difficult. Moreover, field observations are usually limited in either depth or area, since observing a wide area on the sky to faint brightness limits requires a prohibitive amount of time ${ }^{4}$. Thus, uncertainty currently exists in the exact form of the low-mass IMF. Table 6.1 list the properties of major modern field mass function surveys, along with my own. Using the largest photometric dataset of field $\mathrm{M}$ dwarfs ever assembled, I aim to remedy this situation with a new measurement of the field low-mass IMF.

This chapter details my study of the low-mass stellar IMF. In $\S 6.2$, the transformation of the observed LF (see Chapter 5) into a MF is described. The results are shown in $\S 6.3$, along with a comparison to previous field IMF measurements. The conclusions follow in $\S 6.4$.

\subsection{Analysis}

\subsubsection{Mass-Luminosity Relations}

The assumed form of the MLR dictates the transformation from luminosity to mass. Shown in Figure 6.1 are contemporary MLRs for low-mass stars in the V and J bands (Henry \& McCarthy, 1993; Kroupa et al., 1993; Baraffe et al., 1998; Delfosse et al., 2000; Xia et al., 2008) along with stars with empirically determined masses and $J$ and $V$ band absolute magnitudes (from Delfosse et al., 2000). Note that at infrared wavelengths, the scatter among MLRs is reduced compared to the optical. Metallicity was first suggested as the cause of the increased dispersion by Delfosse et al. (2000), and was confirmed by Bonfils et al. (2005). At high metallicities, increased line-blanketing redistributes flux to longer wavelengths. However, stars with higher metallicities will have smaller bolometric luminosities at the same mass. These two effects counteract each other at near-IR wavelengths, decreasing the effect of metallicity on the MLR. Thus, the $M_{J}$-Mass relation of Delfosse et al. (2000) is adopted as the fiducial MLR.

\footnotetext{
${ }^{4}$ Where prohibitive is defined as $\gg$ the average lifetime of a graduate student (currently six
} 
Table 6.1. Major Low-Mass Field IMF Studies

\begin{tabular}{|c|c|c|c|c|c|c|c|}
\hline Authors & $N_{\text {Stars }}$ & $\Omega$ (sq. deg.) & Filter(s) & Depth & Mass Range & $\alpha, \mathrm{M}_{\circ}$ & Notes \\
\hline Salpeter (1955) & $\cdots$ & $\cdots$ & $V$ & $\cdots$ & $0.3 \mathrm{M}_{\odot}-10 \mathrm{M}_{\odot}$ & 2.35 & Compiled LFs from contemporaries ${ }^{\mathrm{a}}$ \\
\hline Miller \& Scalo (1979) & $\ldots$ & $\ldots$ & $V$ & $\ldots$ & $0.1 \mathrm{M}_{\odot}-60 \mathrm{M}_{\odot}$ & $\mathrm{M}_{\circ} \simeq 0.1 \mathrm{M}_{\odot}$ & Log-normal fit, Compilation of 3 field LFs ${ }^{\mathrm{b}}$ \\
\hline Kroupa et al. (1990) & $\cdots$ & $\cdots$ & $V$ & $\cdots$ & $0.1 \mathrm{M}_{\odot}-0.9 \mathrm{M}_{\odot}$ & $\mathrm{M}_{\circ} \simeq 0.23 \mathrm{M}_{\odot}, \alpha=0.70$ & Adopted LFs of Scalo (1986) and Stobie et al. (1989) \\
\hline Kroupa et al. (1993) & $\cdots$ & $\cdots$ & $V, I$ & $\cdots$ & $0.08 \mathrm{M}_{\odot}-0.5 \mathrm{M}_{\odot}$ & $0.70<\alpha<1.85$ & LF from Wielen et al. (1983) and Stobie et al. (1989) \\
\hline Tinney (1993) & 3,500 & $280 \operatorname{deg}^{2}$ & $I, K$ & $I \lesssim 17.5$ & $0.1 \mathrm{M}_{\odot}-0.5 \mathrm{M}_{\odot}$ & $\ldots$ & Turnover at $0.25 \mathrm{M}_{\odot}$ \\
\hline Reid \& Gizis (1997) & 151 & $\delta>-30^{\circ}$ & $V, I$ & $d \tilde{<} 8 \mathrm{pc}$ & $0.08 \mathrm{M}_{\odot}-1.2 \mathrm{M}_{\odot}$ & 1.2 & Solar Neighborhood ${ }^{\mathrm{c}}$ \\
\hline Martini \& Osmer (1998) & 1,500 & $0.83 \mathrm{deg}^{2}$ & $V, R$ & $V \lesssim 23.5$ & $0.1 \mathrm{M}_{\odot}-0.6 \mathrm{M}_{\odot}$ & 1.3 & \\
\hline Zheng et al. (2001) & $\sim 1,400$ & $\sim 0.4 \mathrm{deg}^{2}$ & $V, I$ & $18 \lesssim I \lesssim 24$ & $0.1 \mathrm{M}_{\odot}-0.6 \mathrm{M}_{\odot}$ & 1.45 & HST observations \\
\hline Kroupa (2002) & $\ldots$ & $\ldots$ & $V, I$ & $\ldots \sim \sim$ & $0.08 \mathrm{M}_{\odot}-0.50 \mathrm{M}_{\odot}$ & 1.3 & Compiled contemporary LFs ${ }^{\mathrm{d}}$ \\
\hline Reid et al. (2002) & 558 & $3 \pi$ ster. & $B, V$ & $d \lesssim 20 \mathrm{pc}$ & $0.1 \mathrm{M}_{\odot}-3.0 \mathrm{M}_{\odot}$ & $\sim 1.3$ & Solar Neighborhood Survey \\
\hline Chabrier (2003a) & $\cdots$ & $\ldots$ & $V, K$ & $\ldots$ & $0.1 \mathrm{M}_{\odot}-1.0 \mathrm{M}_{\odot}$ & $\mathrm{M}_{\circ}=0.22 \mathrm{M}_{\odot}$ & Review of contemporary field LFs $\mathrm{e}$ \\
\hline Schultheis et al. (2006) & 3,600 & $\sim 3 \operatorname{deg}^{2}$ & $r^{\prime}, i^{\prime}$ & $i^{\prime} \sim 21$ & $\mathrm{M}<0.25 \mathrm{M}_{\odot}$ & 2.5 & CFHT MegaCAM observations \\
\hline Covey et al. (2008) & 29,000 & $30 \mathrm{deg}^{2}$ & $i, J$ & $J=16.2$ & $0.1 \mathrm{M}_{\odot}-0.8 \mathrm{M}_{\odot}$ & $\mathrm{M}_{\circ}=0.29 \mathrm{M}_{\odot}$ & Matched SDSS \& 2MASS observations \\
\hline This Study & $\sim 15,000,000$ & $8,400 \mathrm{deg}^{2}$ & $r, i, z$ & $16<r<22$ & $0.1 \mathrm{M}_{\odot}-0.8 \mathrm{M}_{\odot}$ & $\mathrm{M}_{\circ}=0.27 \mathrm{M}_{\odot}$ & SDSS observations \\
\hline
\end{tabular}

${ }^{a}$ Salpeter averaged luminosity functions from van Rhijn $(1925,1936)$ and Luyten $(1939,1941)$.

${ }^{\mathrm{b}}$ Their adopted LF was averaged from the LFs of McCuskey (1966), Luyten (1968) and Wielen (1974).

"The "8 parsec" sample was compiled by Reid \& Gizis (1997), with later additions from Reid et al. (1999), Reid et al. (2003b), and Cruz et al. (2007).

${ }^{\mathrm{d}}$ Kroupa (2002) presents a comprehensive summary of MFs derived from the field and clusters over a wide mass range. For low-mass stars in the field, he refers to Reid et al. (1999), Herbst et al. (1999), Chabrier (2001) and Zheng et al. (2001).

e Chabrier (2003a) compared the LFs of Dahn et al. (1986), Henry \& McCarthy (1990) and Zheng et al. (2001). 

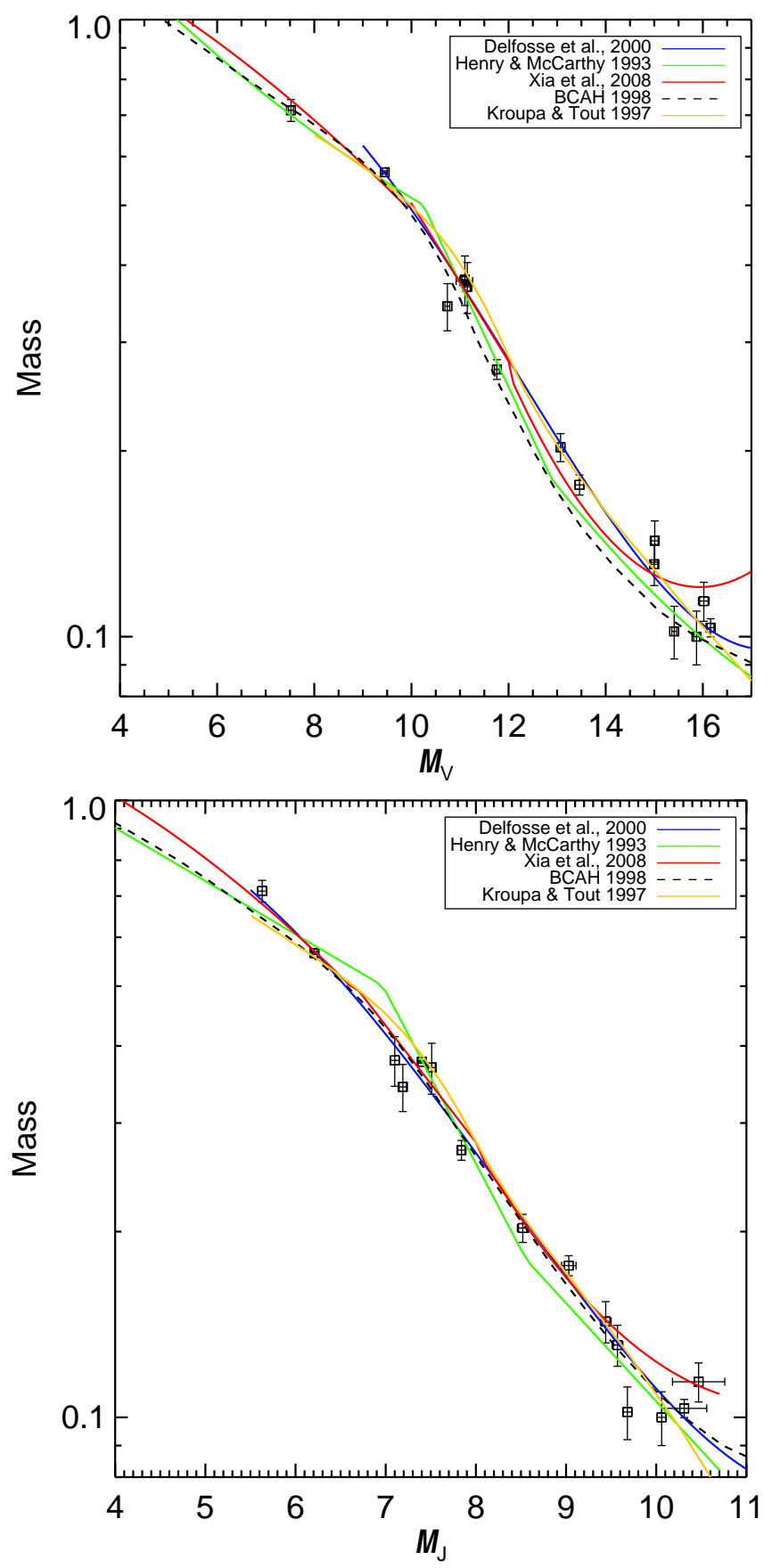

Figure 6.1 Shown are the empirical mass-luminosity relations (MLRs) of Henry \& McCarthy (1993); Kroupa \& Tout (1997); Delfosse et al. (2000) and Xia et al. (2008) along with M dwarfs of known mass from Reid \& Hawley (2005) and the theoretical predictions of Baraffe et al. (1998). The color of each line is given in the legend. Note that in general, the observational and theoretical predictions agree quite well. 
After selecting a MLR, Equation 6.1 is used to transform the $M_{J}$ band LF (Table 5.9) into a MF. This technique is fundamentally different than volume-complete surveys, where a mass is assigned to each individual star and the density is then computed for each mass bin. Instead, the densities measured by the LF are preserved, but expressed in terms of mass. Mathematically, this transformation is identical to the absolute magnitude transformation used to compute the $M_{J}$ LF from the $M_{r} \mathrm{LF}$. The best-fit MF is shown in Figure 6.3 and given in Table 6.2.

The assumed form of the MLR can strongly affect the observed LF. Following the arguments of Kroupa \& Tout (1997) and Reid \& Hawley (2005), I plot the derivative, $d \mathrm{M} / d M$, of the Kroupa \& Tout (1997) and Baraffe et al. (1998) MLRs, along with the measured $M_{J} \mathrm{LF}$. When $d \mathrm{M} / d M$ is large, a given interval in absolute magnitude is distributed into a large range of mass. Thus, a given distribution of mass will produce a peak in the corresponding luminosity function. When $d \mathrm{M} / d M$ is small, a given interval in luminosity is concentrated into a small mass bin, so a given range in mass is widely distributed in absolute magnitude for small values of $d \mathrm{M} / d M$, leading to a deficit in the LF. This behavior is illustrated in Figure 6.2, where the peak in the derivative of the MLRs of Kroupa \& Tout (1997) and Baraffe et al. (1998) roughly corresponds to the maximum in the measured $M_{J} \mathrm{LF}$.

\subsection{Results}

The MF calculated from the $M_{J}$ LF is shown in Figure 6.3 and tabulated in Table 6.2. The MF is fit with two functional forms, a power law (e.g., Tinney, 1993; Kroupa, 2002; Reid et al., 2002) and a log-normal distribution (e.g., Miller \& Scalo, 1979; Chabrier, 2003a; Covey et al., 2008). Since the single power law does not fit the MF data well, I also plot a "broken" power law fit, shown in Figure 6.4. The resulting analytic fits are shown in Figure 6.3 - 6.4 and given in Table 6.3. The MF data and fits are compared to those reported by Covey et al. (2008), Reid \& Gizis (1997), and 


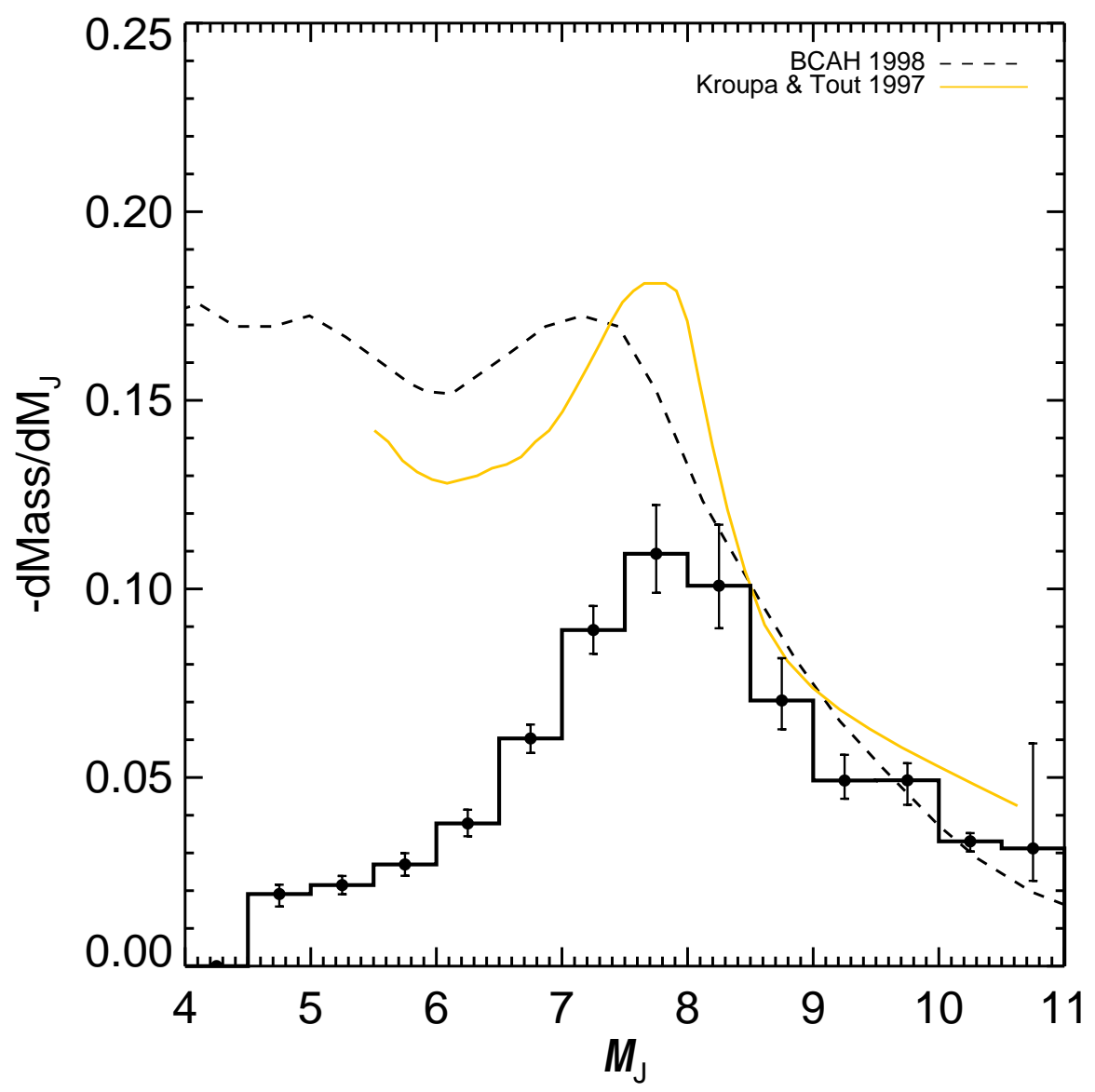

Figure 6.2 Shown is the best fit $J$ band LF from Figure 5.23, along with the derivative of the mass- $M_{J}$ relations of Kroupa \& Tout (1997) (solid yellow line) and Baraffe et al. (1998) (dashed line). Note that the peak in the LF corresponds to a maximum in $d \mathrm{M} / d M_{J}$, indicating that a broad range in mass is being mapped into a small range in absolute magnitude. 
Zheng et al. (2001) in Figures 6.5 - 6.7. Further comparison to the analytic fits of Chabrier (2003a), Kroupa (2002) and Miller \& Scalo (1979) are shown in Figure 6.8.

The MF data from this study are in accordance with the MF data measured by Covey et al. (2008) and Reid \& Gizis (1997). The agreement is slightly better with the Covey et al. (2008) sample, not surprising since the present study and their investigation are derived from similar observations and CMRs. The agreement with Reid \& Gizis (1997) is encouraging, since their volume-complete sample employs trigonometric parallaxes to determine accurate absolute magnitudes and does not rely on a CMR.

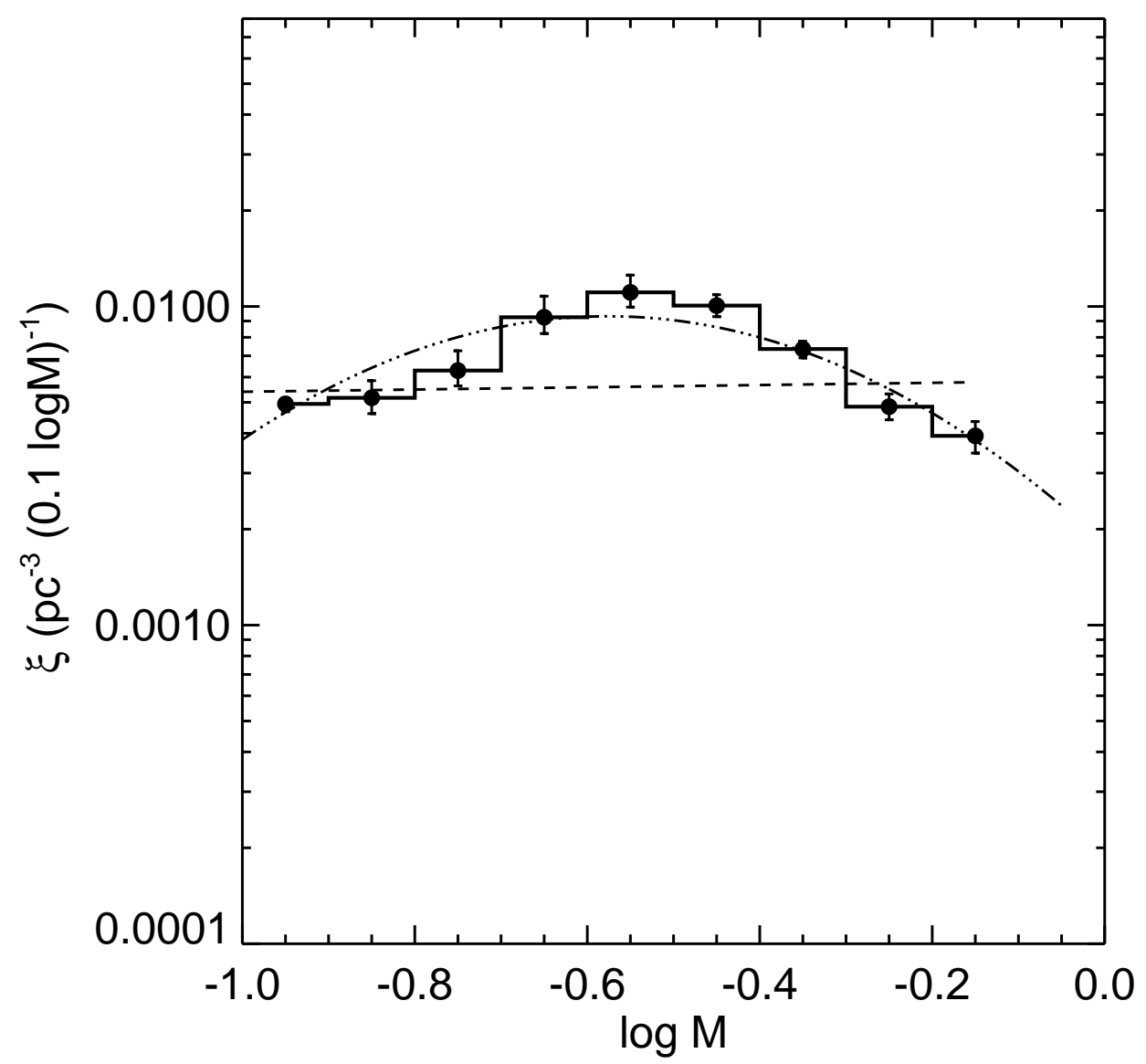

Figure 6.3 Shown is the mass function (filled circles) found by transforming the best fit $M_{J}$ LF from Figure 5.23 with the mass- $M_{J}$ relation of Delfosse et al. (2000). The best fit power law (dashed line) and log-normal (dash-dot line) are listed in Table 6.3. 


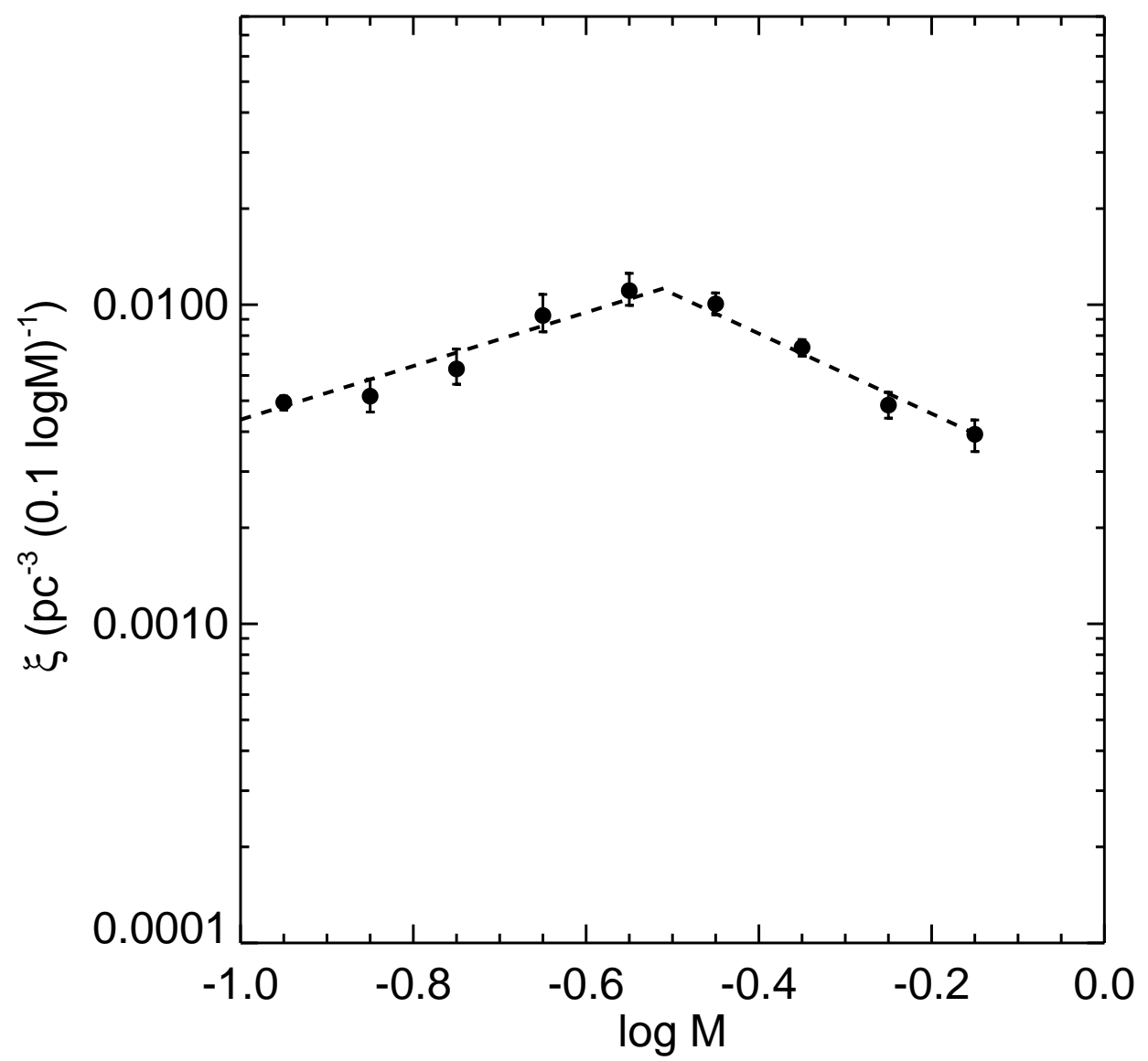

Figure 6.4 Shown is the mass function (filled circles) along with the best fit broken power law (dashed line). The power law break occurs at $\log \mathrm{M}=-0.5$, and provides a better fit than the single power law. The $\alpha$ for each power law segment is quoted in Table 6.3.

As discussed in Covey et al. (2008), some of the present uncertainty in the lowmass IMF may result from comparing analytic fits rather than actual data. As seen in Figures 6.6 and 6.7, there is significant disagreement between the power law fits of Reid \& Gizis (1997) and Zheng et al. (2001) and my own single power law fit. Yet, most of the points agree within the $1-\sigma$ error bars, except for the lowest mass bin. Judged on the basis of functional forms, these studies would not be in agreement. However, the underlying data are clearly similar. This simple example demonstrates the impact of the assumed functional form and fitted mass ranges on the reported 


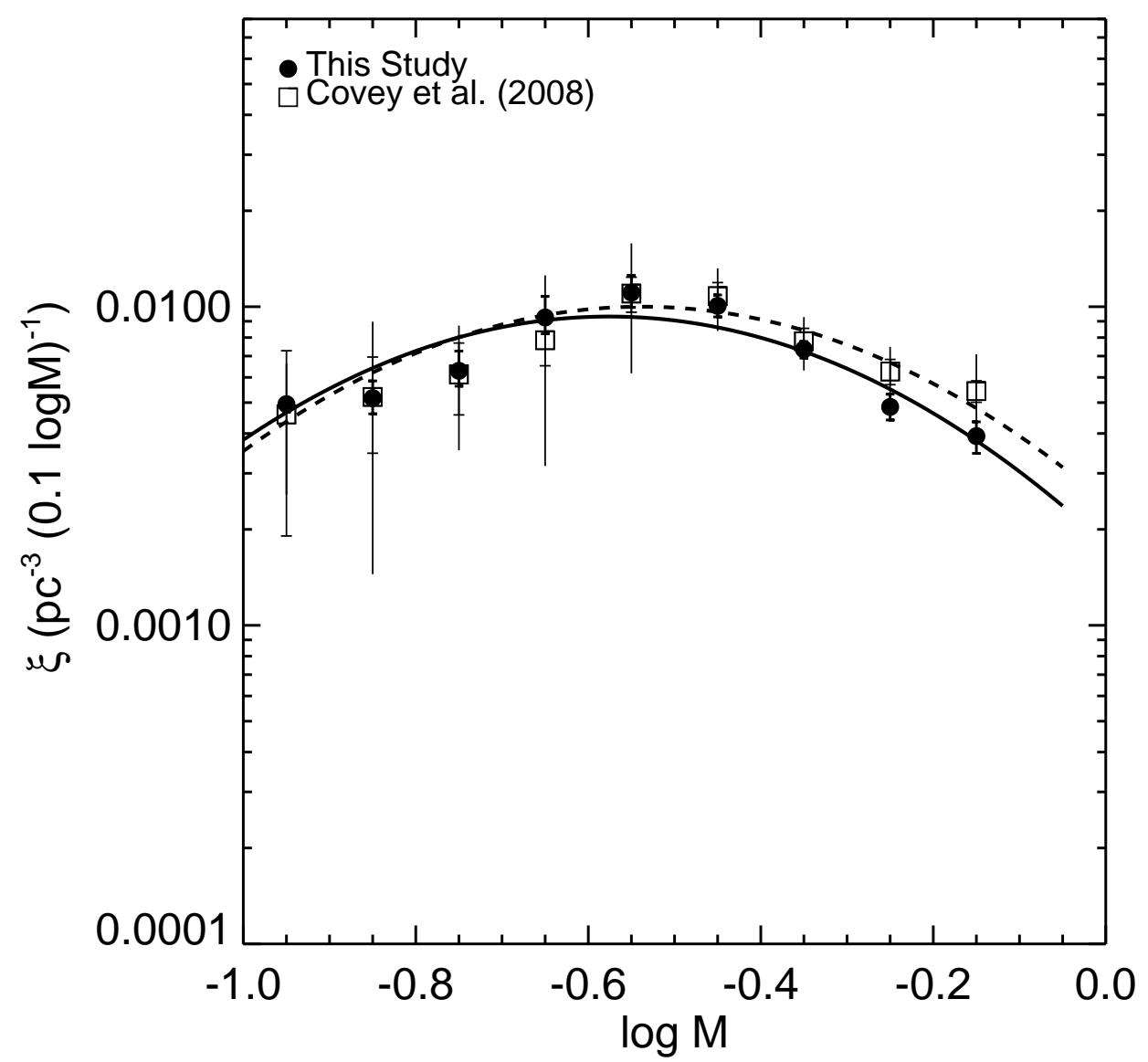

Figure 6.5 Shown is the MF from this dissertation (filled circles) and the MF from Covey et al. (2008) (open squares). My best log-normal fit is overplotted (solid line) and compared to the fit from Covey et al. (2008) (dashed line). Both the data and fits are in agreement; the largest differences lie within the uncertainties. 


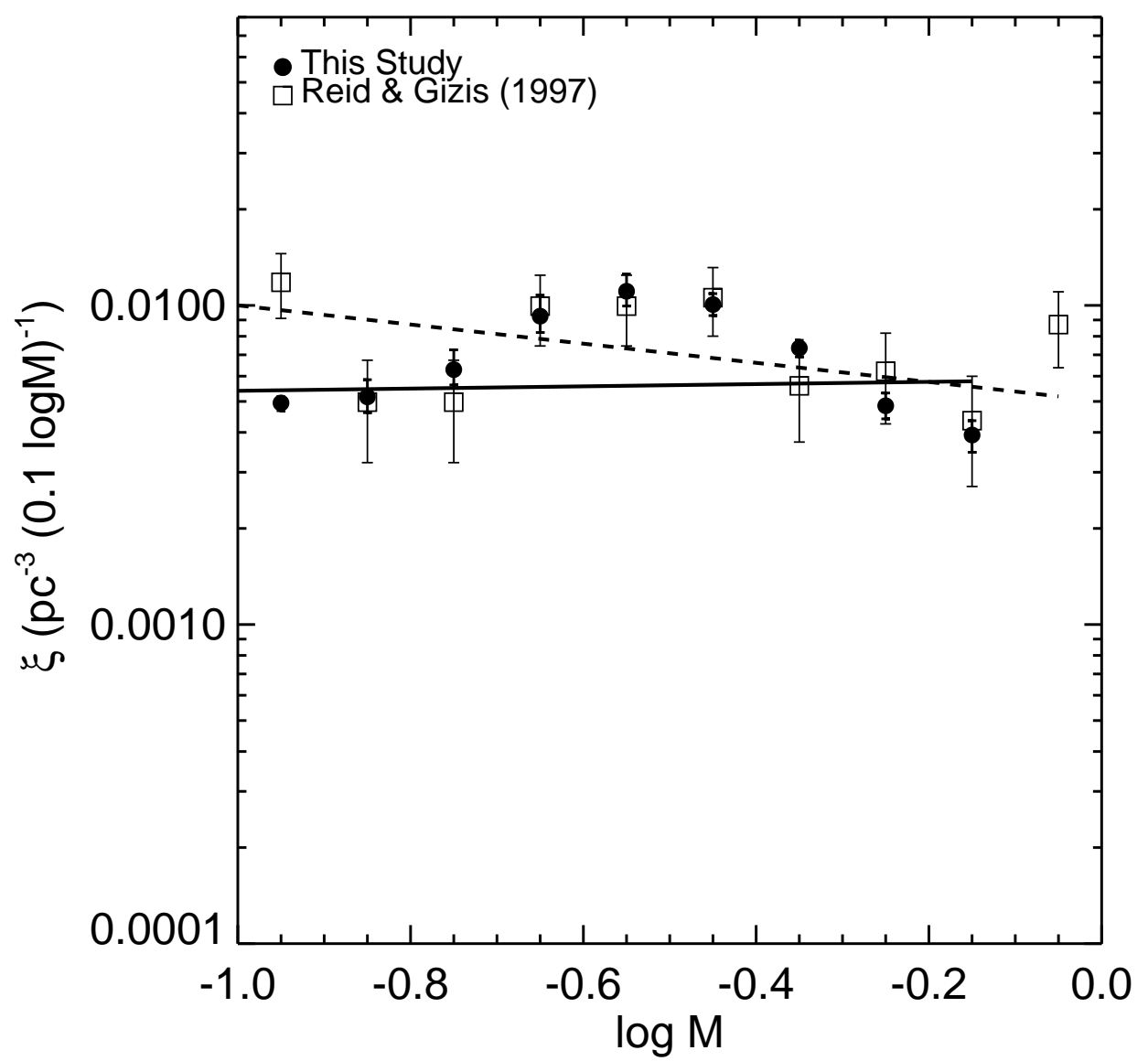

Figure 6.6 Shown is the MF from this dissertation (filled circles) and the MF from Reid \& Gizis (1997) (open squares). Instead of a log-normal fit, a power-law is fit and overplotted (solid line) and compared to the power-law fit from Reid \& Gizis (1997) (dashed line). Again, the data are in excellent agreement, with only the lowest mass bin falling outside the formal $1-\sigma$ error bars. 


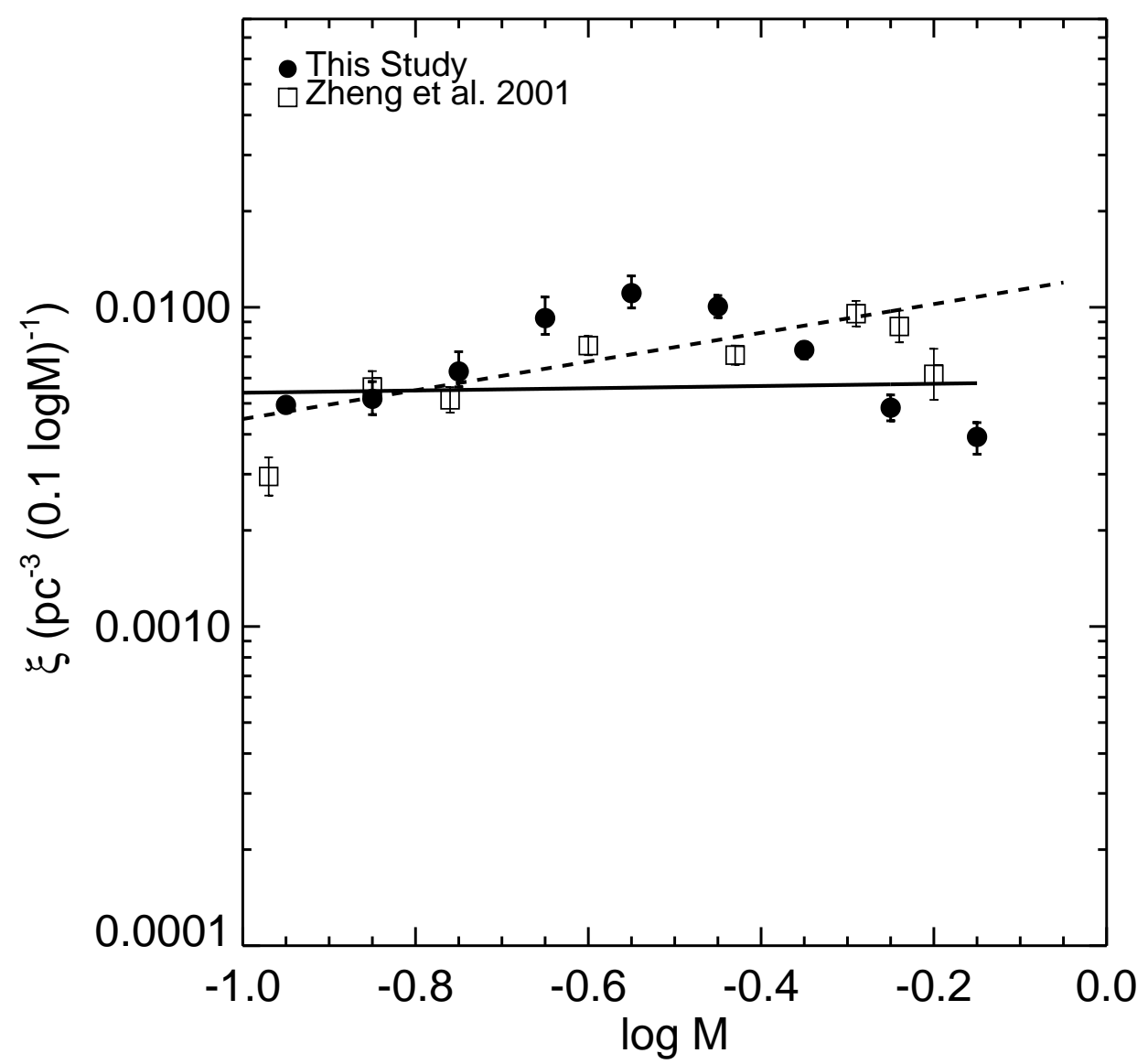

Figure 6.7 Shown is the MF from this dissertation (filled circles) and the MF from Zheng et al. (2001) (open squares). Power law fits are shown for this study (solid line) and the Zheng et al. (2001) results (dashed line). Again, the actual data agree well, despite the discrepancy between the power law fits. 


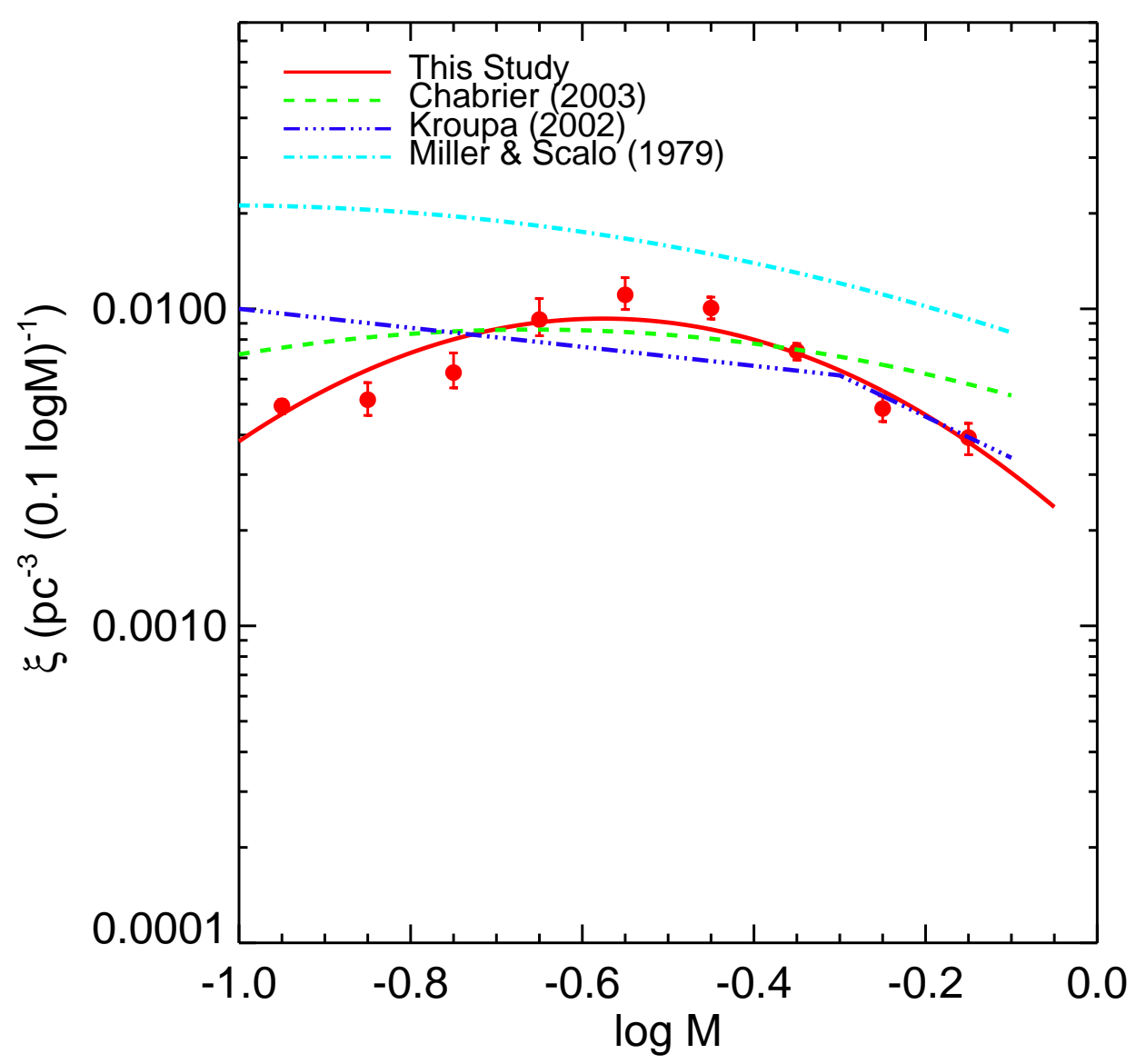

Figure 6.8 Shown is the MF data and best log-normal fit from this study (solid circles and red line), along with the analytic MF fits of Chabrier (2003a) (green dashed line), Kroupa (2002) (dark blue dash-dots line) and Miller \& Scalo (1979) (light blue dashdot line). The Chabrier (2003a) fit is the closest to the present study, but I stress that comparing actual MF data is more valid than comparing analytic fits. 
Table 6.2. Mass Function

\begin{tabular}{llll}
\hline \hline $\log \left(\mathrm{M} / \mathrm{M}_{\odot}\right)$ & $\xi_{\text {Mean }}$ & $\xi_{\text {Max }}$ & $\xi_{\text {Min }}$ \\
\hline-0.15 & 3.925 & 4.353 & 3.465 \\
-0.25 & 4.847 & 5.315 & 4.409 \\
-0.35 & 7.350 & 7.772 & 6.894 \\
-0.45 & 10.059 & 10.883 & 9.289 \\
-0.55 & 11.072 & 12.538 & 9.952 \\
-0.65 & 9.249 & 10.769 & 8.223 \\
-0.75 & 6.292 & 7.257 & 5.631 \\
-0.85 & 5.167 & 5.848 & 4.610 \\
-0.95 & 4.944 & 5.171 & 4.677 \\
\hline
\end{tabular}

Note. - Densities are reported in units of (stars $\left.\mathrm{pc}^{-3} 0.1 \log \mathrm{M}^{-1}\right) \times 10^{-3}$.

mass function. Furthermore, a single power law does not adequately describe the mass function in this regime, as shown in Figure 6.4. Thus, whenever possible, the actual data should be examined, rather than the fitted function, to fairly compare mass functions.

Finally, I note that a single analytic description over a wide range in mass may not be appropriate. Shown in Figure 6.9, are the mass functions of this study, the Reid \& Gizis (1997) sample and the Pleiades (Moraux et al., 2004). The log-normal fit from this study is extended to higher masses and it clearly fails to match the Pleiades MF. Therefore, it is very important to only use the analytic fits over the mass ranges where they are appropriate. Extending analytic fits beyond their quoted bounds can result in significant inaccuracies in the predicted number of stars.

To calculate the local density of $\mathrm{M}$ dwarfs, the mass function shown in Figure 6.3 can be integrated over mass, yielding a density of:

$$
\rho_{\circ}=0.023 \mathrm{M}_{\odot} \mathrm{pc}^{-3}
$$




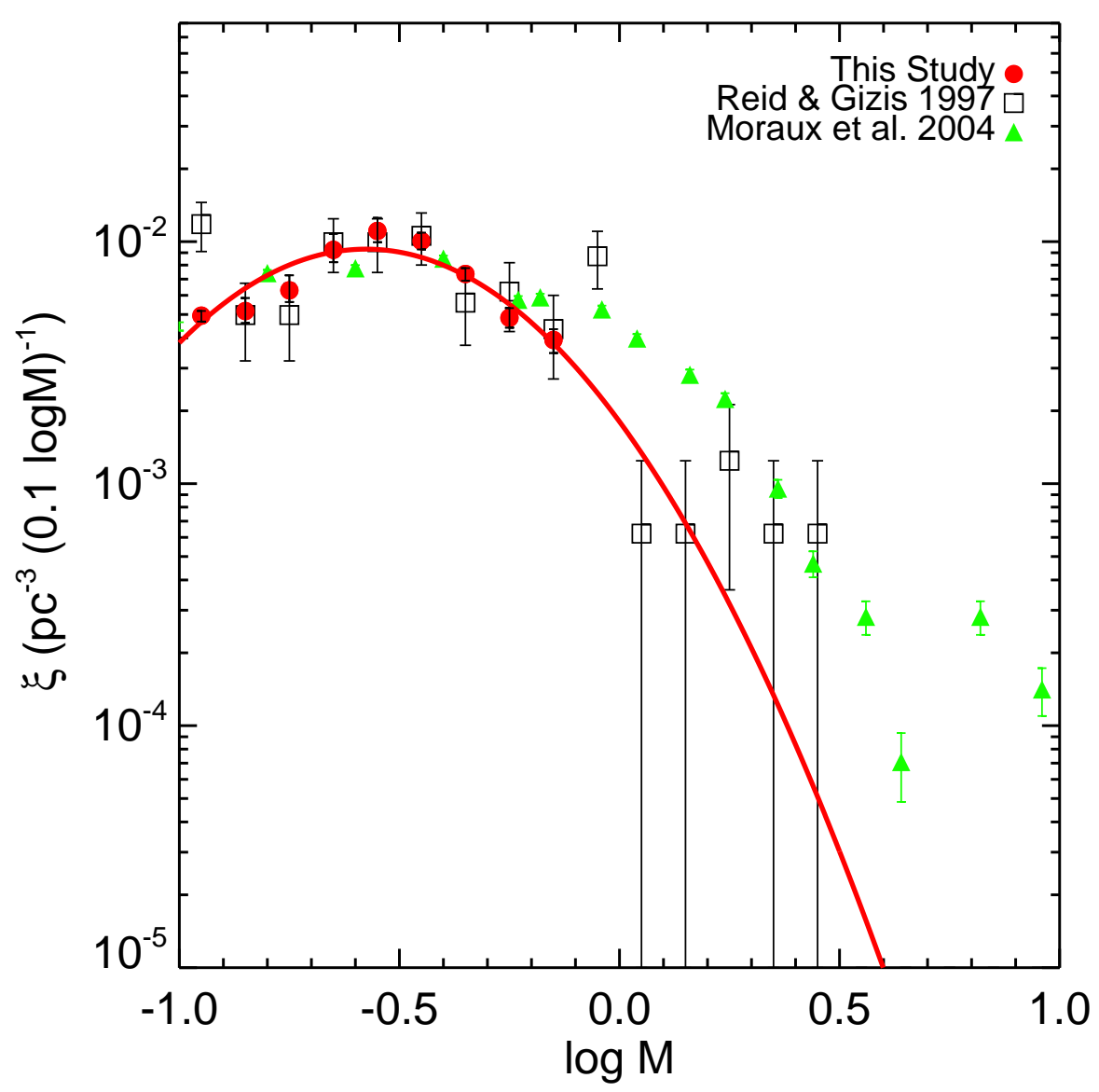

Figure 6.9 Shown is the MF and best log normal fit from this study (red filled circles and solid line), the Reid \& Gizis (1997) MF (open squares) and the Pleiades MF (green triangles, Moraux et al., 2004). The best fit from this study is extended to higher masses and systematically under-predicts the density at high mass. I stress that analytic fits should only be applied over their quoted mass ranges. 
Table 6.3. Mass Function Analytic Fits

\begin{tabular}{lll}
\hline \hline \multicolumn{1}{c}{ Form } & \multicolumn{1}{c}{ Mass Range } & \multicolumn{1}{c}{ Parameter } \\
& & \\
\hline Log-Normal & $-1.0<\log \mathrm{M}<-0.1$ & $\begin{array}{l}C_{\circ}=0.0074 \pm 5.69 \times 10^{-4} \\
\mathrm{M}_{\circ}=0.27 \pm 0.01 \\
\end{array}$ \\
& & $\begin{array}{l}\sigma=0.31 \pm 0.03 \\
\end{array}$ \\
Power Law & $0.1 \mathrm{M}_{\odot}<\mathrm{M}<0.8 \mathrm{M}_{\odot}$ & $\alpha=0.96 \pm 0.14$ \\
Broken Power Law (Low-Mass) & $0.1 \mathrm{M}_{\odot}<\mathrm{M}<0.32 \mathrm{M}_{\odot}$ & $\alpha=0.16 \pm 0.12$ \\
Broken Power Law (High-Mass) & $0.32 \mathrm{M}_{\odot}<\mathrm{M}<0.8 \mathrm{M}_{\odot}$ & $\alpha=2.25 \pm 0.15$ \\
\hline
\end{tabular}

while the surface density is given by:

$$
\Sigma_{\circ}=2 \rho_{\circ}\left(f R_{\circ, \text { Thin }}+(1-f) R_{\circ, \text { Thick }}\right)=13.5 \mathrm{M}_{\odot} \mathrm{pc}^{-2}
$$

with both values comparing favorably to previous results, listed in Table 6.4. The density of $\mathrm{M}$ dwarfs in the solar neighborhood was the subject of intense scrutiny following the dynamical mass estimate of Oort (1960), who determined that a mass density of $0.15 \mathrm{M}_{\odot} \mathrm{pc}^{-3}$ was necessary to explain the motions of nearby stars. Observational densities, found by summing the contributions of local stars, ISM gas and dust, were $\sim 50 \%$ smaller than the dynamical estimates. This discrepancy was seen as indirect evidence of substantial dark matter within the Galactic disk. Since lowmass stars are the dimmest members of the main sequence, they were postulated as a possible source of "normal" dark matter. Resolution was found with new dynamical mass estimates derived from Hipparcos parallax measurements and proper motions (Creze et al., 1998). The modern dynamically inferred density $\left(0.076 \mathrm{M}_{\odot} \mathrm{pc}^{-3}\right)$ is in agreement with the observed value, and the Galactic disk is no longer thought to harbor significant amounts of dark matter. 
Table 6.4. Local Mass Density Estimates

\begin{tabular}{rrll}
\hline \hline \multicolumn{1}{c}{ Study } & \multicolumn{1}{c}{ Mass Range } & $\rho_{\circ}\left(\mathrm{M}_{\odot} \mathrm{pc}^{-3}\right)$ & \multicolumn{1}{c}{$\Sigma_{\circ}\left(\mathrm{M}_{\odot} \mathrm{pc}^{-2}\right)$} \\
\hline Tinney (1993) & $\mathrm{M}<0.4 \mathrm{M}_{\odot}$ & 0.016 & 11.2 \\
Gould et al. (1996) & $0.1 \mathrm{M}_{\odot}<\mathrm{M}<1.6 \mathrm{M}_{\odot}$ & $0.0307 \pm 0.0101$ & $12.4 \pm 1.9$ \\
Zheng et al. (2001) & $0.08 \mathrm{M}_{\odot}<\mathrm{M}<0.6 \mathrm{M}_{\odot}$ & 0.0180 & $14.3 \pm 1.3^{\mathrm{a}}$ \\
Zheng et al. (2001) & $0.08 \mathrm{M}_{\odot}<\mathrm{M}<0.6 \mathrm{M}_{\odot}$ & 0.0153 & $12.2 \pm 1.6^{\mathrm{b}}$ \\
Reid et al. (2002) & $0.08 \mathrm{M}_{\odot}<\mathrm{M} \lesssim 3 \mathrm{M}_{\odot}$ & 0.030 & $\ldots$ \\
This Study & $0.1 \mathrm{M}_{\odot}<\mathrm{M}<0.8 \mathrm{M}_{\odot}$ & $0.0232_{-0.00242}^{+0.00259}$ & $13.52_{-1.40}^{+1.51}$ \\
\hline
\end{tabular}

${ }^{a}$ Computed with CMR(1) from Zheng et al. (2001) (no metallicity dependence).

${ }^{\mathrm{b}}$ Computed with $\mathrm{CMR}(2)$ from Zheng et al. (2001) (metallicity dependence included).

\subsection{Conclusions}

I report on a new measurement of the stellar mass function, from $0.1 \mathrm{M}_{\odot}<\mathrm{M}<0.8$ $\mathrm{M}_{\odot}$. The measured mass function is derived from the local luminosity function (see Chapter 5), and agrees with previous determinations from photometric and volume complete samples. The local mass density is integrated and found to be in broad agreement with previous studies. The best-fit power law and log-normal distributions are reported in Table 6.3. I re-emphasize the discussion of Covey et al. (2008), stressing the importance of comparing actual MF data points rather than analytic fits.

Mass, age, and metallicity can all affect the luminosity of a given star. Since this analysis requires an accurate transformation from luminosity into mass, I investigated the effects of age and metallicity on the analysis. Over the mass range probed, there is no luminosity evolution over the lifetime of the Galaxy (Laughlin et al., 1997), avoiding complications due to age. As discussed in $\S 6.2$, metallicity does not significantly affect the assumed mass- $M_{J}$ relation. Thus, the luminosity of a given 
low-mass star should be a good tracer of its mass.

In the future, this analysis technique will be applied to study variations of the MF with location in the Milky Way, especially in the thick disk. These investigations will yield insight into the formation of the thick disk population, which is still uncertain. 


\section{Chapter 7}

\section{CONCLUSIONS}

The goal of this thesis was to characterize the low-mass stellar population near the Sun, using the unprecedented spectroscopic and photometric observations of the Sloan Digital Sky Survey. The studies described in the previous chapters surveyed the mean spectroscopic properties of M dwarfs, examined the kinematic structure of the thin and thick disks, measured the shape of the main sequence at low-luminosities, and determined the stellar density structure of the local Milky Way, while simultaneously measuring the low-mass stellar luminosity and mass functions. I detail the notable conclusions of this dissertation below:

- I constructed medium resolution spectral templates, based on thousands of coadded SDSS spectral observations of low-mass dwarfs. These templates are suitable for radial velocity analysis, with external accuracy of $\lesssim 10 \mathrm{~km} \mathrm{~s}^{-1}$ and internal precision of $\lesssim 1 \mathrm{~km} \mathrm{~s}^{-1}$. The spectral templates were divided by activity, as traced by the $\mathrm{H} \alpha$ emission line, to examine the effects of magnetic activity on the spectroscopic features of low-mass dwarfs. I showed that activity does affect some $\mathrm{TiO}$ and $\mathrm{CaH}$ molecular bandheads, as well as the $u-g$ colors of M dwarfs. Finally, the average ugriz colors and molecular bandhead indices were reported as a function of spectral type.

- Using a targeted set of SDSS spectroscopic and photometric observations along one line of sight, I studied the kinematic structure of the thin and thick disks, using low-mass stars as tracers of these populations. The radial velocities measured from the spectra were combined with proper motions and distances esti- 
mated from photometric parallaxes to construct full $U V W$ space motions. The mean $U V W$ velocities and their dispersions were measured as a function of distance from the Galactic Plane, and compared to model predictions for the Milky Way. The model may be underestimating the some velocity dispersions, particularly in the $W$ direction. This is probably due to the assumed age-velocity dispersion relation. Finally, the thin and thick disk populations were kinematically separated to examine alternative observational differences in metallicity, colors, and activity. I found possible evidence for an older population in the activity distribution, but the difference between the thin and thick disks was within the error bars.

- Since all of the stars observed by SDSS are fainter than $V \sim 12$, they do not have well-measured trigonometric parallaxes from Hipparcos (although some faint stars have good parallaxes from targeted surveys such as USNO). Thus, the absolute magnitude (and distance) of a star must be estimated from its color or spectral type. The native ugriz photometric system of the SDSS has multiple CMRs defined, each using a variety of sources to calibrate the absolute magnitude. Using a sample of nearby stars with measured parallaxes and ugrizJHK photometry, I constructed new CMR relations to estimate the absolute magnitudes of low-mass stars in the SDSS system. I also defined absolute magnitude transformations, which map the absolute magnitude from one filter into another. These relations were used to transform the observed $M_{r}$ luminosity function into $M_{J}$.

- Using a dataset of over 15 million low-mass dwarfs with ugriz photometry, I measured the $M_{r}$ luminosity function in the local Milky Way. Since the usual $V_{\text {eff }}$ formalism would be computationally prohibitive, I introduced a novel method for measuring the LF in large sky surveys. Stellar densities maps were computed as a function of Galactic radius $(R)$ and Galactic height $(Z)$. A map was 
constructed for every 0.5 magnitudes in $M_{r}$. A Galaxy density model was fit to these maps, which estimates the scale heights and lengths of the thin and thick disks, along with the local density of each 0.5 absolute magnitude slice. These local densities were then plotted as a function of absolute magnitude, yielding the luminosity function of low-mass dwarfs. The Galactic structure parameters and LF were compared to previous studies. In particular, I showed that this new formalism reproduced the results of the $V_{\text {eff }}$ technique and compared favorably to the system LF measured for nearby trig parallax stars. The errors were quantified using a Monte Carlo analysis, which measures the effects of Malmquist bias and unresolved binaries. Systematic CMR errors, due to incorrect CMRs or metallicity were also quantified, and shown to have a major influence on the resulting $\mathrm{LF}$.

- Using empirical mass-luminosity relations, the measured LF was transformed into a mass function. The mass function is important to both star formation theory and Galactic evolution, and my measurement of the low-mass MF places the strongest observational limits on it to date. I compared to previous MFs derived from photometric and trig parallax samples, and showed that the data agree over the overlapping mass ranges. The uncertainties associated in fitting a mathematical form to the MF were discussed. The best-fit MF was a lognormal distribution with $\mathrm{M}_{\circ}=0.27$, but a broken power law also fit the data reasonably well. Finally, the local mass density was calculated and compared to previous observational measurements. The main source of uncertainty in both the MF and LF study was the assumed CMR, which dominates the shape of stellar density maps. Future parallax surveys, such as GAIA and LSST, should improve this situation, obtaining trigonometric parallaxes down to $V \sim 20$ mag. 


\section{BIBLIOGRAPHY}

Abazajian, K., et al. 2003, AJ, 126, 2081

—. 2004, AJ, 128, 502

-. 2005, AJ, 129, 1755

Adelman-McCarthy, J. K., et al. 2006, ApJS, 162, 38

-. 2007a, ApJS, 172, 634

-. 2007b, ArXiv e-prints, 707

Allard, F., Hauschildt, P. H., Alexander, D. R., \& Starrfield, S. 1997, ARA\&A, 35, 137

Allende Prieto, C., Barklem, P. S., Lambert, D. L., \& Cunha, K. 2004, A\&A, 420, 183

Allred, J. C., Hawley, S. L., Abbett, W. P., \& Carlsson, M. 2006, ApJ, 644, 484

Amado, P. J., \& Byrne, P. B. 1997, A\&A, 319, 967

Bahcall, J. N., \& Soneira, R. M. 1980, ApJS, 44, 73

Baraffe, I., Chabrier, G., Allard, F., \& Hauschildt, P. H. 1998, A\&A, 337, 403

Bell, E. F., \& de Jong, R. S. 2001, ApJ, 550, 212

Belokurov, V., et al. 2006, ApJL, 642, L137

—. 2007, ApJ, 654, 897

Bensby, T., Feltzing, S., \& Lundström, I. 2003, A\&A, 410, 527

Beuzit, J.-L. et al. 2004, A\&A, 425, 997

Bienayme, O., Robin, A. C., \& Creze, M. 1987, A\&A, 180, 94

Binney, J., Dehnen, W., \& Bertelli, G. 2000, MNRAS, 318, 658

Binney, J., Gerhard, O., \& Spergel, D. 1997, MNRAS, 288, 365 
Bochanski, J. J., Hawley, S. L., Reid, I. N., Covey, K. R., West, A. A., Tinney, C. G., \& Gizis, J. E. 2005, AJ, 130, 1871

Bochanski, J. J., Munn, J. A., Hawley, S. L., West, A. A., Covey, K. R., \& Schneider, D. P. 2007a, AJ, 134, 2418

Bochanski, J. J., West, A. A., Hawley, S. L., \& Covey, K. R. 2007b, AJ, 133, 531

Bok, B. J. 1937, The distribution of the stars in space (Chicago: University of Chicago Press, 1937)

Bok, B. J., \& Macrae, D. A. 1941, New York Academy Sciences Annals, 42, 219

Bonfils, X., Delfosse, X., Udry, S., Santos, N. C., Forveille, T., \& Ségransan, D. 2005, A\&A, 442, 635

Bosma, A. 1978, PhD thesis, PhD Thesis, Groningen Univ., (1978)

Brook, C. B., Kawata, D., Gibson, B. K., \& Freeman, K. C. 2004, ApJ, 612, 894

Burgasser, A. J., \& Kirkpatrick, J. D. 2006, ApJ, 645, 1485

Burgasser, A. J., Reid, I. N., Siegler, N., Close, L., Allen, P., Lowrance, P., \& Gizis, J. 2007, in Protostars and Planets V, ed. B. Reipurth, D. Jewitt, \& K. Keil, 427-441

Burgasser, A. J., Vrba, F. J., Lépine, S., Munn, J. A., Luginbuhl, C. B., Henden, A. A., Guetter, H. H., \& Canzian, B. C. 2008, ApJ, 672, 1159

Burrows, A., Hubbard, W. B., Saumon, D., \& Lunine, J. I. 1993, ApJ, 406, 158

Buser, R., Rong, J., \& Karaali, S. 1999, A\&A, 348, 98

Camm, G. L. 1950, MNRAS, 110, 305

-. 1952, MNRAS, 112, 155

Chabrier, G. 2001, ApJ, 554, 1274

-. 2003a, PASP, 115, 763

-. 2003b, ApJL, 586, L133

Chabrier, G., Baraffe, I., Allard, F., \& Hauschildt, P. H. 2005, ArXiv Astrophysics e-prints

Chen, B., et al. 2001, ApJ, 553, 184 
Chiba, M., \& Beers, T. C. 2000, AJ, 119, 2843

Chiu, K., Fan, X., Leggett, S. K., Golimowski, D. A., Zheng, W., Geballe, T. R., Schneider, D. P., \& Brinkmann, J. 2006, AJ, 131, 2722

Clem, J. L. 2006, PhD thesis, University of Victoria (Canada)

Clem, J. L., Vanden Berg, D. A., \& Stetson, P. B. 2008, AJ, 135, 682

Cohen, M. 1995, ApJ, 444, 874

Covey, K. C., et al. 2007a, AJ, in preparation

-. 2008, AJ, in preparation

Covey, K. R., et al. 2007b, AJ, 134, 2398

Creze, M., Chereul, E., Bienayme, O., \& Pichon, C. 1998, A\&A, 329, 920

Cruz, K. L., \& Reid, I. N. 2002, AJ, 123, 2828

Cruz, K. L., et al. 2007, AJ, 133, 439

Cutri, R. M., et al. 2003, VizieR Online Data Catalog, 2246, 0

Dahn, C. C., Liebert, J., \& Harrington, R. S. 1986, AJ, 91, 621

Dahn, C. C., Liebert, J., Harris, H. C., \& Guetter, H. H. 1995, in The Bottom of the Main Sequence - and Beyond, Proceedings of the ESO Workshop Held in Garching, Germany, 10-12 August 1994, edited by Christopher G. Tinney. Springer-Verlag Berlin Heidelberg New York. Also ESO Astrophysics Symposia, 1995., p.239, ed. C. G. Tinney, 239-+

Dahn, C. C., et al. 2002, AJ, 124, 1170

Dalcanton, J. J., Spergel, D. N., \& Summers, F. J. 1997, ApJ, 482, 659

Davenport, J. R. A., Bochanski, J. J., Covey, K. R., Hawley, S. L., West, A. A., \& Schneider, D. P. 2007, AJ, 134, 2430

Davenport, J. R. A., West, A. A., Matthiesen, C. K., Schmieding, M., \& Kobelski, A. 2006, PASP, 118, 1679

de Vaucouleurs, G., \& Pence, W. D. 1978, AJ, 83, 1163

Dehnen, W., \& Binney, J. J. 1998, MNRAS, 298, 387 
Delfosse, X. et al. 2004, in Astronomical Society of the Pacific Conference Series, Vol. 318, Spectroscopically and Spatially Resolving the Components of the Close Binary Stars, ed. R. W. Hilditch, H. Hensberge, \& K. Pavlovski, 166-174

Delfosse, X., Forveille, T., Ségransan, D., Beuzit, J.-L., Udry, S., Perrier, C., \& Mayor, M. 2000, A\&A, 364, 217

Delhaye, J. 1965, in Galactic Structure, ed. A. Blaauw \& M. Schmidt, 61-+

Duquennoy, A., \& Mayor, M. 1991, A\&A, 248, 485

Eisenstein, D. J., et al. 2001, AJ, 122, 2267

-. 2005, ApJ, 633, 560

Elmegreen, B. G., \& Mathieu, R. D. 1983, MNRAS, 203, 305

ESA. 1997, VizieR Online Data Catalog, 1239, 0

Faber, S. M., Burstein, D., Tinsley, B., \& King, I. R. 1976, AJ, 81, 45

Fan, X. 1999, AJ, 117, 2528

Fan, X., et al. 1996, AJ, 112, 628

-. 2000, AJ, 119, 928

-. 2006, AJ, 131, 1203

Feast, M. W., \& Catchpole, R. M. 1997, MNRAS, 286, L1

Felten, J. E. 1976, ApJ, 207, 700

Feltzing, S., Bensby, T., \& Lundström, I. 2003, A\&A, 397, L1

Fischer, D. A., \& Marcy, G. W. 1992, ApJ, 396, 178

Freeman, K. C. 1970, ApJ, 160, 811

Frieman, J. A., et al. 2007, AJ, submitted

-. 2008, AJ, 135, 338

Fruchter, A. S., \& Hook, R. N. 2002, PASP, 114, 144

Fuhrmann, K. 1998, A\&A, 338, 161

Fukugita, M., Ichikawa, T., Gunn, J. E., Doi, M., Shimasaku, K., \& Schneider, D. P. 
1996, AJ, 111, 1748

Giclas, H. L., Burnham, R., \& Thomas, N. G. 1971, Lowell proper motion survey Northern Hemisphere. The G numbered stars. 8991 stars fainter than magnitude 8 with motions < 0".26/year (Flagstaff, Arizona: Lowell Observatory, 1971)

Gilmore, G., \& Reid, N. 1983, MNRAS, 202, 1025

Girardi, L., Grebel, E. K., Odenkirchen, M., \& Chiosi, C. 2004, A\&A, 422, 205

Gizis, J. E. 1997, AJ, 113, 806

Gizis, J. E., Monet, D. G., Reid, I. N., Kirkpatrick, J. D., Liebert, J., \& Williams, R. J. 2000, AJ, 120, 1085

Gizis, J. E., Reid, I. N., \& Hawley, S. L. 2002, AJ, 123, 3356

Gliese, W., \& Jahreiss, H. 1991, Preliminary Version of the Third Catalogue of Nearby Stars, Tech. rep.

Golimowski, D. A., et al. 2004, AJ, 127, 3516

-. 2009, AJ, in preparation

Gomez, A. E., Grenier, S., Udry, S., Haywood, M., Meillon, L., Sabas, V., Sellier, A., \& Morin, D. 1997, in ESA SP-402: Hipparcos - Venice '97, 621-624

Gould, A., Bahcall, J. N., \& Flynn, C. 1996, ApJ, 465, 759

—. 1997, ApJ, 482, 913

Governato, F., Willman, B., Mayer, L., Brooks, A., Stinson, G., Valenzuela, O., Wadsley, J., \& Quinn, T. 2007, MNRAS, 374, 1479

Griffin, R. F., Griffin, R. E. M., Gunn, J. E., \& Zimmerman, B. A. 1988, AJ, 96, 172

Güdel, M., Audard, M., Skinner, S. L., \& Horvath, M. I. 2002, ApJL, 580, L73

Gunn, J. E., Griffin, R. F., Griffin, R. E. M., \& Zimmerman, B. A. 1988, AJ, 96, 198

Gunn, J. E., et al. 1998, AJ, 116, 3040

-. 2006, AJ, 131, 2332

Hanson, R. B. 1979, MNRAS, 186, 875

Hartwick, F. D. A. 1977, ApJ, 214, 778 
Hauschildt, P., \& Baron, E. 2005, Memorie della Societa Astronomica Italiana Supplement, 7,140

Hauschildt, P. H., Allard, F., \& Baron, E. 1999, ApJ, 512, 377

Hawkins, M. R. S., \& Bessell, M. S. 1988, MNRAS, 234, 177

Hawley, S. L., Gizis, J. E., \& Reid, I. N. 1996, AJ, 112, 2799

Hawley, S. L., \& Pettersen, B. R. 1991, ApJ, 378, 725

Hawley, S. L., Tourtellot, J. G., \& Reid, I. N. 1999, AJ, 117, 1341

Hawley, S. L., et al. 2002, AJ, 123, 3409

Henry, T. J., Kirkpatrick, J. D., \& Simons, D. A. 1994, AJ, 108, 1437

Henry, T. J., \& McCarthy, Jr., D. W. 1990, ApJ, 350, 334

—. 1993, AJ, 106, 773

Henry, T. J., Subasavage, J. P., Brown, M. A., Beaulieu, T. D., Jao, W.-C., \& Hambly, N. C. $2004, \mathrm{AJ}, 128,2460$

Herbst, T. M., Thompson, D., Fockenbrock, R., Rix, H.-W., \& Beckwith, S. V. W. 1999, ApJL, 526, L17

Hogg, D. W., Finkbeiner, D. P., Schlegel, D. J., \& Gunn, J. E. 2001, AJ, 122, 2129

Ibata, R. A., Gilmore, G., \& Irwin, M. J. 1994, Nature, 370, 194

Ivezić, Ž., et al. 2003, Memorie della Societa Astronomica Italiana, 74, 978

—. 2004, Astronomische Nachrichten, 325, 583

—. 2007, AJ, 134, 973

Ivezic, Z., et al. 2008, ArXiv e-prints, 804

Johnson, D. R. H., \& Soderblom, D. R. 1987, AJ, 93, 864

Juric, M., et al. 2005, ArXiv Astrophysics e-prints

Jurić, M., et al. 2008, ApJ, 673, 864

Kaib, N. A., \& Quinn, T. 2007, ArXiv e-prints, 707

Kapteyn, J. C. 1902, Publications of the Kapteyn Astronomical Laboratory Gronin- 
gen, 11,1

-. 1914, ApJ, 40, 43

Kapteyn, J. C., \& van Rhijn, P. J. 1920, ApJ, 52, 23

Kerber, L. O., Javiel, S. C., \& Santiago, B. X. 2001, A\&A, 365, 424

Kerr, F. J., \& Lynden-Bell, D. 1986, MNRAS, 221, 1023

Kilic, M. et al. 2006, AJ, 131, 582

King, I., Gilmore, G., \& van der Kruit, P. C., eds. 1990, The Milky Way As Galaxy

Kirkpatrick, J. D., Henry, T. J., \& McCarthy, D. W. 1991, ApJS, 77, 417

Kirkpatrick, J. D., Henry, T. J., \& Simons, D. A. 1995, AJ, 109, 797

Kirkpatrick, J. D., et al. 1999, ApJ, 519, 802

Knapp, G. R., et al. 2004, AJ, 127, 3553

Kroupa, P. 2002, Science, 295, 82

Kroupa, P., Gilmore, G., \& Tout, C. A. 1991, MNRAS, 251, 293

Kroupa, P., \& Tout, C. A. 1997, MNRAS, 287, 402

Kroupa, P., Tout, C. A., \& Gilmore, G. 1990, MNRAS, 244, 76

—. 1993, MNRAS, 262, 545

Kuiper, G. P. 1942, ApJ, 95, 201

Lacy, C. H., Moffett, T. J., \& Evans, D. S. 1976, ApJS, 30, 85

Lada, C. J. 2006, ApJL, 640, L63

Larsen, J. A., \& Humphreys, R. M. 2003, AJ, 125, 1958

Laughlin, G., Bodenheimer, P., \& Adams, F. C. 1997, ApJ, 482, 420

Leggett, S. K., et al. 2000, ApJL, 536, L35

Lejeune, T., Cuisinier, F., \& Buser, R. 1998, A\&AS, 130, 65

Lépine, S., Rich, R. M., \& Shara, M. M. 2003a, AJ, 125, 1598

-. 2003b, AJ, 125, 1598 
Lépine, S., \& Shara, M. M. 2005, AJ, 129, 1483

Lupton, R., Gunn, J. E., Ivezić, Z., Knapp, G. R., \& Kent, S. 2001, in Astronomical Society of the Pacific Conference Series, Vol. 238, Astronomical Data Analysis Software and Systems X, ed. F. R. Harnden, Jr., F. A. Primini, \& H. E. Payne, $269-+$

Lutz, T. E., \& Kelker, D. H. 1973, PASP, 85, 573

Lutz, T. E., \& Upgren, A. R. 1980, AJ, 85, 1390

Luyten, W. J. 1923, Lick Observatory Bulletin, 11, 1

-. 1939, Publications of the Astronomical Observatory University of Minnesota, 2, 121

—. 1941, New York Academy Sciences Annals, 42, 201

—. 1968, MNRAS, 139, 221

—. 1979, LHS catalogue. A catalogue of stars with proper motions exceeding 0"5 annually (Minneapolis: University of Minnesota, 1979, 2nd ed.)

Majewski, S. R. 1993, ARA\&A, 31, 575

Majewski, S. R., Skrutskie, M. F., Weinberg, M. D., \& Ostheimer, J. C. 2003, ApJ, 599,1082

Makarov, V. V., Odenkirchen, M., \& Urban, S. 2000, A\&A, 358, 923

Malmquist, K. G. 1936, Stockholm Obs. Medd., 26

Martín, E. L. 1999, MNRAS, 302, 59

Martini, P., \& Osmer, P. S. 1998, AJ, 116, 2513

Mathieu, R. D., Latham, D. W., Griffin, R. F., \& Gunn, J. E. 1986, AJ, 92, 1100

Mauas, P. J. D., \& Falchi, A. 1994, A\&A, 281, 129

Mauas, P. J. D., Falchi, A., Pasquini, L., \& Pallavicini, R. 1997, A\&A, 326, 249

McCuskey, S. W. 1966, Vistas in Astronomy, 7, 141

Mendez, R. A., \& van Altena, W. F. 1996, AJ, 112, 655

Miller, G. E., \& Scalo, J. M. 1979, ApJS, 41, 513 
Minkowski, R. L., \& Abell, G. O. 1963, The National Geographic Society-Palomar Observatory Sky Survey (Basic Astronomical Data: Stars and stellar systems, edited by K. A. Strand. Published by the University of Chicago Press, Chicago, IL USA, 1968, p.481), 481-+

Moffett, T. J., \& Bopp, B. W. 1976, ApJS, 31, 61

Monet, D. G., Dahn, C. C., Vrba, F. J., Harris, H. C., Pier, J. R., Luginbuhl, C. B., \& Ables, H. D. 1992, AJ, 103, 638

Moraux, E., Kroupa, P., \& Bouvier, J. 2004, A\&A, 426, 75

Munn, J. A., et al. 2004, AJ, 127, 3034

Newberg, H. J., et al. 2002, ApJ, 569, 245

Ng, Y. K., Bertelli, G., Chiosi, C., \& Bressan, A. 1997, A\&A, 324, 65

Nidever, D. L., Marcy, G. W., Butler, R. P., Fischer, D. A., \& Vogt, S. S. 2002, ApJS, 141,503

Norris, J. E. 1999, Ap\&SS, 265, 213

O’Connell, D. J. K. 1958, Ricerche Astronomiche, 5

Ojha, D. K. 2001, MNRAS, 322, 426

Ojha, D. K., Bienaymé, O., Mohan, V., \& Robin, A. C. 1999, A\&A, 351, 945

Ojha, D. K., Bienayme, O., Robin, A. C., Creze, M., \& Mohan, V. 1996, A\&A, 311, 456

Oort, J. H. 1932, Bull. Astron. Inst. Netherlands, 6, 249

—. 1960, Bull. Astron. Inst. Netherlands, 15, 45

Padmanabhan, N., et al. 2008, ApJ, 674, 1217

Parenago, P. P. 1950, AZh, 27, 41

Paulson, D. B., Sneden, C., \& Cochran, W. D. 2003, AJ, 125, 3185

Pernechele, C., Poletto, L., Nicolosi, P., \& Naletto, G. 1996, Optical Engineering, 35, 1503

Perryman, M. A. C. et al. 2001, A\&A, 369, 339 
Pettersen, B. R., \& Hawley, S. L. 1989, A\&A, 217, 187

Phleps, S., Meisenheimer, K., Fuchs, B., \& Wolf, C. 2000, A\&A, 356, 108

Pier, J. R., Munn, J. A., Hindsley, R. B., Hennessy, G. S., Kent, S. M., Lupton, R. H., \& Ivezić, Ž. 2003, AJ, 125, 1559

Pirzkal, N. et al. 2005, ApJ, 622, 319

Press, W. H., Teukolsky, S. A., Vetterling, W. T., \& Flannery, B. P. 1992, Numerical recipes in FORTRAN. The art of scientific computing (Cambridge: University Press, —c1992, 2nd ed.)

Ratnatunga, K. U., \& Upgren, A. R. 1997, ApJ, 476, 811

Rauscher, E., \& Marcy, G. W. 2006, PASP, 118, 617

Raymond, S. N., et al. 2003, AJ, 125, 2621

Reid, I. N. 1997, AJ, 114, 161

Reid, I. N., \& Cruz, K. L. 2002, AJ, 123, 2806

Reid, I. N. et al. 2003a, AJ, 126, 3007

-. 2003b, AJ, 125, 354

Reid, I. N., \& Gizis, J. E. 1997, AJ, 113, 2246

Reid, I. N., Gizis, J. E., Cohen, J. G., Pahre, M. A., Hogg, D. W., Cowie, L., Hu, E., \& Songaila, A. 1997, PASP, 109, 559

Reid, I. N., Gizis, J. E., \& Hawley, S. L. 2002, AJ, 124, 2721

Reid, I. N., \& Hawley, S. L. 2005, New light on dark stars : red dwarfs, low-mass stars, brown dwarfs (New Light on Dark Stars Red Dwarfs, Low-Mass Stars, Brown Stars, by I.N. Reid and S.L. Hawley. Springer-Praxis books in astrophysics and astronomy. Praxis Publishing Ltd, 2005. ISBN 3-540-25124-3)

Reid, I. N., Hawley, S. L., \& Gizis, J. E. 1995a, AJ, 110, 1838

Reid, I. N. et al. 1999, ApJ, 521, 613

Reid, I. N., \& Mahoney, S. 2000, MNRAS, 316, 827

Reid, I. N., et al. 1991, PASP, 103, 661 
-. 2004, AJ, 128, 463

Reid, N. 1982, MNRAS, 201, 51

Reid, N. 1993, in ASP Conf. Ser. 49: Galaxy Evolution. The Milky Way Perspective, ed. S. R. Majewski, 37-+

Reid, N., Hawley, S. L., \& Mateo, M. 1995b, MNRAS, 272, 828

Reid, N., \& Majewski, S. R. 1993, ApJ, 409, 635

Richards, G. T., et al. 2002, AJ, 123, 2945

—. 2006, AJ, 131, 2766

Robin, A. C., Reylé, C., Derrière, S., \& Picaud, S. 2003, A\&A, 409, 523

Rocha-Pinto, H. J., Flynn, C., Scalo, J., Hänninen, J., Maciel, W. J., \& Hensler, G. 2004, A\&A, 423, 517

Romano, D., Chiappini, C., Matteucci, F., \& Tosi, M. 2005, A\&A, 430, 491

Rubin, V. C., \& Ford, W. K. J. 1970, ApJ, 159, 379

Sako, M., et al. 2005, ArXiv Astrophysics e-prints

Salpeter, E. E. 1955, ApJ, 121, 161

Sandage, A. R., \& Eggen, O. J. 1959, MNRAS, 119, 278

Scalo, J. M. 1986, Fundamentals of Cosmic Physics, 11, 1

Schlegel, D. J., Finkbeiner, D. P., \& Davis, M. 1998, ApJ, 500, 525

Schmidt, M. 1959, ApJ, 129, 243

-. 1963, ApJ, 137, 758

-. 1968, ApJ, 151, 393

Schmidt, S. J., Cruz, K. L., Bongiorno, B. J., Liebert, J., \& Reid, I. N. 2007, AJ, 133, 2258

Schultheis, M., Robin, A. C., Reylé, C., McCracken, H. J., Bertin, E., Mellier, Y., \& Le Fèvre, O. 2006, A\&A, 447, 185

Sesar, B., et al. 2007, ArXiv e-prints, 704 
-. 2008, AJ, in preparation

Shkolnik, E., Liu, M. C., Reid, I. N., Hebb, L., Cameron, A. C., Torres, C. A., \& Wilson, D. M. 2008, ArXiv e-prints, 805

Siegel, M. H., Majewski, S. R., Reid, I. N., \& Thompson, I. B. 2002, ApJ, 578, 151

Silvestri, N. M., et al. 2006, AJ, 131, 1674

Skrutskie, M. F., et al. 2006, AJ, 131, 1163

Smith, J. A., et al. 2002, AJ, 123, 2121

Smolčić, V. et al. 2004, ApJL, 615, L141

Stauffer, J. R., Balachandran, S. C., Krishnamurthi, A., Pinsonneault, M., Terndrup, D. M., \& Stern, R. A. 1997, ApJ, 475, 604

Stauffer, J. R., Liebert, J., Giampapa, M., Macintosh, B., Reid, N., \& Hamilton, D. 1994, AJ, 108, 160

Stobie, R. S., Ishida, K., \& Peacock, J. A. 1989, MNRAS, 238, 709

Stoughton, C., et al. 2002, AJ, 123, 485

Strauss, M. A., et al. 1999, ApJL, 522, L61

-. 2002, AJ, 124, 1810

Terndrup, D. M., Stauffer, J. R., Pinsonneault, M. H., Sills, A., Yuan, Y., Jones, B. F., Fischer, D., \& Krishnamurthi, A. 2000, AJ, 119, 1303

Tinney, C. G. 1993, ApJ, 414, 279

Tonry, J., \& Davis, M. 1979, AJ, 84, 1511

Trumpler, R. J., \& Weaver, H. F. 1953, Statistical astronomy (Dover Books on Astronomy and Space Topics, New York: Dover Publications, -c1953)

Tsvetanov, Z. I., et al. 2000, ApJL, 531, L61

Tucker, D. L., et al. 2006, Astronomische Nachrichten, 327, 821

Vallenari, A., Pasetto, S., Bertelli, G., Chiosi, C., Spagna, A., \& Lattanzi, M. 2006, A\&A, 451, 125

van Altena, W. F., Lee, J. T., \& Hoffleit, E. D. 1995, The general catalogue of 
trigonometric [stellar] paralaxes (New Haven, CT: Yale University Observatory, - c1995, 4th ed., completely revised and enlarged)

van der Kruit, P. C., \& Searle, L. 1981, A\&A, 95, 105

van Leeuwen, F. 2007, A\&A, 474, 653

van Rhijn, P. J. 1925, Publications of the Kapteyn Astronomical Laboratory Groningen, 38, D1+

—. 1936, Publications of the Kapteyn Astronomical Laboratory Groningen, 47, 1

Vanden Berk, D. E. et al. 2005, AJ, 129, 2047

von Seeliger, H. H. 1898, Abhandlugen der K. Bayer. Akademie d. Wiss. II. Kl., Vol. 19, 564-+

Vrba, F. J., et al. 2004, AJ, 127, 2948

Vyssotsky, A. N. 1956, AJ, 61, 201

Walkowicz, L. M., Hawley, S. L., \& West, A. A. 2004, PASP, 116, 1105

Weis, E. W., \& Upgren, A. R. 1995, AJ, 109, 812

Weistrop, D. 1972, AJ, 77, 849

-. 1976, ApJ, 204, 113

West, A. A., Bochanski, J. J., Hawley, S. L., Cruz, K. L., Covey, K. R., Silvestri, N. M., Reid, I. N., \& Liebert, J. 2006, AJ, 132, 2507

West, A. A., Hawley, S. L., Bochanski, J. J., Covey, K. R., Reid, I. N., Dhital, S., Hilton, E. J., \& Masuda, M. 2008, AJ, 135, 785

West, A. A., Walkowicz, L. M., \& Hawley, S. L. 2005, PASP, 117, 706

West, A. A., et al. 2004, AJ, 128, 426

-. 2007, AJ, in preparation

Wielen, R. 1974, Highlights of Astronomy, 3, 395

-. 1977a, A\&A, 60, 263

-. 1977b, A\&A, 60, 263

Wielen, R., Jahreiss, H., \& Krüger, R. 1983, in IAU Colloq. 76: Nearby Stars and 
the Stellar Luminosity Function, ed. A. G. D. Philip \& A. R. Upgren, 163-170

Williams, C. C., et al. 2002, in Bulletin of the American Astronomical Society, Vol. 34, Bulletin of the American Astronomical Society, 1292-+

Willman, B. et al. 2005, AJ, 129, 2692

Wilson, O., \& Woolley, R. 1970, MNRAS, 148, 463

Woolf, V. M., \& Wallerstein, G. 2006, PASP, 118, 218

Xia, F., Ren, S., \& Fu, Y. 2008, Ap\&SS, 314, 51

Yanny, B. et al. 2003, ApJ, 588, 824

Yanny, B., et al. 2000, ApJ, 540, 825

Yoachim, P., \& Dalcanton, J. J. 2006, AJ, 131, 226

—. 2008, ArXiv e-prints, 804

York, D. G., et al. 2000, AJ, 120, 1579

Yoshii, Y. 1982, PASJ, 34, 365

Zheng, Z., Flynn, C., Gould, A., Bahcall, J. N., \& Salim, S. 2001, ApJ, 555, 393

Zwicky, F. 1937, ApJ, 86, 217 


\section{VITA}

John Joseph Bochanski, Jr. was born August 5th, 1980, in Turnersville, New Jersey and grew up in nearby Washington Township. At age 13, sparked by an article in the local paper on the Milky Way's central black hole, he began reading any astronomy books available at the library. This led him to major in the subject at Villanova University, outside Philadelphia. While at Villanova, he worked on solartype stars with Edward Guinan, and developed a passion for studying the stars. After graduating from Villanova in 2002, he headed west, to the University of Washington. During his six years at UW, he has worked closely with Suzanne Hawley, tackling low-mass dwarfs and the local Milky Way, while trying to develop new techniques that take advantage of survey data. He defended and graduated in June 2008. John is moving back east in the fall, heading to Cambridge, MA to work with Adam Burgasser (and be re-united with Andrew West) at MIT. While living in Seattle, John caught the running bug, and has completed one marathon, two halfs, and many other (thankfully) shorter races. He was also a member of the Infrared Sox, anchoring left field for multiple championship teams. Finally, John has never forgotten his roots, emphatically representing South Jersey whenever possible, whether rooting for his beloved Phillies, discussing the relative merits of Springsteen and Bon Jovi, or complaining about the Eagles. 